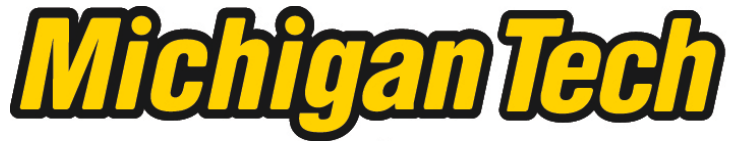 \\ Michigan Technological University Create the Future Digital Commons @ Michigan Tech
}

2013

\section{Modern Computational Chemistry Methods for Prediction of Ground- and Excited-State Properties in Open-Shell Systems}

Nina Tyminska

Michigan Technological University

Follow this and additional works at: https://digitalcommons.mtu.edu/etds

Part of the Chemistry Commons

Copyright 2013 Nina Tyminska

\section{Recommended Citation}

Tyminska, Nina, "Modern Computational Chemistry Methods for Prediction of Ground- and Excited-State Properties in Open-Shell Systems", Dissertation, Michigan Technological University, 2013.

https://doi.org/10.37099/mtu.dc.etds/580

Follow this and additional works at: https://digitalcommons.mtu.edu/etds

Part of the Chemistry Commons 
MODERN COMPUTATIONAL CHEMISTRY METHODS FOR PREDICTION OF GROUND- AND EXCITED-STATE PROPERTIES IN OPEN-SHELL SYSTEMS

\author{
By \\ Nina Tymińska \\ A DISSERTATION \\ Submitted in partial fulfillment of the requirements for the degree of \\ DOCTOR OF PHILOSOPHY \\ In Chemistry
}

MICHIGAN TECHNOLOGICAL UNIVERSITY

2013

@ 2013 Nina Tymińska 
This dissertation has been approved in partial fulfillment of the requirements for the Degree of DOCTOR OF PHILOSOPHY in Chemistry.

Department of Chemistry

Dissertation Co-advisor: Dr. M. Włoch.

Dissertation Co-advisor: Dr. L. Valenzano.

Committee Member: $\quad$ Dr. B. C. Cornilsen.

Committee Member: $\quad$ Dr. L. R. Mazzoleni.

Committee Member: $\quad$ Dr. R. Pati.

Department Chair: Dr. C. F. Chabalowski. 
To Mom, my role model and best friend 


\section{Contents}

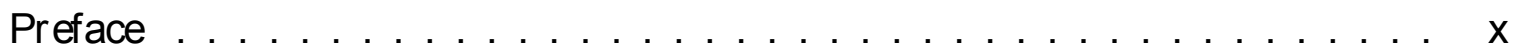

Acknowledgments ..................... . . . . . . .

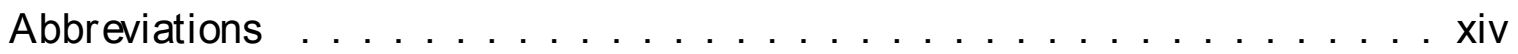

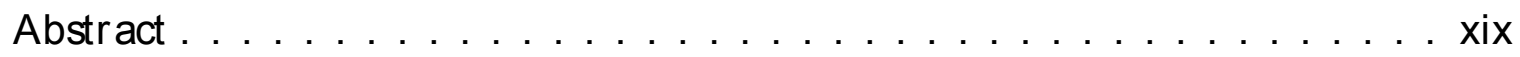

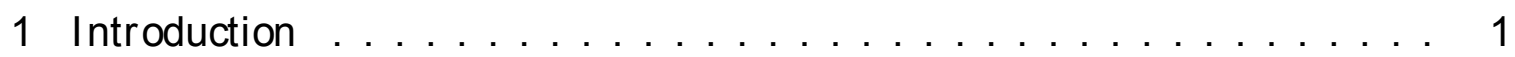

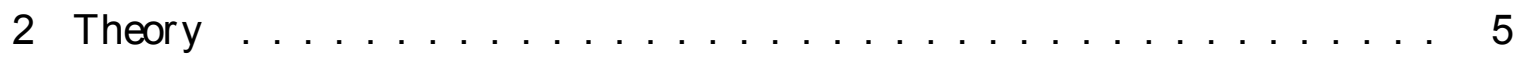

2.1 Brief Overview of Computational Methods . . . . . . . . . . . 5

2.1.1 Self Consistent Field Hartree-Fock . . . . . . . . . . . . . . 6

2.1 .2 Post Hartree-Fock Methods . . . . . . . . . . . . . . . . . . 9

2.1.2.1 Möller-Plesset Perturbation Theory . . . . . . . . . . 9

2.1.2.2 Configuration Interaction . . . . . . . . . . 11

2.1.2.3 Coupled Cluster . . . . . . . . . . . . . . . . 13

2.1 .3 Density Functional Theory . . . . . . . . . . . . . . . . . . 18

2.1.3.1 Time-Dependent DFT . . . . . . . . . . . . . . . 26

2.2 Modern Coupled Cluster Methods and Density Functionals . . . . . . . . 29

2.2.1 Essential s of the Biorthogonal MMCC Theory . . . . . . . . . . 33

2.2.2 Beyond Standard Exchange-Correlation Functionals . . . . . . 42

2.2.2.1 Long-Range Corrected DFT . . . . . . . . . . . . . 43 
3 The Size Extensivity Problem in Excited-State EOM CC M ethods ${ }^{1} \ldots \ldots$

3.1 Brief Introduction . . . . . . . . . . . . . . . . 47

3.2 Motivation and Methodology $\ldots \ldots \ldots \ldots$

3.3 Results and Discussion . . . . . . . . . . . . . . . . . . . . . . . 51

3.4 Insights into the Role of ${ }_{\mu}$ in the Size Extensivity Error . . . . . . . . . 59

3.5 Conclusions . . . . . . . . . . . . . . . . . . . 62

4 UV-Vis Spectra of Copper (II) Complexes with I midazole Derivatives from Long-Range Cor rected Density Functionals ${ }^{2} \ldots \ldots$. . . . . . . . . 63

4.1 DFT and TDDFT Results . . . . . . . . . . . . . . . . . . . . . . . . 69

4.1.1 Structural Parameters . . . . . . . . . . . . . . . . . . . 69

4.1 .2 UV-Vis Spectra . . . . . . . . . . . . . . . . . . . . . 78

4.2 LRC-TDDFT Results and Discussion . . . . . . . . . . . . . . . . . . 89

4.2.1 The Effect of the Range-Separation Parameter and the Fraction of Exact Exchange on Electronic Transitions . . . . . . . . . . . . . 94

4.2.1.1 The Range-Separation Parameter . . . . . . . . . . . 95

4.2.2 MoreInsightsAbout the Performance of the" Tuned" CAM-B3LYP*:

Description Beyond the HOMO/LUMO Energy Levels . . . . . . . 109

4.2.3 $\left[\mathrm{Cu}(\mathrm{IAC})_{2}\right]:$ Results and Discussion $\ldots \ldots \ldots \ldots \ldots$

4.2.3.1 Calculations in Vacuum . . . . . . . . . . . . . . 113

4.2.3.2 Calculations in Solvent . . . . . . . . . . . . . . . . . 124

4.3 Conclusions . . . . . . . . . . . . . . . . . . . . . . . . . 137

5 Influence of Basis Set for Predicting Acidities of First-Row Hydrides and their Lithiated Analogs ${ }^{3} \ldots$. . . . . . . . . . . . . . . . . . . . . . 139

5.1 Introduction . . . . . . . . . . . . . . . . . . . . . . . . . . 139

${ }^{1}$ The material contained in this Chapter was prepared in collaboration with N. Bauman. ${ }^{113}$

${ }^{2}$ Part of the material contained in this Chapter has been adapted from accepted for publication article. ${ }^{120}$ Another part will be used for publication in collaboration with Prof. Bryant (see Preface). ${ }^{121}$

${ }^{3}$ The material contained in this Chapter will be submitted to Int. J. Quantum Chem. $(2013)^{165}$ 
5.2 Method . . . . . . . . . . . . . . . . . . . . . . . . . . . 142

5.3 Results and Discussion . . . . . . . . . . . . . . . . . . . . . . 144

5.4 Triplet Ground State of $\mathrm{LiO}^{-} \ldots \ldots \ldots$

5.5 Conclusions . . . . . . . . . . . . . . . . . . . . 156

6 Concluding Remarks . . . . . . . . . . . . . . . . . . . . 158

References . . . . . . . . . . . . . . . . . . . . . . . . . . 162

\section{List of Figures}

3.1 Hypothetical Morse curves for the ground- and excited-state . . . . . . 61

4.1 Molecular structure of the $\mathrm{Cu}(\mathrm{IA})_{2}$ complex $\ldots \ldots \ldots \ldots . \ldots 70$

4.2 Molecular structure of the $\mathrm{Cu}(\mathrm{IAC})_{2}$ complex $\ldots \ldots \ldots \ldots .70$

4.3 TDDFT UV-Vis spectra cal culated for the $\mathrm{Cu}(\mathrm{IA})_{2}$ complex . . . . . . . 81

4.4 TD-B3LYP UV-Vis spectra cal cul ated for the $\mathrm{Cu}(\mathrm{IAC})_{2}$ complex . . . . . 85

4.5 UV-Vis spectra of the $\mathrm{Cu}(\mathrm{IA})_{2}$ complex in vacuum cal culated with $\mathrm{B} 3 \mathrm{LY}$, CAM-B3LYP, and CAM-B3LYP* . . . . . . . . . . . . . . . . 90

4.6 Lowest excited states of the $\mathrm{Cu}(\mathrm{IA})_{2}$ complex. Influence of the $\mathrm{LR}$ and asymptotic correction on $\beta$-spin d-d transition from HOMO-14 to LUMO . 91

4.7 Lowest excited states of the $\mathrm{Cu}(\mathrm{IA})_{2}$ complex. Influence of the $\mathrm{LR}$ and asymptotic correction on $\beta$-spin $d-d$ transition from HOMO-10 to LUMO . 92

4.8 Comparison of the LRC-TDDFT absorption spectra of the $\mathrm{Cu}(\mathrm{IA})_{2}$ complex in vacuum $\ldots \ldots \ldots$. . . . . . . . . . . . . . . 96

4.9 Comparison of the absorption spectra of the $\mathrm{Cu}(\mathrm{IA})_{2}$ complex $\ldots \ldots .107$

4.10 Trend for sel ected $\alpha$-spin Molecular Orbitals of the $\mathrm{Cu}(\mathrm{IA})_{2}$ complex $\ldots 111$

4.11 Trend for selected $\beta$-spin Molecular Orbitals of the $\mathrm{Cu}(\mathrm{IA})_{2}$ complex $\ldots 112$ 
4.12 Comparison of the absorption spectra of the $\mathrm{Cu}(\mathrm{IAC})_{2}$ complex . . . . . . 120

4.13 Comparison of the LRC-TDDFT absorption spectra of the $\mathrm{Cu}(\mathrm{IAC})_{2}$ complex in vacuum . . . . . . . . . . . . . . . . . . 123

4.14 Solvation effect of methanol and dichloromethane on the shape of dominating MO pair representing $d_{z^{2}} \rightarrow d_{x^{2}-y^{2}}$ transition . . . . . . . 127

4.15 Comparison of the LRC-TDDFT absorption spectra of the $\mathrm{Cu}(\mathrm{IAC})_{2}$ complex in methanol and dichloromethane calculated with CAM-B3LYP* $(\gamma=0.25)$, $\omega B 97 X$ and LC-wPBE . . . . . . . . . . . . . . . . . . 133

4.16 Comparison of the LRC-TDDFT absorption spectra of the $\mathrm{Cu}(\mathrm{IAC})_{2}$ complex in vacuum, methanol and dichloromethane cal culated with CAM$B 3 L Y P^{*}(Y=0.25)$ and CAM-B3LYP* . . . . . . . . . . . . . . 134

5.1 Acidity of first-row hydrides $\left(\mathrm{H}_{n} \mathrm{R}\right)$ and their lithiated anal ogs $\left(\mathrm{LiH}_{\mathrm{n}-1} \mathrm{R}\right)$ vs. electronegativity of $\mathrm{R}$ at $298 \mathrm{~K} \ldots \ldots \ldots \ldots$

5.2 Optimized structures of $\mathrm{LiH}_{\mathrm{n}-2} \mathrm{R}^{-}$molecules $\ldots \ldots \ldots \ldots$

5.3 Optimized structures of $\mathrm{LiH}_{n-1} \mathrm{R}$ molecules $\ldots \ldots \ldots \ldots$

\section{List of Tables}

3.1 Determination of accuracy of the CR-EOMCC, H-CR-EOMCC and EOM-

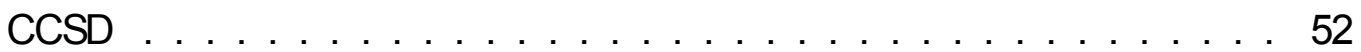

3.2 Size extesivity errors for the vertical excitation energies . . . . . . . . 53

3.3 Excited-state energies and errors with respect to $\mathrm{FCl}$ val ues . . . . . . . 56

3.4 Vertical excitation energy values for $\mathrm{CH}^{+}, \mathrm{H}_{2} \mathrm{CO}$, and $\mathrm{N}_{2} \ldots \ldots \ldots$

3.5 Vertical excitation energy values for $\mathrm{H}_{2} \mathrm{O}$ and $\mathrm{C}_{2} \ldots \ldots \ldots \ldots$

4.1 DFT/LL2DZ cal culated structural parameters of the $\mathrm{Cu}(\mathrm{IA})_{2}$ complex $\ldots 71$ 
4.2 DFT/SRSC calculated structural parameters of the $\mathrm{Cu}(\mathrm{IA})_{2}$ complex $\ldots 72$

4.3 DFT/CCDPP cal cul ated structural parameters of the $\mathrm{Cu}(\mathrm{IA})_{2}$ complex $\ldots 73$

4.4 Structural parameters of the $\mathrm{Cu}(\mathrm{IA})_{2}$ complex obtained with B3LYP/CCDPP with respect to different basis sets for ligand atoms $\ldots \ldots \ldots \ldots$

4.5 Structural parameters of the $\mathrm{Cu}(\mathrm{IAC})_{2}$ complex $\ldots \ldots \ldots \ldots . .77$

4.6 Selected electronic transitions and oscillator strengths for the $\mathrm{Cu}(\mathrm{IA})_{2}$ complex from TDDFT cal culations . . . . . . . . . . . . . . . . . . . 79

4.7 TDDFT predicted d-d transitions for the $\mathrm{Cu}(\mathrm{IAC})_{2}$ complex . . . . . . 83

4.8 BP86 predicted LMCT transitions with corresponding oscillator strength for the $\mathrm{Cu}(\mathrm{IAC})_{2}$ complex . . . . . . . . . . . . . . . . 86

4.9 B3LYP predicted LMCT transitions with corresponding oscillator strength for the $\mathrm{Cu}(\mathrm{IAC})_{2}$ complex . . . . . . . . . . . . . . . . . . . 87

4.10 M06L predicted LMCT transitions with corresponding oscillator strength for the $\mathrm{Cu}(\mathrm{IAC})_{2}$ complex . . . . . . . . . . . . . . . . . . 88

4.11 LRC-TDDFT predicted $d-d$ transitions for the $\mathrm{Cu}(\mathrm{IA})_{2}$ complex in vacuum . 97

4.12 Percentage of Cu-d and the $\beta$-spin orbital energies obtained from population analysis for given $\mathrm{LRC}$ functionals for the $\mathrm{Cu}(\mathrm{IA})_{2}$ complex $\ldots \ldots .99$

4.13 Cal culated vertical IP(N) of the neutral $\mathrm{Cu}(\mathrm{IA})_{2}$, SCF-DFT energies of neutral complex and its cation, orbital energies of $\mathrm{HOMO}(\mathrm{N})$, and $\mathrm{J}^{2}(\mathrm{Y})$ values 104

4.14 CAM-B3LYP* predicted LMCT transitions with corresponding oscillator strength, and $\mathrm{S}^{2}$ for the $\mathrm{Cu}(\mathrm{IA})_{2}$ complex in vacuum . . . . . . . . 108

4.15 Calculated vertical IP(N) of the neutral $\mathrm{Cu}(\mathrm{IA})_{2}$ complex, SCF-DFT energies of neutral complex and its cation, orbital energies of $\mathrm{HOMO}(\mathrm{N})$ and $\mathrm{J}^{2}(\mathrm{y})$ values . . . . . . . . . . . . . . . . . . . . . . . . . 109

4.16 LRC-TDDFT predicted d-d transitions for the $\mathrm{Cu}(\mathrm{IAC})_{2}$ complex in vacuum 115

4.17 Percentage of $\mathrm{Cu}-\mathrm{d}$ and the $\beta$-spin orbital energies obtained from the population analysis for a given $\mathrm{LRC}$ functional for the $\mathrm{Cu}(\mathrm{IAC})_{2}$ complex $\ldots 117$ 
4.18 CAM-B3LYP* LMCT transitions with corresponding oscillator strength, and $\mathrm{S}^{2}$ for the $\mathrm{Cu}(\mathrm{IAC})_{2}$ complex in vacuum . . . . . . . . . . 121

4.19 LRC-TDDFT predicted LMCT transitions with corresponding oscillator strength, and $\mathrm{S}^{2}$ for the $\mathrm{Cu}(\mathrm{IAC})_{2}$ complex in vacuum . . . . . . . . 122

4.20 Percentage of $\mathrm{Cu}-\mathrm{d}$ and $\beta$-spin orbital energies obtained from population analysis with given $\mathrm{LRC}$ functional for the $\mathrm{Cu}(\mathrm{IAC})_{2}$ complex in vacuum, $\mathrm{CH}_{3} \mathrm{OH}$, and $\mathrm{CH}_{2} \mathrm{Cl}_{2} \ldots \ldots \ldots \ldots \ldots . \ldots \ldots$

4.21 LRC-TDDFT predicted d-d transitionsfor the $\mathrm{Cu}(\mathrm{IAC})_{2}$ complex in methanol and dichlorometane . . . . . . . . . . . . . . . . . . . . . . . . . . . . . 129

4.22 LRC-TDDFT predicted LMCT transitions with corresponding oscillator strength and $S^{2}$ for the $\mathrm{Cu}(\mathrm{IAC})_{2}$ complex in methanol and dichloromethane 130

5.1 Cal culated Energies and Acidities in kcal/mol using Dunning's basis sets at OK . . . . . . . . . . . . . . . . . . . . . . . . . 146

5.2 Cal culated Zero Point Energies and Acidities in $\mathrm{kcal} / \mathrm{mol}$ using Dunning's basis sets at 298K . . . . . . . . . . . . . . . . . . . . . . . . . 148

5.3 Cal culated Energies and Acidities in $\mathrm{kcal} / \mathrm{mol}$ using Pople's basis sets at OK 149

5.4 Cal culated Zero Point Energies and Acidities in kcal/mol using Pople's basis sets at $298 \mathrm{~K} \ldots \ldots \ldots$

5.5 Comparison between CCSD and CCSD(T) results in predicting acidities . . 153 


\section{Preface}

This dissertation is submitted in partial fulfillment of the requirements for a Degree of Doctor of Philosophy $(\mathrm{PhD})$ in Chemistry. It contains work done during 5 years of the Chemistry PhD Program at Michigan Technological University.

For the first four and one-half years my advisor on projects was Dr. Marta Wloch. For the last two semesters (Spring 2013 and Summer 2013) Dr. Loredana Valenzano became my co-advisor. In addition, during 16 months (Fall 2012- Fall 2013) of the Cooperative Education Program at Oakland University in Rochester, MI, my on campus co-advisor was Dr. Bahne C. Cornilsen. The dissertation has been prepared solely by the author, however corrections and changes to the original text were made with the help of Dr. Wloch and Dr. Val enzano.

The research presented in this dissertation has been done by the author as well as in collaboration others: (i) Mr. Nicolas Bauman - computational laboratory undergraduate student of Dr. Wloch (Chapter 3), (ii) Dr. Ferman Chavez and Dr. Maria M. Szczesśniak (Bryant) (Chapter 4), and (iii) Dr. Wloch and Dr. Tim Royappa (Chapter 5).

The role of the collaborators in each of the described chapters is listed below.

Mr. Nicolas Bauman prepared a fragment of a loop code that was specifically designed to test the extensive properties of the Equation-of-Motion Coupled Cluster methods. The author served as supervisor and helped in debugging the code.

Dr. Ferman Chavez provided the X-ray data for the $\mathrm{Cu}(\mathrm{II})$ complex with bis(2-(1-ethyl$1 \mathrm{H}$-imidazol-4-yl)acetate copper(II) and his experimental expertise in the area of inorganic chemistry. This was helpful in writing results and discussion about the UV-Vis spectra 
modeling performed independently by the author.

Dr. Maria M. Szczesśniak (Bryant) was involved in advising the part of the research about the effects of the range-separation parameter and exact exchange on UV-Vis spectra of $\mathrm{Cu}(\mathrm{II})$ with 4-imidazole acetic acid. In addition Dr. Bryant provided computational time and access to software packages which allowed the author to efficiently perform the required cal culations. The author proposed the main idea for this part of the research as well as performed all the cal cul ations necessary to obtain the results presented herein.

Dr. Royappa introduced to the author and Dr. Wloch the key idea leading to the project by stressing the importance of the basis set in predicting the acidity of first-row hydrides and their lithiated anal ogs. The author performed all the cal cul ations as well as participated in writing the article.

The author has done her best to provide references to the sources of the material used in her PhD Dissertation. 


\section{Acknowledgments}

It would not be possible to write this dissertation without the help and support of some truly great people I had the opportunity to work and share my doctoral experience with. It would take a foot long list if I could mention all of them, hence I hope they understand that herein I only mention some particular individuals.

This dissertation would not exist without the help, support and patience of my advisor, Dr. Marta Wloch. Her advices and expertise in the area of theoretical methods in chemistry were invaluable during my $\mathrm{PhD}$ program. I truly appreciate her giving me a chance to prove myself, and doing it with friendliness and kind nature.

In addition, the presented work would not be in the shape it is now if not for the great advice, assistance in writing, and never ending enthusiasm of my co-advisor, Dr. Loredana Val enzano. Also, her friendship has been priceless in my academic and personal life. I am very grateful for her involvement in both.

I would like to acknowledge the financial, academic and technical support of Michigan Technological University. In particular, I want to thank the Chemistry Department for the Graduate Teaching Assistant scholarship which provided the necessary financial support for me, without which I would not be able to accomplish the research presented in this work. Furthermore, for similar reasons, I would like to mention the Chemistry Department of Oakland University at Rochester, where I spent 16 months during the Cooperative Educational Program.

I am most grateful to Prof. Maria M. Szczesniak (Bryant) for providing advise and computer resources during my Co-op experience. Her exceeding knowledge in the area of Density Functional Theory was especially val uable for part of the research I present in this 
work.

Another OU faculty I would like to acknowledge is Prof. Ferman Chavez, without whom I wouldn't have got involved in the research on $\mathrm{Cu}(\mathrm{II})$ complexes. This project turned out to be most rewarding. I also appreciate him sharing with me his experimental expertise in inorganic chemistry which was helpful in writing the discussion and conclusions for this part of my dissertation.

Among graduate students in OU I truly appreciate the efforts of Mrs. Jessica V. Koppen in providing not only a stimulating and welcoming academic environment but also offering me her friendship and help whenever I needed it.

Last, but by no means least, I thank my family, and my friends among fellow graduate students at MTU: Rosa Flores, Nazmiye Yapici, Xi Lin (Sissi), and Evandro Ficanha for their friendship and encouragement during all the years I spent in Houghton, MI.

I take the full responsibility of any errors and/or inadequacies that may be contained in this dissertation. 


\section{Abbreviations}

ACCD: Augmented Correlation-Consistent polarized valence-only Double-zeta basis set ACCT: Aug-CC-pv Triple-zeta basis set

ALDA: Adiabatic Local Density Approximation

B.O.: Born-Oppenheimer

B3LYP: Becke's three parameter hybrid functional combined with Le, Yang, and Par correlation functional

BCC: Brueckner Coupled Cluster

$\mathrm{BD}(\mathrm{T})$ : Brueckner Doubles with non-iterative Triples

BP86: Becke's exchange functional combined with Perdew 86 correlation functional

CAM-B3LYP* : Asymptotical ly corrected CAM-B3LYP

CAM-B3LYP: Long-range corrected version of B3LYP using the Coulomb-Attenuating Method

CAS-AQCC: Complete Active Space Averaged Quadratic Coupled Cluster

CC: Coupled Cluster

CC3: Linear response functions for approximate CC triples models

CCD: CC Doublets

CCDPP: cc-pVDZ Plus Polarization effective core potential

CCDZ: Correlation-Consistent polarized val ence-only Double-Zeta basis set

CC-LRT: CC Linear Response Theory

$\operatorname{CCSD}(2)_{\mathrm{T}}$ : Hirata's CCSD plus non-iterative perturbative Triples

CCSD: CC Singles, and Doubles

$\operatorname{CCSD}(T)$ : CCSD plus non-iteratively added perturbative Triples

CCSD[T]: Alternative approach for adding non-iterative perturbative Triples

CCSDT: CC Singles, Doubles, and Triples

CCSDTQ: CCSD plus Triples, and Quadruples

CCTZ: CC-pv Triple-Zeta basis set 


\section{$\mathrm{Cl}$ : Configuration Interaction \\ CID: CI Doublets}

CISD: $\mathrm{Cl}$ approach including Singly and Doubly excited configurations

CISDTQ: CISD plus Triply and Quadruply excited configurations

CR-CC $(2,3)$ : Biorthogonal variant of CR-CCSD with included non-iterative perturbative Triples a.k.a. CR-CCSD $(T)_{L}$

CR-CC: Completely-Renormalized CC

$\mathrm{CR}-\mathrm{CC}_{\mathrm{L}}$ : Biorthogonal CR-CC

CR-CCSD(T): Completely-Renormal ized CCSD $(T)$

CR-CCSD(TQ): Completely-Renormal ized CCSD(TQ)

CR-EOM CC $(2,3), A$ : Variant of CR-EOMCC $(2,3)$ with Möller-Plesset type of perturbative denominator

CR-EOMCC(2,3),D: Variant of CR-EOMCC(2,3) with Epstein-Nesbet type of perturbative denominator

CR-EOM CCSD (2,3): Biorthogonal variant of Completely-Renormalized EOMCCSD with included non-iterative perturbative Triples a.k.a. $\operatorname{CR}-\operatorname{EOMCCSD}(T)_{L}$

CR-EOM CCSD(T),ID/IA: ID/IA mixed variant of CR-EOMCCSD(T), i.e., the excitation energy is obtained by subtracting the CR-EOMCCSD(T),IA ground-state energy from the CR-EOMCCSD(T),ID energy of excited state

CR-EOM CCSD(T),ID/IB: ID/IB mixed variant of CR-EOMCCSD(T), i.e., the excitation energy is obtained by subtracting the CR-EOMCCSD $(T)$,IB ground-state energy from the CR-EOMCCSD(T),ID energy of excited state

CR-EOM CCSD(T),ID: ID variant of CR-EOMCCSD(T) use perturbative expressions for approximate wave functions in terms of cluster components $\left(T_{1}, T_{2}\right)$ and excitation components $\left(R_{0}, R_{1}, R_{2}\right)$ with full treatment of perturbative denominator CR-EOM CCSD(T): Completely-Renormalized EOMCCSD plus non-iteratively added perturbative Triples

CSF: Configuration State Function 
CT: Charge Transfer

cx: Carboxylate

DFA: Density Functional Approximation

DFT: Density Functional Theory

EA: Electron Affinity

EA-EOM CC: Electron Attachment EOMCC

ECP: Effective Core Potential

EE: Excitation Energy

EE-EOM CC: Excitation Energy EOMCC

EOM CC: Equation-of-Motion Coupled-Cluster

EOM CCSD(T): EOMCCSD plus non-iteratively added perturbative Triples

EOM CCSD: EOMCC Singles, and Doubles

EOM-CCSDT-1: EOMCCSD with iterative treatment of Triples

$\mathrm{FCl}$ : Full Configuration Interaction

GABA: Gamma Aminobutyric Acid

GGA: Generalized Gradient Approximation

H-CR-EOM CC(2,3),A: Modified CR-EOMCC(2,3),A using Hirata's idea to remove size-

extensivity error

H-CR-EOM CC(2,3),D: Modified CR-EOMCC(2,3),D using Hirata's idea to remove size-

extensivity error

HF: Hartre-Fock

HOMO: Highest Occupied Molecular Orbital

IA: Imidazole-4 acetate

IAC: 1-Ethyl-1H-Imidazol-4-yl acetate

IEF-PCM: Integral Equation Formalism of The Polarizable Continuum Model

im: Imidazole

IP: Ionization Potential

IP-EOM CC: Ionization Potential EOMCC 
KS: Kohn-Sham

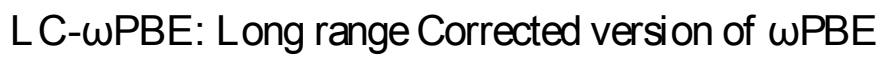

LDA: Local Density Approximation

LF: Ligand Field

LL2DZ: Los Alamos National Laboratory Double-Zeta ECP

LLCT: Ligand to Ligand CT

LMCT: Ligand to Metal CT

LR: Long-Range

LRC: Long-Range Corrected

LRC-TDDFT Long-Range Corrected TDDFT

LSDA: Local-Spin-Density Approximation

LUMO: Lowest Unoccupied Molecular Orbital

M06L: Minnesota 06 family Local functional

mACCD Modified ACCD basis set

MAE: Maximum Absolute Error

MBPT: Many Body Perturbation Theory

mGGA: Meta GGA

MMCC: Methods of Moments of Coupled Cluster

$M_{M C C}$ : Biorthogonal MMCC formalism

MO: Molecular Orbital

MP: Möller-Plesset

MRCC: Multireference Coupled Cluster

OE: Orbital Energy

PA: Proton Affinity

PBE: Perdew, Burke, and Ernzerhof functional

PES: Potential Energy Surface

PUMP:Projection Unrestricted MP

QCISD: Quadratic CISD 
R-CC: Renormalized CC

$\mathrm{R}-\operatorname{CCSD}(\mathrm{T})$ : Renormilzed $\operatorname{CCSD}(\mathrm{T})$

R-CCSD(TQ): Renormilzed CCSD with included non-iterative perturbative Triples and Quadruples

REL: Relative Excitation Levels

RG: Runge-Gross theorem

RHF: Restricted HF

ROHF: Restricted Open-shell HF

RPA: Random Phase Approximation

RSPT: Rayleigh-Schrödinger Perturbation Theory

SAC-Cl: Spin-Adapted Cluster Configuration Interaction

SCF: Self-Consistent Field

SCF-HF: SCF Hartree-Fock

SF-CISD: Spin-Flip CISD

SIE: Self-Interaction Error

SOM O: Singly Occupied MO

SRCC: Single-Reference CC

SR: Short-Range

SRSC: Stuttgart Relativistic Small Core 1997 ECP

TD: Time-Dependent

TDDFT: Time-Dependent DFT

UHF: Unrestricted HF

UMP: Unrestricted MP

UV-Vis: UltravioletVisible

VWN: Vosko, Wilk, and Nusair correlation functional

wB97X: LRC Becke's 97 hybrid functional

$x c$ : Exchange-Correlation

ZPE: Zero-Point vibrational Energy 


\section{Abstract}

The main goal of the research presented in this work is to provide some important insights about computational modeling of open-shell species. Such projects are: the investigation of the size extensivity error in Equation-of-Motion Coupled Cluster methods, the anal ysis of the Long-Range corrected scheme in predicting UV-Vis spectra of $\mathrm{Cu}(\mathrm{II})$ complexes with the 4-imidazole acetate and its ethylated derivative, and the exploration of the importance of choosing a proper basis set for the description of systems such as the lithium monoxide anion. The most significant findings of this research are: (i) The contribution of the left operator to the size extensivity error of the CR-EOMCC $(2,3)$ approach, (ii) The cause of $d$ $d$ shifts when varying the range-separation parameter and the amount of the exact exchange arising from the imbal anced treatment of local ized vs. delocal ized orbitals via the "tuned" CAM-B3LYP* functional, (iii) The proper acidity trend of the first-row hydrides and their lithiated anal ogs that may be reversed if the basis sets are not correctly sel ected. 


\section{Chapter 1}

\section{Introduction}

The aim of the research presented in this dissertation is to provide some val uable insights into theoretical/computational modeling of open-shell species, i.e., molecules with unpaired electron(s). The process of bond breaking, occurring during chemical reactions, often involves radicals or even bi radicals. Usually these species are short lived, and highly reactive intermediates or transition states. Hence, it is difficult to determine their properties experimental ly; this makes electronic structure theory a val uable if not necessary tool of investigation. Without the ability to accurately and reliably describe open-shell species many chemical reaction mechanisms would not be completely understood. This is the reason why studies such as the one presented in this work are essential in many disciplines such as: atmospheric chemistry, material science, biochemistry, synthesis of metal-organic complexes, and photo-chemistry. Additionally, open-shell systems are interesting to study from a theoretical point of view. For example, the lone electron may lead to changes in chemical behavior of a species (Chapter 5), the characterization of their excited states is more demanding (Chapter 3), and the description of spectroscopic properties is even far more difficult (and Chapter 4). 
In this work a few problems emerging from the use of computational methodologies in describing properties of open-shell systems are addressed. These include some major chal lenges encountered by electronic structure theory in modeling the UV-Vis spectra of metal-organic complexes. This is because highly accurate ab initio methods based on the wave-function approach are too costly in terms of computational effort. Even with relatively small system, where highly accurate ab initio methods such as Coupled Cluster can be employed the so-called size extensivity error, when truncated approximations of such methods are applied to exited states, can occur. An additional problem is the finite basis set approximation that may lead not only to large errors in values for the properties but also to qual itatively wrong predictions. Therefore, the main goal of this work is to provide more insights into these three problems applying modern theoretical methods to open-shell systems.

Chapter 2 ("Theory") provides basic concepts, equations and formal ism necessary to understand the modern computational methods employed in this work. Among these Coupled Cluster and Density Functional Theory play a major role in studies presented herein; essential approximations of these models will be described in detail focusing on recently devel oped approaches.

Chapter 3 ("The Size Expressivity Problem in Excited State EOMCC") address vertical excitation and excited state energies of various systems through Coupled Cluster calculations. The size extensivity properties of the Completely-Renormalized approach based on Biorthogonal Methods of Moments of Coupled-Cluster equations (the so-called CREOMCC $(2,3)$ ) method are examined. The main reason behind this study was to find possible source of the size-extensivity error arising from this scheme. Furthermore, research presented in this chapter was inspired by the fact that the ground-state $\mathrm{CR}-\mathrm{CC}(2,3)$ is free of the size-extensivity error. Size extensivity is an important requirement for theoretical methods, because the proper description of some important processes such as bond break- 
ing is assured for such approaches.

Chapter 4 ("UV-Vis Spectra of Copper(II) Complexes with Imidazole Derivatives from Long-Range Corrected Density Functionals") is dedicated to the spectroscopic properties of open-shell metal-organic complexes. This part employs theoretical methodologies based on the electron density (Density Functional Theory), which constitutes an al ternative way of obtaining electronic structures and properties of systems of interest. In particular, Time Dependant Density Functional Theory using several Long-Range Corrected exchange-correlation functionals was examined to determine the performance of this relatively recent development in predicting the absorption spectra of copper(II) complex with 4-imidazole acetate and 1-ethyl-1H-imidazol-4-ylacetate. The main motivation was twofold:

† Investigate the ability of the Long-Range Corrected scheme for exchange-correlation functional s to describe both metal- and ligand- based electronic transitions with similar accuracy for the challenging systems considered herein,

† Explore the possible reasons for shifts occurring in the $d-d$ transitions positions. Our results suggest that these are affected by both the range separation parameter $(\mathrm{\gamma})$ and fraction of Hartree-Fock exchange ( $\alpha$ ). The latter investigation was mainly preformed using CAM-B3LYP* functional and can be helpful in understanding the effect of these parameters on electronic transitions involving d-electron energy levels.

To the best of our knowledge such studies were not presented in the literature, hence they provide a great novelty.

Chapter 5 ("Influence of Basis Set for Predicting Acidities of First-row Hydrides and their Lithiated Anal ogs" ) discusses the importance of the choice of the basis set. The key idea of such investigation was to show that the so-called "black-box" type methods (for example, 
$\operatorname{CCSD}(T)$ ) may be utilized to obtain chemical properties such as acidity/basicity of species with unusual multiplicity for the ground state species, i.e., different than singlet, if only the basis set is properly chosen. Our results prove that the augmented basis sets are necessary for the appropriate predictions of acidities of first-row hydrides and, more importantly their lithiated anal ogs, which due to the involvement of $\mathrm{Li}$ atom exert some peculiar properties. Our calculations show that the correct acidity order is achieved by augmenting relatively small cc-pVXZ $(X=D, T)$ basis sets. A similar effect is observed for the family of Pople's basis sets. 


\section{Chapter 2}

\section{Theory}

\subsection{Brief Overview of Computational Methods}

The use of computers in chemistry can be dated at the beginning of 50's where the first softwares became available, however few publications predate that date in the history of computational chemistry. The fist papers on quantum mechanics by Heisenberg ${ }^{1}$ and Shrödinger ${ }^{2}$ as well as Hückel's work on $\pi$ electrons theory ${ }^{3}$ were published in the late 20 's. With the progressing development of theoretical methods, and the improvement of computer codes the interest in employing computational models for solving various chemical problems increased. Numerous text books and review articles reporting the progress of theoretical and/or computational chemistry are available, therefore only a brief overview of the most commonly utilized methods will be presented herein. Note that since the Coupled Cluster and Density Functional Theory have been applied in studies presented later in this text the approximationsinherent in these models will be described in greater detail focusing on recently developed approaches. Also, this section can be considered as a historical introduction since it provides the foundations necessary to understand modern computational 
methods.

\subsubsection{Self Consistent Field Hartree-Fock}

The Schrödinger equation cannot be solved for many-body systems, therefore employing approximated methods such as the Self-Consistent Field Hartree-Fock (SCF-HF) is necessary in order to obtain solutions which are cl ose to the "true" solution. In this method the variational principle is applied with a single Slater determinant, in the so-cal led mean field, to obtain approximated ground-state wave functions and their eigenvalues (i.e., energies), iteratively.

In case of the electronic Schrödiger equation, the mean field arises from all the other electrons in the system with respect to a single electron, considered to be independent. It is well known that this and other approximations, made to simplify the many-electron system problem (for which an exact solution is not known), cause substantial shortcomings in the description of real systems. The most significant error in SCF-HF method arose from neglecting of electron correlation. Further errors are considered to be consequences of the clamped nuclei Born-Oppenheimer (B.O.) approximation, neglecting of relativistic effects, the use of finite Gaussian basis set, and the description of wave functions by single Slater determinant.

The single-determinant approximation is the biggest limitation, as it represents a nonphysical description for the many-electron system. This approximation is especially insufficient for systems where two or more orbital s are nearly or completely degenerate. The energy corresponding to this effect is called "non-dynamical correlation" energy. ${ }^{4}$ Efforts in including both dynamical and non-dynamical electron correlation energies led to the development of so-called post-Hartree-Fock methods, such as Möller-Plesset Perturbation 
Theory, Configuration Interaction and Coupled Cluster, described later in this work.

The Hartree-Fock differential equations have a form ${ }^{5}$

$$
F(1) \varphi_{i}(1)=\varepsilon_{i} \varphi_{i}(1)
$$

where, $\varphi_{i}$ is a i ${ }^{\text {th }}$ spin-orbital, $\varepsilon_{i}$ its corresponding orbital energy. The Fock operator $(F)$ is defined as follows

$$
F(1) \equiv-\frac{1}{2} \nabla_{1}^{2}-\sum_{\alpha} \frac{Z_{\alpha}}{r_{1 \alpha}}+\sum_{j=1}\left[J_{j}(1)-K_{j}(1)\right]
$$

where, $\mathrm{J}_{\mathrm{j}}$ is the Coulomb operator (electron-electron repulsion) and $\mathrm{K}_{\mathrm{j}}$ is the exchange operator that has no classical equivalent. The spatial part of the Hartree-Fock orbitals ( $\varphi=X c$, where $X$ is the basis function and $c$ is its coefficient) can be represented as a linear combination of known functions; for example, Gaussian atomic-orbital functions (called basis functions) can be used. The complete set of coefficients specifying the normalized contributions of the basis functions to the molecular orbitals is found iteratively during the SCF procedure. The HF wave function can be found via the Roothaan equation ${ }^{5}$

$$
\mathrm{FC}=\mathrm{SC} \varepsilon
$$

where, $S=S_{i j}=\left\langle X_{i} \mid X_{j}\right\rangle$ is the overlap matrix, $C$ is the vector containing the coefficients, and $\varepsilon$ is the diagonal square matrix, whose el ements $\varepsilon_{i}$ are the orbital energies. As mentioned before, the SCF-HF method utilized Slater determinant define bel ow

$$
\begin{aligned}
& \varphi_{1}(1) \quad \varphi_{2}(1) \quad \ldots \quad \varphi_{N}(1)
\end{aligned}
$$

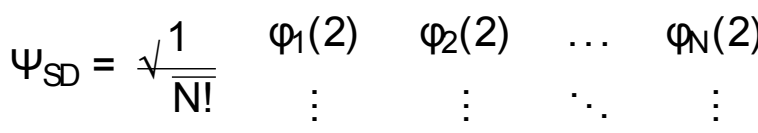

$$
\begin{aligned}
& \varphi_{1}(\mathrm{~N}) \quad \varphi_{2}(\mathrm{~N}) \quad \ldots \quad \varphi_{\mathbb{N}}(\mathrm{N})
\end{aligned}
$$

In general, the time-independent Schrödinger equation is given as

$$
H \Psi=E \Psi
$$


where the Hamiltonian operator $\mathrm{H}$ is given by the sum of kinetic and potential energy operators ${ }^{6}$

$$
H=T_{n}+T_{e}+V_{n e}+V_{e}+V_{n n}
$$

In Eq. (2.6) the first two terms are the nuclei and electronic kinetic energy operators, and the remaining three terms represent nucleus-electron, electron-electron and nucleus-nucleus potential energy operators, respectively.

Due to B.O. approximation, the Hamiltonian operator can be reduced to the electronic Hamiltonian $\left(\mathrm{H}_{\mathrm{e}}\right)$, which defined in atomic units has the form

$$
H_{e}=-\sum_{i=1}^{N} \frac{1}{2} \nabla^{2}\left(r_{i}\right)-\sum_{i}^{N} \sum_{\alpha} \frac{Z_{\alpha}}{r_{i \alpha}}+\sum_{i}^{N} \sum_{j>1}^{N} \frac{1}{r_{i j}}+\sum_{\alpha} \sum_{\beta>\alpha} \frac{1}{r_{\alpha \beta}}
$$

where, $r_{i}$ defines the position of the $i$-th electron, $r_{\alpha}$ is the position of the $\alpha$-th nucleus, and $Z_{\alpha}$ is the atomic number of nucleus $\alpha$. The last term of in Eq. (2.7) is the nuclear potential repul sion energy, and it is a constant.

It is necessary to mention that SCF-HF method can be applied to both closed-shell (i.e., singlet type system with paired electrons on all the orbitals) and open-shell systems. The Restricted Hartree-Fock (RHF) method is used for closed-shell systems; all previous and further discussion refers to this model, unl ess stated differently. In case of systems with unpaired el ectrons, cal culations can be handl ed with either Restricted Open-shell HF (ROHF) or Unrestricted HF (UHF); the former does not al low for different spatial orbital functions of electrons which are paired with each other, additional ly ROHF functions are eigenfunctions of the $S^{2}$ operator, which represents the squared total electron spin. The UHF does not restrict paired electrons, i.e., their spatial orbital functions are different and therefore can correctly describe dissociation. On the other hand, its wave function is not an eigenvalue of $S^{2}$; this may cause errors called spin contamination (i.e., unreal mixing of states with different multiplicity; singlets with odd-higher lying states and doublets with even-higher lying states). ${ }^{7}$ 


\subsubsection{Post Hartree-Fock Methods}

For most atomic, molecular and solid state calculations the HF method is applied as the starting point. For this reason it is important to introduce basic concepts behind the postHartree-Fock methods. First, ab initio methods for ground state cal culations based on the single determinant such as Möller-Plesset perturbation theory, Configuration Interaction, and Coupled Cluster will be shortly discussed. Next, their extensions to excited states will be addressed.

\subsubsection{Möller-Plesset Perturbation Theory}

Möller-Plesset (MP) perturbation theory ${ }^{8}$ al so known as Many Body Perturbation Theory (MBPT) is one of the major ab initio post-HF methods: it attempts to retrieve the electroncorrelation energy. This method is based on Rayleigh-Schrödinger Perturbation Theory (RSPT),${ }^{5}$ which expands the reference wave function (i.e., the unperturbed HF wave function) and its corresponding energies as a Taylor's series of the perturbation parameter $\lambda$, $(0<\mathrm{n}<1)$. The RSPT that takes into account all the allowed excitations truncated according to the given order is indicated as MBPT, and it is size-extensive. This property is essential for chemical applications, because it al lows to obtain meaningful properties such as heat of reaction, activation energy, an accurate relative energies al ong the Potential Energy Surface (PES), as well as between different electronic states.

MPn, where $\mathrm{n}$ is the order of corrections included in the power series, consider the unperturbed Hamiltonian $\left(\mathrm{H}^{0}\right)$ as equal to the sum of the one-electron Fock operators

$$
H^{0}=\sum_{i=1}^{N} F_{i}
$$


where, the superscript describe the correction-order, $\mathrm{N}$ is the number of electrons in the system, and $F_{i}$ is defined in Eq. (2.2). The MPn equations may be written as

$$
\left(H^{0}+\lambda H\right)\left|\Psi_{0}\right\rangle=a\left|\Psi_{0}\right\rangle
$$

where, the subscript 0 denotes the ground state and the perturbation $\mathrm{H}$ can be written as

$$
H=H-H^{0}=\sum_{i} \sum_{i>j} \frac{1}{r_{i j}}-\sum_{i=1}^{N} \sum_{j=1}^{N}\left[J_{i j}-K_{i j}\right]
$$

The perturbation changes continuously, therefore we can write Eq. (2.9) as a Taylor series expansion of $\lambda$ (i.e., unitless parameter that when varied from 0 to 1 maps $\mathrm{H}^{(0)}$ to $\mathrm{H}$ )

$$
\begin{gathered}
\left(H^{0}+\lambda H\right)\left|\Psi^{0}+\lambda \psi^{1}+\lambda^{2} \Psi^{2}+\ldots\right\rangle= \\
\left(a^{0}+\lambda a^{1}+\lambda a^{2}+\ldots\right)\left|\psi^{0}+\lambda \psi^{1}+\lambda^{2} \Psi^{2}+\ldots\right\rangle
\end{gathered}
$$

where $\Psi^{1}=\frac{\partial \psi^{0}}{\partial \lambda}, \Psi^{2}=\frac{\partial \psi^{0}}{\partial \lambda^{2}}$, etc.

The operator H (Eq. (2.10)) is applied to incorporate perturbation theory in order to correct $\mathrm{HF}$ reference wave function. The first order MP does not improve energy beyond HF level, because $E^{1}=\left\langle\Psi^{0}|H| \Psi^{0}\right\rangle$, and since $\Psi^{0}=\Phi^{H F}$, we have that $E^{0}+E^{1}=\left\langle\Phi\left|H^{0}+H\right| \Phi\right\rangle$, which yields to HF energy. Hence, to improve the HF energy the lowest possible level of MP method has to be greater than one, i.e., MP2, MP3, etc.

The most commonly up level used is the second order (MP2). Higher orders rarely exceed MP4, which is next most common. The reason behind this is that interaction effects between paired electrons dominate in the Hamiltonian, despite other interactions might be important as well (see discussion in sections 2.1.2.2 and 2.1.2.3). MP2 is a very efficient method, as its computational cost is $\mathrm{N}^{5}$, where the scaling behavior is described as a function of the number of basis functions $\mathrm{N}$. Additionally, this method offers analytical gradients and Hessians, which all ow exploring PESs. Yet, because MPn is not a variational method, the correlation energy may be wrongly estimated, and the total energy can be 
lower than the "exact energy" of the system (see section 2.1.2.2). Another issue is the unpredictable convergence behavior of higher orders, i.e., slow, erratic, oscillatory, depending on the problem and/or basis set (see, for example, Leininger et al. ${ }^{9}$ ). Also, MPn methods are not applicable to excited states, which may appear as a disadvantage in comparison to the other post-HF methods. Nevertheless, MP2 with its desirable cost to accuracy ratio is the most economical post-HF method, and it continues to be developed. For example, MP2-R12 ${ }^{10}$ and MP2-F12 ${ }^{11}$ are MP2 "flavours" developed with the aim to minimizeerrors arising in cal culating finite basis sets (Levine, ${ }^{12}$ section 16.2). Finally, there is no reason to not utilize UHF and ROHF wave functions for open-shell system cal culations, with UMPn

and ROHF MPn methods, respectively. Note that unlike for UHF, the projection method ${ }^{13}$ aimed to eliminate the spin contamination is quite efficient for UMPn. Unfortunately, since PUMP involves twice as many molecular orbital coefficients and given the cost of projection itself, its computational cost is twice that of MP.

\subsubsection{Configuration Interaction}

Full Configuration Interaction ( $\mathrm{FCl}$ ) method gives the best possible solution (or exact solution) to the time-independent Schrödinger equation within a given basis set, where typical assumptions are kept (i.e, non-relativistic, B.O. approximated). This method considers the contribution of every possible Configuration State Function (CSF), where each CSF is a linear combination of Slater determinants $\sum_{i} c_{i} \Phi_{i}$, and $c_{i}$ are coefficients defining the weight of a given configuration. The CSFsare classified according to the number of excited electrons as singly, doubly, triply, ..., n-tuply excited states (configurations), with one, two, thre, ..., n-electrons excited from occupied to virtual orbitals, respectively.

The FCl wave function can be written as follows

$$
\Psi=c_{0} \Psi_{H F}+\sum_{i}^{o c c} \sum_{a}^{\text {vir. }} c_{i}^{a} \Psi_{i}^{a}+\sum_{i<j}^{o c c} \sum_{a b}^{\text {vir. }} c_{i j}^{a b} \Psi_{i j}^{a b}+\ldots
$$


It is not hard to real ize that with growing number of electrons and size of basis set calculations become more and more complex and demanding due to the huge amount of configurations; in fact, the number of determinants grows factorially. This limits the applicability of $\mathrm{FCl}$ method to all but small systems, i.e, small molecules (few light atoms) with relatively small basis set. Therefore, a truncation of the configuration interaction expansion is needed to lower the cost of the cal culations. However this aspect goes together with loos in accuracy. For example, $\mathrm{Cl}$ approach including singly and doubly excited configurations (CISD) recovers between $82-90 \%$ of the correlation energy for molecules built from atoms of the first row, but it decreases drastical ly with increasing number of electrons; for a 100electrons problem recovery of the correlation energy drops to only $55-67 \% .{ }^{12}$ In order to obtain more accurate results with truncated $\mathrm{Cl}$ triply and quadruply excited configurations (CISDTQ) needs to be included, but again the cost of such calculations is nearly as prohibitive as that of $\mathrm{FCl}$. Another problem arises from the fact that truncated $\mathrm{Cl}$ is neither size extensive nor size consistent, where the former term refers to energy scaling properly (i.e., linearly) with the size of the system and it applies to all geometries, and the letter term applies only to infinitely separated parts whose sums must be equal to the energy of the system as a unity $\left(E_{A B}=E_{A}+E_{B}\right)$. Size-consistency is extremely important. In fact, methods lacking of this property cannot describe properly dissociation. This implies that every method based on RHF is not size-consistent, while being size-extensive. ${ }^{4}$ The LanghoffDavidson ${ }^{14}$ method (often abbreviated as QCISD) is one of more popular methods to deal with the size-consistency problem. The QCISD formula is given below

$$
\Delta \mathrm{E}_{\mathrm{Q}} \approx\left(1-\mathrm{a}_{0}^{2}\right)\left(\mathrm{E}_{\mathrm{CISD}}-\mathrm{E}_{\mathrm{SCF}}\right)
$$

where $\mathrm{a}_{0}$ is the coefficient of the HF wave function in the normal ized CISD expansion, and $\mathrm{E}_{\mathrm{CISD}}$ and $\mathrm{E}_{\mathrm{SCF}}$ are the $\mathrm{CISD}$ and HF energies, respectively. Eq. (2.13) takes into account the so-called unlinked quadruples, i.e., quadruply excited configurations built from simultaneously excited doubles. Note that a more recent version of the CISD method is able to account for size-consistency and it is variational; this is the Spin-Flip CISD (SF-CISD) developed by Krylov, ${ }^{15}$ which is more robust than other non-spin-flip approaches and it 
deal s better with homolytic bond breaking energy and biradical singlet-triplet separation. ${ }^{16}$

Why determinants higher than doubly-excited are important? Doubles (or doubly excited determinants) give the largest contribution in terms of energy; this can be proven by calculating their weight (i.e., the sum of the $\mathrm{c}_{i}^{2}$ coefficients) at a given level of $\mathrm{Cl}$ method. The next most important determinants would be singles because they make the wave function more flexible by allowing orbitals to "relax". After singles, there are quadruples and triples, which al so are quite significant; the former can be seen as a product of two doubly excited determinants counting for electron pairs interactions, the latter contribute to the multi-reference wave function built in the $\mathrm{Cl}$ method. This means that since triply excited determinants can be treated as doubly excited with respect to singles, the system is now approximately multi-referenced. Since both Triplets and Quadruplets can be expressed in terms of Doublets, which are the most important, by not including them relatively significant amount of correlation energy may be missing leading to some undesirable effects such as lack of size-extensivity (for details see discussion in section 2.1.2.3). Contributions from determinants with higher then four excited electrons are usually very small. ${ }^{6}$

\subsubsection{Coupled Cluster}

The foundations of Coupled Cluster (CC) theory ${ }^{17-24}$ have been established from attempts of solving the nuclear many-body problem in the mid-fifties. This elegant method would probably be forgotten by nuclear physicists if it was not for the chemist Jiri Čižek. In his works published in 1966, Čižžk ${ }^{19}$ presented the explicit coupled-cluster equations for electrons in the language of quantum chemistry. Later (1972), Paldus ${ }^{21}$ applied the CC equations to solve chemical problems. More than a decade after Čižek's first work the first multipurpose program capable of applying CC method became available. ${ }^{22}$ The coupled cluster singles and doubles, cal led CCSD ${ }^{25}$ developed and utilized by Purvis and Bartlett 
in 1982, became one of the most common CC approaches. While CCSD reproduces the correct curvature of the potential energy with respect to the bond length (the so-called Morse's potential curve), the energy of the system in its geometrical equilibrium is too high comparing to results from $\mathrm{FCl}$. In order to lower the energy and obtain the correct potential energy curve, higher than doubly excited determinants have to be considered. Unfortunately increasing the level of CC theory substantial ly increases the computational cost of the calculations. For CCSD this cost scales as $N^{6}$ or $n_{\text {occ }}^{4} n_{\text {vir }}^{2}\left(n_{\text {occ }} n_{\text {vir }}\right.$ are the number of occupied/unoccupied or virtual orbitals, and $\mathrm{N}$ is a general measure of the system size); for $\operatorname{CCSDT}^{26,27}$ (i.e., CCSD with iteratively included full triples) it becomes $\mathrm{N}^{8}$ $\left(\mathrm{n}_{\mathrm{occ}}^{3} \mathrm{n}_{\mathrm{vir}}^{5}\right)$; CCSDTQ scales as $\mathrm{N}^{10}\left(\mathrm{n}_{\mathrm{occ}}^{4} \mathrm{n}_{\text {vir }}^{6}\right)$, and its cost is prohibitively high for most but the smallest systems. Therefore, the development of less expensive CC approaches able to give results with an accuracy close to that of $\mathrm{FCl}$, is still to be achieved. Note that sice CC includes all the possible excitations, its energy is "exact" within a given basis set, as it is for the FCl method. Since 1989 the CC approach with singles, doubles and non-iteratively added perturbative triples, called $\operatorname{CCSD}(T)^{28}$ (labelled as the " gold standard of quantum chemistry"), has been the most popular choice among chemists, due to its desirable accuracy-to-cost ratio. However, it is well known that this method cannot be used for systems involving degenerate states such as molecules far from their geometrical equilibrium, and for systems involving large contribution of the " non-dynamical" correlation energy (see section 2.1.1). Note that the computational cost of non-iterative triples in $\operatorname{CCSD}(T)$ approach is $N^{7}\left(n_{\text {occ }}^{3} n_{\text {vir }}^{4}\right)$, which is not only cheaper than full CCSDT, but it is also better balanced than its precursor from 1985 CCSD[T], proposed by Urban et al. ${ }^{29}$ More details about these two and other " standard" single-reference approximated $\mathrm{CC}$ method can be found, for example, in the review by Bartlett and Musiat. ${ }^{30}$

This overview would not be complete without mentioning that CC theory can be easily implemented to obtain excited states via the Equation-of-Motion Coupled-Cluster (EOM-CC) method. This method was first introduced by Emrich ${ }^{31,32}$ in 1981 and applied to mol ecular 
systems using its singles and doubles extension (EOM-CCSD) by Comeau and Bartlett ${ }^{33}$ in 1993. While EOM-CC methods al low to some extent to deal with many multireference states, they may require to employ multi-determinant reference in CC theory. This can be achieved by applying Multireference Coupled Cluster (MRCC) methods, which became popular within the computational-chemistry community thanks to most recent advances. ${ }^{34}$

In CC theory the cluster operator $\mathrm{T}$, acts on the reference wave function $\Phi_{0}$ to create $\mathrm{n}$-tuple cluster functions, where $\mathrm{n}$ is the number of correlated electrons. This can be done by representing the exponential cluster operator $\mathrm{e}^{\top}$ as an expansion of cluster operator $\mathrm{T}$ representing the cluster expansion wave function

$$
\mathrm{e}^{\mathrm{T}} \equiv 1+\mathrm{T}+\frac{\mathrm{T}^{2}}{2 !}+\frac{\mathrm{T}^{3}}{3 !}+\cdots=\sum_{\mathrm{k}=0}^{\infty} \frac{\mathrm{T}^{\mathrm{k}}}{\mathrm{k} !}
$$

The cluster operator $\mathrm{T}$ in the Eq. (2.14) is defined as

$$
\mathrm{T} \equiv \mathrm{T}_{1}+\mathrm{T}_{2}+\ldots+\mathrm{T}_{\mathrm{n}}
$$

where, $T_{1} \Phi_{0} \equiv \sum_{a=n+1}^{\infty} \sum_{i=1}^{n} t_{i}^{a} \Phi_{i}^{a}, T_{2} \Phi_{0} \equiv \sum_{b=a+1}^{\infty} \sum_{a=n+1}^{\infty} \sum_{i=1}^{n} \sum_{j=i+1}^{n-1} t_{i j}^{a b} \Phi_{i j}^{a b}$, etc.

Thus, the fundamental $\mathrm{CC}$ equation is

$$
\Psi=\mathrm{e}^{\top} \Phi_{0}
$$

and it is solved for the coefficients $t_{i}^{a}, t_{j i}^{a b}, \ldots$ (amplitudes), for all the $i, j, \ldots$ occupied orbitals and for all the $a, b, \ldots$ virtual orbital s. If the cluster operator includes all possible $T_{n}$, the obtained wave function would be exact as in the case of $\mathrm{FCl}$.

What novelty does CC theory bring to electronic structure theory? The answer lays in approximated CC approaches, where the cluster operator is truncated. The simplest way to demonstrate the superiority of truncated CC methods comes from examining of CID (Eq. (2.17)) and CCD (Eq. (2.18)) wave functions. The latter contains higher than secondorder excitations, which the most important contribution is the term $\frac{1}{2} \mathrm{~T}_{2}^{2}$; this term approximates quadruple excitations, and gives more significant contribution to the correlation 
energy than the energy arising from simultaneously excited four electrons, i.e., $T_{4}$. This approach same cannot be applied to truncated $\mathrm{Cl}$ until this method reaches the CISDQ approximation, because it treats each excited determinant independently:

$$
\begin{gathered}
\psi^{C I D}=1+C_{0} \Phi_{0}+C_{2} \Phi_{i j}^{a b} \\
\psi^{C C D}=e^{T_{2}} \Phi_{0}=\left(1+T_{2}+\frac{1}{2} T_{2}^{2}\right) \Phi_{0}
\end{gathered}
$$

The additional term in CCD created by the product of $T_{2}$, is the reason behind its sizeextensivity: in general the exponential form of the $T$ operator ensures size-extensivity of truncated CC approaches. It has to be emphasized that this property is essential for chemical application of computational methods in prediction of meaningful properties such as heat of formation, activation energies, relative energies along PES as well as between different electronic states.

In fact, CCD is the lowest CC level beyond HF. However, this method is not commonly used because CCSD is a more complete approach and the computational cost of adding singles is negligible (i.e, both CCD and CCSD scale with the system size $(N)$ as $N^{6}$ ). The next level adds triples, but CC with full triples (CCSDT), is al ready too demanding for all but small systems, as its cost scales as $\mathrm{N}^{8}$. In summary, there are several methods able to approximate the effect of $\mathrm{T}_{3}$, being $\operatorname{CCSD}(\mathrm{T})$ the most wildly used. In $\operatorname{CCSD}(\mathrm{T})$ where the perturbative triples are included noniteratively, due to their relatively low cost (CCSD $N^{6}+(T) N^{7}$ ) high accuracy for nondegenerated ground state molecular systems can be obtained in an easy to use fashion (so-called "black-box" type method). However, chemists are often not only interested in obtaining accurate results for molecules in their geometrical equilibrium but also when the bond breaking can occur (i.e., chemical reaction), where the perturbative correction leads to a failure of the $\operatorname{CCSD}(T)$ method. This is due to fact that the energy difference between two Slater determinants translates into a difference in molecular orbitals energies. Such difference decreases when the bond is stretched until it is degenerated causing the energy of the $\mathrm{SD}(\mathrm{T})$ correction to drop to minus infinity. The iterative inclusion of approximated effects of triples is also available via 
CCSDT-1 and CCSDT-3, ${ }^{35}$ the difference between these two methods is that the latter includes all the possible contributions coming from $T_{2}$ and $T_{1}$ into $T_{3}$, where additional terms with respect to the CCSD model are the si milarity-transformed Hamiltonian $\left(\bar{H}=e^{-\top} H^{\top}\right)$ intermediates. Both methods avoid storage of $t_{i j k}^{a b c}$ amplitudes and save one order $(\sim N)$ in scaling behavior when compared to full CCSDT ${ }^{30}$ Non-iterative treatment of truncated $\mathrm{T}_{3}$ in CCSDT-1, leads to the method called CCSD[T], which after some modification is more rigoristic in terms of size-extensivity $\operatorname{CCSD}(\mathrm{T})$ approach. Quadruples and higher order excitations can be similarly approximated (iteratively and non-iteratively), avoiding the scaling behavior of the full method; for example, the most complete approximated approach of the full CCSDTQ $\left(\mathrm{N}^{10}\right)$ is $\operatorname{CCSDT}(\mathrm{Q})\left(\mathrm{N}^{9}\right)^{36}$ Therefore, further improvements of $\mathrm{CC}$ methods need modifications able to minimize the error arising from the single-determinant reference rather than the inclusion of higher order excited states. CC methods which include these improvements are called Modern Coupled Cluster methods, and will be further discussed (Section 2.2).

A final aspect needs to be addressed: the choice of the single determinant reference and associated orbitals, which in case of CC theory is completely arbitrary. This choice can be made between determinants such as RHF, ROHF, UHF, Kohn-Sham, ${ }^{37}$ Natural ${ }^{38}$ Brueckner. ${ }^{39}$ The first one is the most popular choice, and among the non-HF determinants the last is commonly used. ${ }^{40}$ When Brueckner orbitals are used in Slater determinant, the contribution from singles is zero due to the orthogonal ity of singly excited state with exact wave function $\left(\left\langle\Phi_{i}^{a} \mid \Psi\right\rangle\right.$ for $0 \leq \mathrm{i} \leq \mathrm{M} \leq \mathrm{a}$, where $\mathrm{M}$ is a number of particles) and the Brueckner wave function $\left(\Phi_{\mathrm{B}}\right)$ corresponding to the exact wave function $(\Psi)$ is such that $\left\|\Psi-\Phi_{\mathrm{B}}\right\|=\min$ for $\|\Psi\|=\left\|\Phi_{\mathrm{B}}\right\|=1$, i.e., the Brueckner determinant has the maximum overlap with exact wave function CC methods based on this idea are abbreviated BCC. ${ }^{30,40}$ 


\subsubsection{Density Functional Theory}

Density Functional Theory (DFT) is an electronic-structure method utilized for the prediction of crystalline, molecular, and atomic properties, where the goal is to extract all the information from the density (vs. the wave function) of a nondegenerate ground state system. Today DFT is one of the most commonly used tools employed in theoretical chemistry due to its lower computational cost than, for example, Coupled Cluster (CC) methods, for systems with hundreds or more atoms. This was not always the case; before the mideighties DFT was mainly applicable to solid states, where simple approaches based on the uniform electron gas model usually worked quite well. Chemical problems needed more sophisticated approximations. ${ }^{41}$

It is well-known that the explicit mathematical expression for the density functional energy which would define the relationship between the density of the system and its ground state energy remains elusive. ${ }^{42,43}$ Although, the Hohenberg-Kohn theorem ${ }^{44}$ proved that such functional exists and it can befound by minimizing density functional. ${ }^{4}$ Nevertheless, the biggest challenge of DFT, to the day, is to determine the approximation of the "unknown" energy density functional. Furthermore, the performance of DFT methods highly depends on the choice of functional within a given chemical problem; this makes it even more important for a user of computational chemistry packages to be aware of advantages and pitfalls of chosen functionals. Consequently, it is no surprise that many studies have been devoted to the classification of the performance of DFT functionals, for example, see work of Sousa et al. ${ }^{45}$

The Kohn-Sham (KS) DFT approach ${ }^{37}$ is the most popular among others, (e.g., Legendre transformation from the chemical potential to the electron density of $\mathrm{N}$ number of parti-

$\left.\mathrm{cles}^{46}\right)$. Within this framework the arduous many-body problem of electrons interacting in a fixed external potential is simplified to a manageable problem of non-interacting el ectrons 
moving in an effective potential which includes the exchange and correlation Coulomb interactions between the electrons and the interaction with an external potential. Unlike the Hartree-Fock (HF) wave function based theory, the KS DFT methodology is exact conceptually. However the form of one term of the exchange-correlation functional, $\left(E_{x c}\right)$, is unknown. This is a paramount problem as the $E_{x c}$ functional is a key ingredient necessary to solve KS DFT equations. Therefore, finding the analytical form which would approximate this functional is the holy grail of DFT. The number of new functional s increases at an accelerating pace as a result of searching for better and better $E_{x c}$ approximation. ${ }^{43}$

The theoretical background on density functionals will be briefly presented herein to familiarize the reader with commonly used terms and give necessary fundamentals about this method.

DFT uses the ground state electron density instead of the wave function in order to find a ground state energy of a system, by minimizing the energy functional $(E[\rho])$. Hohnberg and Kohn first proved that the so-called external potential can be uniquely determined by the ground state electron density give the Hamiltonian operator, which then is employed to solve Schrödinger equation (this is known as the first Hohnberg-Kohn theorem ${ }^{37,44}$ ). A second theorem states that any guessed density of the system will provide an energy that is greater or equal to the exact energy; this step is achieved via the variational principle. Although these theorems assure that the ground state energy corresponds to the minimum of $E[\rho]$, they do not provide an indication on how to obtain the energy functional itself. ${ }^{47}$

The general form of $E[\rho]$ contains the kinetic energy $(T[\rho])$, the external nuclear-electron potential $\left(V_{n e}[\rho]\right)$, the electron-electron interaction potential $\left(V_{e e}[\rho]\right)$, and the nucleus-nucleus repulsion potential $\left(V_{n n}\right)$, and it can be written as

$$
E[\rho]=T[\rho]+V_{n e}[\rho]+V_{e}[\rho]+V_{n n}
$$

where $\rho$ is the ground state el ectron density. 
The exact expressions of $T[\rho]$ and $V_{e}[\rho]$ are unknown. The sum of these two (taken as an average of the electronic Hamiltonian) gives the definition of the exchange-correlation energy functional ${ }^{12}$

$$
\mathrm{E}_{\mathrm{xc}} \equiv \Delta \overline{\mathrm{T}}[\rho]+\Delta \overline{\mathrm{V}}_{e}[\rho]
$$

where $\Delta \overline{\mathbf{T}}$ is the difference in the average electronic kinetic energy between the molecule in its ground state and the reference system (see below). The $\Delta \bar{V}_{e e}$ term is defined as

$$
\Delta \bar{V}_{\circledast} \equiv \bar{V}_{\circledast}-\frac{1}{2} \quad \frac{\rho\left(r_{1}\right) \rho\left(r_{2}\right)}{r_{12}} d r_{1} d_{1} r_{2}
$$

In Eq. (2.21) $\bar{V}_{\circledast}$ is the average non-classical electron-electron repul sion, $r_{12}$ is the distance between the electronic coordinates of electrons 1 and 2, and the double integral represents the classical electrostatic interelectronic repulsion energy (in atomic units) for electrons spread out onto a continuum of charge with $\rho$.

The Kohn-Sham (KS) DFT approach al lows to approximate them, using the known density of non-interacting electrons as a reference system, for which the density coincides to that of the real system. ${ }^{12}$ In KSDFT E[p] is then given as

$$
E[\rho]=T_{s}[\rho]+V_{n e}+J[\rho]+E_{x c}[\rho]+V_{n n}
$$

where $T_{s}[\rho]$ denotes the kinetic energy of the reference system. Note that $T_{s}[\rho]=T[\rho]$, because $T[\rho]$ is the kinetic energy functional of the real system. $J[\rho]$ and $E_{x c}[\rho]$ are the Coulomb (electron-electron repulsion) energy and introduced earlier exchange-correlation functional, respectively.

The set of KS equations has to be solved in order to obtain the ground state density as

$$
\rho(r)=\sum_{i=1}^{n}\left|\Phi_{i}(r)\right|^{2}
$$

where $r=(x, y, z)$ and $\Phi_{i}$ are the KS one-electron orbitals. 
This is done by minimizing $E[\rho]$ and solving the KS equations

$$
\mathrm{f}^{\mathrm{KS}} \Phi_{\mathrm{i}}=\varepsilon_{\mathrm{i}} \Phi_{\mathrm{i}}
$$

where $\varepsilon_{\mathrm{i}}$ are orbital energies and the $\mathrm{f}^{\mathrm{KS}}$ operator is defined as

$$
f^{K S}(r) \equiv-\frac{1}{2} \nabla^{2}+v_{n e}(r)+j(r)+v_{x c}(r)
$$

The first three terms represent kinetic energy, nuclear-electron interaction, and Coulomb operators, respectively. The $\mathrm{f}^{\mathrm{KS}}$ is the pure electronic single reference Hamiltonian. Equations (2.24) and (2.25) can rewritten as

$$
h_{i} \Phi_{i}\left(r_{1}\right)=\varepsilon_{i} \Phi_{i}\left(r_{1}\right)
$$

and

$$
h_{i} \equiv-\frac{1}{2} \nabla_{1}^{2}-\sum_{A=1}^{N} \frac{Z_{A}}{r_{A i}}+\frac{\rho\left(r_{2}\right)}{r_{12}} d r_{2}+v_{x c}\left(r_{1}\right)
$$

where the exchange-correlation potential $\mathrm{v}_{\mathrm{xc}}$ has to be found, and is defined as

$$
v_{x c}[\rho] \equiv \frac{\delta E_{x c}[\rho(r)]}{\delta \rho(r)}
$$

The expression for the electronic ground state energy for a n-electrons and $\mathrm{N}$-nuclei system can be written as follows

$$
\begin{aligned}
E[\rho]= & -\frac{1}{2} \sum_{i=1}^{n} \Phi_{i}^{*}\left(r_{1}\right) \nabla_{i}^{2} \Phi_{i}\left(r_{1}\right) d r_{1} \\
& -\sum_{A=1}^{N} \frac{Z_{A}}{r_{A i}} \rho\left(r_{1}\right) \\
& +\frac{1}{2} \quad \frac{\rho\left(r_{1}\right) \rho\left(r_{2}\right)}{r_{12}} d_{1} r_{1} d r_{2} \\
& +E_{x c}[\rho]
\end{aligned}
$$

where, the first term accounts for the kinetic energy of noninteracting electrons, the second describes the nuclei-electron interactions, the third represents the Coulomb repul sions of electrons at positions $r_{1}$ and $r_{2}$, and the last is the exchange-correlation energy functional. 
Thus, once $E_{x c}$ is known the density of the system (Eq. (2.23)) and the corresponding energy (Eq. (2.29)) can be found.

The more general treatment of density functionals is provided when electron spin is included; this approach is called spin-DFT ${ }^{48}$ and in the absence of a magnetic field leads to an $E_{x c}$ functional of the type $E_{x c}\left[\rho_{\alpha}(r), \rho_{\beta}(r)\right]$, where $\rho_{\alpha}(r)$ and $\rho_{\beta}(r)$ are the spin $\alpha$ (up) and $\beta$ (down) electron densities.

Despite the $E_{x c}$ appears in the formulation of the KS theory, its unique formula is unknown and therefore the $v_{\mathrm{xc}}$ has to be approximated by finding the exchange-correlation functional able give the best match to the exact form of the exact operator.

Three levels of $E_{x c}$ functionals have been developed: Local Density Approximation (LDA) and its spin-dependant equival ent Local-Spin-Density Approximation (LSDA), Generalized Gradient Approximation (GGA) and meta-GGA (mGGA).

The $E_{x c}$ functional is composed by two parts, exchange $(x)$ and correlation (c); when added together they give the exchange-correl ation functional $\left(E_{x c}[\rho]=E_{x}[\rho]+E_{c}[\rho]\right)$. Both parts of $E_{x c}$ can be treated at the same level of approximation or at different levels.

LDA or LSDA is the least accurate level. Its approximation is based on the uniform electron gas where non-interacting el ectrons move in the space of hypothetical electrically neutral system, filled by a positive charge that is continuously and uniformly distributed. This means that $E_{x c}$ is a function only of $\rho_{\alpha}(r)$ and $\rho_{\beta}(r)$ varying with position $r$. It is important to note that in spin-polarized DFT both electron and spin densities are fundamental quantities with the net spin density being the difference between density of $\alpha$ and $\beta$ electrons $\left(\rho_{\sigma}(r)=\rho_{\alpha}(r)-\rho_{\beta}(r)\right)$ and the total density is a sum of the two.

$$
-\frac{1}{2} \nabla_{1}^{2}-\sum_{A=1}^{N} \frac{Z_{A}}{r_{A i}}+\frac{\rho\left(r_{2}\right)}{r_{12}} d r_{2}+v_{x c}\left(r_{1}\right) \quad \Phi_{i, \sigma}\left(r_{1}\right)=\varepsilon_{i, \sigma} \Phi_{i, \sigma}\left(r_{1}\right)
$$


where $\sigma=\alpha, \beta$.

Furthermore the exchange-correlation functional is usually different for the $\alpha$ and the $\beta$ case, this leading to two sets of spin-polarized Kohn-Sham equations producing $\alpha$ and $\beta$ spin orbitals (Eq. (2.30)). ${ }^{49}$

A typical expression used to represent $E_{x c}$ in LDA is

$$
E_{\mathrm{xC}}^{\mathrm{LDA}}[\rho]=\rho(r) \varepsilon_{\mathrm{xc}}(\rho) \mathrm{d}(\mathrm{r})
$$

were $\varepsilon_{x c}(\rho)=\varepsilon_{x}(\rho)+\varepsilon_{c}(\rho)$ and for which the exchange energy per electron $\left(\varepsilon_{x}\right)$ is given by

$$
\varepsilon_{x}(\rho)=-\frac{3}{4} \quad \frac{3}{\pi}^{1 / 3}(\rho(r))^{1 / 3}
$$

and the correlation energy per electron $\left(\varepsilon_{c}\right)$ is obtained from some known function. ${ }^{12}$ For example, $\varepsilon_{\mathrm{c}}(\rho)=\varepsilon_{\mathrm{C}}^{\mathrm{VWN}}(\rho)$, where $\varepsilon_{\mathrm{C}}^{\mathrm{VWN}}(\rho(r)$ is cal culated via VWN correlation functional. .50

GGA corrects LDA by adding the dependency from the density gradient (i.e., the density change typically indicated as $\nabla \rho$ ) to the dependency from the density at a given point. The general formula for GGA $E_{x c}$ can be written as

$$
E_{\mathrm{xC}}^{\mathrm{GGA}}[\rho]=f(\rho(r) \nabla \rho(r)) d(r)
$$

or including spin densities $\left(\rho_{\alpha}(r), \rho_{\beta}(r)\right)$

$$
E_{\mathrm{xC}}^{\mathrm{GGA}}[\rho]=f\left(\rho_{\sigma}(r), \nabla \rho_{\sigma}(r)\right) d(r) ; \quad \sigma=\alpha, \beta
$$

For instance a popular GGA functional sisBPW91 which involves a combination of Becke's exchange ${ }^{51}$ functional (usually denoted as B or B88), and Perdew and Wang correlation functional .52

The mGGA goes beyond GGA by including the second derivative of the density $\left(\nabla^{2} \rho^{\alpha, \beta}\right)$. 
An al ternative form of the mGGA functional may depend only on the Kohn-Sham kineticenergy density ${ }^{12}\left(T_{\sigma}\right)$ :

$$
T_{\sigma} \equiv \frac{1}{2} \sum_{i=1}^{\text {occupied }}\left|\nabla \Phi_{i \sigma}\right|^{2} ; \quad \sigma=\alpha, \beta
$$

Therefore the general formula for $E_{x c}$ within mGGA is ${ }^{12}$

$$
E_{x C}^{m G G A}[\rho]=\quad f \quad \rho_{\sigma}(r), \nabla \rho_{\sigma}(r), \nabla^{2} \rho_{\sigma}(r), T_{\sigma} \quad d(r) ; \quad \sigma=\alpha, \beta
$$

An example of mGGA functional which gives good results for many properties is TPSS. ${ }^{53}$

Besides the mentioned "pure" DFT functionals, another type is widely used. These are the so-called hybrid functionals, built by hybridizing (or mixing) the $\mathrm{E}_{\mathrm{x}}^{\mathrm{HF}}$, which is defined by HF-exchange formula, with $E_{x}$ and $E_{c}$ GGA or mGGA terms. As an example of hybrid GGA the B3LYP functional will be described in more detail. The version of B3LYP implemented in Gaussian is the Becke's three-parameter functional, ${ }^{54}$ where the correlation functional PW91 ${ }^{52,55}$ is replaced with the correlation functional by Lee-Yang-Parr (LYP). ${ }^{56}$ Consequently, the formula of the unmodified B3LYP is given by the following expression

$$
\begin{aligned}
E_{x C}^{B 3 L Y P}= & a_{0} E_{x}^{H F}+\left(1-a_{0}\right) E_{x}^{L S D A}+a_{x} \Delta E_{x}^{B 88} \\
& +E_{c}^{L S D A(P W 92)}+a_{c} \Delta E_{c}^{L Y P}
\end{aligned}
$$

where

$$
a_{0}=0.20, a_{x}=0.72, a_{c}=0.81
$$

The values of these empirical parameters $\left(a_{0}, a_{x}, a_{c}\right)$ were optimized by fitting series of atomization energies, ionization potentials, proton affinities and atomic energies of systems built from elements of first two rows of the periodic table for B3PW91. ${ }^{54}$ The $\mathrm{E}_{\mathrm{x}}^{\mathrm{HF}}$ is the Hartree-Fock exchange energy, $E_{X}^{L S D A}$ is the Slater's LSDA method (see, Levine ${ }^{12}$ for more details), $\Delta \mathrm{E}_{\mathrm{x}}^{\mathrm{B} 88}$ is the GGA $\mathrm{B} 88$ exchange, ${ }^{51} \mathrm{E}_{\mathrm{C}}^{\mathrm{LSDA}(\mathrm{PW} 92)}$ is Perdew-Wang parameterizations ${ }^{57}$ of LSDA correlation, and $\Delta \mathrm{E}_{\mathrm{C}}^{\mathrm{LYP}}$ is GGA LYP correlation. 
The expression in Eq. (2.37) has to be modified, as the LYP cannot be separated into local and nonlocal correlation expressions, ${ }^{58}$ therefore instead of $E_{C}^{\mathrm{LSDA}(P W 92)}$ the $\left(1-a_{C}\right) E_{C}^{V W N}$ term is used, giving the formula of B3LYP as

$$
\begin{aligned}
E_{x c}^{B 3 L Y P}= & 0.2 E_{x}^{H F}+0.8 E_{x}^{L S D A}+0.72 \Delta E_{x}^{B 88} \\
& +0.19 E_{c}^{V W N}+0.81 \Delta E_{c}^{L Y P}
\end{aligned}
$$

The above equation defines the original expression of B3LYP, although as it can be found in work by Scuseria and Staroverov ${ }^{41}$ that is not the default B3LYP implemented within the Gaussian software package. In their work those explained the differences in details, and so here we only note that the $\mathrm{VWN}^{50}$ term is a key ingredient that alters between B3LYP variants. More precisely, in case of Gaussian the default B3LYP functional has the expression

$$
\begin{aligned}
E_{x c}^{B 3 L Y P}= & 0.2 E_{x}^{H F}+0.8 E_{x}^{L S D A}+0.72 \Delta E_{x}^{B 88}+ \\
& 0.19 E_{c}^{V W N 1-R P A}+0.81 \Delta E_{c}^{L Y P}
\end{aligned}
$$

where RPA is the Random-Phase Approximation to the density functional correlation energy that improves upon nonlocal treatment of electron-electron correlation.

An example of hybrid mGGA functional is TPSSh ${ }^{53}$ which is based on the earlier mentioned TPSS functional.

In summary, many "flavors" of DFT approximations are currently available; according to Grimme ${ }^{59}$ exchange-correlations can be ranked with respect to their accuracy in the following way:

† LDA where exchange-correlation functional depend only on the electron density,

† GGA where both $\rho$ and $\nabla \rho$ are involved,

† mGGA which in addition to $\rho$ and $\nabla \rho$ contain $\nabla^{2} \rho$ or $\mathrm{T}$, 
$†$ Hybrid functionals which mix exact (HF) exchange,

$\dagger$ Double hybrid functionals which add the dependence on the virtual KS orbitals.

\subsubsection{Time-Dependent DFT}

Time-Dependent (TD) DFT can be applied to excited states of atomic or molecul ar systems because electronic excitations as the processes of describing photon-electron interactions are time dependent.

The Runge-Gross (RG) theorem is a time-dependent equival ent of the first Hohnberg-Kohn theorem; it guarantees the existence of a uniquely defined external potential for the initial non-degenerate state $\Psi_{0}$ and the time evolution of electron density the $\rho(r, t)$. The RG theorem defines the so-cal led action integral (A), described by Eq. (2.40), that is interpreted as a functional of the density:

$$
A[\rho]={ }_{t_{0}}^{t_{1}} \Psi[\rho](r, t) i \frac{\partial}{\partial t}-H(r, t) \Psi[\rho](r, t) d t
$$

This is necessary because the total energy of time-dependent systems is not mai ntained and the variational principle cannot be directly applied to find the energy. Nevertheless, the variation of $A[\rho]$ with respect to $\rho(r, t)$ can be found, and the exact density of the system is obtained by solving the time-dependent KS equations

$$
\mathrm{i} \frac{\partial}{\partial \mathrm{t}} \Phi_{\mathrm{i}}(\mathrm{r}, \mathrm{t})=-\frac{1}{2} \nabla^{2}+\mathrm{v}^{\mathrm{KS}}(\mathrm{r}, \mathrm{t}) \quad \Phi_{\mathrm{i}}(\mathrm{r}, \mathrm{t})
$$

where $\mathrm{v}^{\mathrm{KS}}$ is given by

$$
v^{K S}(r, t)=v_{\text {ext }}(r, t)+j(r, t)+\frac{\delta A_{x c}[\rho]}{\delta \rho(r, t)}
$$

where $v_{\text {ext }}$ contains $v_{n e}$ and the time-dependent perturbation. 
In TDDFT the action functional $A[\rho]$ (also called $x c$ kernel) has to be approximated because its form is not known. This is done through the Adiabatic Local Density Approximation (ALDA) where instead of the exact xc kernel the time-independent local xc potentials are utilized, which allow to work with ground-state xc potentials in a time-dependent manner. Another approximation so-called Casida equations is introduced as a convenient way of applying TD KS approach to calculate the transition energies and momenta of a given system by using time-independent singly excited KS configurations. ${ }^{60}$ In order to introduce Casida's approximation, it is convenient to represent TD KS equations in matrix form, where the density is expanded into the set of one-electron time-independent orbitals $\left\{X_{i}(r)\right\}$ :

$$
\rho(r, t)=\sum_{p, q} c_{p}(t) c_{q}^{*} X_{p}(r) X_{p}^{*}(r)=\sum_{p, q} P_{q p} X_{p}(r) X_{p}^{*}(r)
$$

In Eq. (2.43) $\mathrm{P}_{\mathrm{qp}}$ is time-dependent density matrix for which the matrix element of the KS operator will be denoted as $\mathrm{F}_{\mathrm{qp}}$. Using such representation the $\mathrm{KS}$ equations become

$$
\sum_{q} F_{q p} P_{q r}-P_{q r} F_{q r}=i \frac{\partial}{\partial t} P_{p r}
$$

Introduction of the linear-response theory requires oscillatory time-dependent perturbation with frequency $\omega$. From Perturbation Theory the expansions of the $P$ and $F$ matrices can be obtained.

$$
P_{p q}=P_{p q}^{(0)}+P_{p q}^{(1)}
$$

and

$$
F_{p q}=F_{p q}^{(0)}+F_{p q}^{(1)}
$$

where the idempotency condition applies

$$
\sum_{q} P_{q p}^{(0)} P_{q r}^{(0)}=P_{p r}^{(0)}
$$

The $\mathrm{P}^{(0)}$ and $\mathrm{F}^{(0)}$ terms in equations (2.45) and (2.46) represent the time independent unperturbed the KS Hamiltonian and density matrix of the ground sate, respectively. Substitution of Eq. (2.45) and Eq. (2.46) into Eq. (2.44), and considering only elements no higher 
than first order, leads to the coupled matrix equations (a.k.a., Casi da equations) ${ }^{60}$

$$
\begin{array}{ccc}
A & B & X_{I} \\
B^{*} & A^{*} & Y_{I}
\end{array} \quad \omega \begin{array}{ccc}
1 & 0 & X_{I} \\
0 & -1 & Y_{I}
\end{array}
$$

where $\omega$ denotes the vertical excitation energies and the matrices $A, B$ can be written in terms of the KS orbital energy differences and two-electron integrals (using Mulliken notation):

$$
\begin{gathered}
A_{i a, j b}=\delta_{i j} \delta_{a b}\left(\varepsilon_{a}-\varepsilon_{i}\right)+(i a \mid j b)+\left(i a\left|f_{x c}\right| j b\right) \\
B_{i a, j b}=(i a \mid b j)+\left(i a\left|f_{x c}\right| b j\right)
\end{gathered}
$$

with the usual notation for $\mathrm{i}, \mathrm{j}$, and a, b orbital s, i.e., occupied and virtual, respectively. $\varepsilon_{\mathrm{i}}$ and $\varepsilon_{a}$ are their respective orbital energies. $X$, and $Y$ are vector arrays whose $I$-th elements represents the excitation and deexcitation amplitudes, respectively.

The non-Hermitian eigenval ues of the Casida equations (Eq. (2.48)) remain the same, but their elements can be transformed into a Hermitian eigenval ue problem

$$
\Omega F_{I}=\omega_{l}^{2} F_{I}
$$

where $\Omega$ and $F_{I}$ are subjects of the following transformations

$$
\Omega=(A-B)^{1 / 2}(A+B)(A-B)^{1 / 2}
$$

and

$$
F_{I}=(A-B)^{1 / 2}\left(X_{1}+Y_{1}\right)
$$

The fact that the matrix difference is positive definite allows TDDFT to eval uate the transition energies and transition moments using time-independent quantities, i.e., the singly excited KS configurations. 


\subsection{Modern Coupled Cluster Methods and Density Func- tionals}

The goal of modern CC methods is to provide a good description of bond breaking, biradicals, and other systems involving large contribution of the non-dynamical correlation energy, with the simplicity of classical Single-Reference CC (SRCC) methods. This has resulted in the development of many CC methods based on non-iterative approaches; a few examples are provided by Gwal tney et. al., ${ }^{61}$ and further modified by Hirata and coworkers. $^{62}$ These approaches are called $\operatorname{CCSD}(2)$ and $\operatorname{CCSD}(2)_{T}$, where the last is particularly important for further discussion in this text (section 2.2.1 and Chapter3). Another encouraging idea is based on Methods of Moments of Coupled Cluster equations (MMCC), introduced and developed first by Kowal ski and Piecuch. ${ }^{63-66}$ This new methodology gives" the explicit formula for the noniterative correction that needs to be added to energy obtained in the standard approximated SRCC cal culations, such as CCSD or CCSDT, to recover the FCl result." 63,64

The conventional truncated $\mathrm{CC}$ approximates operator $\mathrm{T}$ by substituting it with $\mathrm{T}^{(\mathrm{A})}$, which includes chosen excitation level $\left(\mathrm{m}_{\mathrm{A}}\right)$, and it is given as

$$
\mathrm{T} \approx \mathrm{T}^{(\mathrm{A})}=\sum_{\mathrm{i}=1}^{\mathrm{m}_{\mathrm{A}}} \mathrm{T}_{\mathrm{i}}
$$

where $m_{A}<n, n$ is the number of correlated electrons in a given system, $T_{n}$ obeys to the formalism of second quantization, and has form of

$$
T_{n}=\sum_{\substack{i_{1}<\ldots<i_{n} \\ a_{1}<\ldots<a_{n}}} t_{a_{1} \ldots a_{n}}^{i_{1} \ldots i_{n}} a^{a_{1}} \ldots a^{a_{n}} a_{i_{n}} \ldots a_{i_{1}}
$$

In Eq. (2.55), $\mathrm{t}_{\mathrm{a}_{1} \ldots \mathrm{a}_{n}}^{\mathrm{i}_{1} \ldots \mathrm{i}_{\mathrm{n}}}$ are the relevant cluster amplitudes, $\mathrm{a}^{\mathrm{p}}$ with $\mathrm{p}=\mathrm{a}_{1}, \mathrm{a}_{2}, \ldots$ is the creation operator acting on unoccupied p spin-orbitals, $a_{r}$ with $r=i_{1}, i_{2}, \ldots$ is the annihilation operator related to occupied r spin-orbitals. 
The classical CC equations (independent from the energy) for this $\mathrm{T}^{(\mathrm{A})}$ operator can be written as

$$
\left\langle\Phi_{i_{1} \ldots i_{n}}^{a_{1} \ldots a_{n}}\left|\bar{H}^{(A)}\right| \Phi\right\rangle=0
$$

The $\bar{H}^{(A)}$ is the similarity-transformed Hamiltonian defined as

$$
\bar{H}^{(A)} \equiv e^{-T^{(A)}} H e^{T^{(A)}}=\left(H e^{T^{(A)}}\right) C
$$

where the superscript A indicates the level of CC theory ( 2 for CCSD, 3 CCSDT, etc.) , the subscript $C$ designates the connected part of the corresponding operator.

The energy correction of method A is

$$
\Delta E^{(A)} \equiv E^{(A)}-E^{H F}
$$

The construction of the correction $\delta^{(A)}$ in MMCC is designed to recover $\mathrm{FCl}$ results from the energy obtained with SRCC methods, and it is defined as

$$
\delta^{(A)} \equiv E-E^{(A)}
$$

where $E$ is the exact energy of the system $\left(\mathrm{FCl}\right.$ energy), and $\mathrm{E}^{(\mathrm{A})}$ is energy of the chosen SRCC method.

Eq. (2.59) is the fundamental equation of MMCC, and it can be expressed in terms of generalized moments $M{ }_{a_{1} \ldots a_{n}}^{i_{1} \ldots i_{n}}\left(m_{A}\right)$. One way to guide $\delta_{0}^{(A)}$ to reach the ground state $\left(E_{0}\right)$ is to utilize the asymmetric energy expression ${ }^{67}$ given below

$$
\mathrm{E}_{0}=\frac{\left\langle\Psi_{0}\left|\mathrm{H} \mathrm{e}^{T^{(A)}}\right| \Phi\right\rangle}{\left\langle\Psi_{0}\left|\mathrm{e}^{T(\mathrm{~A})}\right| \Phi\right\rangle}
$$

By applying $\mathrm{e}^{T^{(A)}} \mathrm{e}^{-T^{(A)}}=1$ to Eq. (2.60); Eq. (2.61) is obtained

$$
E_{0}=\frac{\left\langle\Psi_{0}\left|\mathrm{e}^{T^{(A)}} e^{-T^{(A)}} H^{T^{(A)}}\right| \Phi\right\rangle}{\left\langle\Psi_{0}\left|\mathrm{e}^{T^{(A)}}\right| \Phi\right\rangle}
$$


This gives the asymmetric energy expression in terms of the similarity-transformed Hamiltonian

$$
E_{0}=\frac{\left\langle\Psi_{0}\left|e^{T^{(A)}} \overline{H^{(A)}}\right| \Phi\right\rangle}{\left\langle\Psi_{0}\left|\mathrm{e}^{T^{(A)}}\right| \Phi\right\rangle}
$$

By using appropriately the resolution of identity in the $\mathrm{N}$-electron Hilbert space

$$
E_{0}=\frac{\left\langle\Psi_{0}\left|e^{T^{(A)}}\right| \Phi\right\rangle\left\langle\Phi\left|+\sum_{n=1}^{N} \sum_{\substack{i_{1}<\ldots<i_{n} \\ a_{1}<\ldots<a_{n}}}\right| \Phi_{i_{1} \ldots i_{n}}^{a_{1} \ldots a_{n}}\right\rangle\left\langle\Phi_{i_{1} \ldots i_{n}}^{a_{1} \ldots \ldots a_{n}}\left|\bar{H}^{(A)}\right| \Phi\right\rangle}{\left\langle\Psi_{0}\left|e^{T(A)}\right| \Phi\right\rangle}
$$

which can be rearranged as

$$
E_{0}=\frac{\left\langle\Psi_{0}\left|\mathrm{e}^{T^{(A)}}\right| \Phi\right\rangle\left\langle\Phi\left|\bar{H}^{(A)}\right| \Phi\right\rangle+\sum_{n=1}^{N} \sum_{\substack{i_{1}<\ldots<i_{n} \\ a_{1}<\ldots<}}\left\langle\Psi_{0}\left|e^{T(A)}\right| \Phi_{i_{1} \ldots i_{n}}^{a_{1} \ldots a_{n}}\right\rangle\left\langle\Phi_{i_{1} \ldots i_{n}}^{a_{1} \ldots a_{n}}\left|\bar{H}^{(A)}\right| \Phi\right\rangle}{\left\langle\Psi_{0}\left|e^{T(A)}\right| \Phi\right\rangle}
$$

Thenormal ization condition (Eq. (2.65)), must be postul ated in order to ensurethe recovery of the exact wave function $\left(\Psi_{0}\right)$ on the left hand side of the expression for the asymmetric energy. ${ }^{67}$

$$
\left\langle\Psi_{0} \mid \Psi_{0}^{(\mathrm{A})}\right\rangle \equiv\left\langle\Psi_{0}\left|\mathrm{e}^{T^{(\mathrm{A})}}\right| \Phi\right\rangle=1
$$

Due to the normal ization condition the denominator and the first term in the numerator of Eq. (2.64) vanish, giving the expression

$$
E_{0}=\left\langle\Phi\left|\bar{H}^{(A)}\right| \Phi\right\rangle+\sum_{n=1}^{N} \sum_{\substack{i_{1}<<<i_{n} \\ a_{1}<\ldots<a_{n}}}\left\langle\Psi_{0}\left|e^{T(A)}\right| \Phi_{i_{1} \ldots i_{n}}^{a_{1} \ldots a_{n}}\right\rangle\left\langle\Phi_{i_{1} \ldots i_{n}}^{a_{1} \ldots . a_{n}}\left|\bar{H}^{(A)}\right| \Phi\right\rangle
$$

where, the first term is the ground state energy of the "classical" $\operatorname{SRCC}\left(E_{0}^{(A)}\right)$ and the $\left\langle\Phi_{i_{1} \ldots i_{n}}^{a_{1} \ldots a_{n}}\left|H^{(A)}\right| \Phi\right\rangle$ are the generalized moments $\left(M_{a_{1} \ldots a_{n}}^{i_{1} \ldots i_{n}}\left(m_{A}\right)\right)$, which are projections of the $C C$ equations on the excited determinants. Keeping in mind that for $n \leq m_{A}, M_{a_{1} \ldots}^{i_{1} \ldots a_{n}}\left(m_{A}\right)=$ 0 the expression

$$
E_{0}=E_{0}^{(A)}+\sum_{n=m_{A}+1}^{N} \sum_{\substack{i_{1} \\ a_{1}<\ldots<i_{n}<\ldots}}\left\langle\Psi_{0}\left|e^{T(A)}\right| \Phi_{i_{1} \ldots i_{n}}^{a_{1} \ldots a_{n}}\right\rangle \times M_{a_{1} \ldots a_{n}}^{i_{1} \ldots i_{n}}\left(m_{A}\right)
$$

can be written. This is very similar to the original expression introduced first by Kowal ski and Piecuch ${ }^{63}$ via the energy functional. Furthermore, since the exact MMCC correction 
$\delta(A)$ is defined as the difference between the exact energy and the energy obtai ned from the SRCC approximated method (Eq. (2.59)), now it can be written in terms of the generalized moments. For the ground state case the exact MMCC correction

$$
\delta_{0}^{(A)}=\sum_{n=m_{A}+1}^{N} \sum_{\substack{i_{1}<\ldots<i_{n} \\ a_{1}<\ldots<a_{n}}}\left\langle\Psi_{0}\left|e^{T(A)}\right| \Phi_{i_{1} \ldots i_{n}}^{a_{1} \ldots a_{n}}\right\rangle \times M_{a_{1} \ldots a_{n}}^{i_{1} \ldots i_{n}}\left(m_{A}\right)
$$

Although the exact MMCC correction given in Eq. (2.68) is still very similar to the original formulation, ${ }^{63}$ here the denominator vanished due to the normalization condition introduced first in work by Piecuch and Wloch. ${ }^{67}$ The two-body interactions contained into the Hamiltonian condition strip the upper summation limit of Eq. (2.68) to $\mathrm{N}_{\mathrm{A}}$. At the same time the $n$-body moments $M_{a_{1} \ldots a_{n}}^{i_{1} \ldots i_{n}}$ with $n>N_{A}$ will vanish. Therefore, $\delta_{0}^{(A)}$ is in fact, independent from the number of electrons, and can be written as

$$
\delta_{0}^{(A)}=\sum_{n=m_{A}+1}^{N_{A}} \sum_{\substack{i_{1}<\ldots<i_{n} \\ a_{1}<\ldots<a_{n}}}\left\langle\Psi\left|e^{T(A)}\right| \Phi_{i_{1} \ldots i_{n}}^{a_{1} \ldots a_{n}}\right\rangle \times M_{\substack{a_{1} \ldots a_{n} \\ i_{1} \ldots i_{n}}}^{a_{1}}\left(m_{A}\right)
$$

In other words, the "classical" SRCC methods are obtained when the moments for $\mathrm{n}=$ $1, \ldots, m_{A}$ are imposed to vanish, and $N_{A} \geq n>m_{A}$ are not taken into account. For MMCC equations that is not the case. After determining $T^{(A)}$, by zeroing moments for $n=1, \ldots, m_{A}$, the remaining moments (for $n>m_{A}$ ) can be cal culated for determining $\delta^{(A)}$, which when added to the ground state energy of the "classical" SRCC will retrieve the exact ground state energy. The upper summation limit in Eq. (2.69) gives the maximum val ue of nonzero $n$-body moments. For example, for $m_{A}=2$, the maximum value of non-zero moments $N_{A}$ is six, and only $n=3,4,5,6$ moments are left.

In order to develop computationally efficient MMCC methods the n-body expansions for $\delta^{(A)}$ needs to be truncated at some low excitation level, labelled as $m_{B}$ (where the condition $m_{A}<m_{B} \leq N_{A}$ has to be satisfied); this leads to approximated MMCC approaches called $\operatorname{MMCC}\left(m_{A}, m_{B}\right)$. The Renormalized $(R)$ and Completely-Renormalized (CR) CC methods are the most noteworthy members of the $\operatorname{MMCC}\left(m_{A}, m_{B}\right)$ family; specifically these are 
$R-\operatorname{CCSD}(T), \operatorname{CR}-\operatorname{CCSD}(T), R-\operatorname{CCSD}(T Q)$ and $C R-\operatorname{CCSD}(T Q)$. Details about these methods can be found in work by Piecuch et al. ${ }^{66}$ and references therein and are beyond the aim of this work. Therefore, only the newest and more reliable approximations of MMCC reformulated through the left eigenstates of similarity-transformed Hamiltonian will be described (see next section). As a conclusive note it is worthwhile to stress that R-CC is a simplified version of CR-CC methods where the projections of the SRCC equations onto the excited configurations (i.e., moments) contain only the lowest-order terms of the twobody part of the Hamiltonian. ${ }^{63}$

\subsubsection{Essentials of the Bior thogonal M MCC Theory}

The reason behind developing another way to correct $\mathrm{CC}$ methods based on single reference is that despite the efforts in developing the truncated CC approaches discussed before, even sophisticated methods as CR-CCSD $(T)$ based on MMCC do not satisfy some of the desirable features for methods in quantum chemistry, i.e., to provide high accuracy for ground and excited states (including nearly-degenerated states and biradicals), to have manageable computational cost, and size-extensivity. CR-CCSD(T) and other similar methods are very good for describing single-bond breaking and biradical's reaction pathways ${ }^{68,69}$ but they are less accurate than "classical" $\operatorname{ccs}(T)$ in minimizing the energy. Besides they also minimally ( $\sim 5 \%$ of the total correlation energy ${ }^{70}$ ) break the size-extensivity of CC theory. To address these issues a new class of MMCC approaches involving single-reference, characterized by size-extensive $\mathrm{CC}$ with higher-than doubly ex-

cited noniterative corrections, has been proposed by Piecuch's group. ${ }^{67,71}$ This new class of $\mathrm{MMCC}$ approaches $\left(\mathrm{MMCC}_{\mathrm{L}}\right.$ ) arises from the biorthogonal $\mathrm{MMCC}$ formalism, which allows to treat the left (bra) and the right (ket) side of CC equations (or in case of excited states, EOMCC) separately. The MMCC $L$ approach with non-iterative triples, called CR$\mathrm{CC}(2,3)$ is so far the most significant method in this approach. CR-CC $(2,3)$ is at least as 
accurate as $\operatorname{CCSD}(T)$ for non-degenerated ground-state molecules, it is more accurate than $\operatorname{CCSD}(2)_{T}, \operatorname{CR}-\operatorname{CCSD}(T)$, and other similar methods in the biradical/bond stretching regions. Furthermore, it does not violate the size-extensivity, and its the cost is similar to $\operatorname{CcsD}(\mathrm{T}), \operatorname{CCSD}(2)_{\mathrm{T}}$ or $\mathrm{CR}-\operatorname{CCSD}(\mathrm{T}){ }^{67}$

The derivation of $C R-C C_{L}$ can be started from the asymmetric energy functional $E[\Psi]$ defined first by Kowal ski and Piecuch in 2000 (with the symbol $\wedge$ there). ${ }^{63}$

$$
E[\Psi]=\frac{\langle\Psi|H| \Psi C C\rangle}{\langle\Psi \mid \Psi C C\rangle}, \quad E\left[\Psi_{0}\right]=E_{0}
$$

where $\psi C C=\mathrm{e}^{\top(A)}|\Phi\rangle$ gives the exact ground state energy by solving the equation

$$
E_{0}=\frac{\left\langle\Psi_{0}\left|\mathrm{He}^{T^{(A)}}\right| \Phi\right\rangle}{\left\langle\Psi_{0}\left|\mathrm{e}^{T^{(A)}}\right| \Phi\right\rangle}
$$

As done before for MMCC, equation (2.71) can be modified into

$$
E_{0}=\frac{\left\langle\Psi_{0}\left|e^{T^{(A)}} \bar{H}^{(A)}\right| \Phi\right\rangle}{\left\langle\Psi_{0}\left|\mathrm{e}^{T^{(A)}}\right| \Phi\right\rangle}
$$

Substituting the "bra" (left) side with $\langle\Phi| \mathrm{L}_{0} \mathrm{e}^{-T^{(\mathrm{A})}}$

$$
E_{0}=\frac{\left\langle\Phi\left|L_{0} e^{-T^{(A)}} e^{T^{(A)}} \bar{H}^{(A)}\right| \Phi\right\rangle}{\left\langle\Phi\left|L_{0} e^{-T^{(A)}} e^{T^{(A)}}\right| \Phi\right\rangle}
$$

and since $\mathrm{e}^{T^{(A)}} e^{-T^{(A)}}=1$ the equation

$$
E_{0}=\frac{\left\langle\Phi\left|L_{0} \bar{H}^{(A)}\right| \Phi\right\rangle}{\left\langle\Phi\left|L_{0}\right| \Phi\right\rangle}
$$

is obtained.

Note that once again the normal ization condition (Eq. 2.65) is imposed to assure the $M M C C_{L}$ correction to properly restore the difference between $\mathrm{FCl}$ and $\mathrm{CC}^{(\mathrm{A})}$ energies.

Applying the resolution of identity in the $\mathrm{N}$-electron Hilbert space $\left(|\Phi\rangle\left\langle\Phi\left|+\sum_{n=1}^{N} \sum_{\substack{i_{1}<\ldots<i i_{n}<\ldots<a_{n} \\ a_{1}<}}\right| \Phi_{i_{1} \ldots i_{n}}^{a_{1} \ldots a_{n}}\right\rangle\left\langle\Phi_{i_{1} \ldots i_{n}}^{a_{1} \ldots a_{n}}\right|=1\right)$ to $\bar{H}^{(A)}$ in Eq. (2.74) and sorting it out 
gives

$$
E_{0}=\left\langle\Phi\left|L_{0}\right| \Phi\right\rangle\left\langle\Phi\left|\bar{H}^{(A)}\right| \Phi\right\rangle+\sum_{n=1}^{N} \sum_{\substack{i_{1}<\ldots<i_{n} \\ a_{1}<\ldots<a_{n}}}\left\langle\Phi\left|L_{0}\right| \Phi_{i_{1} \ldots i_{n}}^{a_{1} \ldots a_{n}}\right\rangle\left\langle\Phi_{i_{1} \ldots i_{n}}^{a_{1} \ldots a_{n}}\left|\bar{H}^{(A)}\right| \Phi\right\rangle
$$

where the first term equal s one (from the normal ization condition), the second is the ground state energy of method $A$, and the last is the non-iterative correction $\delta_{0}^{(A)}$ given by

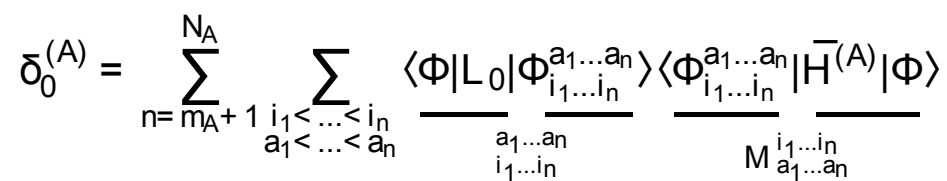

In Eq. (2.76) the upper and lower summation limits have been stripped. The upper and lower limits both vanish; the former due to the truncation level $\left(m_{A}<N\right)$, and the latter because of those elements with $n \geq m_{A}$. Therefore the final mathematical expression for the exact ground-state energy for the $\mathrm{CR}-\mathrm{CC}_{\mathrm{L}}$ approach is

$$
E_{0}^{C R-C C_{L}}=E_{0}^{C C}+\sum_{n=m_{A}+1}^{N_{A}} \sum_{\substack{i_{1}<\ldots<i_{n} \\ a_{1}<\ldots<a_{n}}} \underset{\substack{i_{1} \ldots i_{n} \\ a_{1} \ldots a_{n}}}{a_{1}^{i_{1} \ldots i_{n}}} M_{a_{1} \ldots a_{n}}\left(m_{A}\right)
$$

As an example let us consider the CR-CC $(2,3)$ method (known also as CR-CCSD $(T)_{L}$ ), for which the explicit equation for the ground-state energy is

$$
E_{0}^{[C R-C C(2,3)]}=E_{0}^{(C C S D)}+\sum_{\substack{i<j<k \\ a<b<c}} i_{i j k}^{a b c} M_{a b c}^{i j k}(2)
$$

where $\mathrm{M}_{\mathrm{abc}}^{\mathrm{ijk}}(2)$ corresponds to the projections of the $\mathrm{CC}$ equations on the excited determinants not included in the CCSD method, and it is given below as

$$
\begin{aligned}
M_{a_{1} \ldots a_{n}}^{i_{1} \ldots i_{n}}(2) & =\left\langle\Phi_{i_{1} \ldots i_{n}}^{a_{1} \ldots a_{n}}\left|\bar{H}^{C C S D}\right| \Phi\right\rangle \\
& =\left\langle\Phi_{i j k}^{a b c}\left|\left(H e^{T_{1}+T_{2}}\right) C\right| \Phi\right\rangle, \quad n=3
\end{aligned}
$$

Its general form is

$$
\begin{aligned}
M_{a_{1} \ldots a_{n}}^{i_{1} \ldots i_{n}}\left(m_{A}\right) & =\left\langle\Phi_{i_{1} \ldots i_{n}}^{a_{1} \ldots a_{n}}\left|\bar{H}^{(A)}\right| \Phi\right\rangle \\
& =\left\langle\Phi_{i_{1} \ldots i_{n}}^{a_{1} \ldots a_{n}}\left|\left(H e^{T^{(A)}}\right) C\right| \Phi\right\rangle
\end{aligned}
$$


The amplitudes $\underset{\mathrm{i}_{1} \ldots . . \mathrm{i}_{n}}{\mathrm{a}_{1} \ldots \mathrm{a}_{n}}$ defining the exact deexcitation operator $\mathrm{L}$, and the FCl "bra" satisfies the equation

$$
\langle\Phi| L \bar{H}^{(A)}=E_{0}\langle\Phi| L
$$

where

$L=L^{(A)}+\delta L^{(A)}=\sum_{n=0}^{m_{A}} L_{n}+\sum_{n=m_{A}+1}^{N} L_{n}$,

and

$$
L_{n}=\sum_{\substack{i_{1}<\ldots<i_{n} \\ a_{1}<\ldots<a_{n}}} i_{1} \ldots i_{1} \ldots a_{n} a^{i_{1} \ldots i_{n}} a_{a_{1} \ldots a_{n}}
$$

By decomposing the exact deexcitation operator $L$ into $L^{(A)}$ and $\delta L^{(A)}$ the first part can be solved quite easily for method $A$, by employing the deexcitation operator $L^{(A)}$

$$
L^{(A)} \quad L^{(A)}=1+\Lambda_{1}+\Lambda_{2}+\ldots+\Lambda_{m A}
$$

where, the operator $\Lambda^{(A)}$ is so-called "lambda" operator, ${ }^{72}$ and $L^{(A)}=L^{(A)}$ when $m_{A}=N$. $\delta L^{(A)}$ may al so be truncated at some low level, noted $m_{B}$, as shown below

$$
\delta L^{(A)} \approx \sum_{n=m_{A}+1}^{m_{B}} L_{n}
$$

This leads to the approximated form of $L$

$$
L \approx 1+\Lambda_{1}+\ldots+\Lambda_{m_{A}}+\sum_{n=m_{A}+1}^{m_{B}} L_{n}
$$

where

$$
L_{n}=\sum_{\substack{i_{1}<\ldots<i_{n} \\ a_{1}<\ldots . a_{n}}} i_{1} i_{1} \ldots a_{n} a_{n} a^{i_{1} \ldots i_{n}} a_{a_{1} \ldots a_{n}}, \quad n=m_{A}+1, \ldots, m_{B}
$$

Thereforethe desi red correction $\delta_{0}\left(m_{A}, m_{B}\right)_{L}$ can be obtained via the general ized moments $M{ }_{a_{1} \ldots a_{n}}^{i_{1} \ldots i_{n}}\left(m_{A}\right)$ defined as follows

$$
\delta_{0}\left(m_{A}, m_{B}\right)_{L} \equiv \sum_{n=m_{A}+1}^{m_{B}} \sum_{\substack{i_{1}<\ldots<i_{n} \\ a_{1}<\ldots<a_{n}}} \underset{\substack{i_{1} \ldots i_{n} \\ i_{1} \ldots a_{n}}}{a_{1}} M_{a_{1} \ldots a_{n}}^{i_{1} \ldots i_{n}}\left(m_{A}\right)
$$


For the CR-CC $(2,3)$ ground state

$$
\left\langle\Phi\left|L_{0} \bar{H}^{C C S D}\right| \Phi_{i j k}^{a b c}\right\rangle=E_{0}^{C C S D ~} \underset{i j k}{a b c}
$$

where $L_{0} \approx L^{(C C S D)}+L_{3}$, which leads to the expression

$$
\left\langle\Phi\left|L^{(C C S D)} \bar{H}^{(C C S D)}\right| \Phi_{i j k}^{a b c}\right\rangle+\sum_{\substack{l<m<n \\ d<e<f}}\left\langle\Phi_{I m n}^{\text {def }}\left|\bar{H}^{C C S D}\right| \Phi_{a b c}^{i j k}\right\rangle \underset{I m n}{\operatorname{def}}=E_{0}^{C C S D} \underset{i j k}{a b c}
$$

solving Eq. (2.89) for the amplitudes $\underset{\mathrm{abc}}{\mathrm{ijk}}$ gives

$$
\begin{aligned}
i_{a b c}^{i j k} & =\frac{\langle\Phi|\left(1+\Lambda^{C C S D} \bar{H}^{C C S D}\left|\Phi_{i j k}^{a b c}\right\rangle\right.}{D_{a b c}^{i j k}} \\
D_{a b c}^{i j k} & =E_{0}^{C C S D}-\left\langle\Phi_{i j k}^{a b c}\left|\bar{H}^{C C S D}\right| \Phi_{i j k}^{a b c}\right\rangle
\end{aligned}
$$

The CR-CC $(2,3)$ method can al so be applied to open-shell systems by employing the ROHF reference wave function. A benchmark study showing the applicability of this method to such systems can be found, for example, in the paper by Wloch.et al. ${ }^{73}$ While the discussion above has focused on the electronic ground-state, in some cases the excited states may be of interest in a given chemical problem. Excited states of different symmetry than the ground state can be handled as easily as the ground state by using standard post-HF methods. However, if these systems are open-shell a multireference wave function for the correct zeroth-order description might be necessary. ${ }^{6}$ Another problem can occur: using augmented basis sets may be unnecessary to describe the ground state. On the contrary electronically excited states are much more diffused than the ground state, and as such they require bigger and therefore more expensive basis set (one which include $d$ - and other diffuse functions). Additionally, for excited states with low energy and the same symmetry of the ground-state the situation is harder because HF and other variational methods will simply find the lowest possible solution of the Shrödinger equation, i.e., the ground state instead of desired excited state.

The computational method which is based on single-reference wave functions and presents 
very convenient approach to excited states is the Equation-of-Motion Coupled Cluster method (EOMCC) ${ }^{74-78}$ The main idea behind EOMCC is to calculate the vertical excitation energy $\left(\omega_{\mu}\right)$ that al lows to determine the excited-state wave function $\Psi_{\mu} . \omega_{\mu}$ is the difference between excited and ground state energies, denoted in the equation below as $E_{\mu}$ and $\mathrm{E}_{0}$, respectively

$$
\omega_{\mu} \equiv E_{\mu}-E_{0}
$$

A vertical excitation energy for a given system is typically found by solving the truncated EOMCC equations at some lower level (A)

$$
\left\langle\Phi_{i_{1} \ldots i_{n}}^{a_{1} \ldots a_{n}}\left|\left(H_{o p e n}^{(A)} R_{\mu, o p e n}^{(A)}\right) C\right| \Phi\right\rangle=\omega_{\mu}^{(A)} r_{\mu, i_{1} \ldots i_{n}}^{a_{1} \ldots a_{n}}
$$

where $r_{\mu, i_{1} \ldots i_{n}}^{a_{1} \ldots a_{n}}$ are excitation amplitudes, and $R_{\mu, o p e n}^{(A)}$ and $\bar{H}^{(A)}$ correspond to the "open" diagrams of $\bar{H}$ and $R_{\mu}$, i.e. diagrams with the external Fermion lines ${ }^{70}$

The $R_{\mu}^{(A)}$ term is the linear excitation operator that generates the excited state function

$$
\left|\Psi_{\mu}\right\rangle=R_{\mu} e^{\top}|\Phi\rangle
$$

where

$$
R_{\mu}=R_{\mu, 0}+\sum_{n=1}^{N} R_{\mu, n} \equiv r_{\mu, 0} 1+\sum_{n=1}^{N} \sum_{\substack{i_{1} 1<\ldots<i_{n} \\ a_{1}<\ldots<a_{n}}} r_{\mu, i_{1} \ldots \ldots}^{a_{1} \ldots a_{n}} a^{i_{1} \ldots . i_{n}} a_{a_{1} \ldots a_{n}}
$$

In Eq. (2.94) the operator 1 is the unit operator, and by defining $R_{\mu=0}=1$ the excited wave function (Eq. 2.93) can be applied the both ground state $\mu=0$ and excited states $\mu>0$. By solving the system of EOMCC equations (2.92) $R_{\mu, \text { open }}$ and $\omega_{\mu}$ are calculated, and $r_{\mu, 0}$ for the reference determinant $|\Phi\rangle$ is obtained by solving following equation

$$
r_{\mu, 0}=\frac{\left\langle\Phi\left|\left(\bar{H}_{\text {open }} R_{\mu, o p e n}\right) C\right| \Phi\right\rangle}{\omega_{\mu}}
$$

Final ly, the operator $R_{\mu}$ is known and $\left|\Psi_{\mu}\right\rangle$ can be determined.

Standard truncated EOMCC methods such as EOMCCSD or EOMCCSD(T) encounter problems with two-electron (or in general many-electron) transitions arising from the fact 
that their ground-states are equival ent. In case of EOMCCSD this is due to a lack of higher cluster operators (i.e., mainly $T_{3}$ and $T_{4}$ ), that are crucial to correctly describe excited states having a dominating doubly excited character and/or bond breaking coordinates al ong the PES. Although EOMCCSD $(T)$ approximates effects of $T_{3}$ cluster, this method also fails because the MBPT arguments are used to account for triples. ${ }^{66}$ The Methods of Moments theory significantly improves ground-state truncated SRCC methods such as $\operatorname{CCSD}(T)$, therefore it represents the choice to improve truncated EOMCC methods.

MMCC theory can be applied to excited-states ${ }^{66,79-81}$ by following the same procedure as for the ground-state $\mathrm{CC}$ methods, the only difference being that now the exact excited-state energy $\left(E_{\mu}\right)$ is recovered. This is done by adding the excited-state noniterative correction $\delta_{\mu}^{(A)}$ (containing the generalized moments of the EOMCC equations) to the excited-state energy obtained from some low level EOMCC method, denoted by $m_{A}$ ( $A$ is as usual the level of the truncation). The more general biorthogonal MMCC formalism ( $\left.M M C C_{L}\right)$ discussed before has an advantage over the original MMCC in the finite $\delta_{0}^{(A)}$ expansion (see Eq. (2.76)) and the same is true when $M M C C_{L}$ is applied to the formula defining $\delta_{\mu}^{(A)} 82$

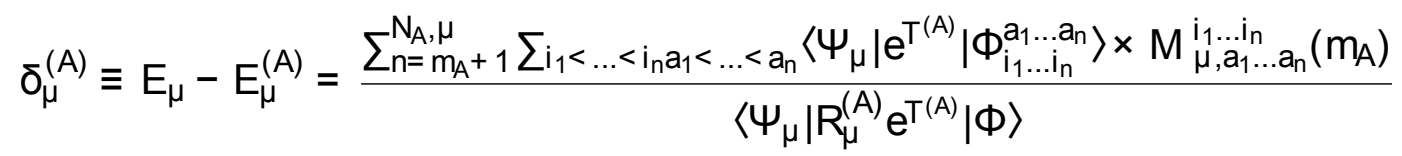

The number of generalized moments $\left(M_{\mu, a_{1} \ldots a_{n}}^{i_{1} \ldots i_{n}}\left(m_{A}\right)\right)$ in Eq. (2.96) is independent from the number of electrons in the system (which is emphasized by the upper summation limit $\mathrm{N}_{\mathrm{A}}$ ), but it has to be lowered in order to reduce the cost of calculations. This reduction is determined by the choice of the $m_{B}$ level and it leads to approximated noniterative corrections $\delta_{\mu}\left(m_{A}, m_{B}\right)$, which are more competitive for practical applications of the excited-state MMCC $C_{L}$ methods. The CR-EOMCC $(2,3)$ approach $^{82,83}$ (also abbreviated as $\mathrm{CR}$-EOMCCSD $\left.(T)_{L}\right)$ is an excellent example where the number of generalized moments in $\delta(2,3)$ is reduced from 8 to 3 . This method can be seen as a reformulated version of CR-EOMCCSD $(T)$ first introduced by Piecuch et al. ${ }^{81}$ The CR-EOMCC $(T)$ eliminates 
failures of "classical" $\operatorname{EOMCCSD}(T)$, which (as mentioned before) ultimately results from the perturbative form of triple excitations, that are ineffective for describing excited states dominated by electron-pair excitations and larger area of excited-state potential energy surfaces. ${ }^{84}$ Despite this success, the CR-EOMCCSD(T) method suffers from small size extensivity errors, which affect the amount of recovered total correlation energy.

The explicit CR-EOMCCSD(T) energy formula (Eq. (2.97)) redefined utilizing MMCC theory leads to more accurate description of the excited states, especial ly for the open-shell systems dominated by double excitations:

$$
E_{\mu}(2,3)=E_{\mu}^{(\text {EOMCCSD })}+\sum_{\substack{i<j<k \\ a<b<c}} \frac{\left\langle\Psi_{\mu}\left|M_{\mu, a b c}^{i j k}(2)\right| \Phi_{i j k}^{\mathrm{abc}}\right\rangle}{\left\langle\Psi_{\mu}\left|R_{\mu}^{(A)} \mathrm{e}^{T_{1}+T_{2}}\right| \Phi\right\rangle}
$$

where the moments $M_{\mu, a b c}^{i j k}(2)$ can be written as

$$
M_{\mu, a b c}^{i j k}(2)=\left\langle\Phi_{i j k}^{a b c}\left|\left(\bar{H}^{(C C S D)} R_{\mu}^{(C C S D)}\right)\right| \Phi\right\rangle
$$

The reformulated expression for $\mathrm{E}_{\mu}(2,3)$, which is the key idea behind the CR-EOMCC $(2,3)$ scheme $e^{82,83}$ is shown in Eq. (2.99), as an example of $M M C C\left(m_{A}, m_{B}\right) L$ methodology for excited states:

$$
E_{\mu}(2,3)=E_{\mu}+\delta_{\mu}(2,3)
$$

where $\delta_{\mu}(2,3)=\sum_{\substack{i<j<k \\ a<b<c}}^{a b, i j k} M_{\mu, a b c}^{i j k}(2)$.

The triply excited moments $M_{\mu, a b c}^{i j k}(2)$ are determined using the expression

$$
M_{\mu, a b c}^{i j k}(2)=\left\langle\Phi_{i j k}^{a b c}\left|H^{(C C S D)}\left(R_{\mu, 0}+R_{\mu, 1}+R_{\mu, 2}\right)\right| \Phi\right\rangle
$$

Note that the $\delta_{\mu}^{(\mathrm{A})}$ given in Eq. (2.96) is more general for the noniterative corrections because it can be reduced to the ground state. Its formula in the $\mathrm{MMCC}\left(\mathrm{m}_{\mathrm{A}}, \mathrm{m}_{\mathrm{B}}\right)_{L}$ is

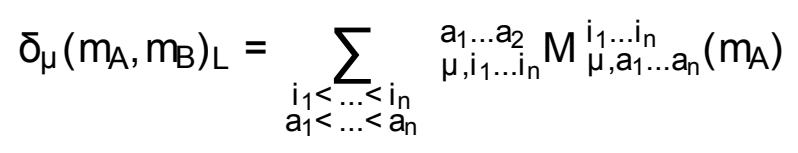


where the normalization condition (Eq. (2.102)) has to be maintained in order to recover the $\mathrm{FCl}$ energy $E_{\mu}$ :

$$
\left\langle\Phi\left|L_{\mu}^{(A)} R_{\mu}^{(A)}\right| \Phi\right\rangle=1
$$

Eq. (2.102) gives expression in Eq. (2.65) when $\mu=0$ and $R_{\mu}^{(A)}=1$.

Furthermore, Eq. (2.102) is similar to the original biorthogonal condition

$$
\left\langle\Phi\left|L_{\mu}^{(A)} R_{v}^{(A)}\right| \Phi\right\rangle=\delta_{\mu, v}
$$

which describes the excited left states, obtained utilizing the deexcitation operators $L_{\mu}^{(A)}$, defined by their amplitudes $\underset{\mu, \dot{i}_{1} \ldots i_{n}}{a_{1} \ldots a_{n}}$. More details about different CR-EOMCC $(2,3)$ variants can be found in recent work by Wloch et al. 83

The importance of EOMCC theory is not limited to its capability of cal culating the vertical excitation energies $\omega_{\mu}$, but it also can be applied to obtain Ionization Potentials (IP) and Electron Affinities (EA). Therefore, when one wishes to explore the EOMCC formalism, the following abbreviations are usual ly used: EE-EOMCC, IP-EOMCC, and EA-EOMCC. These reflect the ability of the method to obtain: excitation energy, ionization-potential, and electron affinities cal culations, respectively (numerical examples are reported by Piecuch et al. ${ }^{82}$ ). The EOMCC methodology can be seen as reformulation of the CC Linear Response Theory (CC-LRT), ${ }^{85-87}$ and both are theoretically identical to earlier developed Spin-Adapted Cluster Configuration Interaction (SAC-Cl). ${ }^{88}$ This last method has been extended to SAC-Cl general-R method ${ }^{89,90}$ for processes involving multi-electron excitations as often found in open-shell of excited state systems. ${ }^{88}$ Final ly, other promising CC methods for excited states worth mentioning have been developed by Krylov et al. These are the Spin-Flip Coupled Cluster, ${ }^{16,91,92}$ and the spin-conserving EOMCC, ${ }^{93}$ al though the latter reprises ideas contained in earlier developed CR-EOMCC $(2,3) .{ }^{82}$ 


\subsubsection{Beyond Standard Exchange-Cor relation Functionals}

Relevant recent development in DFT are based on a few key ideas which can be used to categorized functional s into: parameterized, adiabatic connection, local hybrid, double hybrid and long-range corrected. ${ }^{42}$ The first group is a continuation of a very well established idea of constructing the $x c$ functional using some number of empirical parameters that are fitted to match databases, for example, experimentally found heats of formation for organic compounds. The advance beyond popular fitting done in the past, such as for B3LYP 54,56 (three parameters adjusted to reproduce experimental data set $\mathrm{G} 1$ ), is the use of a much larger library containing chemical species of importance. This approach led to the development of some very successful functionals, as the Truhlar's group M06 functional family. ${ }^{94}$ Despite how well behaving parameterized functionals may be the outcome of the fitting procedure does not address an important issue, i.e., the number of parameters necessary to find the mathematical formula which gives that of the "exact" functional.

The so-cal led adiabatic connection ${ }^{95}$ is a method which allows to continuously transform a model system into a physical system. In other words a standard xc functional can be viewed as a very basic link or an adiabatic connection between the model system consisting of the noninteracting particles. Such model is enforced to produce properties of a real system such as ground-state energy and density. ${ }^{96}$ Hence, either the model system or a method of building a functional can be modified so that a better adiabatic connection can be ensured, and the description of the real system can improve. The second approach, i.e., constructing a functional by employing a model for the adiabatic connection integrant $\left(E_{x c}={ }_{0}^{1} W_{\lambda} d \lambda\right.$ where $\left.W_{\lambda}=\left\langle\Psi_{\lambda}\left|V_{e}\right| \Psi_{\lambda}\right\rangle-J[\rho]\right)$, blossomed over the last few years. ${ }^{42}$ Yet, a somewhat similar problem occurs as for the parameterized functionals the number of ways to exploit the link might be endless. Nevertheless, this development is very promising and in the future it may provide answers for some of the main chal lenges of DFT such as limitations in deal ing with strongly correlated systems (for more details see work by Cohen et al. ${ }^{42}$ and 
references therein).

Functionals involving unoccupied orbitals and eigenvalues are called double hybrids because they are based on the key idea of mixing the standard GGA xc functional with a fraction of HF exchange and a perturbative second-order correlation generated from these KS orbitals and eigenvalues. These orbitals and eigenvalues are different from those produced by MP2 (Chapter 2, Section 2.1.2), because they arise from a local potential instead from HF. Therefore, one-electron excitations introduce a small contribution. An example of double-hybrid functional is B2PLYP97

$$
E_{x C}^{B 2 P L Y P}=\left(1-a_{x} E_{x}^{G G A}+a_{x} E_{x}^{H F}+\left(1-a_{c}\right) E_{c}^{G A A}+a_{c} E_{c}^{M P 2}\right.
$$

with

$$
\mathrm{E}_{\mathrm{c}}^{\mathrm{PT} 2}=\frac{1}{4} \sum_{\mathrm{ia}} \sum_{\mathrm{jb}} \mathrm{t}_{\mathrm{ab}}^{\mathrm{ij}} \mathrm{K}_{\mathrm{ab}}^{\mathrm{ij}}
$$

where $K_{a b}^{i j}$ are the antisymmetrized two el ectron exchange integrals and $t_{a b}^{i j}$ are the doubles excitation amplitudes.

A new idea is based on using the fluctuation dissipation theorem ${ }^{98}$ where the correlation energy of the ground-stateis now expressed as an integral running over thefrequency which depends on many-body as well as single-particle excitations. This dependence is expressed by the random phase approximation (RPA $)^{42}$

$$
E_{c}^{R P A}=\sum_{i a} \omega_{i a}-A_{i a, i a}
$$

\subsubsection{L ong-Range Cor rected DFT}

The last recent development is given by the Long-Range Corrected (LRC) xc functionals. Since electron interactions in the long-range are not treated properly by standard xc functionals an improper treatment of Rydberg states as well as large errors for CT transitions can occur. ${ }^{60,99,100}$ In fact in the standard xc functionals the asymptotic decay of the xc 
potential $\left(v_{x c}\right)$ is too rapid, i.e., $\lim _{r \rightarrow \infty} v_{x c}(r)=-\frac{1}{r}$. One of the most promising remedies to such issue is to increasingly substitute the long-range part of the density exchange with HF exchange. This originates ${ }^{101,102}$ from the idea of separating the range of the electronelectron interactions into two parts: short- and long-range. The range separation depends on the error function given by the equation

$$
\frac{1}{r_{12}}=\frac{\operatorname{erfc}\left(\mathrm{rr}_{12}\right)}{\mathrm{r}_{12}}+\frac{\operatorname{erf}\left(\mathrm{\gamma r}_{12}\right)}{\mathrm{r}_{12}}
$$

which represents a partitioning of the total exchange energy into short- (first term) and long-range (second term) contributions where the er $f$ term is the error function, $y$ is the range separation parameter and $r_{i j}$ is the distance between electron $\mathrm{i}$ and electron $\mathrm{j}$.

The long-range $H F$ exchange $\left(E_{x}^{\mathrm{LR}-H F}\right)$ is defined $\mathrm{as}^{42}$

$$
E_{\mathrm{x}} \equiv-\frac{1}{2} \sum_{\mathrm{ij \sigma} \sigma} \frac{\Phi_{\mathrm{i \sigma} \sigma}^{*}\left(\mathrm{r}_{1}\right) \Phi_{\mathrm{j} \sigma}\left(\mathrm{r}_{1}\right) \operatorname{erf}\left(\mathrm{\gamma r}_{12}\right) \Phi_{\mathrm{j \sigma}}^{*}\left(\mathrm{r}_{2}\right) \Phi_{\mathrm{i \sigma}}\left(\mathrm{r}_{2}\right)}{\left|\mathrm{r}_{1}-\mathrm{r}_{2}\right|} \mathrm{dr}_{1} \mathrm{dr} \mathrm{r}_{2}
$$

and the long-range Density Functional Approximation (DFA) exchange $\left(E_{x}^{L R-D F A}\right)$ is calculated using its explicit formula.

Equation (2.107) was later generalized by Yanai et al. ${ }^{103}$ who introduced two extra parameters which allow to manipulate the amount of contribution from the HF exchange in the short- and long-range regions and to study the importance of $E_{x}^{\mathrm{HF}}$ over the whole range. This general ized form of the Coulomb potential $\left(1 / r_{12}\right)$ is given by

$$
\frac{1}{r_{12}}=\frac{1-\left[\alpha+\beta \cdot \operatorname{erf}\left(\gamma r_{12}\right)\right]}{r_{12}}+\frac{\alpha+\beta \cdot \operatorname{erf}\left(\gamma r_{12}\right)}{r_{12}}
$$

with $0 \leq \alpha+\beta \leq 1,0 \leq \alpha \leq 1$ and $0 \leq \beta \leq 1$ where $\alpha$ and $\alpha+\beta$ define the fraction of $\mathrm{HF}$ exchange at $r_{12}=0$ and $r_{12}=\infty$, respectively.

Hence the LRC-DFA can be described by the expression ${ }^{104}$

$$
E_{x C}^{L R C-D F A}=E_{c}^{D F A}+C_{H F} E_{x}^{S R-D F A}+\left(1-C_{H F}\right) E_{x}^{S R-D F A}(Y)+E_{x}^{L R-H F}(Y)
$$


where first term is the correlation (c), usual ly provided by GGA, the second is the exchange (x) functional with a fraction of HF exchange (defined by the $\mathrm{C}_{\mathrm{HF}}$ coefficient). The last two terms represent the short- (SR) and long-range (LR) components of the Coulomb potential given in Eq. (2.109).

While discussing LR corrected scheme it is important to al so mention the derivative discontinuity, ${ }^{105}$ which is related to another worth mentioning term, the so-cal led Self-Interaction Error (SIE). ${ }^{106}$ These will be briefly discussed next starting with the latter. Traditionally the "one-electron SIE" 107 is defined as the "inexactness of the one-electron systems" . ${ }^{106}$ In other words "one-electron SIE" is the spurious el ectron self-repul sion caused by the use of standard xc functional approximations. ${ }^{108}$ Apart from the "one-electron SIE", which can be eliminated, the so-called "many-electron SIE" is al so present (see for example work of Körzdörfer, et al. ${ }^{112}$ ). The consequences of the latter can be readily seen when one considers the trend representing the change of the total ground state energy $(\Delta \mathrm{E})$ versus the integer electron number $(\mathrm{N})$. Basically, in the exact DFT theory ${ }^{109}$ this trend for an open system (system that is allowed to exchange electrons with the environment) is a straight line. Furthermore, the linear dependence must be segmented by the derivative discontinuities at each integer. This is necessary because going from the neutral $(\Delta N=0)$ to the cation (the electron deficient portion of the plot with $\Delta N<0$ ) or from the neutral to the anion (the electron rich region with $\Delta \mathrm{N}>0$ ) the el ectron number varies by a small fraction $(\eta)$ and the energy jump occurs. In other words the derivative discontinuity defined for the $E_{x c}[\rho]$ as $^{110}$

$$
\Delta_{x C}=\left.\lim _{\eta \rightarrow 0^{+}} \frac{\delta E_{x} C[\rho]}{\delta \rho(r)}\right|_{N+\eta}-\left.\frac{\delta E_{x} C[\rho]}{\delta \rho(r)}\right|_{N+\eta}
$$

should be nonzero.

At an intuitive level this requirement is understandable since input of energy is necessary to remove or attach an electron. However, due to the "many-electron SIE" the trend $\Delta \mathrm{E}$ vs. $\mathrm{N}$ (or more specifically $\Delta \mathrm{N}$ ) does not represent a straight line, instead for standard 
xc functionals it curves either up or down depending on the delocalization error, i.e., the positive curvature occurs when too little delocalization is present and the negative in case of too much del ocal ization. ${ }^{111}$ The " many-electron SIE" can be minimized for example by utilizing "first-principles" tuning for the long-range corrected hybrid functional s. ${ }^{112}$ Note that this method will be shortly discussed later (Chapter 4, Sections 4.2.1.1 and 4.2.2). 


\section{Chapter 3}

\section{The Size Extensivity Problem in Excited-State EOMCC Methods ${ }^{1}$}

\subsection{Brief Introduction}

The size extensivity error (i.e., an error due to the energy not scaling linearly with increasing number of electrons) has been eliminated from the ground-state Methods of Moments of Coupled Cluster equations (MMCC) methods, such as CR-CCSD(T), ${ }^{67}$ by employing the biorthogonal MMCC (viz. MMCC $\mathrm{L}$ ) formalism. ${ }^{71,83}$ The success of the $\mathrm{CR}-\mathrm{CC}(2,3)$ scheme was one of the main reasons to extend the $\mathrm{MMCC}_{\mathrm{L}}$ method to electronic excitedstates, i.e., $C R-E O M C C\left(m_{A}, m_{B}\right)$ methods (for more details see section 2.2.1). Size extensivity is required for a proper description of some important processes. For instance, the size extensivity error exceeding the chemical accuracy $(1 \mathrm{kcal} / \mathrm{mol})$ can seriously affect accuracy of computed singlet-triplet energy gaps or dissociation energies. ${ }^{114}$

\footnotetext{
${ }^{1}$ The material contained in this Chapter was prepared in collaboration with N. Bauman. ${ }^{113}$
} 
The size extensivity problem of the CR-EOMCCSD $(T)$ method, which is an analog of $\mathrm{CR}-\operatorname{CCSD}(\mathrm{T})$ for the excited-states and the precursor of the $\operatorname{CR}-\operatorname{EOMCC}(2,3)$ approach, has been examined by Wloch et al., ${ }^{70}$ where it has been shown that cal cul ated excited-state energies obtained with the most accurate CR-EOMCCSD(T),ID approach introduce relatively small size extensivity errors (at least for systems up to about 50 correl ated electrons). Furthermore a significant improvement is seen compared to other tested methods involving approximated triples corrections, i.e., EOMCCSDT-1 and CC3, especially for states dominated by two electron excitations. Note that such states cannot be properly described by EOMCCSD, even though this method is size intensive. In case of vertical excitation energies the size-extensive quantity was al so numerical ly tested for the noninteracting $\mathrm{C}_{2}$ $+n N e(n=1,2,3)$ systems, which if correctly described should not depend on $n$. Vertical excitation energies of the lowest excited states cal culated for these systems with the CREOMCCSD(T),ID approach are superior in comparison to val ues obtai ned viaEOMCCSD, CC3 and EOMCCSDT-1afor the doubly exited states. Moreover, in spite of lack of the size intensivity of this approach, the accuracy of predicted vertical excitation energies for excited states dominated by double excitations is either exceeding or similar to the accuracy of computed vertical excitation energies of the singly excited states. ${ }^{70}$

\subsection{Motivation and Methodology}

Hirata and coworkers developed the EOM-CCSD $(2)_{\top}{ }^{115}$ method, which is capable of providing size-intensive excitation energies. Nevertheless the authors themselves admit that enforcing cancelation between the zero-body components of the second-order triples cor-

rection to the excited-state $\left(E_{\mu}^{(T 0)}\right)$ and the ground-state $\left(E_{0}^{(T 0)}\right)$, is an ad hoc solution as it is "neither rigorous nor accurate". ${ }^{115}$ An thorough analysis presented in a later study by Piecuch's group ${ }^{82}$ provides some insights into size extensivity problem of CR$\operatorname{EOMCC}(2,3)$, but dilemma on how to remove the error associated with this problem re- 
mains unsolved. Moreover, that work shows that Hirata's recipe to restore strict size intensivity in the approximated EOMCC approaches involving triples corrections to the total correlation energy is not what we could call a logical and analytical way to improve on this complex issue. It become clear that the reason for not retaining the size intensivity of excitation energies obtai ned with CR-EOMCC $(2,3)$ and other closely related approaches is due to " the presence of the size-extensive $\left(r_{\mu, 0}=0\right)$ or approximately size-extensive (non-zero $\left.r_{\mu, 0}\right)$ contribution" 82 indicated as $\beta_{\mu}(2,3)$ :

$$
\beta_{\mu}(2,3) \equiv \sum_{\substack{i<j<k \\ a<b<c}}\left(r_{\mu, 0} \underset{\mu, i j k}{a b c}-\underset{0, i j k}{a b c}\right) M_{0, a b c}^{i j k}(2)
$$

where $r_{\mu, 0}$ is the amplitude describing the zero-body component $\left(R_{\mu, 0}\right)$ of the operator $\mathrm{R}_{\mu}^{C C S D}$, whose general expression is given in Eq. (2.94). $\underset{\mu, \mathrm{ijk}}{\mathrm{abc}}$ and $\underset{0, \mathrm{ijk}}{\mathrm{abc}}$ arethede-excitation amplitudes that enter the triples corrections $\delta_{\mu}(2,3)$ of the excited-state and $\delta_{0}(2,3)$ of the ground-state, respectively. The $\mathrm{M}_{0, \mathrm{abc}}^{\mathrm{ijk}}(2)$ are triply excited moments of the ground-state CCSD equations.

The vertical excitation energy $\omega_{\mu}^{\mathrm{CR}-\operatorname{EOMCC}(2,3)}$ is the energy difference between the energy of a given excited state and the ground-state,

$$
\omega_{\mu}^{\mathrm{CR}-\operatorname{EOMCC}(2,3)} \equiv \mathrm{E}_{\mu}^{\mathrm{CR}-\operatorname{EOMCC}(2,3)}-\mathrm{E}_{0}^{\mathrm{CR}-\mathrm{CC}(2,3)}
$$

and it can be written as

$$
\omega_{\mu}^{\mathrm{CR}-\operatorname{EOMCC}(2,3)}=\omega_{\mu}^{\mathrm{CCSD}}+\alpha_{\mu}(2,3)+\beta_{\mu}(2,3)
$$

where $\omega_{\mu}^{\mathrm{CCSD}}$ is the vertical excitation energy of EOMCCSD, $\beta_{\mu}(2,3)$ in the equation (3.1), and $\alpha_{\mu}(2,3)$ term is given by

$$
\alpha_{\mu}(2,3)=\sum_{\substack{i<j<k \\ a<b<c}}^{a b c} \tilde{\mu}_{\mu, i j k}^{i j, a b c} \tilde{M}_{\mu, a b}^{i j k}(2)
$$

with $\tilde{M}_{\mu, \mathrm{abc}}^{\mathrm{ijk}}(2)=\left\langle\Phi_{\mathrm{ijk}}^{\mathrm{abc}}\left|\bar{H}^{\mathrm{CCSD}}\left(R_{\mu, 1}+R_{\mu, 2}\right)\right| \Phi\right\rangle$. 
Note that both $\omega_{\mu}^{\mathrm{CCSD}}$ and $\alpha_{\mu}(2,3)$ are size intensive, but $\beta_{\mu}(2,3)$ is not. Additionally, the later cannot be eliminated from $\omega_{\mu}^{\mathrm{CR}-\operatorname{EOMCC}(2,3)}$ unless its contribution to the $\delta_{\mu}(2,3)$ of $E_{\mu}^{C R-E O M C C}(2,3)$ is enforced to cancel out with the contribution to the $\delta_{0}(2,3)$ of $E_{0}^{C R-E O M C C}(2,3)$. In other words by eliminating the $\beta_{\mu}(2,3)$. In fact in doing so we can obtain a modified CR-EOMCC(2,3)A approach called here H-CR-EOMCC(2,3)A, which produces exactly the same results as the EOM-CCSD(2)T method. Hence, removing of the ground-state contributions from the excited-state corrections $\delta_{\mu}(2,3)$ is an incorrect approach to avoid violation of the size extensivity of the CR-EOMCC $(2,3)$ approaches and other analogous methods. The question proposed by our group was: how the computer code may be edited so that one removes the size extensive errors but in a more logical and analytical approach?

This code is available in so-called CC_PACKAGE, and can be modified to remove the size extensivity errors. The analysis of changes caused by modifying CC_PACKAGE code is done for previously studied nonineracting $\mathrm{C}_{2}+\mathrm{nNe}(\mathrm{n}=1,2,3)$ systems, where the $\mathrm{C}-\mathrm{C}$ bond distance was set up to be 2.348 bohrs and the modified aug-cc-pVDZ ${ }^{116}$ basis set was utilized. Furthermore, the performance of EOM-CCSD $(2)_{T}$ approach was tested by recalculating vertical excitation energies of $\mathrm{CH}^{+}, \mathrm{C}_{2}$ and $\mathrm{H}_{2} \mathrm{CO}$ molecules by our $\mathrm{H}-\mathrm{CR}$ $\operatorname{EOMCC}(2,3), A$ and $H-C R-E O M C C(2,3), D$. Note that the D variant differ from the A variant only in the form of the perturbative denominator, which in case of the former is of the Epstein-Nesbet type and for A it is the Möller-Plesset type. Also the CR-EOMCCSD(T),ID was modified in order to verify how the CR-EOMCCSD(T),ID approach would perform if corrected using Hirata's idea. ${ }^{115}$ These results were compared with $\operatorname{CR}-\operatorname{EOMCC}(2,3)$ variants ( $A$ and $D), C R-E O M C C S D(T), I D / I A, E O M-C C S D$ and $F C l$ values. In addition the relationship between Reduced Excitation Level (REL) and difference between CR$\operatorname{EOMCC}(2,3), A, C R-E O M C C(2,3), D$ and EOM-CCSD were examined as well as the accuracy of these methods compare to $\mathrm{FCl}$ for $\mathrm{C}_{2}, \mathrm{~N}_{2}$ and $\mathrm{H}_{2} \mathrm{O}$ molecules. 


\subsection{Results and Discussion}

Theaccuracy of the predicted vertical excitation energies with methods mentioned earlier is given as the error relative to $\mathrm{FCl}$ values, reported for $\mathrm{C}_{2}$ molecule by Christiansen et al. ${ }^{116}$ (see Table 3.1). In the case of neon atom(s) the aug-cc-pVDZ ${ }^{117}$ basis set was employed, the distance between $\mathrm{C}_{2}$ molecule and $\mathrm{Ne}$ atom(s), in the $\mathrm{C}_{2}+\mathrm{nNe}$ systems, was imposed to be $1000 \AA$ to ensure no interactions between these systems. All cal culations were preformed using the GAMESS ${ }^{118,119}$ software package, unless stated differently. Results presented in the Table 3.1 show that despite the size intensivity of $\mathrm{H}-\mathrm{CR}-\operatorname{EOMCC}(2,3), \mathrm{A}$ and $\mathrm{H}-\mathrm{CR}-\mathrm{EOMCC}(2,3)$,D the differences between results obtained with these approaches and $\mathrm{FCl}$ val ues are significant. For example, for ${ }^{1} \Pi_{\mathrm{u}}$ state of $\mathrm{C}_{2}+\mathrm{nNe}$ systems computed with the H-CR-EOMCC(2,3),A approach the excitation energies differ by $-17.3 \mathrm{eV}$. While even A variant of the size extensive $\operatorname{CR}-\operatorname{EOMCC}(2,3)$, which is generally less accurate than $\mathrm{D}$ variant, ${ }^{82}$ gives results that vary only $7.1 \mathrm{eV}$ from $\mathrm{FCl}$ for the $\mathrm{C}_{2}+\mathrm{Ne}$ (smallest) and $9.7 \mathrm{eV}$ for the $\mathrm{C}_{2}+3 \mathrm{Ne}$ (biggest) system. Moreover, the H-CR-EOMCC(2,3),D does not improve on values of excitation energies, as the difference is $-26.7 \mathrm{eV}$ compare to $\mathrm{FCl}$. With CR-EOMCC(2,3),D val ues of excitation energies of all states are improved, in case of ${ }^{1} \Pi_{u}$ state these differences are reduced to $3.9 \mathrm{eV}$ for the small lest and $9.7 \mathrm{eV}$ for the biggest of $\mathrm{C}_{2}+\mathrm{nNe}$ systems.

Next, we compared the size extensivity errors introduced by the methods considered here (see Table 3.2); these are the excitation energy differences $\left(\Delta \omega_{\mu}^{X}\right)$, defined as follows:

$$
\Delta \omega_{\mu}^{\mathrm{X}} \equiv \omega_{\mu}^{\mathrm{X}}\left(\mathrm{C}_{2}+\mathrm{nNe}\right)-\omega_{\mu}^{\mathrm{X}}\left(\mathrm{C}_{2}\right)
$$

where $\mathrm{n}$ is the corresponding number of $\mathrm{Ne}$ atoms added (one at the time) to the $\mathrm{C}_{2}$ molecule and $X$ is a given method employed to compute vertical excitation energies, e.g. $\mathrm{X}=\mathrm{CR}-\mathrm{EOMCC}(2,3), \mathrm{D}$. 
Table 3.1: Determination of accuracy of the CR-EOMCC, H-CR-EOMCC and EOMCCSD approaches with respect to $\mathrm{FCl}$ (in eV).

\begin{tabular}{|c|c|c|c|c|}
\hline \multirow[b]{2}{*}{ Method } & \multirow[b]{2}{*}{ State of $\mathrm{C}_{2}$} & \multicolumn{3}{|c|}{ Error relative to $\mathrm{FCl}$} \\
\hline & & $\mathrm{C}_{2}+\mathrm{Ne}$ & $\mathrm{C}_{2}+2 \mathrm{Ne}$ & $\mathrm{C}_{2}+3 \mathrm{Ne}$ \\
\hline $\mathrm{FCl}$ & & 0.000 & 0.000 & 0.000 \\
\hline EOMCCSD & & 3.282 & 3.282 & 3.282 \\
\hline CR-EOMCC(2,3),A & & 7.068 & 9.861 & 12.654 \\
\hline CR-EOMCC(2,3),D & & 3.907 & 7.031 & 9.677 \\
\hline aH-CR-EOMCC(2,3),A & $1^{1} \Pi_{u}$ & -17.297 & -17.297 & -17.297 \\
\hline H-CR-EOMCC(2,3),D & & -26.668 & -26.668 & -26.668 \\
\hline CR-EOMCCSD(T),ID/IA & & -3.222 & -1.348 & 0.379 \\
\hline H-CR-EOMCCSD(T),ID & & -19.355 & -18.694 & -18.069 \\
\hline $\mathrm{FCl}$ & & 0.000 & 0.000 & 0.000 \\
\hline EOMCCSD & & 108.863 & 108.863 & 108.863 \\
\hline CR-EOMCC(2,3),A & & 67.373 & 70.129 & 72.922 \\
\hline CR-EOMCC(2,3),D & & 50.689 & 53.776 & 56.459 \\
\hline aH-CR-EOMCC(2,3),A & $1^{1} \Delta_{\mathrm{g}}$ & 43.008 & 43.008 & 43.008 \\
\hline H-CR-EOMCC(2,3),D & & 20.077 & 20.077 & 20.077 \\
\hline CR-EOMCCSD(T),ID/IA & & 41.061 & 42.384 & 43.633 \\
\hline H-CR-EOMCCSD(T),ID & & 24.928 & 25.075 & 25.185 \\
\hline $\mathrm{FCl}$ & & 0.000 & 0.000 & 0.000 \\
\hline EOMCCSD & & 162.223 & 162.223 & 162.223 \\
\hline CR-EOMCC(2,3),A & & 163.399 & 166.192 & 168.985 \\
\hline CR-EOMCC(2,3),D & & 159.541 & 162.664 & 165.310 \\
\hline aH-CR-EOMCC(2,3),A & $1{ }^{1} \Sigma_{\mathrm{u}}^{+}$ & 139.034 & 139.034 & 139.034 \\
\hline H-CR-EOMCC(2,3),D & & 128.965 & 128.965 & 128.965 \\
\hline CR-EOMCCSD(T),ID/IA & & 153.587 & 155.498 & 157.299 \\
\hline H-CR-EOMCCSD(T),ID & & 137.454 & 138.189 & 138.851 \\
\hline $\mathrm{FCl}$ & & 0.000 & 0.000 & 0.000 \\
\hline EOMCCSD & & 177.033 & 177.033 & 177.033 \\
\hline CR-EOMCC(2,3),A & & 139.806 & 142.599 & 145.355 \\
\hline CR-EOMCC(2,3),D & & 123.195 & 126.319 & 129.002 \\
\hline aH-CR-EOMCC $(2,3), \mathrm{A}$ & $1^{1} \Pi_{g}$ & 115.441 & 115.441 & 115.441 \\
\hline H-CR-EOMCC(2,3),D & & 92.620 & 92.620 & 92.620 \\
\hline CR-EOMCCSD(T),ID/IA & & 112.538 & 113.861 & 115.147 \\
\hline H-CR-EOMCCSD(T),ID & & 96.405 & 96.552 & 96.699 \\
\hline
\end{tabular}

a Cal culated results equival ent to Hirata's method ${ }^{115}$ 
Table 3.2: Size extesivity errors (in eV) for the vertical excitation energies obtained with the CR-EOMCC, H-CR-EOMCC, and EOMCCSD approaches.

\begin{tabular}{|c|c|c|c|c|}
\hline \multirow[b]{2}{*}{ Method } & \multirow[b]{2}{*}{ State of $\mathrm{C}_{2}$} & \multicolumn{3}{|c|}{$\Delta \omega_{\mu}^{x^{b}}$} \\
\hline & & $\mathrm{C}_{2}+\mathrm{Ne}$ & $\mathrm{C}_{2}+2 \mathrm{Ne}$ & $\mathrm{C}_{2}+3 \mathrm{Ne}$ \\
\hline $\mathrm{FCl}$ & & 0.0 & 0.0 & 0.0 \\
\hline EOMCCSD & & 0.0 & 0.0 & 0.0 \\
\hline CR-EOMCC(2,3),A & & 2.793 & 5.586 & 8.379 \\
\hline CR-EOMCC (2,3),D & & 3.087 & 6.211 & 8.857 \\
\hline aH-CR-EOMCC $(2,3), \mathrm{A}$ & $1^{1} \Pi_{u}$ & 0.0 & 0.0 & 0.0 \\
\hline H-CR-EOMCC(2,3),D & & 0.0 & 0.0 & 0.0 \\
\hline CR-EOMCCSD(T),ID/IA & & 1.948 & 3.822 & 5.549 \\
\hline H-CR-EOMCCSD(T),ID & & 0.698 & 1.360 & 1.984 \\
\hline $\mathrm{FCl}$ & & 0.0 & 0.0 & 0.0 \\
\hline EOMCCSD & & 0.0 & 0.0 & 0.0 \\
\hline CR-EOMCC(2,3),A & & 2.793 & 5.549 & 8.342 \\
\hline CR-EOMCC(2,3),D & & 3.124 & 6.211 & 8.893 \\
\hline a H-CR-EOMCC(2,3),A & $1^{1} \Delta_{\mathrm{g}}$ & 0.0 & 0.0 & 0.0 \\
\hline H-CR-EOMCC(2,3),D & & 0.0 & 0.0 & 0.0 \\
\hline CR-EOMCCSD(T),ID/IA & & 1.360 & 2.683 & 3.932 \\
\hline H-CR-EOMCCSD(T),ID & & 0.110 & 0.257 & 0.367 \\
\hline $\mathrm{FCl}$ & & 0.0 & 0.0 & 0.0 \\
\hline EOMCCSD & & 0.0 & 0.0 & 0.0 \\
\hline CR-EOMCC(2,3),A & & 2.793 & 5.586 & 8.379 \\
\hline CR-EOMCC(2,3),D & & 3.124 & 6.247 & 8.893 \\
\hline aH-CR-EOMCC(2,3),A & $1^{1} \Sigma_{u}^{+}$ & 0.0 & 0.0 & 0.0 \\
\hline H-CR-EOMCC(2,3),D & & 0.0 & 0.0 & 0.0 \\
\hline CR-EOMCCSD(T),ID/IA & & 2.021 & 3.932 & 5.733 \\
\hline CR-EOMCCSD $(T), I D / I B$ & & 2.021 & 3.932 & 5.733 \\
\hline H-CR-EOMCCSD(T),ID & & 0.772 & 1.507 & 2.168 \\
\hline $\mathrm{FCl}$ & & 0.0 & 0.0 & 0.0 \\
\hline EOMCCSD & & 0.0 & 0.0 & 0.0 \\
\hline CR-EOMCC(2,3),A & & 2.793 & 5.586 & 8.342 \\
\hline CR-EOMCC(2,3),D & & 3.087 & 6.211 & 8.893 \\
\hline aH-CR-EOMCC (2,3),A & $1^{1} \Pi_{g}$ & 0.0 & 0.0 & 0.0 \\
\hline H-CR-EOMCC(2,3),D & & 0.0 & 0.0 & 0.0 \\
\hline CR-EOMCCSD(T),ID/IA & & 1.396 & 2.719 & 4.006 \\
\hline CR-EOMCCSD $(T), I D / I B$ & & 1.396 & 2.719 & 3.969 \\
\hline H-CR-EOMCCSD(T),ID & & 0.147 & 0.294 & 0.441 \\
\hline
\end{tabular}

${ }^{\text {a }}$ Cal culated results equival ent to Hirata's method ${ }^{115}$

b Given by Eq. (3.5) 
Table 3.2 shows that the H-CR-EOMCC(2,3),A approach do not introduce the size extensivity errors. This approach produce result equival ent to Hirata's method. ${ }^{115}$ Both A and $\mathrm{D}$ variants of $\mathrm{H}-\mathrm{CR}-\operatorname{EOMCC}(2,3)$ are modifications of the $\mathrm{CR}$-EOMCC $(2,3)$ method obtained by removing the ground state corrections from $\delta_{\mu}(2,3)$. However, small sizeextensivity errors are introduced with $\mathrm{H}-\mathrm{CR}-\mathrm{EOMCCSD}(\mathrm{T}), \mathrm{ID}$ approach. Furthermore, $\mathrm{H}$ CR-EOMCCSD(T),ID vertical excitation energies values are al so less accurate than those obtained via the CR-EOM CCSD(T) method (see Table 3.1).

Before discussing the role of the deexcitation operator $L_{\mu, 3}$, defined by amplitudes $\underset{\mu, i j k}{a b c}$, with respect to the size extensivity error of excitation val ues obtained with $\operatorname{CR}-\operatorname{EOMCC}(2,3)$ method, results for excited state energies of $\mathrm{C}_{2}, \mathrm{~N}_{2}$ and $\mathrm{H}_{2} \mathrm{O}$ (Table 3.3) and vertical excitation energies of $\mathrm{CH}^{+}, \mathrm{H}_{2} \mathrm{CO}, \mathrm{C}_{2}, \mathrm{~N}_{2}$ and $\mathrm{H}_{2} \mathrm{O}$ (Table 3.4 and Table 3.5) will be discussed. Structural parameters for these molecular systems are:

† for $\mathrm{CH}^{+}$the bond length $(r)$ is $1.131 \AA$;

† for $\mathrm{H}_{2} \mathrm{CO}: \mathrm{r}_{\mathrm{CO}}=1.208 \AA, \mathrm{r}_{\mathrm{CH}}=1.116 \AA$ and $\mathrm{H}-\mathrm{C}-\mathrm{H}$ bond angle is $116.5^{\circ}$ at $298 \mathrm{~K}$

† for $\mathrm{N}_{2}: \mathrm{r}=2.068 \mathrm{Bohr}$

† for $\mathrm{C}_{2}: \mathrm{r}=2.348 \mathrm{Bohr}$

In the case of $\mathrm{H}_{2} \mathrm{O}$ we used $\mathrm{C}_{2 v}$ symmetry and cartesian coordinates: $\mathrm{O}(0,0,0)$ and $\mathrm{H}(0, \pm 1.429937284,-1.107175113)$, in atomic units. The following basis sets were employed for $\mathrm{CH}^{+}$, formal dehyde, $\mathrm{N}_{2}$, and $\mathrm{C}_{2}$ respectively: aug-cc-pVDZ (ACCD) and augcc-pVTZ (ACCT), ACCT and modified aug-cc-pVDZ ${ }^{116}$ (mACCD), cc-pVDZ (CCDZ), and mACCD. $\mathrm{FCl}$ serves again as our benchmark. For $\mathrm{CH}^{+}, \mathrm{H}_{2} \mathrm{CO}$, and $\mathrm{C}_{2}$ values obtained with this method, are taken from the Hirata's work; ${ }^{115}$ for $\mathrm{N}_{2}$ and $\mathrm{H}_{2} \mathrm{O}$ results refer to Christiansen et al. ${ }^{116}$ 
Results for the excited-state energies presented in Table 3.3 show that all val ues produced with variant $D$ of the $C R-\operatorname{EOMCC}(2,3)$ approach are more accurate than those obtained with variant $A$, except for the ${ }^{1} \Sigma_{u}^{+}$state of $C_{2}$ molecule. For example, the error (i.e., difference between a given excited state energy obtained with $\mathrm{FCl}$ and corresponding value predicted with an approximated method) in the $\Pi_{u}$ state of $C_{2}$ molecule is reduced by $9.4 \mathrm{mHa}$ (millihartrees). This error for both $\mathrm{CR}$ - $\operatorname{EOMCC}(2,3)$ variants is much smaller than the one obtained with the EOMCCSD method ( $33 \mathrm{mHa})$. A similar improvement is also seen for other states and molecules; for instance, in the ${ }^{1} \Sigma_{u}^{-}$state of $N_{2}$ molecule the error at EOMCCSD level is reduced from $18.5 \mathrm{mHa}$ to 6.6 and $1.4 \mathrm{mHa}$ in the CR$\operatorname{EOMCC}(2,3), \mathrm{A}$ and the CR-EOMCC(2,3),D, respectively.

Table 3.4 and Table 3.5 al so include Relative Excitation Levels (REL), which will allow us to compare the performance of a given method with respect to exited-states dominated by one or two electron excitations. Results for the vertical excitation energies show that for excited-states dominated by two electron excitations $\left({ }^{1} \Delta_{\mathrm{g}},{ }^{1} \Pi_{\mathrm{g}}\right.$ of $\mathrm{C}_{2}$ and ${ }^{1} \Delta,{ }^{1} \Sigma^{+}$of $\left.\mathrm{CH}^{+}\right)$ the CR-EOMCC(2,3),D approach performed better than CR-EOMCC(2,3),A, i.e., obtained values are lower, yet above $\mathrm{FCl}$. In the case of singly excited states CR-EOMCC(2,3),D performs either better or equival ently well as variant $A$ of this approach. For all states of molecules examined here, except of ${ }^{1} \mathrm{~A}_{2}$ in $\mathrm{H}_{2} \mathrm{CO}$ and ${ }^{1} \mathrm{\Pi}_{\mathrm{g}}$ in $\mathrm{N}_{2}$, both variants of the CR-EOMCC $(2,3)$ approach predicted vertical excitation energy val ues more accurately than EOMCCSD. Hirata's EOM-CCSD $(2) T^{115}$ method, reproduced here with the H-CREOMCC(2,3),A approach, performs better than EOMCCSD; however for most of the excited states predicted with both variants of this method vertical excitation energy val ues are below $\mathrm{FCl}$, which is unacceptable. 


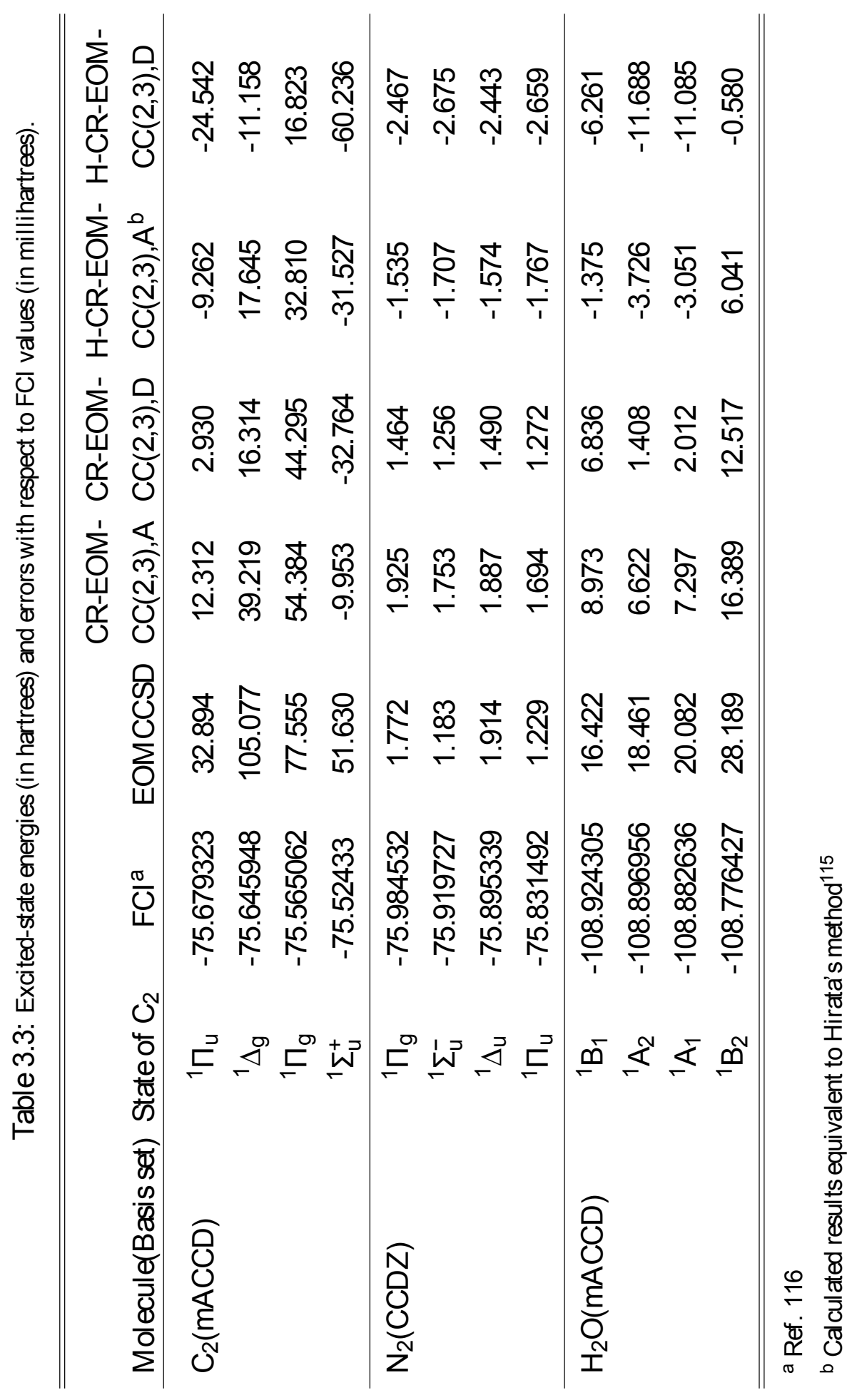




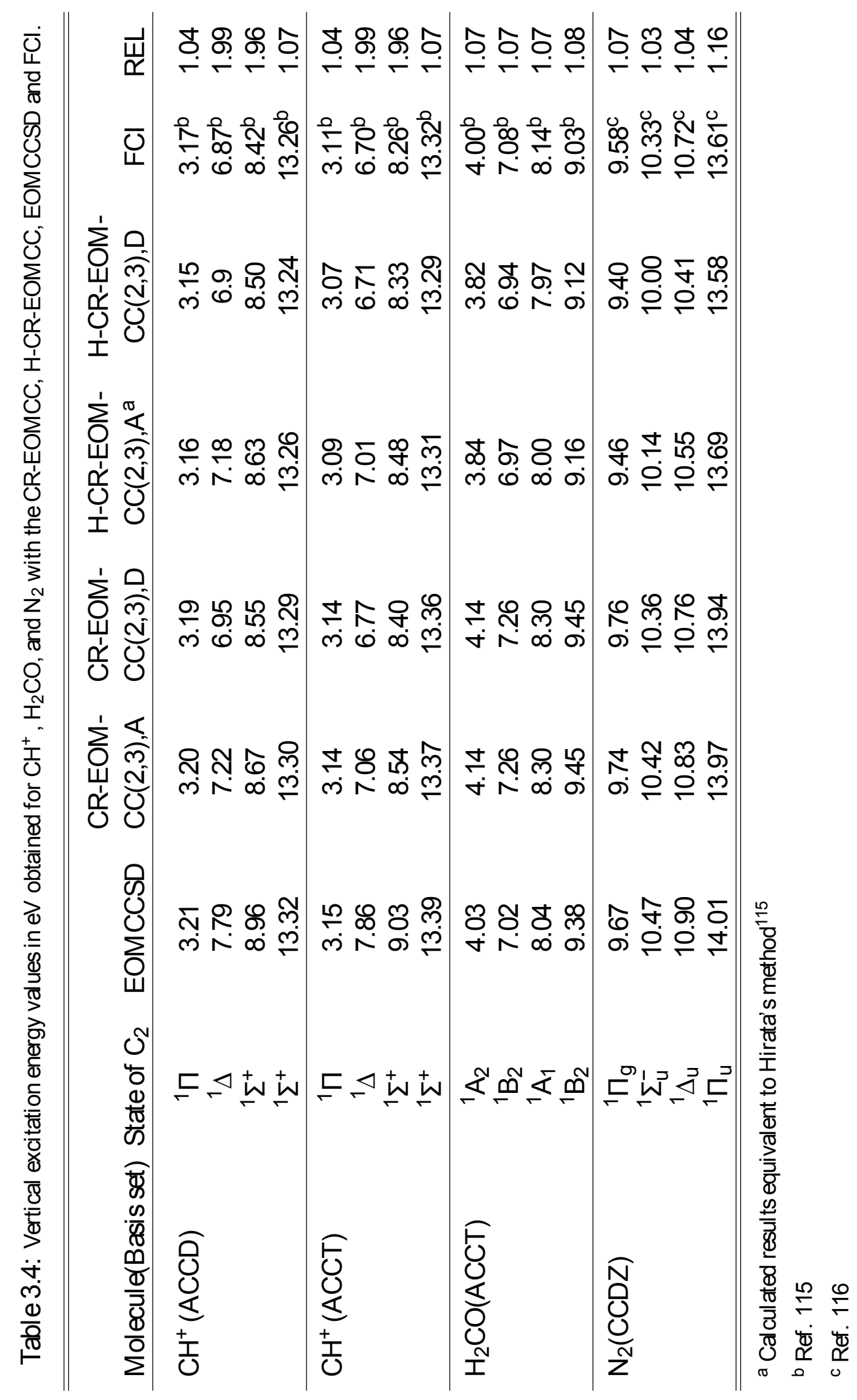




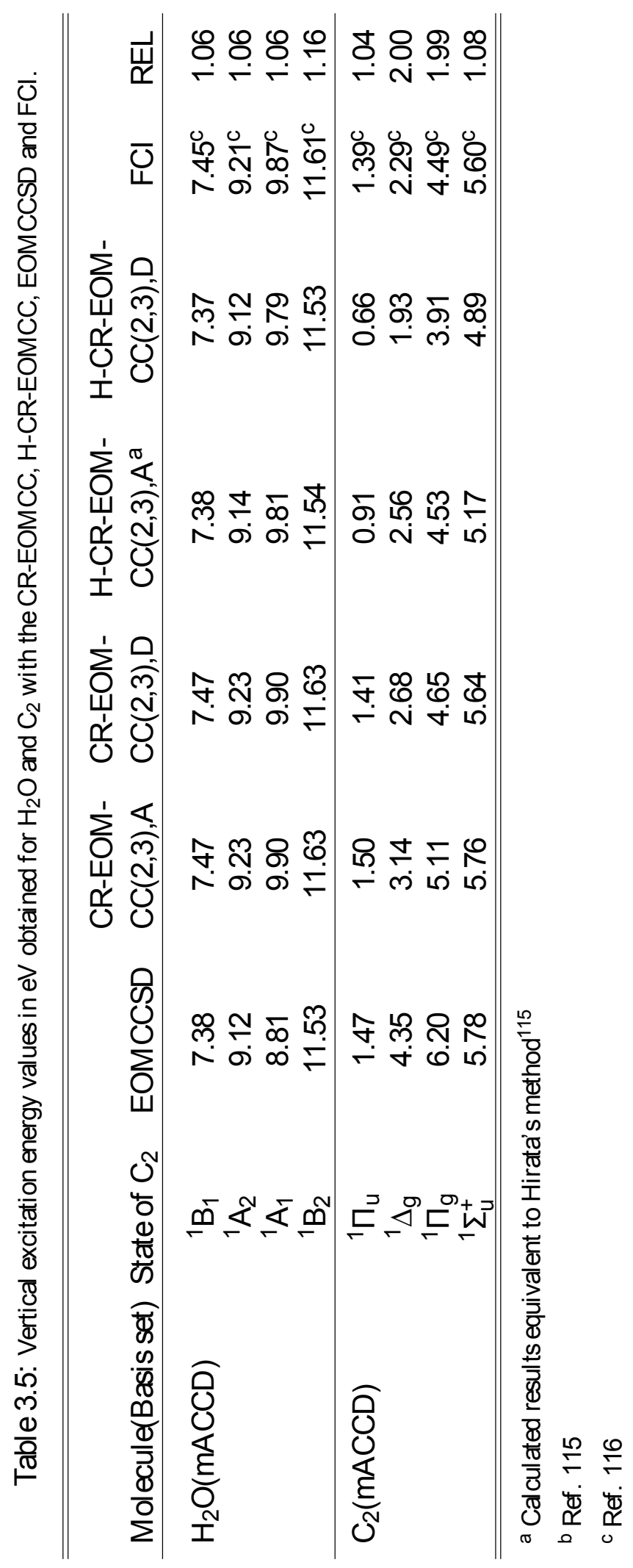


The most severe examples are resultsfor $\mathrm{C}_{2}$ obtai ned with $\mathrm{D}$ variant of $\mathrm{H}-\mathrm{CR}-\mathrm{EOMCC}(2,3)$ (i.e., a modified version of the best $\operatorname{CR}-\operatorname{EOMCC}(2,3)$ model using Hirata's idea); in fact for all the states the vertical excitation energies are less than the corresponding $\mathrm{FCl}$ values. Furthermore differences are about $-0.7 \mathrm{eV}$ for the excited-states when REL is about 2 and between $-0.4 \mathrm{eV}$ to $-0.6 \mathrm{eV}$ for those excited-states with REL close to 1 . This clearly shows that although EOM-CCSD $(2)_{T}$ does not introduce size extensivity error, which only slightly worsens vertical excitation energies, when values cal culated with CR$\operatorname{EOMCC}(2,3), \mathrm{A}$ and CR-EOMCC(2,3),D are compared with H-CR-EOMCC(2,3),A and H-CR-EOMCC(2,3),D, respectively the modified methods produce worse results. Other sources of errors ${ }^{70}$ have more significant effect on results as they might be not only inaccurate but also artificial. Also, it is worth to mention that for doubly excited states the H-CR-EOMCC(2,3),A approach does not reduce errors in values of vertical excitation energies obtained with EOMCCSD as effectively as the CR-EOMCC(2,3),D approach. For instance, the EOMCCSD error with respect to $\mathrm{FCl}$ for ${ }^{1} \Delta$ state in $\mathrm{CH}^{+}$(ACCD) calculations is $0.92 \mathrm{eV}$ which decreases to $0.08 \mathrm{eV}$ with $\mathrm{CR}$-EOMCC(2,3),D and to $0.31 \mathrm{eV}$ with H-CR-EOMCC(2,3),A.

\subsection{Insights into the Role of ${ }_{\mu}$ in the Size Extensivity Er- ror}

As indicated earlier the problem of size extensivity of the approximated CR-EOMCC approaches and related methods was analyzed before by Piecuch et al..$^{70,82}$ and similar conclusions were drawn. However these studies focused only on the role of $r_{\mu, 0}$ in $\mathrm{C}_{2}+\mathrm{nNe}$ systems, whereas our group analyzed al so properties of $\mu$ in the attempt of finding a way to restore size intensivity of $\omega_{\mu}^{\mathrm{CR}-\operatorname{EOMCC}(2,3)}$. This aspect represents the novelty of our work. 
Triples corrections $\delta_{\mu}(2,3)$ to the excitation energy $\omega_{\mu}^{\text {CR- EOMCC }(2,3)}$ defined in Eq. (3.2) can be written as follows:

$$
\delta_{\mu}(2,3) \equiv\left\langle\Phi\left|L_{\mu, 3} M_{\mu, 3}(2)\right| \Phi\right\rangle=\sum_{\substack{i<j<k \\ a<b<c}} \underset{\mu, i j k}{a b c} M_{\mu, a b c}^{i j k}(2) .
$$

Being $\omega_{\mu}^{\mathrm{CR}-\operatorname{EOMCC}(2,3)}$ the energy difference, $\delta_{\mu}(2,3)$ can be written as

$$
\delta_{\mu}(2,3)=\sum_{\substack{i<j<k \\ a<b<c}}\left(M_{\mu, a b c}^{i j k}-\underset{0, i j k}{a b c} M_{0, a b c}^{i j k}\right)
$$

Eq. (3.7) can be further rearranged in terms of linear excitation operators $\left(R_{\mu, 0}, R_{\mu, 1}, R_{\mu, 2}\right)$ giving rise to the following set of equations:

$$
\begin{aligned}
& \delta_{\mu}(2,3)=\sum_{\substack{i<j<k \\
a<b<c}}^{a b c}{ }_{\mu, i j k}^{a b}\left[M_{\mu, a b c}^{i j k}\left(R_{\mu, 0}\right)+M_{\mu, a b c}^{i j k}\left(R_{\mu, 1}+R_{\mu, 2}\right)\right]-\sum_{\substack{i<j<k \\
a<b<c}}^{a b c}{ }_{0, i j k} M_{0, a b c}^{i j k} \\
& \delta_{\mu}(2,3)=\sum_{\substack{i<j<k \\
a<b<c}}^{a b c}{ }_{\mu, i j k}^{a b}\left[r_{\mu, 0} M_{\mu, a b c}^{i j k}+M_{\mu, a b c}^{i j k}\left(R_{1}+R_{2}\right)\right]-\sum_{\substack{i<j<k \\
a<b<c}}{ }_{0, i j k}^{a b c} M_{0, a b c}^{i j k} \\
& \delta_{\mu}(2,3)=\sum_{\substack{i<j<k \\
a<b<c}}\left[\left(r_{\mu, 0}^{a b c} \underset{\mu, i j k}{a b}-\underset{0, i j k}{a b c}\right) M_{0, a b c}^{i j k}+M_{\mu, a b c}^{i j k}\left(R_{1}+R_{2}\right)\right]
\end{aligned}
$$

This last equation needs to be rewritten in order to obtain working equations leading to efficient fully vectorized computer code (for more details see work by Wloch et al. ${ }^{70}$ ). Nevertheless it shows al ready that not only the $r_{\mu, 0}$ term but also $\mu$ can be the source of the size extensive error of $\omega_{\mu}^{\mathrm{CR}-\operatorname{EOMCC}(2,3)}$.

To test the influence of $r_{\mu, 0}$ on properties of $\mu$ a part of the code (viz. "Loop for $\mu$ ") was implemented to cal culate triples corrections $\delta_{\mu}(2,3)$ of $\operatorname{CR}-\operatorname{EOMCC}(2,3)$ method (available in the CC_PACKAGE). Interestingly, if $r_{\mu, 0}$ was initially zero, whether or not this part of the code was commented in or out, it did not change results when the value of $r_{\mu, 0}$ was explicitly changed. However if $r_{\mu, 0}$ was not initially zero then the excitation results would change accordingly with this part of the code commented in (i.e., excluded). The 
set of $\mu$ was not zero, but it was the same for all the states in which $r_{\mu, 0}$ was initially null. Nevertheless it did change when $r_{\mu, 0}$ was not initially zero.

As a final point it is important to mention that in the case of $\operatorname{CR}-C C(2,3) r_{\mu=0,0}=1$ (where 1 is the unit operator), $r_{\mu, 0} \approx 0$, and $I_{\mu=0,0}$ but does not equal to $I_{\mu, 0}$. Moreover in the situation when reference value of $I_{\mu}$ is zero (i.e., different symmetry of the excited-state $\mu$ than the ground-state) the contributions from the excitation operator $R_{\mu, 0}$ vanish from $\delta_{\mu}(2,3)$. This leads to less corrected excited state energies (red dotted curve in Fig. 3.1) in comparison to corresponding correction for the ground-state energies (blue dotted curve in Fig. 3.1), and greater $\omega_{\mu}^{\mathrm{CR}-\operatorname{EOMCC}(2,3)}$ value. One of the explicit values for $r_{\mu, 0}$ was 1 $\left(r_{\mu, 0}=1\right)$ and the expectation was this will enforce the recovery of the "missing" part of $\delta_{\mu}(2,3)$ correction (green dashed curve in Fig. 3.1).

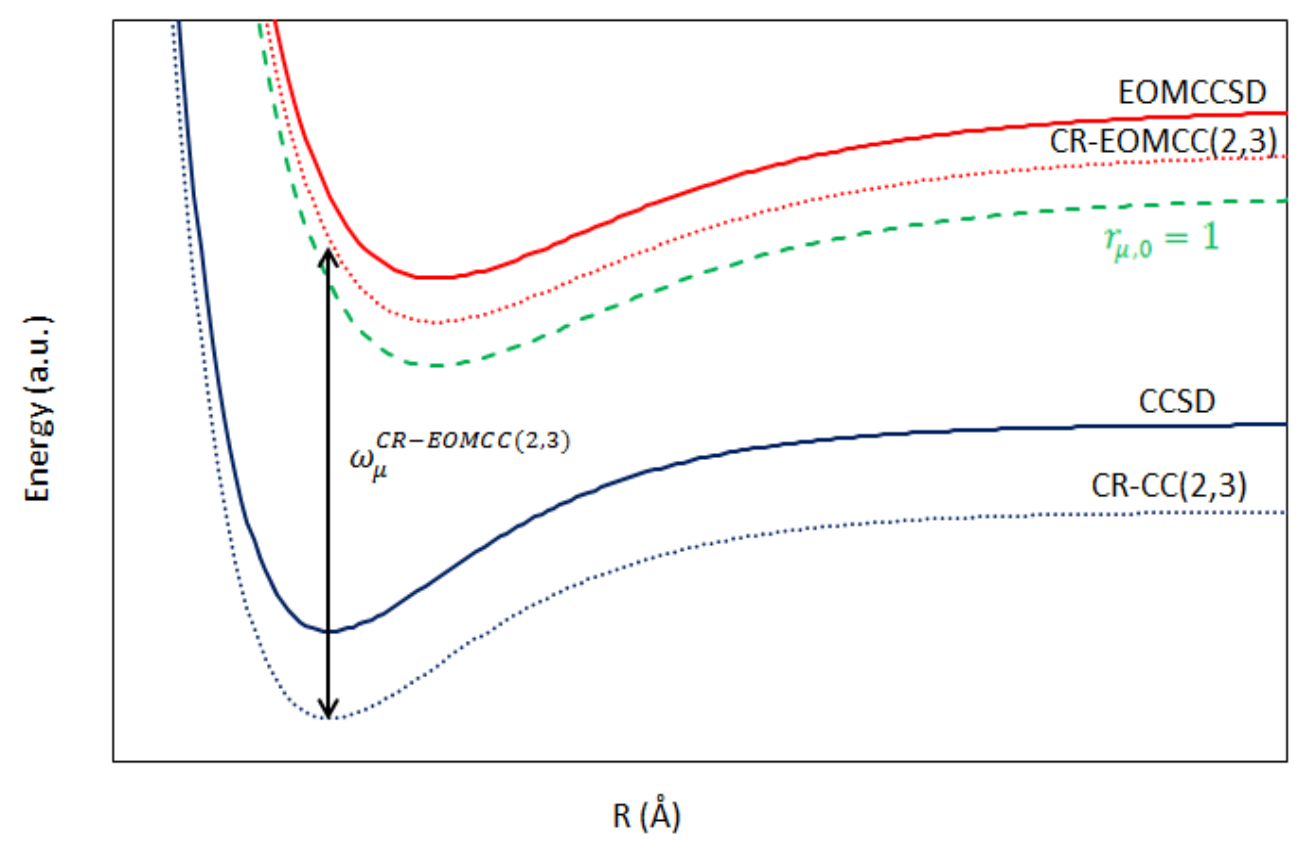

Figure 3.1: Hypothetical Morse curves for the ground- and excited-state. 


\subsection{Conclusions}

Hiratas EOM-CCSD(2)T seems to improve the results for doubly excited states but at the same time the excitation energy values for singly excited states are much lower than $\mathrm{FCl}$ values. Hirata simply removes completely the ground state correction; this may produce an error much larger than that arising from the size extensivity error. Hiratas EOM-CCSD(2)T is indeed size extensive but it loses in rigor and accuracy. At the same time the earlier developed H-CR-EOMCCSD(T),ID approach cannot be expected to become size extensive by removing the ground state correction because this method is not such even for the ground-state. Therefore $\mathrm{CR}-\mathrm{CCSD}(\mathrm{T})$ should be replaced by the newer and more robust CR-CC $(2,3)$ method which does not introduce size extensivity error. From tests on $\mathrm{C}_{2}+\mathrm{nNe}$ systems it was shown that the size-extensivity is indeed due to a vanishing correction for the ground state. ${ }^{82}$ In other words the value of this correction did not change when excited states for $\mathrm{C}_{2}, \mathrm{C}_{2}+\mathrm{Ne}$ or $\mathrm{C}_{2}+2 \mathrm{Ne}$ were cal culated. This is because in the ground state $R_{0,0}=1$ whereas in the excited state $R_{\mu, 0}$ is usually close to 0 . Hence by forcing a priori the right operator to be equal to 1 (i.e., for ground and/or excited states) seemed to be a better procedure to remove the size extensivity error. This would be the case if not for the fact that in the formulation of $\operatorname{CC}-\operatorname{EOMCC}(2,3)$ the left deexcitation operator $\left(L_{\mu, 3}\right)$ is multiplied by $R_{0}$ where the former is contributing to this error. Furthermore the amplitudes $\underset{\mu, \mathrm{ijk}}{\mathrm{abc}}$ are extremely small for excited states for which $\mathrm{R}_{\mu, 0}$ was forced to be 1 . Therefore it seems that the remedy for removing the size extensivity error from CC-EOMCC $(2,3)$ lays in including the same left vector to the ground state as the one in the excited state (as right now $L_{0}^{(A)}=L_{\mu}^{A}$ which is due to use of Eq. (2.83)). 


\section{Chapter 4}

\section{UV-Vis Spectra of Copper (II) \\ Complexes with I midazole Derivatives \\ from Long-Range Cor rected Density \\ Functionals ${ }^{1}$}

Metal complexes with ligands containing imidazole ring play an important role in biological systems; for example, the imidazole-4-acetate (denoted as IA) is one of the receptors for GABA inhibitory neurotransmitter found in mammalian brains. ${ }^{122}$ Previous studies by Drożdżewski and coworkers ${ }^{123-125}$ were mainly focused on the experimental determination of the structural and spectroscopic properties of this complex as well as its hydrated analog. To the best of my knowledge the only theoretical study was preformed by Drożdżewski and Pawlak ${ }^{125}$ utilizing B3LYP functional and LanL2DZ basis set on Cu atom and d-polarization on $\mathrm{N}$ and $\mathrm{O}$ atoms. They computed structural parameters and the

${ }^{1}$ Part of the material contained in this Chapter has been adapted from accepted for publication article. ${ }^{120}$ Another part will be used for publication in collaboration with Prof. Bryant (see Preface). ${ }^{121}$ 
IR spectrum of the $\left[\mathrm{Cu}(\mathrm{IA})_{2}\right]$ complex. A derivative of this complex was prepared by the Chavez group, ${ }^{120}$ where the $\mathrm{H}$ atom of the $1 \mathrm{H}$-imidazole-4-acetate is substituted with an ethyl group forming the 1-ethyl-1H-imidazol-4ylacetate. The bis(2-(1-ethyl-1H-imidazol4-yl)acetate)copper(II) complex (denoted later in this text as [ $\left.\mathrm{Cu}(\mathrm{IAC})_{2}\right]$ ) is soluble in various organic solvents.

Apart from the importance in the central nerve system these complexes represent interesting case studies from a theoretical point of view due to their open-shell character. It is known (see, for example, work of Romaniello et al. ${ }^{126}$ ) that for open-shell ground-state systems doubly and higher excited states are important to properly describe their properties. This task is particularity challenging since TDDFT fails in providing reliable information for excited states dominated by multiple electron excitations. Furthermore, such limitations can al so be encountered by other theoretical methods. For instance, despite the CR-EOMCC $(2,3)$ method is able to produce accurate excited-state energies, especially for the excited-states dominated by two-electron excitations, it does not necessarily improve on the accuracy of excitation energies. This is due to lack of size extensivity of this property (Chapter 3). Moreover, most of the metal-organic complexes are simply too large to be studied computationally on a typical computer cluster at such high level of theory. Hence, despite its limitations, TDDFT is the chosen method given its timely and computationally inexpensive capability of modeling optical spectra. Obviously the choice of the exchangecorrelation (xc) functional approximation is a crucial step in obtaining meaningful results. In fact no "black box" DFT method exists able to perform properly, and adequately solve chemical problems of a different nature. To illustrate how difficult this step might be, afew related studies are described in this section.

In a theoretical study Peng and coworkers ${ }^{127}$ obtained a UV-Vis spectrum of the [ $\mathrm{Cu}(\mathrm{mal})(\mathrm{PIM})_{2} \mathrm{H}_{2} \mathrm{O}$ ] complex using the standard hybrid functional (B3LYP), and reported reasonable agreement with experimental data, i.e., with an error of $0.3 \mathrm{eV}$. On the other 
hand an earlier work of Sinnecker and Nesse ${ }^{128}$ showed that TD-B3LYP cal cul ations failed to reproduce absorbtion spectrum of a "blue" protein called plastocyanin, which also contains $\mathrm{Cu}(\mathrm{II})$ cation. Interestingly, even for ground-state properties, such as structure and IR/Raman spectra, the theoretical cal culations for copper(II) complexes seem to be difficult. Atanasov et al. ${ }^{129}$ suggested that no general density functional is currently able to accurately describe different types of ligands. Contrary to this statement, a later study of the $\mathrm{Cu}(\mathrm{II})-\mathrm{L}$ bonding of isatin-shiff base and related complexes showed that the BP86 functional with the TZVP basis set performed quite well. ${ }^{130}$ Moreover, both B3LYP and BP86 functionals are commonly used to obtain properties of other organometal lic systems. For example, the BP86 functional was employed in TDDFT cal culations to study the excitation energies of some transition metals involving the $d^{1}$ val ence configuration of $M L X_{4}$ systems. ${ }^{131}$ The conclusion of that work was that doublet-doublet transitions computed with BP86/ALDA were fairly accurate (i.e., within an error of about 0.3-0.5 eV). In contrast Wu et al., ${ }^{132}$ who also employed such functionals to predict UV photofragmentation of the $\left[\mathrm{Mn}(\text { pyridine })_{4}\right]^{2+}$ complex, concluded that electron excitations obtained via adiabatic TDDFT should not be used to quantitatively describe experimental data.

These few examples illustrate only the problem of choosing a density functional approximation but more challenges can be found in the literature. ${ }^{42}$ Despite some recent developments (such as range-separation, ${ }^{96,133}$ high level parametrization, e.g., Truhlar's group M06 family of functionals, ${ }^{94}$ double hybridization ${ }^{134}$ ) choosing a functional that would significantly improve upon hybrid GGA is still a big question. For instance, in recent study on singlet-triplet transition properties for 6-phenyl-2,2' -bypyridine tridentate iridium(III) complex, ${ }^{135}$ a closed-shell ground-state system, it was demonstrated that the PBE0 functional with LanL2DZ (on metal), and 6-31G(d) (on ligand) outperformed the long-range corrected (LRC) density functionals. Although, it is thought that employing LRC-TDDFT has an advantage over standard TDDFT in describing the long-range charge transfer (CT) transition of ligand-to-metal type that are present in the electronic spectrum of transition 
metal complexes. Nevertheless, it would be interesting to explore if standard hybrid functionals can perform better also in predicting transitions of open-shell transition metal complexes, such as those considered herein $\left(\left[\mathrm{Cu}(\mathrm{IA})_{2}\right]\right.$ and $\left.\left[\mathrm{Cu}(\mathrm{IAC})_{2}\right]\right)$. Finally, noteworthy issue is the effect of the range separation parameter $(\mathrm{Y})$ on the metal-centered $d-d$ transitions. To the best of my knowledge this work represents the first attempt to study the influence of $y$ on this kind of transitions.

The structural parameters of $\left[\mathrm{Cu}(\mathrm{IA})_{2}\right]$ were optimized with BP86 (pure GGA), B3LYP (hybrid GGA) and M06L (meta GGA) functionals. These density functional s were utilized with three popular small effective core pseudopotentials (ECPs) and their corresponding basis sets for $\mathrm{Cu}$ and 6-31G(d) basis set for $\mathrm{H}, \mathrm{N}, \mathrm{C}$ and $\mathrm{O}$ (ligand atoms). The following ECPs were employed: LanL2DZ/[3s3p2d], Stuttgart RSC/[6s5p3d] and cc-pVDZPP/[5s4p3d1f]. Next, after the best combination functional and ECP/basis set for Cu was determined, the 6-31G(d) basis set originally used on ligand atoms was sequentially increased to determine their effect on structural parameters. In other words, calculated cartesian coordinates from the best combination were reoptimized employing: 6-31G* * $6-31+G^{*}, 6-31+G^{* *}$ and $6-31++G^{* *}$ basis sets on the ligand atoms. The obtained bond lengths and angles were compared to experimental val ues. This step all owed us to evaluate the best functional with the best EPC and basis sets on the atoms. This level of theory was then utilized to compute structural parameters of $\left[\mathrm{Cu}(\mathrm{IAC})_{2}\right]$. This complex, as mentioned earlier, is solvable in acetonitrile (to some extent), DMF, methanol, chloroform and dichloromethane. Therefore, it was important to determine the effect of solvation on the structural parameters of $\left[\mathrm{Cu}(\mathrm{IAC})_{2}\right]$. This was archived via Integral Equation Formalism of The Polarizable Continuum Model (IEF-PCM). ${ }^{136,137}$ Methanol and dichloromethanewere chosen because they represent organic solvents of two different types. In fact, the former is characterized by a high dielectric constant $(\varepsilon=32.613)$ which can be expected to interact with the complex; the latter on the opposite has a low diel ectric constant $(\varepsilon=8.93)$ that is unlikely going to interact with the complex. After every geometry optimization frequency 
analysis was performed to ensure that computed structures represent the minimum of the potential energy surface. This transl ates into no negative frequencies for the normal modes.

In summary in order to obtain optimized geometries, the following sets of calculations were performed for obtaining geometries:

† First set. BP86, B3LYP, and M06L employed. For Cu the following ECPs with the corresponding basis sets: LanL2DZ/[3s3p2d], Stuttgart RSC/[6s5p3d], and cc-pVDZ-PP/[5s4p3d1f]. For ligand atoms 6-31G* basis sets was utilized.

† Second set. Using the coordinates in best agreement with experiments, the structural parameters of $\left[\mathrm{Cu}(\mathrm{IA})_{2}\right]$ are reoptimized with several different basis sets for ligand atoms: $6-31 G^{*}, 6-31 G^{* *}, 6-31+G^{*}, 6-31+G^{* *}$ and $6-31++G^{* *}$.

† Third set. Using the best combination the structural parameters of $\left[\mathrm{Cu}(\mathrm{IAC})_{2}\right]$ are computed in vacuum, methanol and dichloromethane.

The reason to perform the first set of cal culations is two-fold: in fact, it allows finding both the best ECP and basis set for $\mathrm{Cu}$, and it shows the effects of various density functionals on the bonds and angles. As mentioned before the second set is performed to establish the influence of the chosen basis set to describe ligand atoms. The third and last step, shows changes into the structures due to their interactions with surrounding environment such as different solvents.

Vertical excitation energies and oscillator strengths were computed employing TDDFT and LRC-TDDDF to describe absorption spectra of bis(imidazole-4-acetato)copper(II) and bis(2-(1-H-imidazole-4-yl)acetate)copper(II) complexes. Several long-range corrected exchange-correlation functionals ( $\omega B$ 97X, LC- $\omega P B E$, original CAM-B3LYP and CAMB3LYP* ) were investigated against commonly utilized standard xc functional s like BP86, 
B3LYP and M06L. Results are compared with experimental absorption bands to establish the reliability of these functionals. In addition, calculations with various values of the range-separation parameter $(\mathrm{Y})$ and using CAM-B3LYP* functional where performed to explore different $y$ val ues as recommended into the literature (see discussion below).

In the original CAM-B3LYP 103 the range separation is defined for $Y=0.33, \alpha=0.19$ and $\beta=0.46$. However the exact $-\frac{1}{r}$ asymptotic behaviour of the exchange potential cannot be properly restored unless $\alpha+\beta=1$. This issue is taken care of by changing $\beta=0.46$ to $\beta=0.81$; this variant of the functional is denoted as CAM-B3LYP* Similarly, the parameterized $\omega B 97 X^{138}$ employs a comparable fractions of HF exchange where $\alpha \approx 0.16, \beta \approx 0.84$, whereas LC- $\omega \mathrm{PBE}^{139,140}$ does not include any short-range HF exchange contribution, i.e., $\alpha=0$ and $\beta=1$. In addition, LC- $\omega P B E$ has the highest value of the range separation parameter $(y=0.40)$ when optimized for ground-state properties. Note that for excited-states a lower $\mathrm{y}$ for this functional is recommended, ${ }^{141}$ i.e., $\mathrm{Y}=0.20$. For $\omega B 97 X y=0.30$, while for LC- $\omega$ PBEh $y=0.20$ is optimized for both ground- and excited-states.

A brief overview of DFT and TD-DFT is given in Chapter 2, sections 2.1.3 and 2.1.3.1, and the LRC scheme is discussed in subsection 2.2.2.1.

Calculations were performed using either Gaussian $03^{142}$ or Gaussian $09,{ }^{143}$ depending on Density Functional Approximation (DFA) employed. The 03 version was employed for all the standard $x c$ functionals (except of M06L), while the 09 version was employed for all the LRC functionals. Based on the band assignments for the reflectance spectra of $\left[\mathrm{Cu}(\mathrm{IA})_{2}\right]$ complex $^{123}$ the expected types of el ectronic transitions are: Iigand-to-metal $\mathrm{CT}$ (LMCT), ligand-to-ligand (LL) and d-d on Cu(II) center a.k.a ligand-field (LF). This scenario implies the main challenge to describe metal - and ligand-based transitions with similar accuracy. Other possible computational challenges are represented by appearance of 
excited states carrying nonphysically high spin contamination, and mixed character of excited states. Consequently, a highly accurate quantitative agreement between experiment and theory cannot be expected. Instead, this work is focused on achieving two goals. First: to find the LRC functional that improve on the accuracy of excitation energies predicted with respect to standard $x c$ functionals. Second: to provide new insights into the understanding of the effect of the range-separation parameter and the fraction of exact exchange on electronic transitions. The analysis will be mainly done for $d-d$ transitions, based on results for $\left[\mathrm{Cu}(\mathrm{IA})_{2}\right]$ obtained with $C A M-B 3 L Y P^{*}$ using various values of $y$ and fraction of exact exchange. This set of calculations should provide the best amount of the exact exchange (a.k.a. HF exchange and denoted as $\alpha$ ). Although, since $y$ is system dependent, ${ }^{111}$ cal culations with various val ues of the range-separation parameter will have to be repeated for $\left[\mathrm{Cu}(\mathrm{IAC})_{2}\right]$ in order to check the applicability of our approach to this complex as well.

\subsection{DFT and TDDFT Results}

\subsubsection{Structural Parameters}

Before discussing the UV-Vis spectra of $\left[\mathrm{Cu}(\mathrm{IA})_{2}\right]$ and $\left[\mathrm{Cu}(\mathrm{IAC})_{2}\right]$ obtained with BP86 (pure GGA), B3LYP (hybrid GGA), and M06L (meta GGA), the process of choosing the best ECP and basis sets utilized to cal culate geometries will be presented. The molecular arrangement of these complexes is shown in Fig. 4.1 and Fig. 4.2.

The comparison between selected structural parameters obtained with various standard $x c$ functionals using LanL2DZ/[3s3p2d] (LL2DZ), Stuttgart RSC/[6s5p3d] (SRSC) and ccpVDZ-PP/[5s4p3d1f] (CCDPP) for Cu and 6-31G* for ligand atoms is shown in Table 4.1, Table 4.2 and Table 4.3, respectively. 


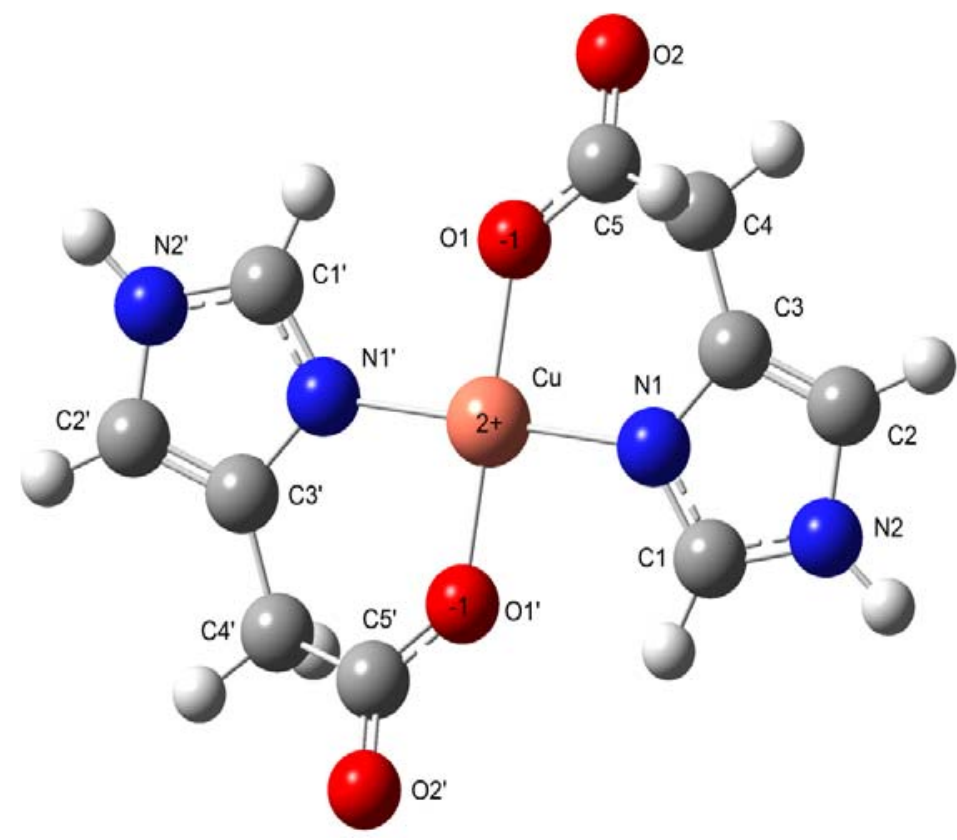

Figure 4.1: Molecular structure of the $\left[\mathrm{Cu}(\mathrm{IA})_{2}\right]$ complex. The following colors salmon, gray, red, and white, represent $\mathrm{Cu}, \mathrm{C}, \mathrm{O}, \mathrm{N}$, and $\mathrm{H}$ atoms, respectively.

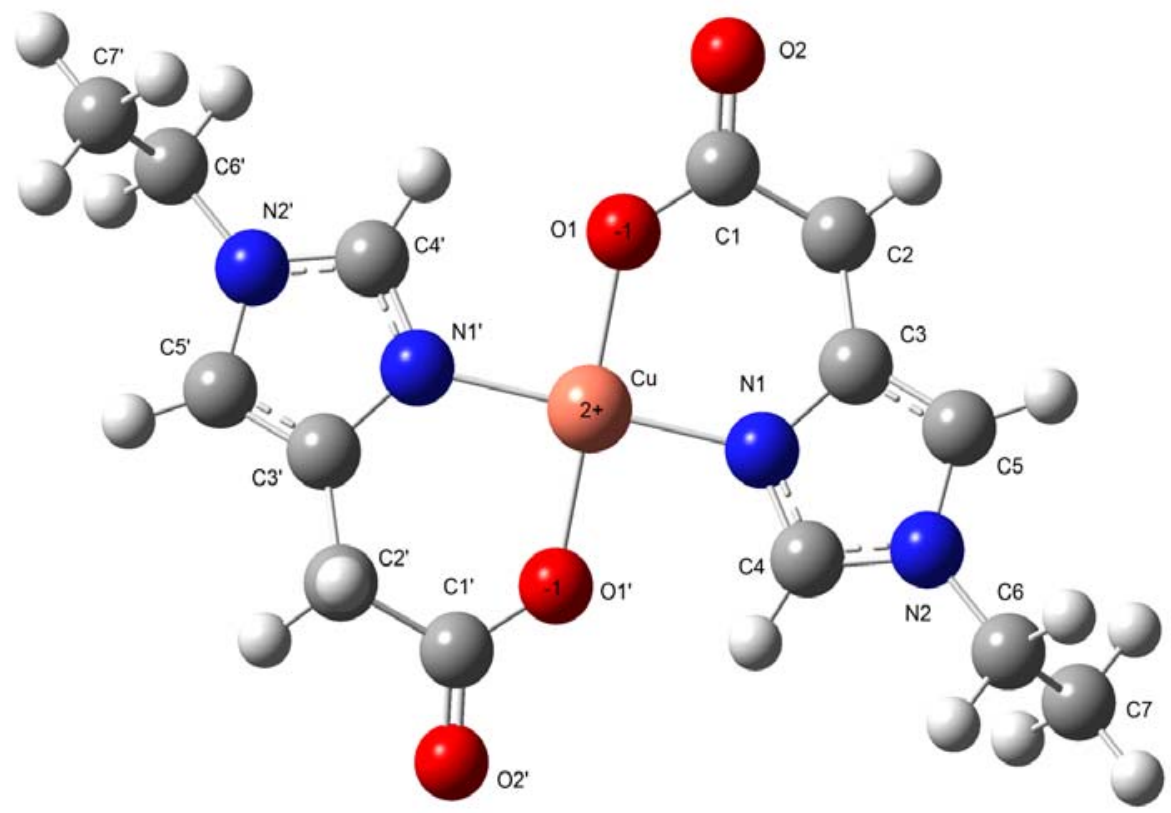

Figure 4.2: Molecular structure of the $\left[\mathrm{Cu}(\mathrm{IAC})_{2}\right]$ complex. The following colors salmon, gray, red, and white, represent $\mathrm{Cu}, \mathrm{C}, \mathrm{O}, \mathrm{N}$, and $\mathrm{H}$ atoms, respectively. 
Table 4.1: DFT cal culated structural parameters of the $\left[\mathrm{Cu}(\mathrm{IA})_{2}\right]$ complex and maximum absolute errors $^{\mathrm{a}}$ (MAE) for bond lengths (in $\AA$ ) and bond angles (in ${ }^{\circ}$ ). Cal culations are done with LanL2DZ LL2DZ) employed on Cu and 6-31G* basis set on ligand atoms.

\begin{tabular}{lcccccc}
\hline \hline $\begin{array}{l}\text { LL2DZ/6-31G* }^{*} \\
\text { Atoms }\end{array}$ & $\begin{array}{c}\text { BP86 } \\
\text { Bond lengths }\end{array}$ & MAE & Bond lengths & MAE & Bond lengths & MAE \\
\hline Cu-N1 & 1.981 & 0.03 & 1.992 & 0.04 & 1.977 & 0.03 \\
Cu-O1 & 1.981 & 0.00 & 1.939 & 0.04 & 1.951 & 0.03 \\
O1-C5 & 1.312 & 0.04 & 1.306 & 0.03 & 1.299 & 0.02 \\
O2-C5 & 1.237 & 0.01 & 1.223 & 0.03 & 1.224 & 0.02 \\
N1-C1 & 1.335 & 0.01 & 1.324 & 0.00 & 1.322 & 0.01 \\
N1-C3 & 1.391 & 0.00 & 1.384 & 0.00 & 1.379 & 0.01 \\
N2-C1 & 1.360 & 0.13 & 1.350 & 0.12 & 1.347 & 0.12 \\
N2-C2 & 1.391 & 0.01 & 1.386 & 0.01 & 1.381 & 0.00 \\
C2-C3 & 1.384 & 0.02 & 1.372 & 0.00 & 1.371 & 0.00 \\
C3-C4 & 1.498 & 0.01 & 1.496 & 0.01 & 1.485 & 0.01 \\
C4-C5 & 1.555 & 0.03 & 1.549 & 0.03 & 1.539 & 0.02 \\
\hline Atoms & Bond angles & MAE & Bond angles & MAE & Bond angels & MAE \\
\hline N1-Cu-O1 & 91.6 & 0.5 & 91.6 & 0.5 & 91.6 & 0.5 \\
N1'-Cu-O1 & 88.4 & 0.5 & 88.4 & 0.5 & 88.4 & 0.5 \\
C3-N1-Cu & 126.3 & 3.8 & 126.0 & 3.5 & 126.0 & 3.5 \\
O1-C5-O2 & 123.1 & 0.0 & 123.9 & 0.8 & 123.5 & 0.4 \\
O2-C5-C4 & 118.6 & 0.6 & 118.3 & 0.8 & 118.3 & 0.8 \\
C2-N1-C3 & 108.2 & 1.4 & 108.2 & 1.4 & 108.1 & 1.3 \\
C2-C3-N1 & 107.9 & 0.6 & 107.9 & 0.6 & 108.0 & 0.5 \\
C3-C2-N2 & 106.1 & 0.1 & 106.1 & 0.1 & 106.1 & 0.1 \\
C1-N2-C2 & 108.5 & 0.2 & 108.4 & 0.0 & 108.4 & 0.1 \\
N1-C1-N2 & 109.3 & 1.0 & 109.4 & 0.9 & 109.4 & 0.9 \\
\hline \hline
\end{tabular}

a MAE val ues are cal culated using X-ray data from Ref. 123

${ }^{b}$ Parameters related by inversion center are omitted.

All cal culated bond distances (for which maximum absol ute error does not exceed $0.15 \AA$ ) and angles (with maximum absolute error of $4^{\circ}$ ) agrees very well with corresponding values from X-ray data. ${ }^{123}$ Although optimized structural parameters of $\left[\mathrm{Cu}(\mathrm{IA})_{2}\right]$ are clearly related by the inversion center, within the default setup for the geometry optimization Gaussian does not identify $\mathrm{C}_{\mathbf{i}}$. Instead, the lowest point group symmetry is assigned, i.e., $\mathrm{C}_{1}$, 
and all the calculations are performed without taking into account any symmetry. In the case of calculations with BP86 functional a larger basis set for $\mathrm{Cu}$ actually leads to less accurate results.

Table 4.2: DFT calculated structural parameters of the $\left[\mathrm{Cu}(\mathrm{IA})_{2}\right]$ complex and maximum absolute errors ${ }^{\mathrm{a}}$ (MAE) for bond lengths (in $\AA$ ) and bond angles (in ${ }^{\circ}$ ). Cal cul ations are done with Stuttgart RSC (SRSC) employed on Cu and 6-31G* basis set on ligand atoms.

\begin{tabular}{lcccccc}
\hline \hline $\begin{array}{l}\text { SRSC/6-31G* } \\
\text { Atoms }\end{array}$ & \multicolumn{2}{c}{ BP86 } & \multicolumn{5}{c}{ B3LYP } & \multicolumn{4}{c}{ M06L } \\
Bond lengths & MAE & Bond lengths & MAE & Bond lengths & MAE \\
\hline Cu-N1 & 1.973 & 0.02 & 1.989 & 0.04 & 1.974 & 0.02 \\
Cu-O1 & 1.969 & 0.01 & 1.933 & 0.05 & 1.945 & 0.03 \\
O1-C5 & 1.316 & 0.04 & 1.308 & 0.03 & 1.302 & 0.03 \\
O2-C5 & 1.235 & 0.01 & 1.222 & 0.03 & 1.223 & 0.03 \\
N1-C1 & 1.335 & 0.01 & 1.324 & 0.00 & 1.323 & 0.01 \\
N1-C3 & 1.391 & 0.00 & 1.384 & 0.00 & 1.379 & 0.01 \\
N2-C1 & 1.359 & 0.13 & 1.350 & 0.12 & 1.347 & 0.12 \\
N2-C2 & 1.391 & 0.01 & 1.386 & 0.01 & 1.381 & 0.00 \\
C2-C3 & 1.383 & 0.02 & 1.372 & 0.00 & 1.370 & 0.00 \\
C3-C4 & 1.497 & 0.01 & 1.496 & 0.01 & 1.485 & 0.01 \\
C4-C5 & 1.555 & 0.03 & 1.548 & 0.03 & 1.539 & 0.02 \\
\hline Atoms & Bond angles & MAE & Bond angles & MAE & Bond angels & MAE \\
\hline N1-Cu-O1 & 92.0 & 0.9 & 91.9 & 0.7 & 91.9 & 0.8 \\
N1'-Cu-O1 & 88.0 & 0.9 & 88.1 & 0.8 & 88.1 & 0.8 \\
C3-N1-Cu & 126.0 & 3.5 & 125.7 & 3.2 & 125.8 & 3.3 \\
O1-C5-O2 & 123.2 & 0.0 & 123.8 & 0.7 & 123.6 & 0.5 \\
O2-C5-C4 & 118.7 & 0.5 & 118.4 & 0.7 & 118.4 & 0.8 \\
C2-N1-C3 & 108.1 & 1.3 & 108.1 & 1.3 & 108.0 & 1.2 \\
C2-C3-N1 & 108.0 & 0.6 & 108.0 & 0.6 & 108.0 & 0.5 \\
C3-C2-N2 & 106.1 & 0.1 & 106.1 & 0.1 & 106.1 & 0.1 \\
C1-N2-C2 & 108.5 & 0.2 & 108.4 & 0.0 & 108.4 & 0.1 \\
N1-C1-N2 & 109.3 & 1.0 & 109.5 & 0.8 & 109.4 & 0.9 \\
\hline \hline
\end{tabular}

${ }^{\text {a }}$ MAE values are cal culated using X-ray data from Ref. 123

${ }^{b}$ Parameters related by inversion center are omitted. 
This trend is particularly evident for bond angles. For instance, the N1-Cu-O1 angle increases from $91.6^{\circ}$ to $92.0^{\circ}$ for cal culations with [6s5p3d] basis set (involving an extra $3 \mathrm{~s}$, $2 p$ and $1 d$ basis functions) and to $92.2^{\circ}$ within the largest [5s4p3d1f] basis set. As this angle widens, the first coordination shell of $\mathrm{Cu}(\mathrm{II})$ differs more from the ideal square planar symmetry and distorting the structure of the complex.

Table 4.3: DFT cal culated structural parameters of the $\left[\mathrm{Cu}(\mathrm{IA})_{2}\right]$ complex and maximum absolute errors $^{\mathrm{a}}$ (MAE) for bond lengths (in $\AA$ ) and bond angles (in ${ }^{\circ}$ ). Cal culations are done with cc-pVDZ-PP (CCDPP) employed on $\mathrm{Cu}$ and 6-31G* basis set on ligand atoms.

\begin{tabular}{|c|c|c|c|c|c|c|}
\hline CCDPP/6-31G* & BP86 & & B3LYP & & M06L & \\
\hline Atoms $^{b}$ & Bond lengths & MAE & Bond lengths & MAE & Bond lengths & MAE \\
\hline Cu-N1 & 1.965 & 0.01 & 1.924 & 0.03 & 1.972 & 0.02 \\
\hline $\mathrm{Cu}-\mathrm{O} 1$ & 1.954 & 0.02 & 1.983 & 0.00 & 1.938 & 0.04 \\
\hline O1-C5 & 1.315 & 0.04 & 1.306 & 0.03 & 1.301 & 0.02 \\
\hline O2-C5 & 1.235 & 0.01 & 1.222 & 0.03 & 1.223 & 0.03 \\
\hline $\mathrm{N} 1-\mathrm{C} 1$ & 1.336 & 0.01 & 1.325 & 0.00 & 1.323 & 0.01 \\
\hline N1-C3 & 1.391 & 0.00 & 1.384 & 0.00 & 1.379 & 0.01 \\
\hline N2-C1 & 1.359 & 0.13 & 1.350 & 0.12 & 1.347 & 0.12 \\
\hline N2-C2 & 1.391 & 0.01 & 1.386 & 0.01 & 1.381 & 0.00 \\
\hline C2-C3 & 1.383 & 0.02 & 1.372 & 0.00 & 1.370 & 0.00 \\
\hline C3-C4 & 1.497 & 0.01 & 1.496 & 0.01 & 1.485 & 0.01 \\
\hline $\mathrm{C} 4-\mathrm{C} 5$ & 1.556 & 0.04 & 1.549 & 0.03 & 1.540 & 0.02 \\
\hline Atoms $^{b}$ & Bond angles & MAE & Bond angles & MAE & Bond angels & MAE \\
\hline N1-Cu-O1 & 92.2 & 1.1 & 92.0 & 0.8 & 92.0 & 0.9 \\
\hline N1'-Cu-O1 & 87.8 & 1.1 & 88.1 & 0.8 & 88.0 & 0.9 \\
\hline C3-N1-Cu & 126.1 & 3.6 & 125.7 & 3.2 & 125.7 & 3.2 \\
\hline $\mathrm{O} 1-\mathrm{C} 5-\mathrm{O} 2$ & 123.3 & 0.2 & 123.9 & 0.8 & 123.7 & 0.6 \\
\hline O2-C5-C4 & 118.5 & 0.6 & 118.2 & 0.9 & 118.2 & 0.9 \\
\hline $\mathrm{C} 2-\mathrm{N} 1-\mathrm{C} 3$ & 108.1 & 1.3 & 108.1 & 1.3 & 108.0 & 1.2 \\
\hline $\mathrm{C} 2-\mathrm{C} 3-\mathrm{N} 1$ & 108.0 & 0.6 & 108.0 & 0.6 & 108.0 & 0.5 \\
\hline C3-C2-N2 & 106.1 & 0.1 & 106.1 & 0.1 & 106.1 & 0.1 \\
\hline C1-N2-C2 & 108.6 & 0.2 & 108.4 & 0.0 & 108.4 & 0.1 \\
\hline N1-C1-N2 & 109.3 & 1.0 & 109.5 & 0.8 & 109.4 & 0.9 \\
\hline
\end{tabular}

a MAE val ues are cal culated using X-ray data from Ref. 123

${ }^{b}$ Parameters related by inversion center are omitted. 
In general the bigger the basis set the shorter the $\mathrm{Cu}-\mathrm{O} 1$ distance is. This trend can be observed for the Cu-N1 distance, even though this bond is less affected; this implies that $\mathrm{Cu}$ $\mathrm{N} 1$ bond is more shorten then $\mathrm{Cu}-\mathrm{O} 1$ is. The $\mathrm{Cu}-\mathrm{O} 1$ distance is al so predicted to be shorter using both LL2DZ and SRSC via B3LYP and M06L cal cul ations. This result is in disagreement with the experimental data where $\mathrm{Cu}-\mathrm{N} 1$ is 1.950(1) $\AA$ and $\mathrm{Cu}-\mathrm{O} 1$ is 1.978(1) $\AA .{ }^{123}$ In fact, only B3LYP results using CCDPP predicted properly these distances as well as providing the most accurate results in terms of MAEs.

For this reason the B3LYP functional in combination with cc-pVDZ-PP ECP and the [5s4p3d1f] basis set for Cu was chosen to reevaluate the cartesian coordi nates while changing basis sets for the ligand atoms. Results from this set of cal culations are shown in $\mathrm{Ta}$ ble 4.4 and Table 4.5. It turns out that increasing the size of basis sets on $\mathrm{H}, \mathrm{C}, \mathrm{N}$ and $\mathrm{O}$ atoms does not necessarily improve the accuracy of the structural parameters; for example, $\mathrm{Cu}-\mathrm{O} 1$ is shorter than $\mathrm{Cu}-\mathrm{N} 1$ bond length for all basis sets larger than $6-31 \mathrm{G}^{*}$. Bond angles are slightly better when this basis set is augmented, i.e., with diffuse functions $\left(6-31+G^{*}\right)$, but the Cu-O1 distance is shorter (1.935 ̊) than Cu-N1 (1.981 ̊̊).

Additional polarized basis functions worsen the accuracy of computed bond lengths and angles. For instance, the error for the $\mathrm{Cu}-\mathrm{O} 1$ distance obtained within $6-31 \mathrm{G}^{*}$ basis set for ligand atoms (see Tables 4.4) increased by $0.05 \AA$ at $6-31 G^{* *}$ level while going from $6-31+G^{*}$ to $6-31+G^{* *}$ sets off the O2-C5-C4 angle by $2.2^{\circ}$. The $6-31++G^{* *}$ basis set does not change bond distances and angles obtained with $6-31+G^{* *}$, except of the O2$\mathrm{C} 5-\mathrm{C} 4$ angle. This structural parameter increases from $116^{\circ}$ at $6-31+\mathrm{G}^{* *}$ level to $118^{\circ}$ at $6-31++G^{* *}$ level (note that values $118^{\circ}$ were also found with other tested herein basis sets employed on ligand atoms). Based on these observations one can conclude that no advantage is noticed in increasing the size of the basis set for ligand atoms beyond 6-31G* . Nevertheless, using 6-31+G* can be useful for achieving better accuracy for the absorption spectra. 
Table 4.4: Structural parameters of the $\left[\mathrm{Cu}(\mathrm{IA})_{2}\right]$ complex obtained with B3LYP/CCDPP with respect to different basis sets for ligand atoms. Maximum absolute errors ${ }^{a}$ (MAE) in Åis al so reported.

\begin{tabular}{|c|c|c|c|c|c|c|c|c|}
\hline \multirow[b]{2}{*}{ Atoms $^{b}$} & \multicolumn{2}{|l|}{$6-31 G^{* *}$} & \multicolumn{2}{|l|}{$6-31+G^{*}$} & \multicolumn{2}{|l|}{$6-31+G^{* *}$} & \multicolumn{2}{|l|}{$6-31++G^{* *}$} \\
\hline & Value $(\AA)$ & MAE & Value $(\AA)$ & MAE & Value in $(\AA)$ & MAE & Value $(\AA)$ & MAE \\
\hline Cu-N1 & 1.982 & 0.03 & 1.982 & 0.03 & 1.981 & 0.03 & 1.981 & 0.03 \\
\hline Cu-01 & 1.925 & 0.05 & 1.935 & 0.04 & 1.936 & 0.04 & 1.936 & 0.04 \\
\hline O1-C5 & 1.306 & 0.03 & 1.305 & 0.03 & 1.305 & 0.03 & 1.305 & 0.03 \\
\hline O2-C5 & 1.222 & 0.03 & 1.227 & 0.02 & 1.227 & 0.02 & 1.227 & 0.02 \\
\hline N1-C1 & 1.325 & 0.00 & 1.326 & 0.00 & 1.326 & 0.00 & 1.326 & 0.00 \\
\hline N1-C3 & 1.384 & 0.00 & 1.385 & 0.00 & 1.385 & 0.00 & 1.385 & 0.00 \\
\hline N2-C1 & 1.349 & 0.12 & 1.351 & 0.12 & 1.351 & 0.12 & 1.351 & 0.12 \\
\hline N2-C2 & 1.386 & 0.01 & 1.387 & 0.01 & 1.387 & 0.01 & 1.387 & 0.01 \\
\hline C2-C3 & 1.372 & 0.00 & 1.373 & 0.01 & 1.373 & 0.01 & 1.373 & 0.01 \\
\hline C3-C4 & 1.496 & 0.01 & 1.497 & 0.01 & 1.497 & 0.01 & 1.497 & 0.01 \\
\hline C4-C5 & 1.549 & 0.03 & 1.547 & 0.03 & 1.546 & 0.03 & 1.546 & 0.03 \\
\hline Atoms $^{b}$ & Value $\left({ }^{\circ}\right)$ & MAE & Value $\left(^{\circ}\right)$ & MAE & Value $\left({ }^{\circ}\right)$ & MAE & Value $\left({ }^{\circ}\right)$ & MAE \\
\hline N1-Cu- & 02.0 & 0.8 & 91.8 & 0.7 & 91.8 & 0.7 & 91.8 & 0.7 \\
\hline N1'-Cu- & O1 88.0 & 0.9 & 88.2 & 0.7 & 88.2 & 0.7 & 88.2 & 0.7 \\
\hline C3-N1-C & Ju 125.8 & 3.3 & 125.7 & 3.1 & 125.7 & 3.2 & 125.7 & 3.2 \\
\hline $\mathrm{O} 1-\mathrm{C} 5-\mathrm{C}$ & ว2 123.9 & 0.8 & 123.8 & 0.6 & 123.7 & 0.6 & 123.7 & 0.6 \\
\hline $\mathrm{O} 2-\mathrm{C} 5-\mathrm{C}$ & L4 118.1 & 1.0 & 118.4 & 0.8 & 116.1 & 3.0 & 118.3 & 0.8 \\
\hline C2-N1-C & 3 108.1 & 1.3 & 108.0 & 1.2 & 108.1 & 1.2 & 108.1 & 1.2 \\
\hline C2-C3-N & J1 108.0 & 0.6 & 108.0 & 0.5 & 108.0 & 0.5 & 108.0 & 0.5 \\
\hline C3-C2-N & V2 106.1 & 0.1 & 106.1 & 0.1 & 106.1 & 0.1 & 106.1 & 0.1 \\
\hline C1-N2-C & 108.4 & 0.1 & 108.4 & 0.1 & 108.4 & 0.1 & 108.4 & 0.1 \\
\hline N1-C1-N & V2 109.5 & 0.9 & 109.5 & 0.8 & 109.4 & 0.9 & 109.4 & 0.9 \\
\hline
\end{tabular}

${ }^{\text {a }}$ MAE values are cal culated using X-ray data from ref. ${ }^{123}$

${ }^{b}$ Parameters related by inversion center are omitted

The importance in the choice the basis set for ground-state properties is discussed later (Chapter 5). The augmentation was necessary due to the negative charge of the lithium monoxide ion. In fact, the high energy molecular orbitals of anions are likely to be more diffuse. This tendency is also observed for highly excited-states; ${ }^{144}$ therefore in order to find compromise between accuracy of predicted geometry (a ground-state property) and excitation energies, the $6-31+G^{*}$ basis set will be utilized for ligand atoms. 
The $\left[\mathrm{Cu}(\mathrm{IAC})_{2}\right]$ complex was optimized in vacuum, methanol $\left(\mathrm{CH}_{3} \mathrm{OH}\right)$ and dichloromethane $\left(\mathrm{CH}_{2} \mathrm{Cl}_{2}\right)$ using the B3LYP functional with CCDPP for $\mathrm{Cu}$ and $6-31+\mathrm{G}^{*}$ for ligand atoms; we will indicate this approach as B3LYP/CCDPP/6-31+G* . Results from this set of cal culations and comparison with the $X$-ray data ${ }^{120}$ are presented in Table 4.5. Experimental cartesian coordi nates were used as the starting point geometry. The accuracy of structural parameters optimized in vacuum is excellent considering that the actual crystal structure of the complex is $\mathrm{Cu}(\mathrm{IAC})_{2} \cdot 2 \mathrm{CH}_{3} \mathrm{OH}$. In the crystal two methanol molecules are weakly bonded with the metal center in the axial position, but such bonding is not included in the theoretical cal culations performed herein. Yet, the Maximum Absolute Errors (MAEs) do not exceed $0.04 \AA ̊$ for bond lengths and $4^{\circ}$ for bond angles; this seems to suggest that axial interactions are not important to properly describe the structure of this complex. Nevertheless, similarly to what observed for the $\mathrm{Cu}(\mathrm{II})$ complex with 4-imi dazole acetate, the $\mathrm{Cu}-\mathrm{O} 1$ distance is shorter than that of $\mathrm{Cu}-\mathrm{N} 1$. This is in disagreement with X-ray measurements, where the cooper-oxygen bond length is $1.976 \AA$ and the copper-nitrogen is $1.951 \AA .{ }^{120}$ The IEF-PCM used here to mimic methanol and dichloromethane is limited, because it takes into account only the dielectric constant and the refractive index of the solvent, nonethel ess a noticeable improvement in the atomic arrangement of $\left[\mathrm{Cu}(\mathrm{IAC})_{2}\right]$ complex (cal culated with B3LYP/CCDPP/6-31+G*) is observed in comparison with the results obtained in vacuum. This aspect can be treated as an additional confirmation that taking into account axial interactions is not essential. When in methanol the $\mathrm{Cu}-\mathrm{O} 1$ bond elongates by about $0.04 \AA$ and becomes slightly longer than the Cu-N1 bond, showing a good match with experimental value. Such result eliminates al most completely the MAE (i.e., $<0.001 \AA ̊$ ). It is interesting to note that these distances change the most during optimization in $\mathrm{CH}_{3} \mathrm{OH}$ and $\mathrm{CH}_{2} \mathrm{Cl}_{2}$. When in methanol, calculated accuracy for most of the bond angles also improves, e.g., C1-O1-Cu angle is $133.1^{\circ}$ obtained with an $\mathrm{MAE}=3.3^{\circ}$, which reduces to less than $1^{\circ}$. As an exception, the O2-C1-C2 angle calculated in vacuum $\left(118.3 \pm 1.5^{\circ}\right)$ was in better agreement with experiment than in methanol $\left(119.2 \pm 2.4^{\circ}\right)$. 
Table 4.5: Structural parameters of the $\left[\mathrm{Cu}(\mathrm{IAC})_{2}\right]$ complex cal culated in vacuum, $\mathrm{CH}_{3} \mathrm{OH}$, and $\mathrm{CH}_{2} \mathrm{Cl}_{2}$. Maximum absolute errors(MAE) for bond lengths (in $\AA$ ) and bond angles (in ${ }^{\circ}$ ) are also reported.

\begin{tabular}{lccccccc}
\hline \hline & $\begin{array}{c}\text { Experiment } \\
\text { Bond }\end{array}$ & $\begin{array}{c}\text { Vacuum } \\
\text { Bond }\end{array}$ & \multicolumn{3}{c}{$\begin{array}{c}\mathrm{CH}_{3} \mathrm{OH} \\
\text { Bond }\end{array}$} & \multicolumn{3}{c}{$\mathrm{CH}_{2} \mathrm{Cl}_{2}$} \\
Atoms & Bond & \\
lengths & lengths & MAE & lengths & MAE & lengths & MAE \\
\hline Cu-N1 & 1.951 & 1.978 & 0.03 & 1.972 & 0.02 & 1.975 & 0.02 \\
Cu-O1 & 1.976 & 1.939 & 0.04 & 1.976 & 0.00 & 1.972 & 0.00 \\
O1-C1 & 1.270 & 1.304 & 0.03 & 1.294 & 0.02 & 1.296 & 0.03 \\
O2-C1 & 1.248 & 1.228 & 0.02 & 1.244 & 0.00 & 1.241 & 0.01 \\
N1-C3 & 1.379 & 1.382 & 0.00 & 1.384 & 0.00 & 1.384 & 0.00 \\
N1-C4 & 1.328 & 1.328 & 0.00 & 1.332 & 0.00 & 1.332 & 0.00 \\
N2-C4 & 1.344 & 1.350 & 0.01 & 1.348 & 0.00 & 1.349 & 0.00 \\
N2-C5 & 1.381 & 1.388 & 0.01 & 1.385 & 0.00 & 1.386 & 0.00 \\
N2-C6 & 1.466 & 1.466 & 0.00 & 1.471 & 0.01 & 1.470 & 0.00 \\
C1-C2 & 1.526 & 1.547 & 0.02 & 1.536 & 0.01 & 1.537 & 0.01 \\
C2-C3 & 1.493 & 1.497 & 0.00 & 1.497 & 0.00 & 1.496 & 0.00 \\
C3-C5 & 1.364 & 1.374 & 0.01 & 1.372 & 0.01 & 1.372 & 0.01 \\
C6-C7 & 1.515 & 1.530 & 0.02 & 1.530 & 0.02 & 1.529 & 0.01 \\
\hline & Bond & Bond & & Bond & & Bond & \\
Atoms & angles & angles & MAE & angles & MAE & angels & MAE \\
\hline N1-Cu-O1 & 90.4 & 91.7 & 1.3 & 90.7 & 0.3 & 91.0 & 0.6 \\
N1'-Cu-O1 & 89.6 & 88.3 & 1.3 & 89.3 & 0.3 & 89.0 & 0.6 \\
C1-O1-Cu & 129.8 & 133.1 & 3.3 & 130.3 & 0.5 & 130.6 & 0.8 \\
C3-N1-Cu & 124.4 & 125.8 & 1.4 & 124.0 & 0.4 & 124.3 & 0.1 \\
C4-N1-Cu & 128.1 & 125.8 & 2.3 & 128.4 & 0.3 & 128.0 & 0.1 \\
O2-C1-O1 & 123.3 & 123.8 & 0.5 & 122.9 & 0.4 & 123.1 & 0.2 \\
O2-C1-C2 & 116.8 & 118.3 & 1.5 & 119.2 & 2.4 & 119.2 & 2.4 \\
O1-C1-C2 & 119.9 & 117.8 & 2.1 & 117.9 & 2.0 & 117.7 & 2.2 \\
C1-C2-C3 & 115.3 & 117.1 & 1.8 & 114.2 & 1.1 & 114.2 & 1.1 \\
C5-C3-C2 & 129.1 & 129.6 & 0.5 & 129.9 & 0.8 & 129.9 & 0.8 \\
N1-C3-C2 & 122.2 & 122.5 & 0.3 & 121.8 & 0.4 & 121.8 & 0.4 \\
N2-C6-C7 & 112.4 & 112.8 & 0.4 & 112.5 & 0.1 & 112.6 & 0.2 \\
C3-C5-N2 & 106.4 & 106.8 & 0.4 & 106.6 & 0.2 & 106.6 & 0.2 \\
C4-N2-C5 & 107.7 & 107.4 & 0.3 & 107.3 & 0.4 & 107.6 & 0.1 \\
C5-N2-C6 & 126.9 & 126.5 & 0.4 & 126.3 & 0.6 & 126.3 & 0.6 \\
N1-C4-N2 & 110.4 & 110.4 & 0.0 & 110.4 & 0.0 & 110.4 & 0.0 \\
\hline \hline
\end{tabular}

a X-ray data from Ref. 120

${ }^{b}$ Parameters related by inversion center are omitted 
The N1-Cu-O1 angle, which together with Cu-O1 and Cu-N1 bonds can be used to determine first coordination shell of $\mathrm{Cu}(\mathrm{II})$ as square planar symmetry, is closer to $90^{\circ}$ in methanol than in vacuum. For calculations with dichloromethane the $\mathrm{Cu}-\mathrm{O} 1$ distance is slightly shorter than in methanol but longer than in vacuum. This can be attributed to the smal ler dielectric constant of $\mathrm{CH}_{2} \mathrm{Cl}_{2}$. Namely, sice as $r \propto 1 / \varepsilon$, the bond length $(r)$ will ideally change by the factor of $\sim 1 / 33$ for methanol and $\sim 1 / 9$ for dichloromethane with respect to the value obtained in vacuum $(r \propto 1)$. The total effect on the bond length can be accounted for as $\Delta r=\frac{\left|r_{0}-r \varepsilon^{-1}\right|}{r_{0}} \cdot 100 \%$, where $r_{0}$ is the bond length computed in vacuum and $r$ is corresponding bond length obtained in a given solvent. Not all the bonds elongate when solvation effects are included; for example, the Cu-N1 distance is shorter in $\mathrm{CH}_{3} \mathrm{OH}$ both than in vacuum and in $\mathrm{CH}_{2} \mathrm{Cl}_{2}$. This result agrees with the assumption that overall solvation effects are smaller in dichloromethane than in methanol. Somewhat ambiguous results for some of the computed bond distances seems to occur with respect to different solvent; for instance, the N1-C4 bond is elongated by $0.004 \AA$ in both solvents. For bond angles no uniform trend can be found when solvents are involved. Some bond angles increase whereas others decrease in comparison to resul ts obtai ned in vacuum. Furthermore, the comparison between methanol and dichloromethane shows that few angles are not affected at all by the change from the less to more polar solvation environment.

\subsubsection{UV-Vis Spectra}

Geometries optimized at B3LYP/CCDPP/6-31+G* level of theory were used to calculate excitation energies and oscillator strengths via the standard xc functionals BP86, B3LYP and M06L. The most important electronic transitions selected from fifty computed excited states are shown in the Tables 4.6-4.10. Note that qual itative assignments of the computed transitions for $\left[\mathrm{Cu}(\mathrm{IA})_{2}\right]$ and $\left[\mathrm{Cu}(\mathrm{IAC})_{2}\right]$ complex is done based on the population analysis of molecular orbitals. 
Table 4.6: Selected electronic transitions and oscillator strengths (f) for the $\left[\mathrm{Cu}(\mathrm{IA})_{2}\right]$ complex from TDDFT calculations in vacuum. Assignment ${ }^{a}$ to the corresponding experimental absorption bands with determined errors ${ }^{b}(\Delta)$ for excitation energies (in eV) is al so reported. $\lambda$ is the wavel ength and EE stands for the excitation energy, cx-carboxylate, im-imidazole.

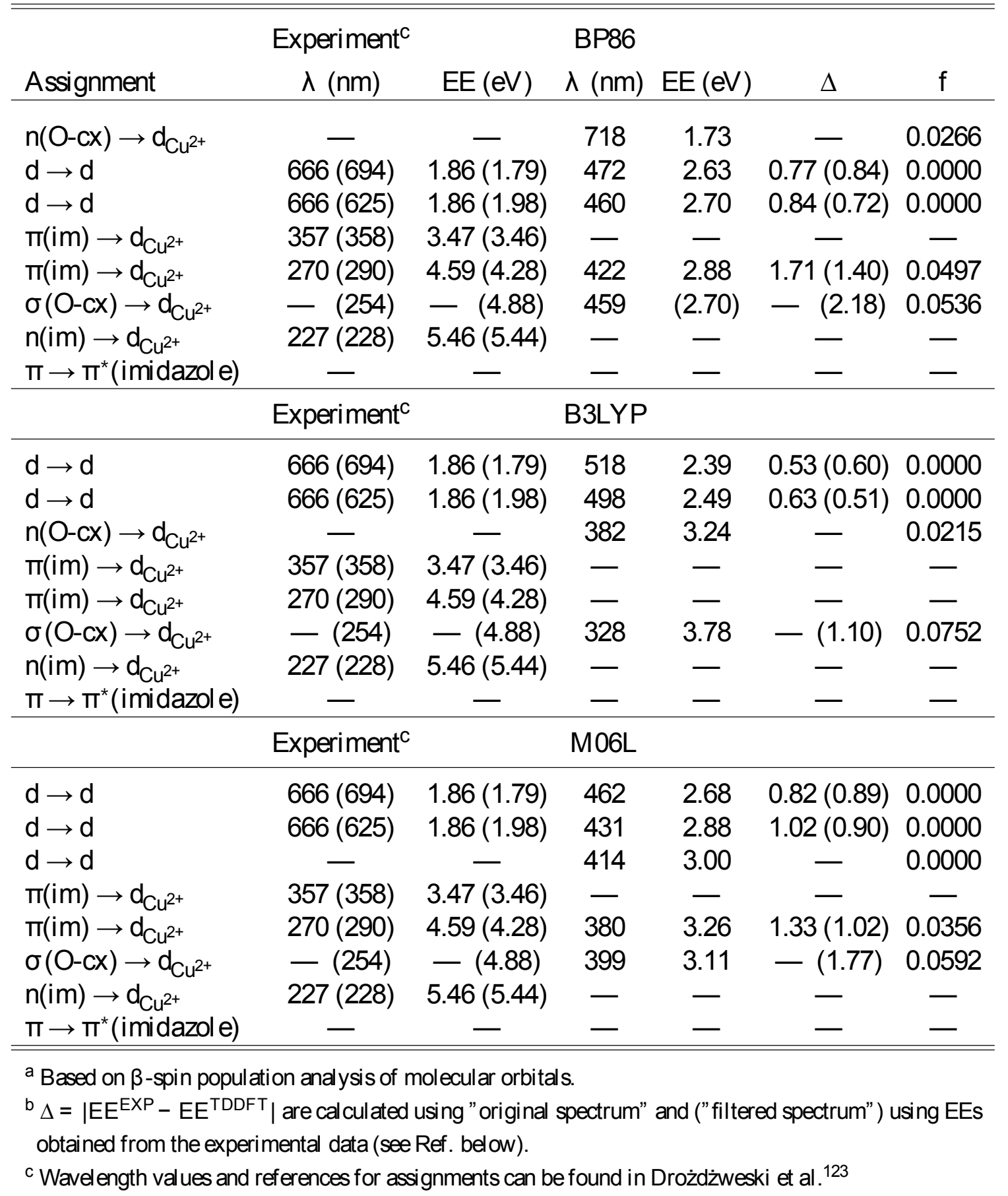


Hence in order to be able to define a given transition the following conditions should be met: (a) the Molecular Orbital (MO) pair defining a one-electron transition should contribute at least $50 \%$ to a given excited state; (b) the spin contamination cannot exceed $30 \%$ of the $S^{2}$ val ue, which for the pure doublet-to-doublet transitions is 0.75 .

As it can be seen in the Table 4.6 both quantitative and qualitative agreement between TDDFT results and experimental data is not favorable. In fact, the excitation energy of electronic transition assigned as $\mathrm{d}-\mathrm{d}$ is overestimated by about $0.5 \mathrm{eV}$ from cal culations performed with B3LYP and for the ligand-to-metal charge transfer (LMCT) transitions this functional performs even worst, i.e., only one of LMCT transitions from the reflectance spectrum ${ }^{123}$ was found with an absolute error of $1.1 \mathrm{eV}$. BP86 and M06L perform similarly despite the latter being a higher theory level: both the excitation energies of $d$ - $d$ transitions are overestimated by about $0.7-0.9 \mathrm{eV}$, while LMCT transitions are underestimated by about 1-2 eV. Given such large errors it is fair to admit that the standard xc functionals employed in this work failed to quantitatively describe both metal- and ligand-based transitions. For instance, the ligand-based $n$ (imidazole) $\rightarrow d_{C_{u^{2+}}}(L M C T)$ transition which in the experiment overlaps with the $\pi \rightarrow \pi^{*}$ (imidazole) is not found. One can argue that these results are not reliable since these transitions were computed at TDDFT level of theory within 50 excited states. Furthermore other issues might occur that influence the poor agrement; severe mixed character (i.e., different MO pairs can comparably contribute to a given transition) of the higher excited states, very low or no intensity at all ( $f<0.001$ or $f=0$ ), high spin contamination. All these aspects can make the identification not reliable. Yet some qual itative agreement can be found: for example, $d-d$ transitions are experimentally assigned to the absorption peak at about $2 \mathrm{eV}$ for the $\left[\mathrm{Cu}(\mathrm{IA})_{2}\right]$ complex. TDDFT cal cul ations al so predicts these transitions to be in this region. However, in experiment $d-d$ transitions have medium intensity, whereas for cal culations in the vacuum (gas phase) they are optically inactive. This is because LF transitions of complexes with a square planar geometry are Laporte forbidden, hence as expected we obtained zero oscillator strengths. 
In addition, as mentioned before, some excited states are missing and/or other spurious ones appear; such behavior of the standard $x c$ functional s in applications of adiabatic TDDFT is al so found in the study by Wu et al. ${ }^{132}$ Figure 4.3 shows the UV-Vis spectra of $\mathrm{Cu}(\mathrm{II})$ complex with 4-imidazole acetate computed with TDDFT.

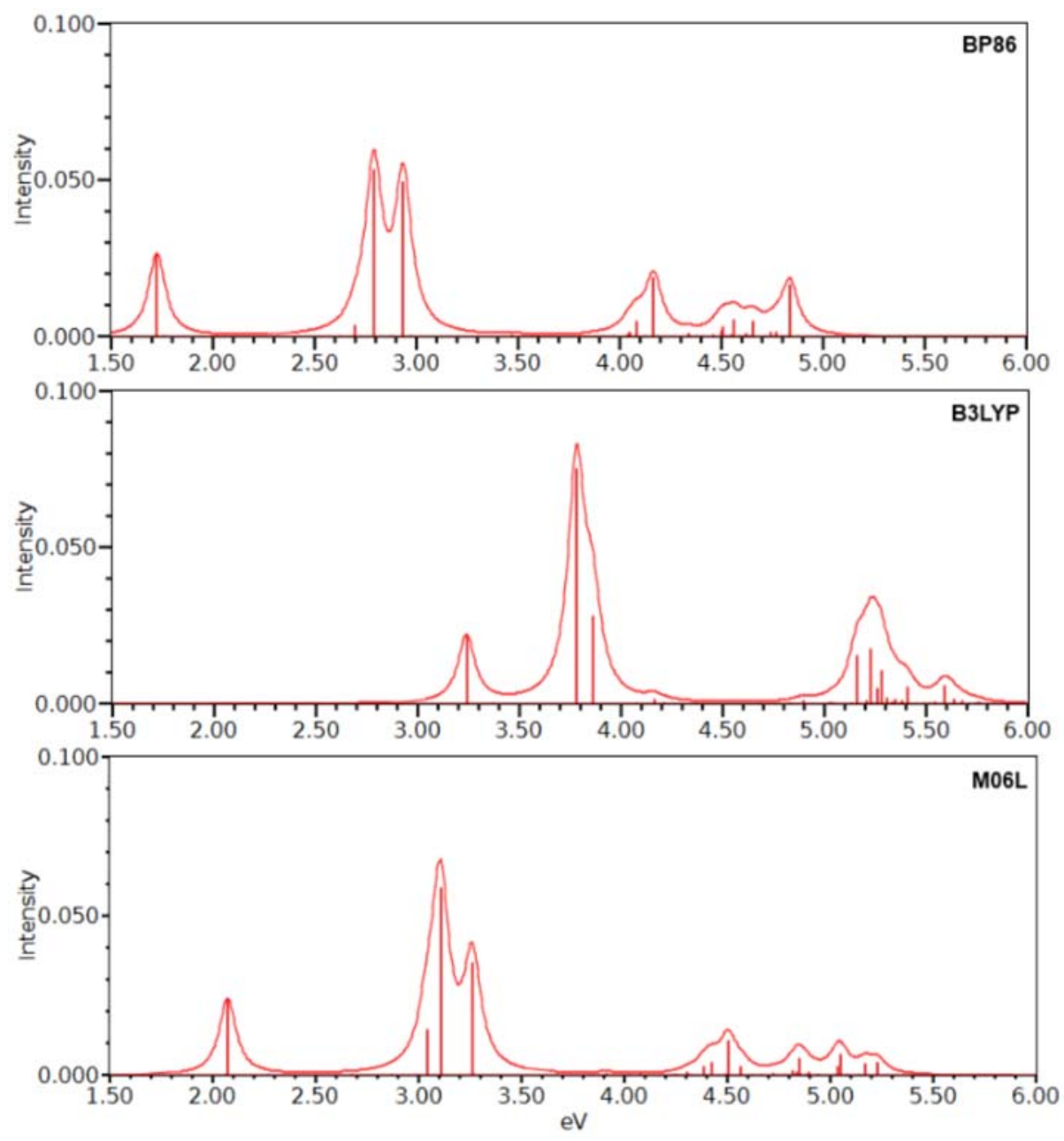

Figure 4.3: TDDFT UV-Vis spectra calculated for the $\left[\mathrm{Cu}(\mathrm{IA})_{2}\right]$ complex in the vacuum. Spectral line-shape is created by broadening lines with $0.05 \mathrm{eV}$ Half-Width Lorentzian. Intensity is given in terms of the oscillator strength, and is dimensionless.

It is evident noticed that the spectrum obtained with B3LYP differs significantly from those 
obtained with other functionals. The spectrum predicted with BP86 resembles the one calculated with M06L functional, although the same transitions found in the latter are red shifted with respect to BP86 values, e.g., the $\sigma$ (O-carboxylate) $\rightarrow \mathrm{d}_{\mathrm{Cu}^{2}}$ (LMCT) transition is computed at $444 \mathrm{~nm}$ and it shifts to $399 \mathrm{~nm}$ when M06L is employed. In general the worst results were obtained with the BP86 functional which in addition od giving a poor agreement with experiment produced spurious CT transition at $718 \mathrm{~nm}$ (n(O-carboxylate) $\rightarrow \mathrm{d}_{\mathrm{Cu}^{2+}}$ ). Such transition was determined by M06L and B3LYP at much shorter wavelength, $597 \mathrm{~nm}$ and $382 \mathrm{~nm}$, respectively. Furthermore, this transition was not found experimentally. We suspect that this peak may be covered by the broad and intense absorption observed in 240-450 nm the region. Moreover, for both the pure GGA functional s the thi rd lowest excited state, described by a dominating $\beta$-spin MO pair (HOMO- 2 and LUMO) cannot be properly assigned because HOMO- 2 has an even mixture of atomic orbitals of Cu-d and O-p. Interestingly enough, this transition is found as the first excited state with B3LYP functional and can be classified as mixed transition: $d-d$ (HOMO- $11 \rightarrow$ LUMO) and LMCT (HOMO- $2 \rightarrow$ LUMO).

The UV-Vis spectra of the Cu(II) complex with 1-ethyl-1H-imidazol-4-ylacetate were measured in solution; ${ }^{120}$ results from calculations in vacuum will be presented to verify the effectiveness of IEF-PCM used to mimic solvation effects of methanol and dichloromethane (Tables 4.7-4.10). Note that experimental UV-Vis spectrum of $\left[\mathrm{Cu}(\mathrm{IAC})_{2}\right]$ complex measured in methanol shows two distinct peaks with maximum absorption at $260 \mathrm{~nm}(4.77 \mathrm{eV})$ and $645 \mathrm{~nm}(1.92 \mathrm{eV})$. These two bands shift upon a change of solvent, i.e., for measurements in dichloromethane the first peak red shifts to $276 \mathrm{~nm}(4.49 \mathrm{eV})$ and the second blue shifts to $575 \mathrm{~nm}(2.16 \mathrm{eV})$. Such shifts were not reproduced by TDDFT calculation with solvation model employed in this study. The only $d$-d transitions for which predicted wavel ength changed are: HOMO- $7 \rightarrow$ LUMO (cal cul ated with BP86), and HOMO- $10 \rightarrow$ LUMO (calculated with B3LYP). However, the former red shifts by about $46 \mathrm{~nm}$, in disagreement with experimental observations. ${ }^{120}$ 


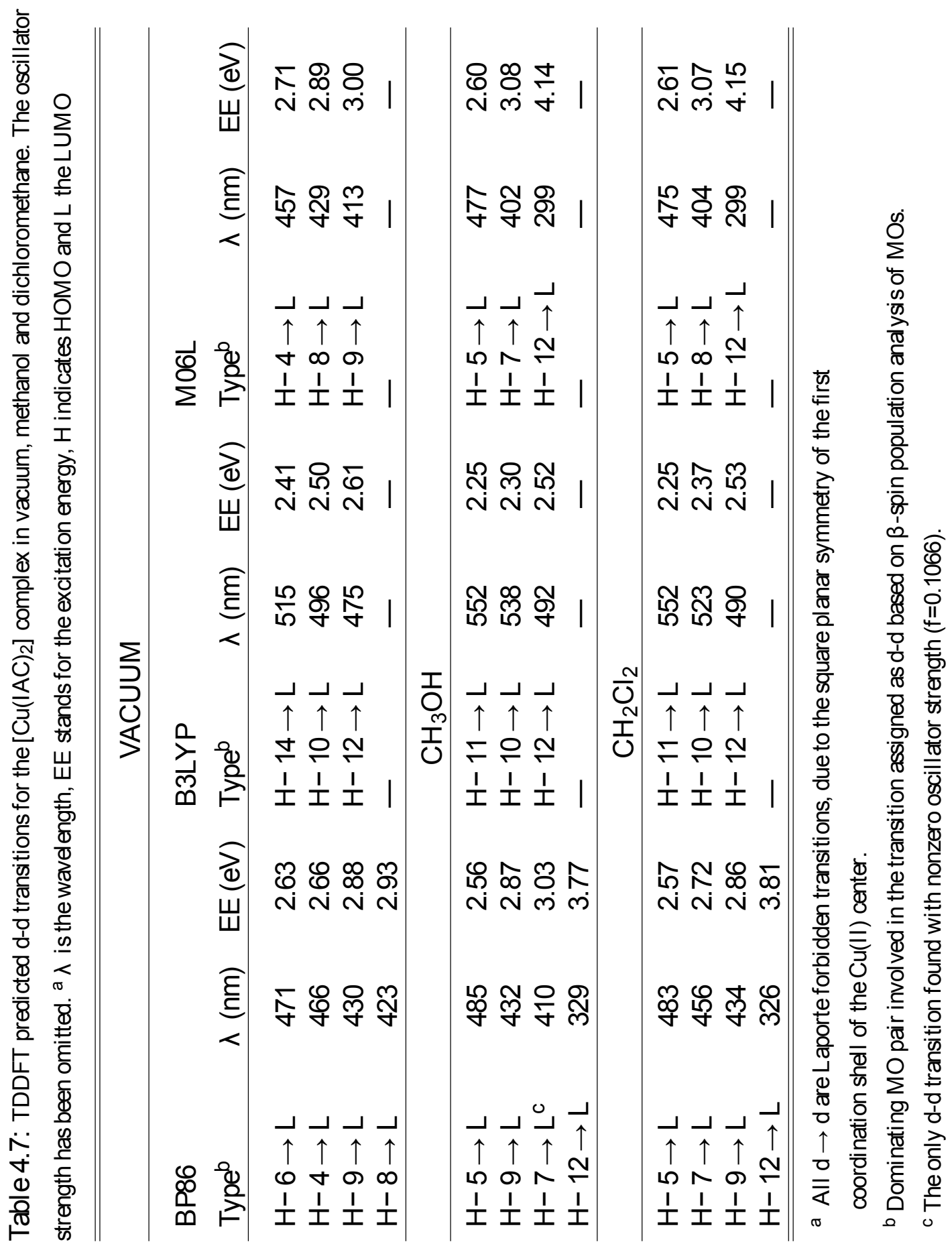


B3LYP correctly predicts the blue shift, despite being too small, i.e., only about $15 \mathrm{~nm}$ instead of $75 \mathrm{mn}$.

The shape of HOMO-10 is similar and it resembles the $d_{z^{2}}$ orbital of the $\mathrm{Cu}(\mathrm{II})$ center. This orbital will interact with methanol molecules in the solution. Yet, because IEF-PCM does not include any other information about the solvent apart its dielectric constant, it is not surprising that this model is insufficient to mimic all solvation effects. Nevertheless, in comparison with results obtained in vacuum a significant improvement is achieved in terms of accuracy of the predicted $d-d$ transitions for the $\left[\mathrm{Cu}(\mathrm{IAC})_{2}\right]$ complex. This is a clear indication of the fact that computed UV-VIS spectra of solvated metal-organic complexes should take into account solvation effects because even the si mplest model improves the results.

The most important LMCT transitions predicted with TDDFT is the $\sigma$ (O-carboxylate) $\rightarrow \mathrm{d}_{\mathrm{Cu}^{2+}}$, which according to solvent may be assigned to the experimental absorption band at $260 \mathrm{~nm}$ or $276 \mathrm{~nm}$. Fig. 4.4 shows the best UV-Vis spectra predicted with standard xc functionals in vacuum, methanol and dichloromethane. Tables (4.7-4.10) lists the most intense ligand-based transitions.

The accuracy for LMCT transitions is much worse than for $\mathrm{d}-\mathrm{d}$ transition. Moreover, for many of the highest excited states it is impossible to identify the electron donor and/or acceptor as the atomic coefficients describing the character of the dominating molecular orbital pair involved in a given transition; these turns out to be very similar. For example, $\sigma$ (O-carboxylate)/ $\pi\left(\right.$ imidazole) $\rightarrow \mathrm{d}_{\mathrm{Cu}^{2+}}$ transition predicted by B3LYP in vacuum at $325 \mathrm{~nm}$ (Table 4.9) involves donor with contributions from C-p (20-22\%) of imidazole and O-p (20-22\%) of carbonyl moiety of carboxylate. Similarly for calculations with BP86 in methanol where this LMCT transition was found at $505 \mathrm{~nm}$ (Table 4.8), and with M06L in dichloromethane at $439 \mathrm{~nm}$ (Table 4.10). 

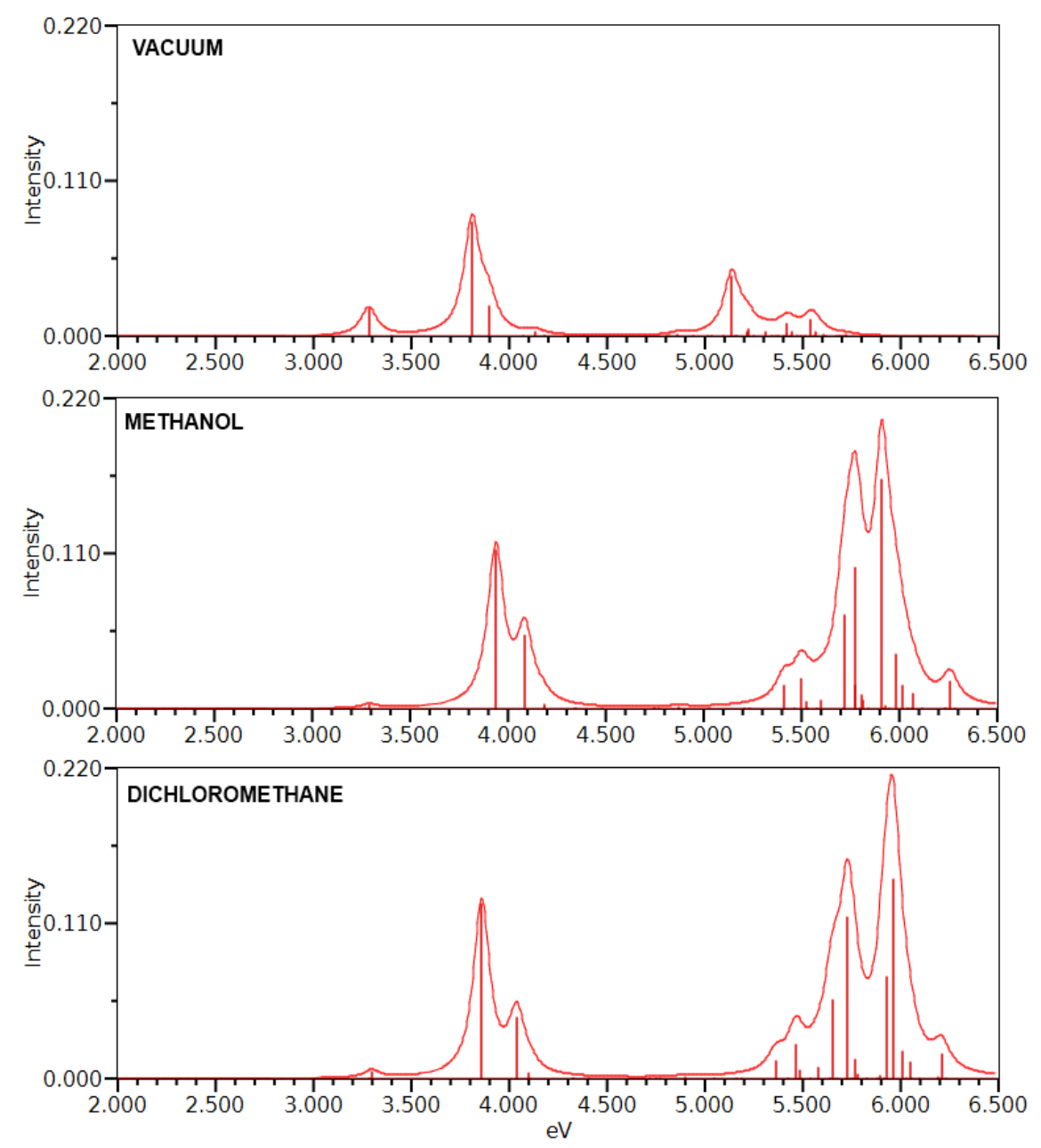

Figure 4.4: TD-B3LYP UV-Vis spectra calculated for the $\left[\mathrm{Cu}(\mathrm{IAC})_{2}\right]$ complex in vacuum, $\mathrm{CH}_{3} \mathrm{OH}$ and $\mathrm{CH}_{2} \mathrm{Cl}_{2}$. Spectral line-shape is created by broadening lines with $0.05 \mathrm{eV}$ Half-Width Lorentzian. Intensity is given in terms of the oscillator strength, and is dimensionless.

It is possible to find either LMCT transition (based on experimental assignments for the $\left[\mathrm{Cu}(\mathrm{IA})_{2}\right]$ complex $\left.{ }^{123}\right)$ in the spectrum range between $250-360 \mathrm{~nm}$ the theoretical absorption peak at $325 \mathrm{~nm}$ can be interpret as one of these two possi ble transitions. With such scenario the quantitative comparison with experimental data becomes unreliable. In addition to the problem of highly mixed character of the predicted by TDDFT electronic transitions in few cases, a significant spin contamination occurred. This conclusion is based on the 
analysis of the expectation value for $S^{2}$ (where $S=\overline{S(S+1)}$ ). For instance, for a very intense Cu-p/n(im) $\rightarrow \mathrm{d}_{\mathrm{Cu}^{2+}}$ transition employing M06L functional $\mathrm{S}^{2}=1.417$ in methanol, and 0.933 in dichloromethane. Unfortunately values of $S^{2}$ are not available for B3LYP results (the older version of the Gaussian program could not provide information on spin), but it can be expected that mixing HF exchange will cause even higher spin contamination. ${ }^{4}$

Table 4.8: BP86 predicted LMCT transitions with corresponding oscillator strength (f) for the $\left[\mathrm{Cu}(\mathrm{IAC})_{2}\right]$ complex in vacuum, methanol and dichloromethane. $\lambda$ is the wavel ength, EE is the excitation energy, cx stands for carboxylate, im for imidazole and et for ethyl group.

\begin{tabular}{lrcc}
\hline \hline \multicolumn{5}{c}{ VACUUM } \\
\hline Type $^{\mathrm{a}}$ & $\lambda(\mathrm{nm})$ & $\mathrm{EE}(\mathrm{eV})$ & $\mathrm{f} \times 10^{-2}$ \\
\hline $\mathrm{n}(\mathrm{O}-\mathrm{cx}) \rightarrow \mathrm{d}_{\mathrm{Cu}^{2+}}$ & 704 & 1.76 & 2.55 \\
$\mathrm{n} / \sigma(\mathrm{O}-\mathrm{cx}) \rightarrow \mathrm{d}_{\mathrm{Cu}^{2+}}$ & 448 & 2.77 & 2.65 \\
$\pi(\mathrm{im}) \rightarrow \mathrm{d}_{\mathrm{Cu}^{2+}}$ & 422 & 2.94 & 7.00 \\
\hline \multicolumn{5}{c}{} \\
\hline$\sigma(\mathrm{O}-\mathrm{cx}) / \pi(\mathrm{im}) \rightarrow \mathrm{d}_{\mathrm{Cu}^{2+}} \mathrm{CH}_{3} \mathrm{OH}$ \\
$\mathrm{n}(\mathrm{im}) \rightarrow \mathrm{d}_{\mathrm{Cu}^{2+}}$ & 505 & 2.46 & 2.43 \\
$\sigma(\mathrm{C}-\mathrm{et}) \rightarrow \mathrm{d}_{\mathrm{Cu}^{2+}}$ & 261 & 4.75 & 8.27 \\
& 261 & 5.06 & 2.54 \\
\hline $\mathrm{n}(\mathrm{O}-\mathrm{cx}) \rightarrow \mathrm{d}_{\mathrm{Cu}^{2+}}^{\mathrm{b}}$ & $\mathrm{CH}_{2} \mathrm{Cl}{ }_{2}$ & & \\
$\sigma(\mathrm{O}-\mathrm{cx}) \rightarrow \mathrm{d}_{\mathrm{Cu}^{2+}}$ & 676 & 1.84 & 1.26 \\
\hline \hline
\end{tabular}

a Based on $\beta$-spin population analysis of the dominating MO pair involved in a given transition.

${ }^{b}$ From a different occupied molecular orbital. 
Table 4.9: B3LYP predicted LMCT transitions with corresponding oscillator strength (f) for the $\left[\mathrm{Cu}(\mathrm{IAC})_{2}\right]$ complex in vacuum, methanol and dichloromethane. $\lambda$ is the wavel ength, EE is the excitation energy , cx stands for carboxylate, im for imidazole and et for ethyl group.

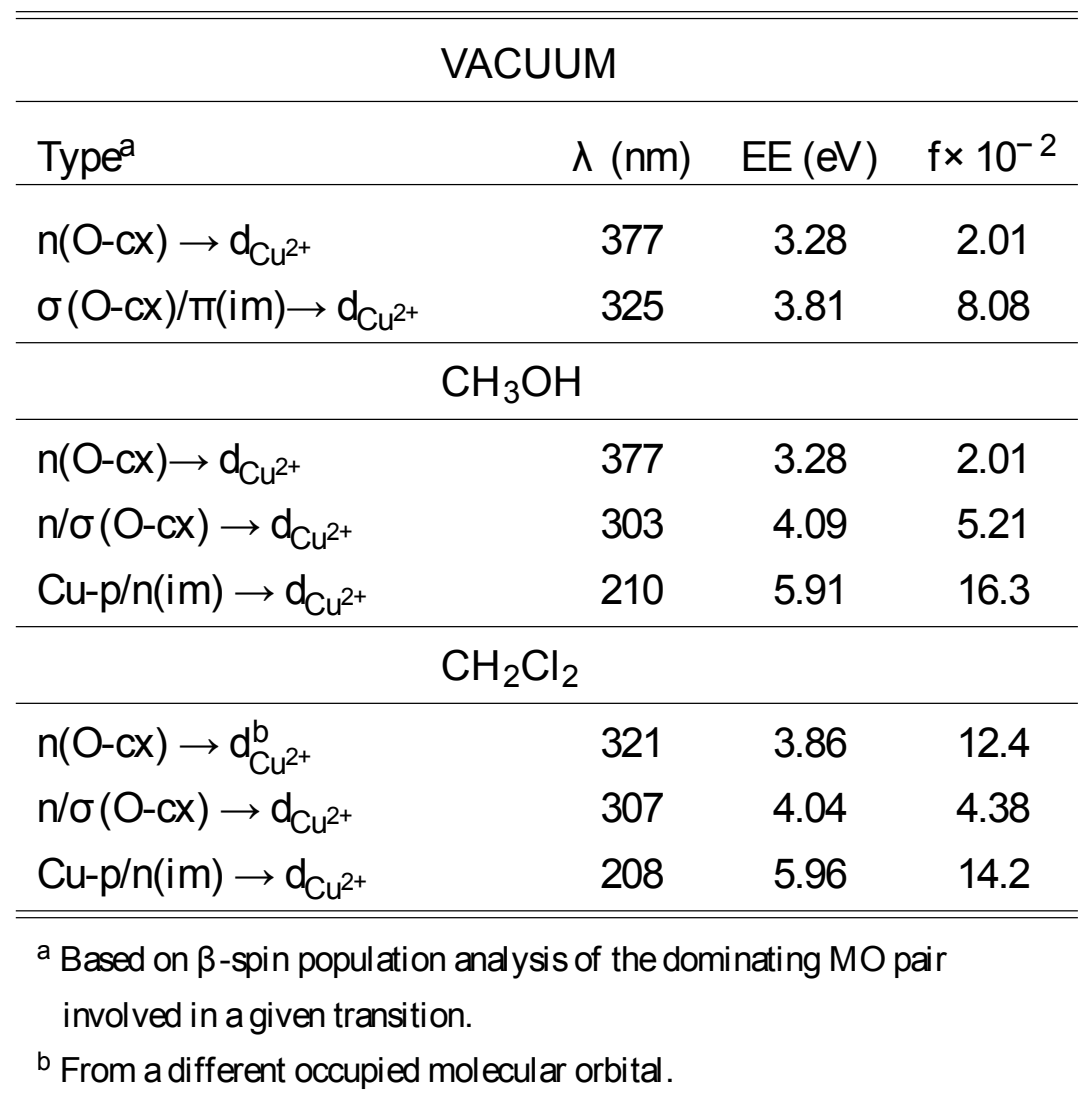

Furthermore, the only possible state with $S^{2} 2$ would be a triplet state but it is highly unlikely that such excited state could appear from a doublet ground state as it would require three or more electrons to be simultaneously excited. The most common type of excited states from a doublet ground state is the one dominated by two el ectron excitations. According to Casida and co-workers ${ }^{145}$ the spin contamination problem arises from multiple coupling of states with a different spin. This creates fictitious quartets or two doublet excited states which mix. Note that $S^{2}$ for excited state with multiplicity equal to four (quartet) is 3.75 and such high values were not found. 
Table 4.10: M06L predicted LMCT transitions with corresponding oscillator strength (f) for the $\left[\mathrm{Cu}(\mathrm{IAC})_{2}\right]$ complex in vacuum, methanol and dichloromethane. $\lambda$ is the wavelength, EE is the excitation energy, cx stands for carboxylate, im for imidazole and et for ethyl group.

\begin{tabular}{|c|c|c|c|}
\hline \multicolumn{4}{|c|}{ VACUUM } \\
\hline Type $^{\text {a }}$ & $\lambda(\mathrm{nm})$ & $\mathrm{EE}(\mathrm{eV})$ & $f \times 10^{-2}$ \\
\hline $\mathrm{n}(\mathrm{O}-\mathrm{cx}) \rightarrow \mathrm{d}_{\mathrm{Cu}^{2+}}$ & 587 & 2.11 & 2.28 \\
\hline$\sigma(\mathrm{O}-\mathrm{cx}) \rightarrow \mathrm{d}_{\mathrm{Cu}^{2+}}$ & 429 & 3.07 & 2.93 \\
\hline $\mathrm{n} / \sigma(\mathrm{O}-\mathrm{cx}) \rightarrow \mathrm{d}_{\mathrm{Cu}^{2+}}$ & 413 & 3.11 & 2.30 \\
\hline \multicolumn{4}{|c|}{$\mathrm{CH}_{3} \mathrm{OH}$} \\
\hline $\mathrm{n}(\mathrm{O}-\mathrm{cx}) \rightarrow \mathrm{d}_{\mathrm{Cu}^{2+}}$ & 587 & 2.11 & 2.28 \\
\hline $\mathrm{n}(\mathrm{O}-\mathrm{cx}) \rightarrow \mathrm{d}_{\mathrm{Cu}^{2+}}^{\mathrm{b}}$ & 439 & 2.82 & 3.57 \\
\hline$\sigma(\mathrm{O}-\mathrm{cx}) \rightarrow \mathrm{d}_{\mathrm{Cu}^{2+}}$ & 375 & 3.31 & 12.0 \\
\hline $\mathrm{Cu}-\mathrm{p} / \mathrm{n}(\mathrm{im}) \rightarrow \mathrm{d}_{\mathrm{Cu}^{2+}}$ & 238 & 5.21 & 6.64 \\
\hline \multicolumn{4}{|c|}{$\mathrm{CH}_{2} \mathrm{Cl}_{2}$} \\
\hline $\mathrm{n}(\mathrm{O}-\mathrm{cx}) \rightarrow \mathrm{d}_{\mathrm{Cu}^{2+}}^{\mathrm{b}}$ & 570 & 2.18 & 1.96 \\
\hline $\mathrm{n}(\mathrm{O}-\mathrm{cx}) / \pi(\mathrm{im}) \rightarrow \mathrm{d}_{\mathrm{Cu}^{2+}}$ & 439 & 2.83 & 3.78 \\
\hline$\sigma(\mathrm{O}-\mathrm{cx}) \rightarrow \mathrm{d}_{\mathrm{Cu}^{2+}}$ & 379 & 3.28 & 11.8 \\
\hline $\mathrm{Cu}-\mathrm{p} / \mathrm{n}(\mathrm{im}) \rightarrow \mathrm{d}_{\mathrm{Cu}^{2+}}$ & 5.29 & 234 & 16.3 \\
\hline
\end{tabular}

Also for adiabatic TDDFT (a single-electron cal culations) the spin-flip configurations are impossible, because such event requires two electrons to be excited. ${ }^{132}$ Therefore, excited states and associated transitions with $S^{2} \quad 1$ are possibly artificial or have an nonphysically high spin contamination. 


\subsection{LRC-TDDFT Results and Discussion}

The set of calculations for both the $\mathrm{Cu}$ (II) complexes considered here was performed using CAM-B3LYP* with following values for $\mathrm{y}: 0.20,0.25,0.33$, and 0.40 ; the aim was done to study the effect of $y$ on the $d-d$ transitions. In addition, the effect of the fraction of the exact exchange (defined by $\alpha$ in the short-range, and $\alpha+\beta$ in the long-range) was monitored by changing $\alpha$ from the standard $19 \%$ to $10 \%, 15 \%$, and $25 \%$ and using the same value of the range-separation parameter. Furthermore, other LRC functionals were employed: where

$\omega B 97 X$ contains about $16 \%$ of the exact exchange in short-range (al so noted as $E_{x}^{(S R-H F)}$, see Chapter 2 section 2.2.2.1) and LC- $\omega$ PBE with its $0 \%$ of exact exchange. Results calculated with these functional s will allow to compare the performance of two different LRC hybrid functional s with a pure LRC functional as well as with standard $x c$ functional s. Note that in our notation $\omega=\gamma$, where $\omega$ was kept to avoid changing the original convention on the names for these functionals.

In Fig. 4.5 the UV-Vis spectra of the $\left[\mathrm{Cu}(\mathrm{IA})_{2}\right]$ complex are compared to show the contrast between uncorrected B3LYP, the long-range corrected CAM-B3LYP and asymptotically corrected CAM-B3LYP*. The CAM-B3LYP, provides al ready some improvements to the computed spectrum; mainly the first d-d transition is found at $548 \mathrm{~nm}(2.26 \mathrm{eV})$ whereas with B3LYP the first $d-d$ transition is at $518 \mathrm{~nm}(2.39 \mathrm{eV})$. Furthermore the LMCT transition assigned as $\sigma(\mathrm{O}-\mathrm{cx}) \rightarrow \mathrm{d}_{\mathrm{Cu}^{2+}}$ is blue shifted from $328 \mathrm{~nm}(3.78 \mathrm{eV})$ to $280 \mathrm{~nm}$ $(4.43 \mathrm{eV})$ with about $35 \%$ increase in intensity. This transition was found in the filtered spectrum at $254 \mathrm{~nm}(4.88 \mathrm{eV})$ and it is one of the most intense LMCT transitions (the other two are $\pi(\mathrm{im}) \rightarrow \mathrm{d}_{\mathrm{Cu}^{2+}}$ at $290 \mathrm{~nm}(4.28 \mathrm{eV})$ and $\mathrm{n}(\mathrm{im}) \rightarrow \mathrm{d}_{\mathrm{Cu}^{2+}}$ at $\left.228 \mathrm{~nm}(5.44 \mathrm{eV})\right) .{ }^{123}$ CAM-B3LYP* seems to further improve the theoretical spectrum, predicting the first $d-d$ at $554 \mathrm{~nm}(2.24 \mathrm{eV})$ with an error of $0.38 \mathrm{eV}$ with respect to the original data (i.e., $666 \mathrm{~nm}$ or $1.86 \mathrm{eV}) .^{123}$ 

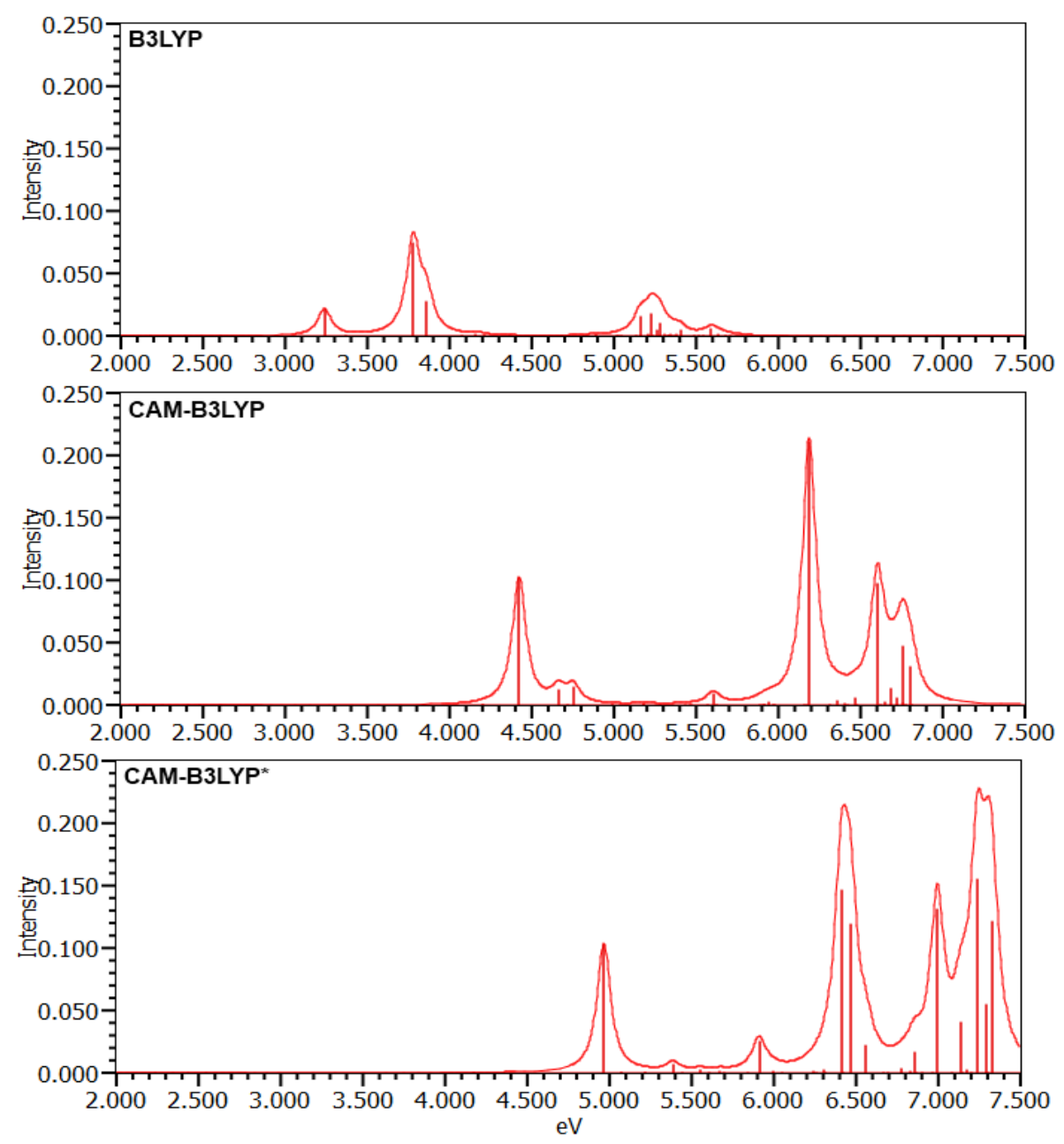

Figure 4.5: UV-Vis spectra of the $\left[\mathrm{Cu}(\mathrm{IA})_{2}\right]$ complex in vacuum cal culated with B3LYP, CAM-B3LYP and CAM-B3LYP* Intensity is given in terms of the oscillator strength, and is dimensionless.

The $\sigma(\mathrm{O}-\mathrm{cx}) \rightarrow \mathrm{d}_{\mathrm{Cu}^{2+}}$ found at $250 \mathrm{~nm}(4.97 \mathrm{eV})$ is even more blue shifted and an additional absorbtion band appeared at $189 \mathrm{~nm}(6.57 \mathrm{eV})$ which may be interpreted as $\pi(\mathrm{im}) \rightarrow$ $\mathrm{d}_{\mathrm{Cu}^{2+}}$. Unfortunately this peak is both spin contaminated and mixed in character, hence the quantitative comparison with experiment is fairly poor. Although in this range of the spectrum some Rydberg states may occur, it is not expected for these high-laying excitations to invol ve mixed Rydberg-val ance transitions as it has been shown that the asymptotic correction approach evades this problem. ${ }^{146}$ 
A few lowest vertical excitations from the ground-state energy level (arbitrarily assigned as $0 \mathrm{eV}$ ) are schematically illustrated in Fig. 4.6 and Fig. 4.7 to demonstrate changes upon $L R C$ and asymptotic correction for $d-d$ transitions. The notation $X_{n}^{d(b)}$ used in both the figures is applied to distinguish between excited states computed with a given functional. In our notation, $\mathrm{n}$ is the order of the excited state, $\mathrm{d}$ stands for dark (optical ly inactive) state, $\mathrm{b}$ for bright (optically active) state, A, B and C for B3LYP, CAM-B3LYPand CAM-B3LYP*, respectively.

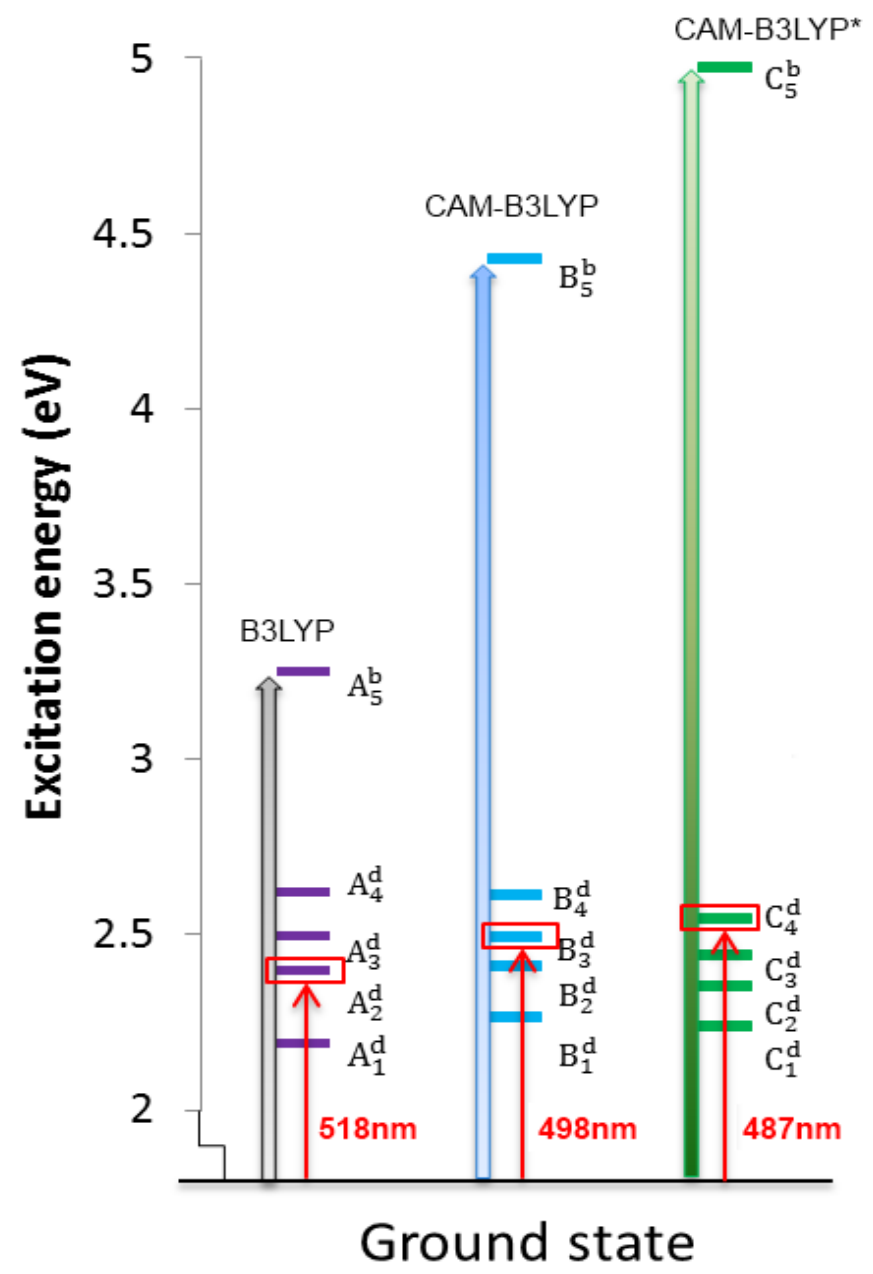

Figure 4.6: Lowest excited states of the $\left[\mathrm{Cu}(\mathrm{IA})_{2}\right]$ complex. Influence of the $L R$ and asymptotic correction on $\beta$-spin $d$-d transition from HOMO-14 to LUMO in vacuum obtained with B3LYP, CAM-B3LYP, and CAM-B3LYP*. 


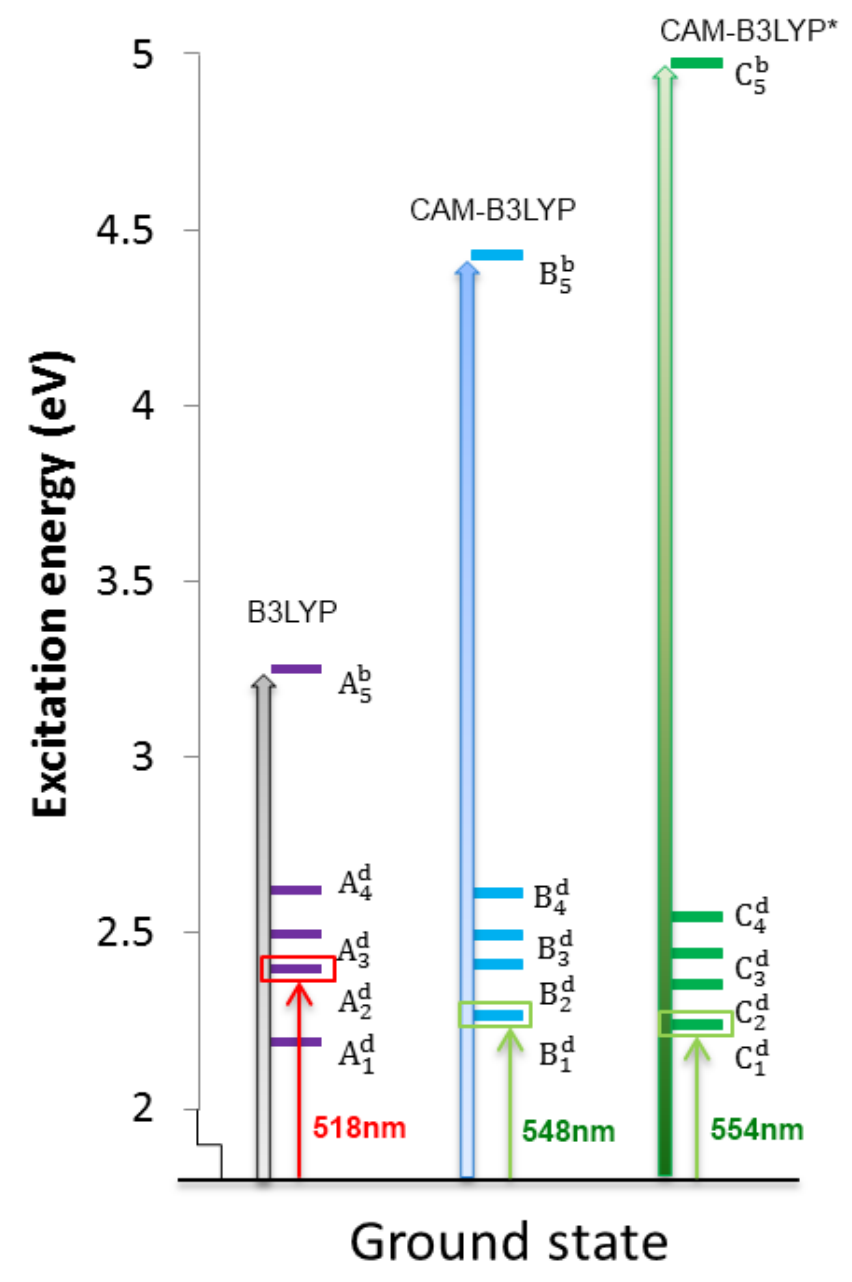

Figure 4.7: Lowest excited states of the $\left[\mathrm{Cu}(\mathrm{IA})_{2}\right]$ complex. Influence of the $L R$ and asymptotic correction on $\beta$-spin d-d transition from HOMO-10 to LUMO in vacuum obtained with B3LYP, CAM-B3LYP, and CAM-B3LYP*.

The thick arrows represent the so-called optical gaps (i.e., the energy difference between the ground-state and the first optical ly active excited-state) whereas the thin arrows correspond to the excitation energies (defined as $\Delta E=E_{e s}-E_{g s}$, where $E_{e s}$ is the energy of a given excited-state and $E_{g s}$ is the energy of the ground-state) of one of the predicted d-d transitions. The first excited state computed with $B 3 L Y P\left(A_{1}^{d}\right)$ is a dark state that can be described as a mixed transition composed of excitations $\mathrm{O}-\mathrm{p}$ (carboxylate) $\rightarrow \mathrm{d}_{\mathrm{Cu}^{2+}}$ and $\mathrm{d} \rightarrow \mathrm{d}$. The $A_{2}^{d}$ state involves single $\beta$-spin electron excitation from HOMO-14 to LUMO; this can 
be assigned as the first "pure" $d$-d transition. When LR correction is added the energy is upward shifted by about $0.1 \mathrm{eV}$, bringing the $B_{3}^{d}$ state at about $2.5 \mathrm{eV}$. The asymptotic correction (AC) shifts up the $B_{3}^{d}$ state by a small amount of energy $(0.05 \mathrm{eV})$, hence at $A C$ CAM-B3LYP (CAM-B3LYP*) level of theory this state becomes the forth $\left(\mathrm{C}_{4}^{d}\right)$ in terms of the energy level ranking. Although the HOMO-14/LUMO gap does not correspond to any computed excitation energies, an intuitive prediction that excitation from a deeper occupied energy level should cost a greater input of energy is confirmed by the fully corrected CAM-B3LYP* functional. In other words, the optical gap produced by B3LYP is too small, which causes the excitation energies to be lower than those obtained within the long-range asymptotically corrected scheme. Fig. 4.7 shows that when the optical gap opens up new excited states can be found, for instance the $B_{1}^{d}$ and $C_{1}^{d}$. The population analysis allows assigning dominating transition as $\mathrm{d}$-d. Note that for both these excited states the most significant transition is from HOMO-11 $\beta$ to LUMO $\beta$; however in case of CAM-B3LYP* both electron donor and acceptor are contaminated by ligand-based orbitals. The rangeseparation parameter $(y=0.33)$ utilized by CAM-B3LYP was determined empirically, ${ }^{147}$ and the fraction of exact exchange in the short-range was kept the same as for B3LYP ( $\alpha=0.19$ ), which suggested that for this functional the best choice for $\beta$ is $0.45 .{ }^{103} \mathrm{De}$ spite the LR correction to B3LYP provided by CAM, the correct asymptotic behaviour of the xc potential in the long-range was not exactly restored due to the fact that a standard $x c$ functional is fully corrected when $\alpha+\beta=1 .{ }^{111}$ For this reason CAM-B3LYP* is utilized, but sine the best results for $\gamma=0.33$ were obtained with $\alpha+\beta=0.65$ the are no evidences that this val ue of $y$ will work as well with $\alpha+\beta=1$. For example, for LC- $\omega P B E 0$ functional the best value for $\gamma$ was found to be 0.20 (at least for ground-sate properties). ${ }^{111}$

The next sections will be dedicated to shead some light onto how the electronic transitions will be affected by the change of $y$ and $\alpha$. Of particular interest for this project are positions and characters of the lowest excited states because it is expected that $d-d$ transitions will occur between the ground-state and the excited states laying at about $2 \mathrm{eV}$ above. 


\subsubsection{TheEffect of the Range-Separation Parameter and the Fraction of Exact Exchange on Electronic Transitions}

The important finding emerging from computations at CAM-B3LYP* level of theory is that for some of the predicted d-d transitions the excitation energy changed using different values of $y$ whereas for other $d-d$ transitions such change did not occur (see Table 4.11). Therefore, some computed wavelengths are red shifted with respect to val ues obtained for CAM-B3LYP* using a smaller $\mathrm{Y}$. Why do we observe these differences in transition for $\mathrm{Cu}(\mathrm{II}) \mathrm{d}$-manifold orbitals? In order to to answer this question the population analysis was analyzed to address the effect of $\mathrm{y}$ on the character and the energy of molecular orbitals involved in a given electronic transition assigned as $d-d$. Also the performance of CAMB3LYP* with the" best" val ue of the range-separation parameter will be re-examined while including a varying amount of the exact exchange in the short range (for more details see section 4.2.2).

Among all the LRC functionals explored in this work the best agreement with absorption bands assigned as $d-d$ transition at $666 \mathrm{~nm}^{123}$ is obtained with CAM-B3LYP* (using $Y=0.20, y=0.25$ and $y=0.40$ ) and $\omega B 97 X$, for which the excitation energy was overestimated by about $0.3-0.4 \mathrm{eV}$. Somewhat surprising is such a good agreement of CAM$B 3 L Y P^{*}$ functional with $y=0.40$ due to the fact that the first excited state can be described as mixed d-d. Moreover CAM-B3LYP* $(\gamma=0.40)$ introduced greater error to LMCT transitions than with other values of $y$ as well as with $\omega B 97 X$ and LC- $\omega P B E$ functionals. Considering both types of transitions (metal-based and ligand-based) the best description for the $\left[\mathrm{Cu}(\mathrm{IA})_{2}\right]$ complex spectrum was achieved with CAM-B3LYP* $(Y=0.25)$. 


\subsubsection{The Range-Separation Par ameter}

Table 4.11 shows cal culated excitation energies and wavel engths of $d-d$ transitions for the $\left[\mathrm{Cu}(\mathrm{IA})_{2}\right]$ complex in vacuum. The oscillator strengths have been omitted due to the fact that all gas-phase $d-d$ transitions for the square-planar geometry are symmetry forbidden. For the LRC functionals employed herein the first four excited states involve dominating molecular orbital pair with varying percentage of $\mathrm{Cu}-\mathrm{d}$ character. For example, the first excited state computed with CAM-B3LYP* using $Y=0.20$ is dominated by $\beta$-spin HOMO-11 and LUMO pair, where the former contains about 55\% of $\mathrm{Cu}-\mathrm{d}$ and the latter $66 \%$. This allows us to assign this transition as $\mathrm{d}-\mathrm{d}$. All active transitions (mainly LMCT) of the $\left[\mathrm{Cu}(\mathrm{IA})_{2}\right]$ complex predicted with LRC-TDDFT are shown in Fig. 4.8 The spectral line-shapes are reported as indicated before (i.e., lines are broadened with $0.05 \mathrm{eV}$ HalfWidth Lorentzian). Note that these absorption bands are found within the spectral range 2-7.5 eV and for this reason the UV-Vis spectra were" zoomed-in" to illustrate more clearly the peaks of interest.

CAM-B3LYP, CAM-B3LYP* with $\gamma=0.20$ and $\gamma=0.25$ as well as $\omega B 97 X$ are producing two excited states which can be described by the same dominating MO pair involved in a given electronic transition. However, one of these excited states has a slightly smaller contribution from "shared" MO pair and at least one another significant donor-acceptor combination (see footnotes in the Table 4.11). For CAM-B3LYP and CAM-B3LYP* $(Y=0.25)$ this second most important contribution to the third excited state al so involves MOs with $\mathrm{Cu}$-d character, where the electron donor is one of the energetically deeper occupied dorbital s. For CAM-B3LYP* $(\gamma=0.20)$ the second excited state includes similarly contributing MO pairs, but in this case the second one can be assigned as a mixed transition. In fact, the HOMO-7 is composed of $\mathrm{Cu}-\mathrm{d}(15 \%)$ and $\mathrm{O}-\mathrm{p}(26 \%)$ from each of the carbonyl of the two carboxyl groups. For $\omega B 97 X$ the first and the second excited states are both dominated by Cu-d character. 

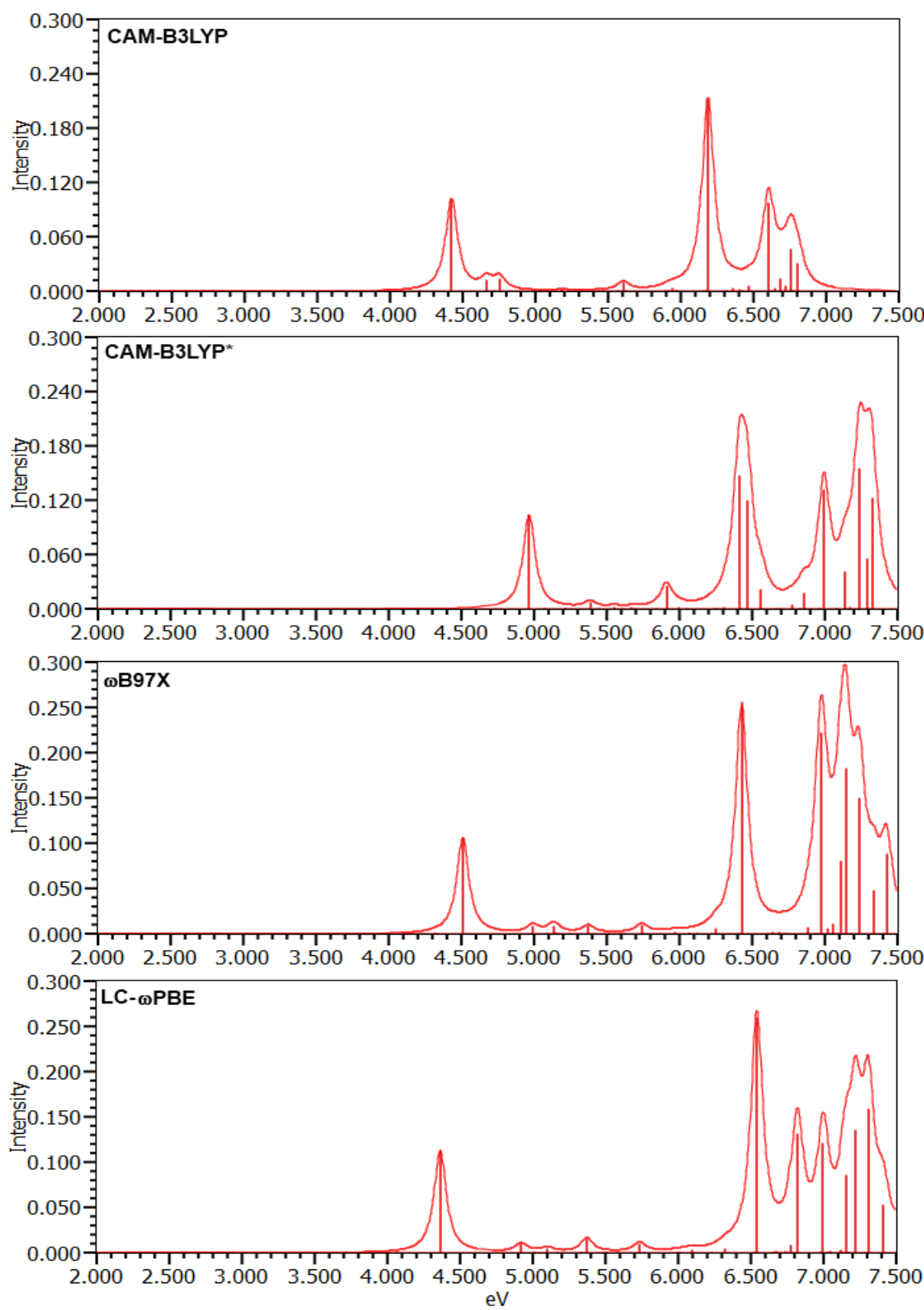

Figure 4.8: Comparison of the LRC-TDDFT absorption spectra of the $\left[\mathrm{Cu}(\mathrm{IA})_{2}\right]$ complex in vacuum calculated with CAM-B3LYP, CAM-B3LYP*, $\omega B 97 X$ and LC- $\omega P B E$. Intensity is given in terms of the oscillator strength, and is dimensionless. 


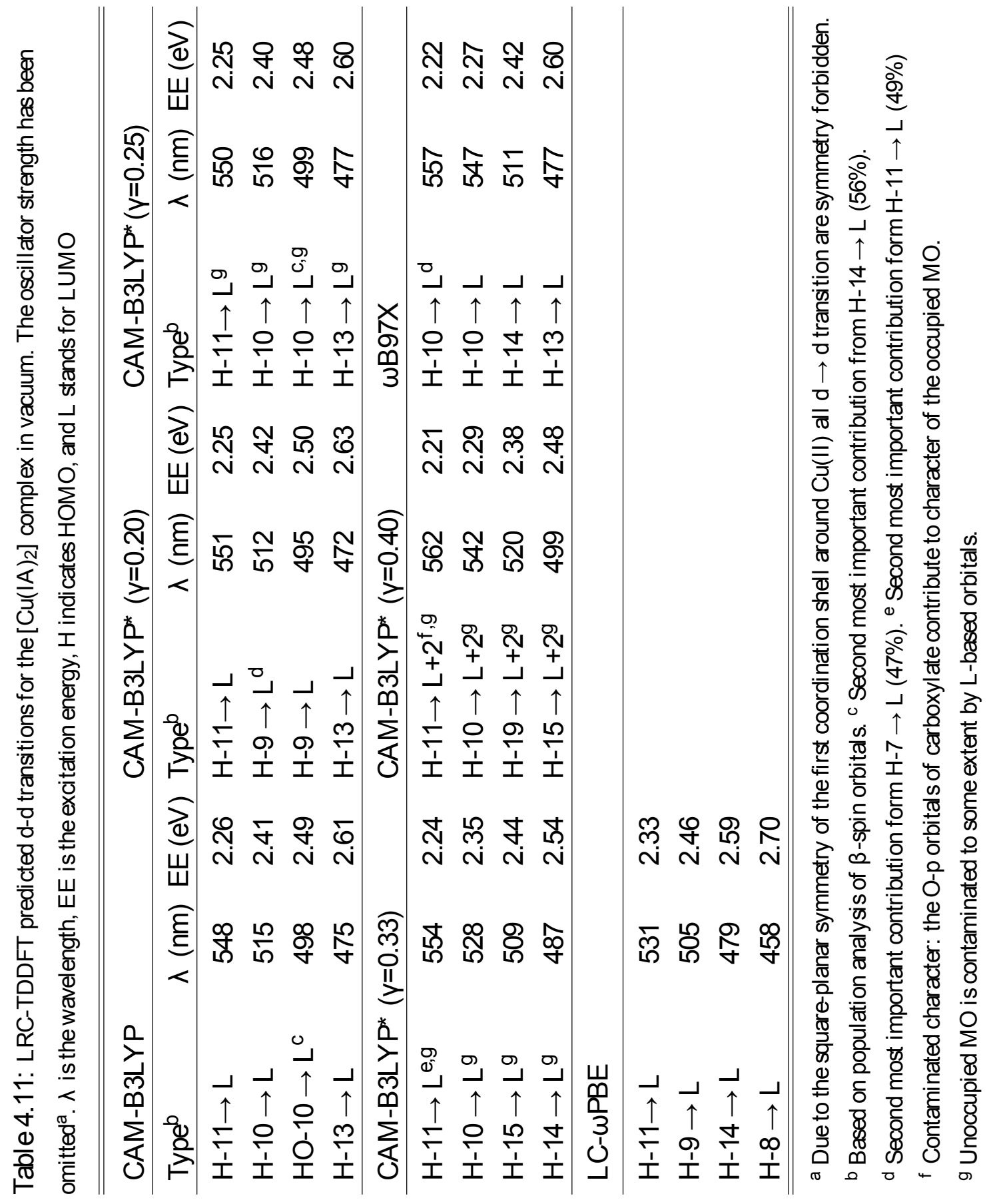


The energy shift for these excited states, i.e., from the third to second and to first, can be associated by using different parameters affecting occupied/unoccupied energy levels and therefore the spectra. Perhaps the most surprising is the change between wB97X and CAM-B3LYP*, despite being very similarly parameterized. The percentage of $\mathrm{Cu}-\mathrm{d}$ and the $\beta$-spin orbital energies are given in Table 4.12. CAM-B3LYP is omitted due to the lack of a proper asymptotic behaviour for its xc potential, i.e., $\alpha+\beta=0.65$.

Molecular orbitals involved in the same $d-d$ transitions which have been found for almost all the LRC functionals are discussed in the following. Observations for CAM-B3LYP* with various val ues of $y$ are:

† For increasing values of $\mathrm{y}$ the percentage of Cu-d for occupied orbitals decreases, e.g., HOMO-11 with $y=0.20$ has $59 \%$, which decreases to $53 \%$ with $y=0.25$, to $42 \%$ for $\gamma=0.33$, and to $33 \%$ for $\gamma=0.40$.Furthermore, contamination from ligandbased orbitals increases.

† As the value of $y$ increases the orbital energy of occupied orbitals becomes more negative: from $-11.7 \mathrm{eV}$ to $-13.5 \mathrm{eV}$.

† For increasing value of $\mathrm{y}$ the percentage of $\mathrm{Cu}$ - $\mathrm{d}$ for unoccupied orbitals decreases. Note for the $y=0.40$ LUMO $\beta$ contains only $13 \%$ of Cu-d, hence LUMO $2 \beta(55 \%$ $\mathrm{Cu}-\mathrm{d})$ is the actual acceptor of the electron from the other $\mathrm{d}$-orbital of $\mathrm{Cu}(\mathrm{II})$.

† For increasing vale of $y$ the energy of unoccupied orbitals becomes more positive: from $-0.4 \mathrm{eV}$ to $1.1 \mathrm{eV}$. 


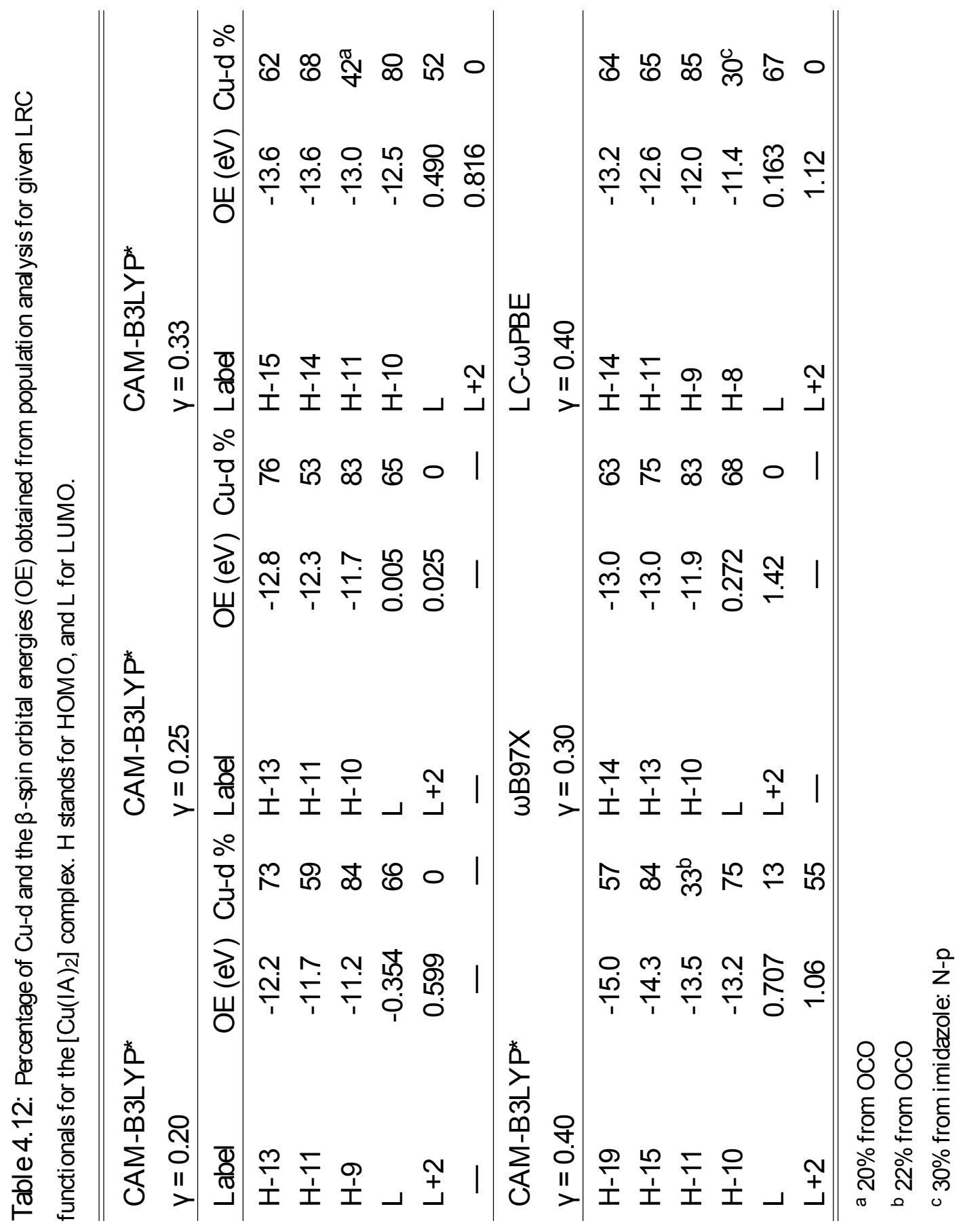


The following comments correl ate the position of the $d$ - $d$ transition from $\beta$-spin HOMO-11 $\rightarrow$ LUMO/LUMO+2 in the UV-Vis spectrum:

$\dagger$ No shift is observed when $\gamma$ increase by 0.05 bohr $^{-1}$ (going from $\gamma=0.20$ to $\gamma=0.25$ ). The difference between percentage of $\mathrm{Cu}-\mathrm{d}$ in HOMO-11 and of $\mathrm{Cu}-\mathrm{d}$ in LUMO is $12 \%$ (this change will be indicated as $\Delta \mathrm{Cu}-\mathrm{d} \%$ ). In other words, these two MO have similar character. For $\mathrm{y}=0.20 \Delta \mathrm{Cu}-\mathrm{d} \%$ is even smaller: $7 \%$.

† The HOMO-11 energy lowers by $0.60 \mathrm{eV}$ whereas LUMO energy increases by $0.35 \mathrm{eV}$ with respect to val ues cal cul ated with $y=0.20$. Hence the HOMO-11/LUMO energy gap $\left(\Delta \mathrm{E}_{\mathrm{H}_{11}}^{\mathrm{L}}\right)$ is increased by al most $1 \mathrm{eV}$; this change will be denoted as $\Delta \Delta_{25}$ and it is cal culated from $\left.\Delta \Delta_{25}=\Delta \mathrm{E}_{\mathrm{H}_{11}}^{\mathrm{L}}\left(\mathrm{Y}_{0.25}\right)-\Delta \mathrm{E}_{\mathrm{H}_{11}}^{\mathrm{L}}\left(\mathrm{Y}_{0.20}\right)\right)$.

† A very small red shift occurs if $y$ value is increased by additional 0.08 bohr $^{-1}$ (from 0.25 to 0.33 ). The wavelength of this $\mathrm{d}$ - $d$ transition changes from $550 \mathrm{~nm}$ to $554 \mathrm{~nm}$. Again the character of the involved MOs is similar ( $\Delta \mathrm{Cu}-\mathrm{d} \%$ is $10 \%)$. From an energetic stand point the HOMO-11 lowers by $0.70 \mathrm{eV}$ and the LUMO increases by $0.48 \mathrm{eV}$ with respect to val ues obtained with $\mathrm{y}=0.25 . \Delta \Delta_{33}=1.2 \mathrm{eV}$ with respect to the $\Delta \mathrm{E}_{\mathrm{H}_{11}}^{\mathrm{L}}$ obtained with $\mathrm{y}=0.25$. These val ues for $\Delta \Delta_{25}$ and $\Delta \Delta_{33}$ reflect an overal small change from smaller to bigger values of $\mathrm{Y}(0.2 \mathrm{eV})$.

† When $y$ is increased to 0.40 dramatic changes in the LUMO arise, and the d-d transition are then described by HOMO-11 and LUMO+2. The wavelength red shifts from $554 \mathrm{~nm}$ to $562 \mathrm{~nm}$. The character of this MO pair in terms of percentage of $\mathrm{Cu}-\mathrm{d}$ differs now by $22 \%$. The energy of HOMO- 11 decreases by $0.5 \mathrm{eV}$ while the energy difference between $L U M O_{\gamma}=0.33$ and $L U M O+2_{\gamma}=0.40$ is $0.6 \mathrm{eV}$. This would indicate a smaller change of $\Delta \mathrm{E}_{\mathrm{H}_{11}}^{\mathrm{L}}$ from that cal culated with $\mathrm{Y}=0.33$. However, in this situation, $\Delta \mathrm{E}_{\mathrm{H}_{11}}^{\mathrm{L}}$ becomes $\Delta \mathrm{E}_{\mathrm{H}_{11}}^{\mathrm{L}+2}$ and the effect of increasing $Y$ value is not as explicit as before. Comparisons can be done between a hypothetical $\Delta \mathrm{E}_{\mathrm{H}_{11}}^{\mathrm{L}(\mathrm{y}=0.40)}$ 
and the value obtained with $y=0.33$ (which is, according to previous convention, denoted as $\Delta \Delta_{40}$ ), and the actual $\Delta \mathrm{E}_{\mathrm{H}_{11}}^{\mathrm{L}+2}$ with respect to the same value (denoted as $\left.\Delta \Delta_{40}\right)$. Following this scheme $\Delta \Delta_{40}=0.7 \mathrm{eV}$ and $\Delta \Delta_{40}=1 \mathrm{eV}\left(\Delta \Delta_{0.40}=\Delta \mathrm{E}_{\mathrm{H}_{11}}^{\mathrm{L}+2}(\mathrm{\gamma}=\right.$ $\left.0.40)-\Delta \mathrm{E}_{\mathrm{H}_{11}}^{\mathrm{L}}(\mathrm{Y}=0.33)\right)$ therefore indirectly, the overall change is slightly bigger $(0.3 \mathrm{eV})$ than the change associated with $\mathrm{y}$ increasing from 0.25 to 0.33 .

Note that for a simple complex such as $\left[\mathrm{CuCl}_{4}\right]^{2-}$ it has been shown that with a larger amount of metal ( $60 \%$ ) on the highest occupied molecular orbitals, the d-manifold is more destabilized causing LF (d-d) transitions to shift to lower energies, and LMCT transitions to higher energies. ${ }^{148}$ Also, tuned long-range corrected hybrid functionals stretch the HOMOLUMO (HL) gap by including the so-called derivative discontinuity. ${ }^{105}$ As a consequence the fundamental gap equals the HL gap (see for example work of Cohen et al. ${ }^{42}$ ).

Resultsfor the investigation of the $\left[\mathrm{Cu}(\mathrm{IA})_{2}\right]$ complex showed that $\Delta \mathrm{E}_{\mathrm{H}_{11}}$ is indeed widened by applying the LRC scheme but the eigenvalues of orbital sused to compute this energy gap do not reproduce the excitation energy for the $d_{y z} \rightarrow d_{x^{2}-y^{2}}$ transition. Furthermore, it appears that a somewhat opposite effect than that reported for $\left[\mathrm{CuCl}_{4}\right]^{2-}$ is observed. Mainly, the $d-d$ transition with the lowest excitation energy, for calculations with CAMB3LYP* $(y=0.40)$, involves MOs with the smallest percentage of $\mathrm{Cu}-\mathrm{d}$. Perhaps this disagreement is caused by the involvement of electron excitation from molecular orbitals laying much below the HOMO energy level or by the fact that neither of the CAM-B3LYP* variants tested herein is actually properly tuned. Furthermore, even though the following trend can be observed

$$
\begin{gathered}
\Delta \mathrm{Cu}-\mathrm{d} \%(\mathrm{\gamma}=0.20) \approx \Delta \mathrm{Cu}-\mathrm{d} \%(\mathrm{\gamma}=0.25) \approx \Delta \mathrm{Cu}-\mathrm{d} \%(\mathrm{\gamma}=0.33)<\Delta \mathrm{Cu}-\mathrm{d} \%(\mathrm{\gamma}=0.40) \\
\text { and } \lambda_{20} \approx \lambda_{25} \approx \lambda_{33}<\lambda_{40}
\end{gathered}
$$

the $\beta$-spin HOMO-9/HOMO-10 $\rightarrow$ LUMO/LUMO+2 $\left(d_{z^{2}} \rightarrow d_{x^{2}-y^{2}}\right)$ transition, which al so was predicted by all the LRC-TDDFT functionals considered herein, behaves differently 
than $d_{y z} \rightarrow d_{x^{2}-y^{2}}$. The $d-d$ transition from $d_{z^{2}}$ is more affected by change in $y$ than the $d-d$ transition from $d_{y z}$. In this case there is no simple correlation between change in procentage of $\mathrm{Cu}$ and the wavelength shift:

$$
\begin{gathered}
\Delta \mathrm{Cu}-\mathrm{d} \%(\mathrm{\gamma}=0.20)=\Delta \mathrm{Cu}-\mathrm{d} \%(\mathrm{\gamma}=0.25) \quad \Delta \mathrm{Cu}-\mathrm{d} \%(\mathrm{\gamma}=0.33)>\mathrm{y} \mathrm{Cu}-\mathrm{d} \%(\mathrm{\gamma}=0.40) \\
\text { and } \lambda_{20}<<\lambda_{25}<\lambda_{33} \approx \lambda_{40} .
\end{gathered}
$$

This implies that an explanation for the observed shifts in the position of the d-d transitions is still eluding; in fact, the lack of such shifts associated with a small $\Delta$ Cu-d\% (i.e, similar character of both the orbitals involved in transition), which would not depend upon $y$ cannot be justified yet.

Is $y$ the only aspect that needs to be considered? To answer this question results from LC- $\omega$ PBE and $\omega$ B97X cal culations will be first anal yzed.

For LC-wPBE (LRC "pure" GGA functional, i.e., no SR HF exchange considered) the wavel ength of the $d-d$ transition: HOMO-11 $\beta \rightarrow L U M O \beta$ is $531 \mathrm{~nm}$, and red shifts with respect to the CAM-B3LYP* with $y=0.40(562 \mathrm{~nm})$. Nevertheless the character of MOs in terms of $\%$ of $\mathrm{Cu}-\mathrm{d}$ obtained in LC- $\omega \mathrm{PBE}$ is al most the same ( $\triangle \mathrm{Cu}-\mathrm{d}=2 \%$ ). Moreover, again with metallic character on HOMO-11 $\beta$ cal culated with this functional the LF transition is energetically pushed to higher energy; this behaviour opposite to what was observed for the $\left[\mathrm{CuCl}_{4}\right]^{2-}$ complex. ${ }^{148}$ Thus with respect to the experimental data ${ }^{123}(666 \mathrm{~nm})$ this transition is more blue shifted than when computed with CAM-B3LYP* $(Y=0.40)$. In the case of the HOMO-9 $\rightarrow$ LUMO $\beta$ transition obtained with LC- $\omega$ PBE $(505 \mathrm{~nm})$ the $\Delta \mathrm{Cu}-\mathrm{d} \%$ is $18 \%$ whereas the same transition (in terms of character) computed with CAM$B 3 L Y P^{*}(Y=0.40)$ is found at $528 \mathrm{~nm}$ where $\Delta C u-d \%=28$. In spite of using a different LRC functionals these observations demonstrate once more a different treatment of $d_{z^{2}} v s$. $d_{y z}$. Note that for LC- $\omega P B E$ the $\beta$-spin MOs involved in the two predicted d-d transitions described above have the following characters: $d_{y z}(H O M O-11), d_{z^{2}}(H O M O-9)$ and $d_{x^{2}-y^{2}}$ 
(LUMO). With $y$ having the same value, the reason for such shifts can be due to either a change in the LRC xc functional itself or in including fraction of HF exchange in the short-range.

Results from $\omega B$ 97X, helps to establish that the choice of $y$ is not the only factor; more likely both the fraction of SR HF exchange (noted as $\alpha$ ) and $y$ are equal ly influential. Unfortunately the only common $d-d$ transition found with $\omega B$ 97X is HOMO-10 $\rightarrow$ LUMO $\beta$ $\left(d_{z^{2}} \rightarrow d_{x^{2}-y^{2}}\right.$, which was predicted at $547 \mathrm{~nm}$ with $\Delta C u-d=15 \%$. This shift with respect to results obtained with CAM-B3LYP* $\left(\lambda_{\mathrm{d}_{z^{2}} / \mathrm{d}_{\mathrm{x}^{2}-\mathrm{y}^{2}}}=528 \mathrm{~nm}\right)$ is somewhat surprising as $\omega B 97 X$ functional is very similarly parameterized, i.e., $y=0.30, \alpha=0.16$. $\Delta$ Cu-d\% for this MO pair in CAM-B3LYP* is $13 \%$ higher than in $\omega B$ 97X. Consequently, this can be an argument in favour of the hypothesis: that LF transitions are described more accurately with a small $\Delta \mathrm{Cu}$-d\%. However, taking into account that in this investigation main three factors are involves (functional, the value of the range-separation parameter, and the various amounts of the SR HF exchange) and they all influence the final position of the predicted d-d transitions, the evidences presented so far do not al low to confirm any unique answer.

In addition it appears that calculated spectra of the $\left[\mathrm{Cu}(\mathrm{IA})_{2}\right]$ with the variants of $\mathrm{CAM}$ $B 3 L Y P^{*}$ tested herein might suffer from the so-called delocal ization error. ${ }^{111}$ Therefore the following question may arise: will the minimization of this error hel p in treating differently $\sigma$-type and $\pi$-type donors?

Some insights necessary to address this issue follow. As mentioned before for the considered complexes electron donating MOs involved in $\mathrm{d}$-d transitions are energetically deeper than HOMO, and the d-manifold of a metal center in the square planar field can be separated into $\sigma$-type $\left(d_{z^{2}}, d_{x^{2}-y^{2}}\right)$ and $\pi$-type $\left(d_{x z}, d_{y z}\right.$ and $\left.d_{x y}\right){ }^{149}$ Both characteristics of Cu-d orbitals will become critical in consideration of possible reasons behind the occurring changes in resulting spectra. The technique called Ionization Potential (IP) 
tuning ${ }^{150}$ is often used to find the best $\mathrm{Y}$ and it is based on the Janak's theorem that $I P(N)=-\varepsilon_{\text {HOMO }}(N) .{ }^{151}$ Mainly, for a given standard $x c$ functional and a given system the appropriate range-separation parameter should minimize $\mathrm{J}^{2}(\mathrm{Y})$ defined as

$$
J^{2}(Y) \equiv\left[\varepsilon_{H O M O}(N ; \gamma)+I P(N ; \gamma)\right]^{2}
$$

where $\varepsilon_{\text {HOMO }}$ is the energy of the highest occupied molecular orbital. IP is cal culated as the energy difference between the ground state of the cation and the neutral species:

$$
I P(N)=E(N-1)-E(N)
$$

Note that a more rigorous version of $\mathrm{J}^{2}(\mathrm{\gamma})$ includes al so orbital energy of the lowest unoccupied molecular orbital and electron affinity. 152,153 The results of the investigation for those values of the range-separation parameter that minimize $\mathrm{J}^{2}(\mathrm{y})$ are shown in $\mathrm{Ta}$ ble 4.13.

The vertical IP is obtained as $\triangle \mathrm{SCF}$, i.e., by subtracting the Self-Consistent Field (SCF) DFT energy of negatively charged and neural complex of $\mathrm{Cu}(\mathrm{II})$. Note that while the geometry of the $\left[\mathrm{Cu}(\mathrm{IA})_{2}\right]^{-}$species is not reoptimized for the multiplicity changes from doublet to singlet, thus the orbital energy of $\mathrm{HOMO} \alpha$ of the neutral complex is used to compute $\mathrm{J}^{2}(\mathrm{Y})$.

Table 4.13: Cal culated vertical IP(N) of the neutral $\left[\mathrm{Cu}(\mathrm{IA})_{2}\right]$, SCF-DFT energies of neutral complex and its cation, orbital energies of $\mathrm{HOMO}(\mathrm{N})$ and $\mathrm{J}^{2}(\mathrm{Y})$ values.

\begin{tabular}{cccccc}
\hline \hline CAM-B3LYP* & & & & & \\
$\mathrm{Y}$ & $\varepsilon_{\text {HOMO }}(\mathrm{eV})$ & $\mathrm{E}(\mathrm{N})(\mathrm{eV})$ & $\mathrm{E}(\mathrm{N}-1)(\mathrm{eV})$ & $\mathrm{IP}(\mathrm{eV})$ & $\mathrm{J}^{2}(\mathrm{eV})^{2}$ \\
\hline 0.20 & -8.39 & -30030.72 & -30022.25 & 8.47 & 0.007 \\
0.25 & -8.81 & -30027.34 & -30018.63 & 8.71 & 0.009 \\
0.33 & -9.34 & -30023.46 & -30014.38 & 9.08 & 0.065 \\
0.40 & -9.67 & -30020.89 & -30011.50 & 9.39 & 0.081 \\
\hline \hline
\end{tabular}


Based on these $\mathrm{J}^{2}$ val ues cal culated for the $\left[\mathrm{Cu}(\mathrm{IA})_{2}\right]$ complex with CAM-B3LYP* functional the best IP tuned range-separation parameter is $Y=0.20$. This is because with $\mathrm{J}^{2} \approx 0$ for a given set of $\alpha, \beta$ and $y$ (with $\alpha+\beta=1$ ) the del ocal ization error should be minimized, i.e., the energy of the neutral species as a function of the fractional electron number is very close to linear, this provides the most accurate electron density in terms of the electric field gradients. ${ }^{111}$

Assuming that the delocalization error is avoided with CAM-B3LYP* $(Y=0.20)$ a new question arises: does this variant predict with the same accuracy all the possible types of electronic transitions for the $\mathrm{Cu}(\mathrm{II})$ complex with imidazole-4-acetate?

The minimization of $\mathrm{J}^{2}$ for $\mathrm{y}=0.20$ is in particular interesting because it shows the smallest $\Delta \mathrm{Cu} \%$ for the predicted $\mathrm{d}_{\mathrm{y} z} \rightarrow \mathrm{d}_{\mathrm{x}^{2}-\mathrm{y}^{2}}$ transition, confirming to some extent, that in order to obtain reliable LF transition with LRC scheme the involved MOs should have similar character. Also, CAM-B3LYP $(Y=0.20)$ predicts three out of four $d-d$ transitions; this result is better than the experimental one where in the original spectrum only one wide absorption band is present with peak in $666 \mathrm{~nm}$ which after digital filtration splits only into two peaks at $694 \mathrm{~nm}$ and $625 \mathrm{~nm}$. Unfortunately, as mentioned before, we cannot address uniquely the true nature of such signals. Nevertheless the comparison with the original $666 \mathrm{~nm}$ is good, i.e., the excitation energy is overestimated by $0.4 \mathrm{eV}$; this value is within the expected computational accuracy at TDDFT level for open-shell metal complexes (0.3$\left.0.5 \mathrm{eV}^{131}\right)$. In addition the CAM-B3LYP* $(\mathrm{Y}=0.25)$ produces only slightly higher $\mathrm{J}^{2}$ and predicts the $\mathrm{d}_{\mathrm{yz}} \rightarrow \mathrm{d}_{\mathrm{x}^{2}-\mathrm{y}^{2}}$ transition with the same error magnitude. However the $\Delta \mathrm{Cu} \%$ is $5 \%$ larger than $\Delta \mathrm{Cu} \%$ obtained with $\gamma=0.20$.

Does the IP tuned CAM-B3LYP* (either with $\mathrm{Y}=0.20$ or $\mathrm{Y}=0.25$, as for both $\mathrm{J}^{2}$ $0.01 \mathrm{eV}^{2}$ ) show the same accuracy for other types of transitions? 
To find the answer to this question the analysis of the effect of $y$ and $\alpha$ on orbitals of different character was performed. The results will be presented and described in the next section. First the accuracy of CAM-B3LYP* with $y=0.20$ and $y=0.25$ with respect to LMCT transitions will be discussed. Selected (with $f>0.001$ ) active LCMT transitions of the $\left[\mathrm{Cu}(\mathrm{IA})_{2}\right]$ complex are presented in Table 4.14. In general a better qual itative agreement with experiment was achieved with $\gamma=0.25$. Assignment of predicted LMCT transitions is not trivial because many of these transitions are spin contaminated and/or have a highly mixed character. Moreover ligand-to-ligand transitions such as $\pi \rightarrow \pi^{*}$ (imidazole) may present a CT character and are very difficult to be identified due to the highly delocalized character of the electron acceptor. Note that for most of the LLCT transitions electron donor atoms are oxygens of the carboxylate. The maj ority of the 50 computed excited states can be described by transitions from $\beta$-spin molecular orbitals despite $\alpha$-spin MOs are also involved. Comparison of spectra produced with all the values of the rangeseparation parameter for CAM-B3LYP* functional used in this work are shown in Fig. 4.9 

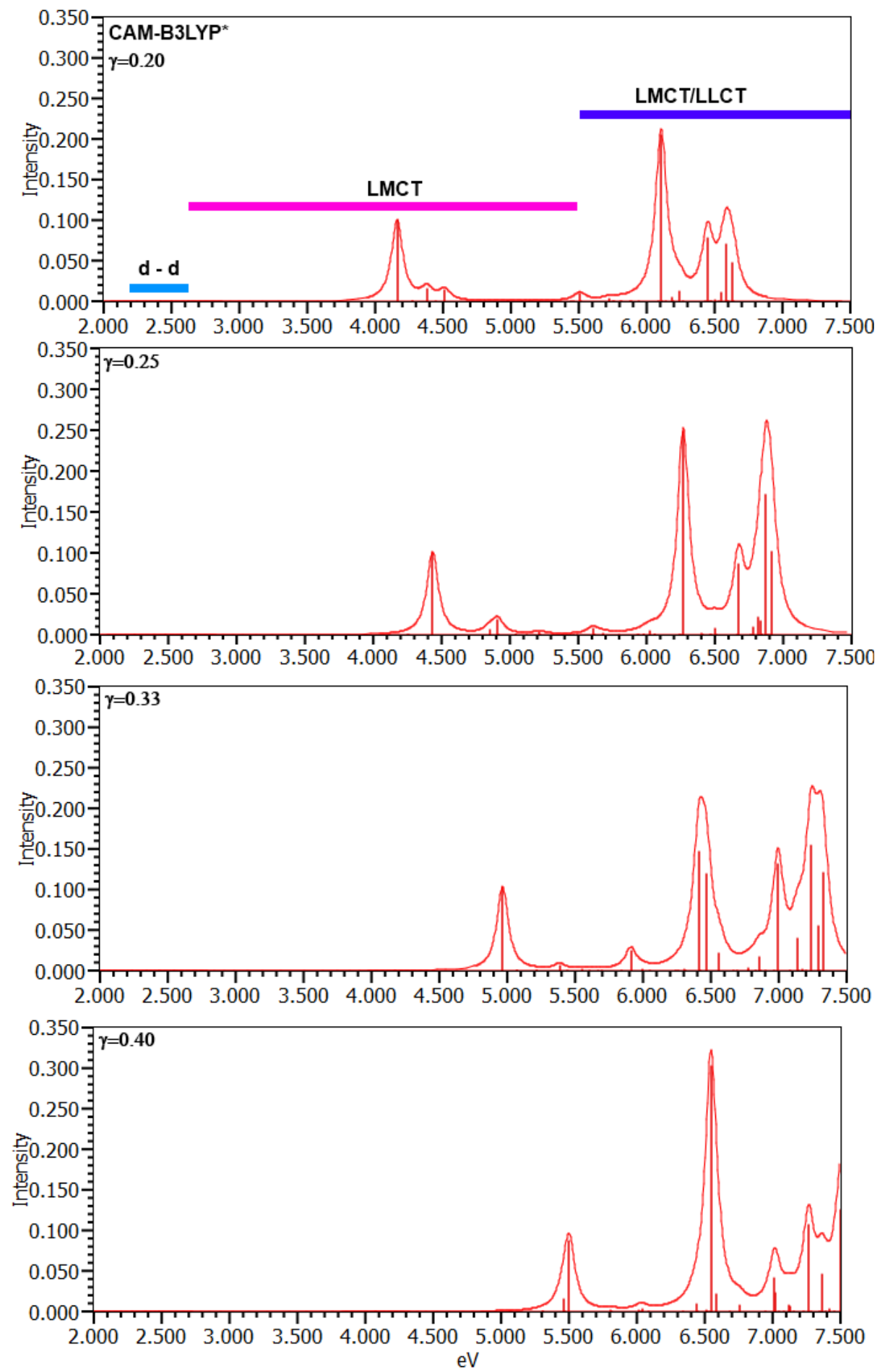

Figure 4.9: Comparison of the absorption spectra of the $\left[\mathrm{Cu}(\mathrm{IA})_{2}\right]$ complex cal culated at the CAM-B3LYP* level, employing $Y=0.20,0.25,0.33$ and 0.40 . Intensity is given in terms of the oscillator strength, and is dimensionless. 
Table 4.14: CAM-B3LYP* predicted LMCT transitions with corresponding oscillator strength (f), and $S^{2}$ for the $\left[\mathrm{Cu}(\mathrm{IA})_{2}\right]$ complex in vacuum. $\lambda$ is the wavelength, EE is the excitation energy, $\mathrm{cx}$ stands for carboxylate and im for imidazole.

\begin{tabular}{|c|c|c|c|c|c|}
\hline \multicolumn{6}{|l|}{$y=0.20$} \\
\hline Type $^{a}$ & $\lambda(\mathrm{nm})$ & $\mathrm{EE}(\mathrm{eV})$ & $\mathrm{f}$ & $S^{2}$ & $\operatorname{error}^{b}(e V)$ \\
\hline$\sigma(\mathrm{O}-\mathrm{cx}) \rightarrow \mathrm{d}_{\mathrm{Cu}^{2+}}$ & 298 & 4.17 & 0.0997 & 0.778 & 0.71 \\
\hline $\mathrm{n}(\mathrm{O}-\mathrm{cx}) \rightarrow \mathrm{d}_{\mathrm{Cu}^{2+}}$ & 283 & 4.38 & 0.0155 & 0.813 & 一 \\
\hline$\sigma / \mathrm{n}(\mathrm{O}-\mathrm{cx}) \rightarrow \mathrm{d}_{\mathrm{Cu}^{2+}}$ & 275 & 4.52 & 0.0138 & 0.786 & - \\
\hline $\mathrm{n}(\mathrm{O}-\mathrm{cx}) \rightarrow \mathrm{P}_{\mathrm{Cu}^{2+}} / \mathrm{C}(\mathrm{im} / \mathrm{cx})^{\mathrm{c}}$ & 225 & 5.51 & 0.0097 & 0.890 & - \\
\hline$\sigma / \mathrm{n}(\mathrm{O}-\mathrm{cx}) \rightarrow \mathrm{p}_{\mathrm{Cu}^{2+}} / \mathrm{C}(\mathrm{im} / \mathrm{cx})$ & 192 & 6.45 & 0.0975 & 0.975 & - \\
\hline $\mathrm{P}_{\mathrm{Cu}^{2+}} / \mathrm{n}(\mathrm{im}) \rightarrow \mathrm{d}_{\mathrm{Cu}^{2+}}$ & 187 & 6.64 & 0.0476 & 1.535 & 1.18 \\
\hline \multicolumn{6}{|l|}{$y=0.25$} \\
\hline Type $^{a}$ & $\lambda(\mathrm{nm})$ & $\mathrm{EE}(\mathrm{eV})$ & $f$ & $s^{2}$ & $\operatorname{error}^{b}(e V)$ \\
\hline$\sigma(\mathrm{O}-\mathrm{cx}) \rightarrow \mathrm{d}_{\mathrm{Cu}^{2+}}$ & 279 & 4.44 & 0.1015 & 0.778 & 0.44 \\
\hline$\sigma / \mathrm{n}(\mathrm{O}-\mathrm{cx}) \rightarrow \mathrm{d}_{\mathrm{Cu}^{2+}}$ & 255 & 4.85 & 0.0067 & 0.821 & 一 \\
\hline $\mathrm{n}(\mathrm{O}-\mathrm{cx}) \rightarrow \mathrm{d}_{\mathrm{Cu}^{2+}}$ & 252 & 4.92 & 0.0186 & 1.112 & - \\
\hline$\pi(\mathrm{C}-\mathrm{im}) \rightarrow \mathrm{d}_{\mathrm{Cu}^{2+}}$ & 218 & 5.69 & 0.0020 & 0.963 & 2.22 \\
\hline $\mathrm{n}(\mathrm{O}-\mathrm{cx}) \rightarrow \pi^{*}(\mathrm{im})^{\mathrm{c}}$ & 198 & 6.27 & 0.2487 & 0.762 & - \\
\hline $\mathrm{n}(\mathrm{O}-\mathrm{cx}) \rightarrow \mathrm{p}_{\mathrm{Cu}^{2+}} / \mathrm{C}(\mathrm{im} / \mathrm{cx})$ & 191 & 6.50 & 0.0088 & & 一 \\
\hline $\mathrm{n}(\mathrm{O}-\mathrm{cx}) \rightarrow \mathrm{p}_{\mathrm{Cu}^{2+}} / \mathrm{C}(\mathrm{im} / \mathrm{cx})^{\mathrm{d}}$ & 6.68 & 186 & 0.0866 & & - \\
\hline $\mathrm{n}(\mathrm{N}-\mathrm{im}) \rightarrow \mathrm{d}_{\mathrm{Cu}^{2+}}$ & 182 & 6.82 & 0.0222 & 1.429 & 2.23 \\
\hline $\mathrm{P}_{\mathrm{Cu}^{2+}} / \mathrm{n}(\mathrm{im}) \rightarrow \mathrm{d}_{\mathrm{Cu}^{2+}}$ & 180 & 6.88 & 0.1719 & 0.977 & 1.42 \\
\hline
\end{tabular}

a Based on $\beta$-spin population analysis of the dominating MO pair involved in a given transition unless stated differently.

${ }^{b}$ In comparison to original spectrum ${ }^{123}$ except for $\sigma(\mathrm{O}-\mathrm{cx}) \rightarrow \mathrm{d}_{\mathrm{Cu}^{2+}}$

${ }^{c}$ Based on $\alpha$-spin population analysis of the dominating MO pair.

${ }^{\mathrm{d}}$ From a different occupied molecular orbital. 


\subsubsection{More Insights About the Performance of the "Tuned" CAM- B3LYP*: Description Beyond the HOMO/LUMO Energy Levels}

Due to the fact that the best values of the range-separation parameter were chosen in relation to $19 \%$ of the short-range HF exchange it was important to check the consequence of changing the value of $\alpha$. For this purpose a set of calculations was performed where Y was set to $0.20 / 0.25$ and $\alpha$ was varied: $0.10(10 \%), 0.15(15 \%)$ and $0.25(25 \%)$. According to values of the $\mathrm{J}^{2}(\mathrm{Y}$ ), which did not change significantly (see Table 4.15) for $0.10<\alpha<0.19$ the amount of the exact exchange should not affect the spectrum. Yet minimization of $\mathrm{J}^{2}(\mathrm{Y})$ applies only to the highest occupied and unoccupied molecular orbitals, when a more general form of $\mathrm{J}^{2}$ is considered, that is when the energy of $\mathrm{HOMO}(\mathrm{N}+1)$ and EA are included. Therefore, transitions from deeper energy levels may suffer from imbalanced treatment of orbitals (for details see for example T. Körzdörfer et al. ${ }^{112}$ ).

Table 4.15: Calculated vertical IP(N) of the neutral $\left[\mathrm{Cu}(\mathrm{IA})_{2}\right]$ complex, SCF-DFT energies of neutral complex and its cation, orbital energies of $\mathrm{HOMO}(\mathrm{N})$ and $\mathrm{J}^{2}(\mathrm{Y})$ values.

\begin{tabular}{|c|c|c|c|c|c|}
\hline \multicolumn{6}{|c|}{ CAM-B3LYP* $(Y=0.20)$} \\
\hline$\alpha$ & $\varepsilon_{\text {HOMO }}(\mathrm{eV})$ & $E(N)(e V)$ & $E(N-1)(e V)$ & $\mathrm{IP}(\mathrm{eV})$ & $\mathrm{J}^{2}(\mathrm{eV})^{2}$ \\
\hline 0.10 & -8.03 & -30028.96 & -30020.84 & 8.12 & 0.008 \\
\hline 0.15 & -8.24 & -30029.92 & -30021.61 & 8.71 & 0.006 \\
\hline 0.19 & -8.39 & -30030.72 & -30022.25 & 9.08 & 0.007 \\
\hline 0.25 & -8.62 & -30031.97 & -30023.25 & 9.39 & 0.010 \\
\hline \multicolumn{6}{|c|}{ CAM-B3LYP* $(Y=0.25)$} \\
\hline$\alpha$ & $\varepsilon_{\text {HOMO }}(\mathrm{eV})$ & $\mathrm{E}(\mathrm{N})(\mathrm{eV})$ & $E(N+1)(e V)$ & $\mathrm{IP}(\mathrm{eV})$ & $\mathrm{J}^{2}(\mathrm{eV})^{2}$ \\
\hline 0.10 & -8.51 & -30025.16 & -30016.78 & 8.39 & 0.014 \\
\hline 0.15 & -8.68 & -30026.36 & -30017.79 & 8.56 & 0.013 \\
\hline 0.19 & -8.81 & -30027.34 & -30018.63 & 8.71 & 0.009 \\
\hline 0.25 & -8.99 & -30028.86 & -30019.92 & 8.94 & 0.003 \\
\hline
\end{tabular}


Indeed an analysis of orbital energies and character changes of HOMOa (noted as 75MO in Fig. 4.10), LUMOa (76MO), HOMO-11a (64MO), HOMO-10a (65MO) and HOMO9a (66MO, not shown in Fig. 4.10) orbitals upon variation of $\alpha$ and $y$ shows that HOMO and LUMO are treated differently than deeper orbitals. Figure 4.10 helps to visualize the effects of both parameters on these selected $\alpha$-spin molecular orbitals. The color coded lines represent the energetic changes; for example, the purple line represents the energetic change for the HOMO-11a. This line contai ns purple and turquoise squares, where the last indicate the different character of this orbital for $y=0.33$ and $y=0.40$. Accordingly, the yellow diamonds for HOMO-11 $\alpha$ have the same meaning, al though for this $\mathrm{MO}$ the change is less profound. This is due to the fact that their character maintains one of the d-manifold of the $\mathrm{Cu}(\mathrm{II})$ center (with some contributions form the ligand-based atomic orbital s).

The $y$-axis reports the orbital energy in electronvol ts while the $x$-axis reports either various values of the range-separation parameter $(\mathrm{Y})$ or different fractions of the exact exchange (a), Figures 4.10 and 4.11. In comparison to deeper occupied orbitals, the HOMOa do not change significantly, and a progressive increasing localization of the electron density on the imidazol e rings can be observed. Such increase is associated with slight decrease in energy. Note that this orbital may be treated as $\sigma$-type or n-type due to resonance of the carboxylate group. For the HOMO-10a, a m-type donor, the situation is different, and the change in orbital energy causes a shift between orbitals of different character. Such behavior is found when $y$ is increased from 0.25 to 0.33 . Also, the HOMO-11a which is the $\sigma$-type $\mathrm{d}_{\mathrm{z}^{2}}$ orbital of Cu changes for increasing val ues of the range parameter. This characteristic is highly visible going from 0.25 to 0.33 where this $\mathrm{MO}$ seems to swipe with another orbital composed of contributions from the $\mathrm{Cu}$ d-manifold and the ligand-based atomic orbitals. In general it is quite noticeable that $\alpha$-spin MOs are more affected by the change of the range-separation parameter than by varying the amount of the exact exchange. 

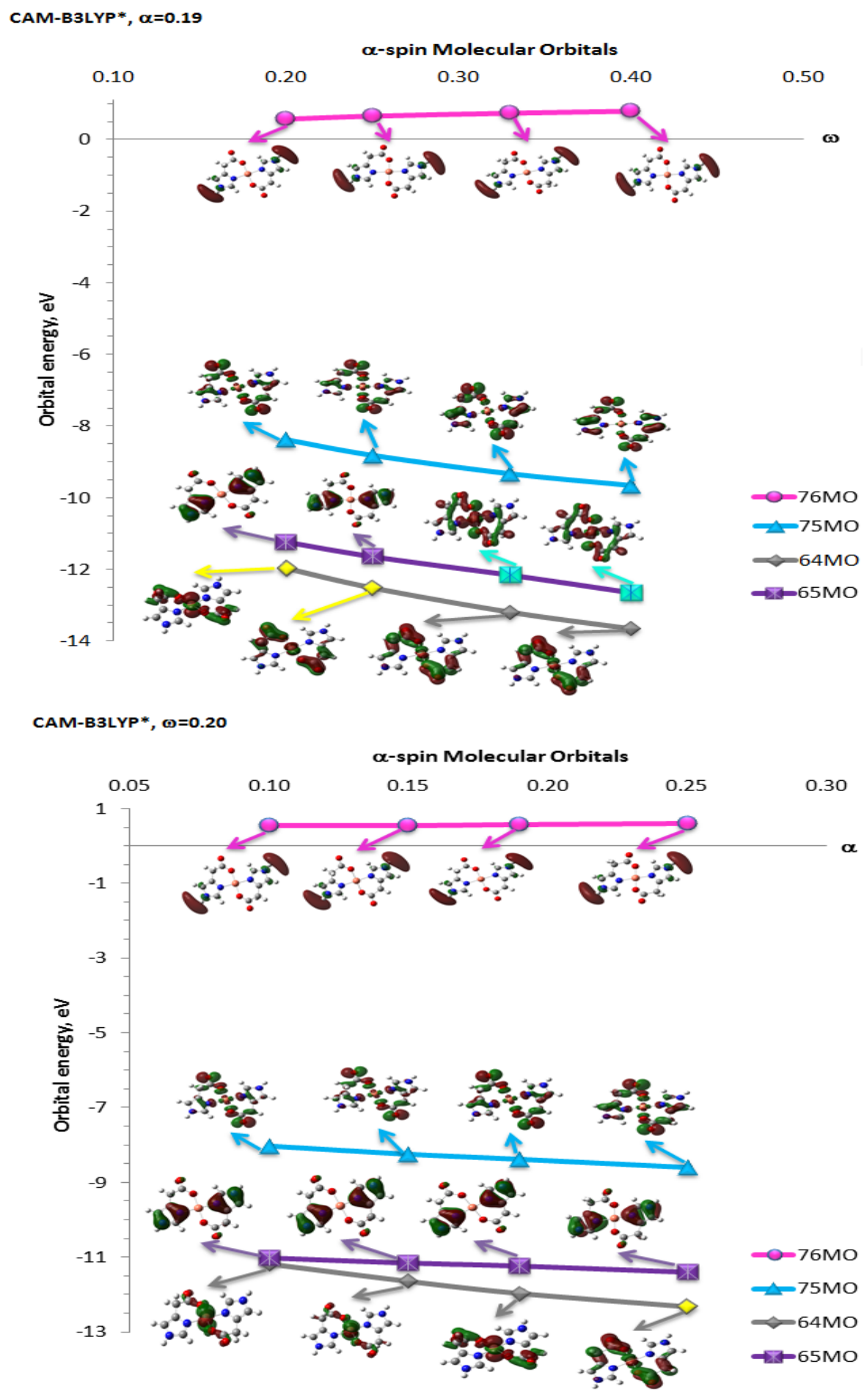

Figure 4.10: Trend for selected $\alpha$-spin Molecular Orbitals of the $\left[\mathrm{Cu}(\mathrm{IA})_{2}\right]$ complex, obtained by employing: (a) CAM-B3LYP* $(\alpha=0.19)$ with $\gamma=0.20,0.25,0.33$ and 0.40 , (b) $C A M-B 3 L Y P^{\star}(\gamma=0.20)$ with $\alpha=0.10,0.15,0.19$ and 0.25 . 


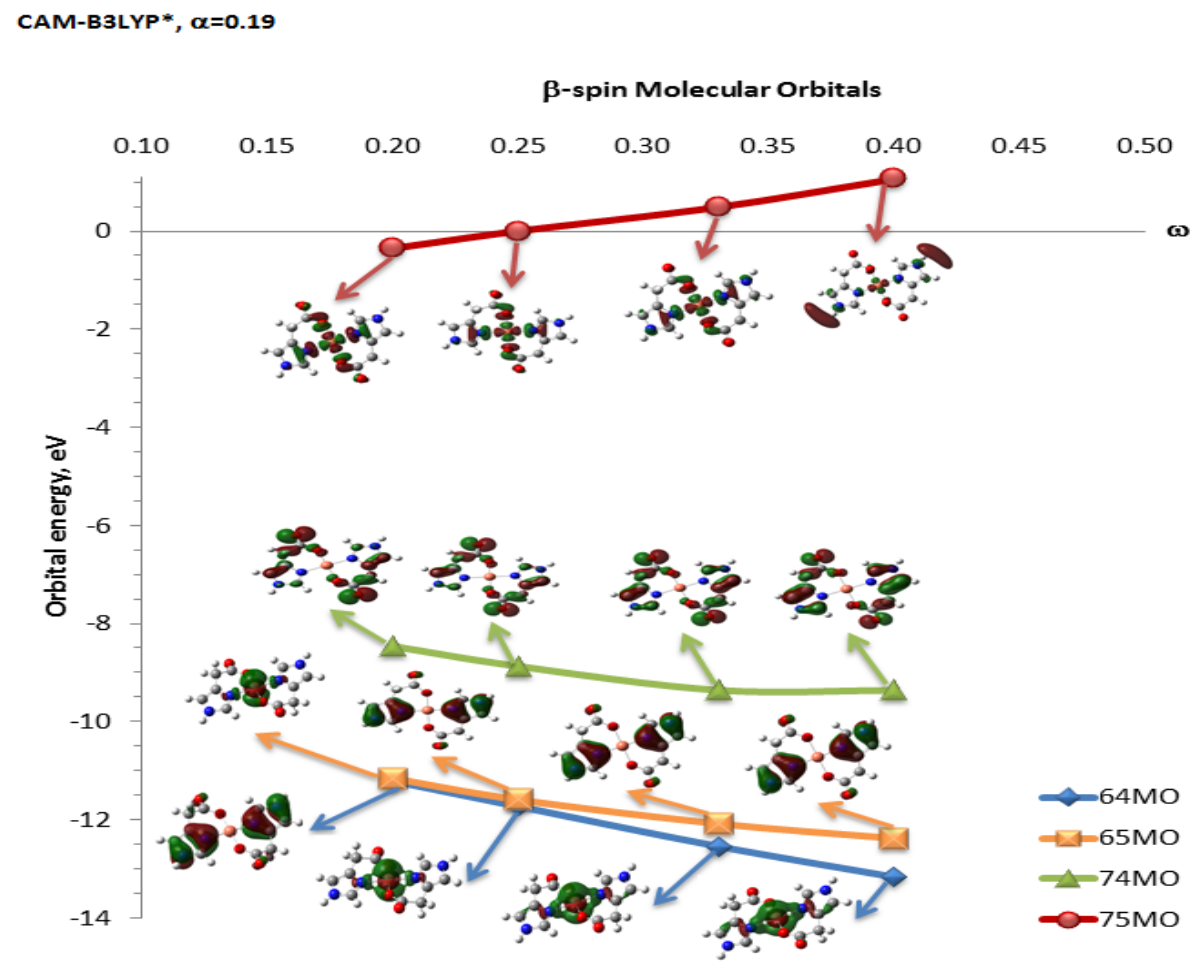

CAM-B3LYP*, $\omega=0.20$

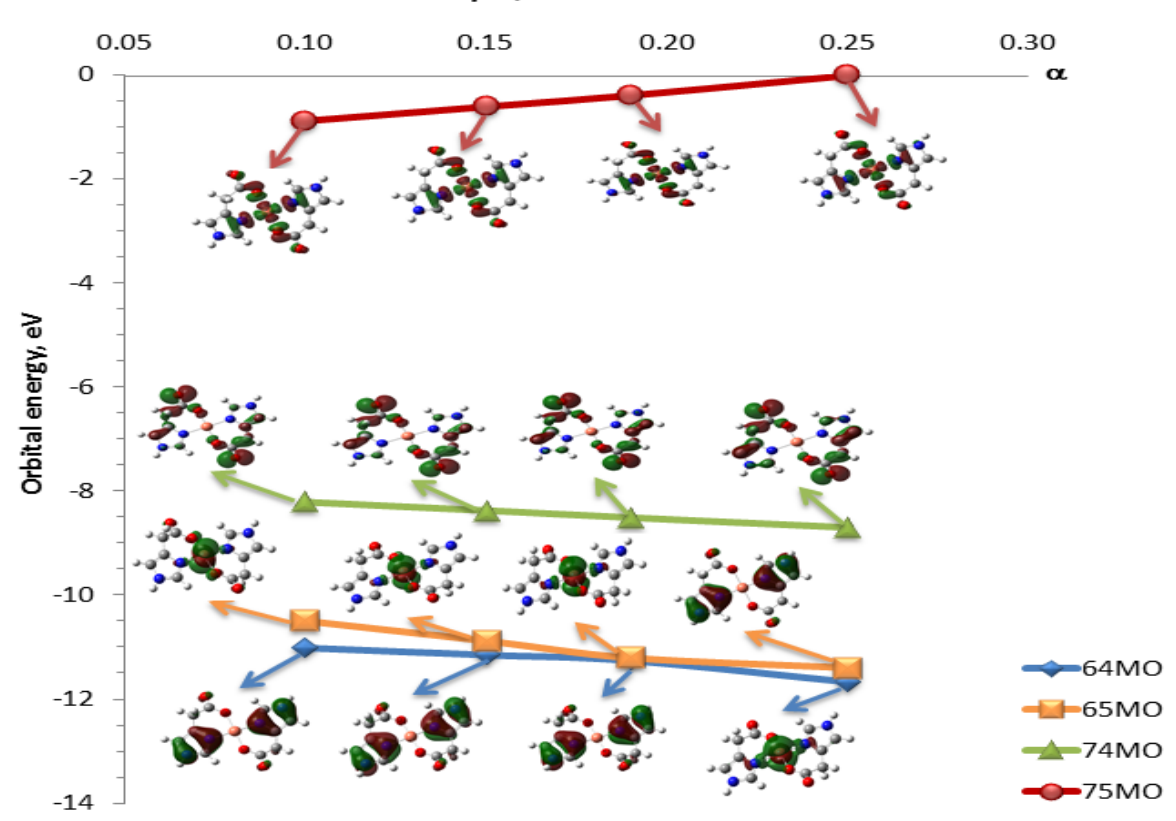

Figure 4.11: Trend for selected $\beta$-spin Molecular Orbitals of the $\left[\mathrm{Cu}(\mathrm{IA})_{2}\right]$ complex, obtained by employing: (a) CAM-B3LYP* $(\alpha=0.19)$ with $\gamma=0.20,0.25,0.33$ and 0.40 , (b) $C A M-B 3 L Y P^{\star}(\gamma=0.20)$ with $\alpha=0.10,0.15,0.19$ and 0.25 
Also, as the lowest unoccupied $\alpha$-spin MO (76MO in Fig. 4.10) does not have Cu-d character all the cal cul ated $d$ - $d$ transitions involve $\beta$-spin MOs. $\beta$-spin molecular orbital s behave very similarly to $\alpha$-spin MOs. In fact their population analysis shows that all the $\alpha$-spin orbitals overlap with $\beta$-spin orbitals (with $90 \%$ or greater correspondence) except for the SOMOa (a.k.a HOMOa) orbital that is singly occupied by the unpaired d-electron of $\mathrm{Cu}(\mathrm{II})$.

Figure 4.11 hel ps to visual ize the effects of both parameters on these selected $\beta$-spin molecular orbitals. Looking at $\beta$-spin orbital s the most significant changes occur again at deeper occupied orbital levels: HOMO-10 ( $\pi(\mathrm{N}$-imidazole) character; $64 \mathrm{MO}$ in Fig 4.11) and HOMO-9 (Cu- $\mathrm{d}_{z^{2}}, 65 \mathrm{MO}$ in Fig 4.11) turned out to be energetically flipped going from $y=0.20$ to $y=0.25$ as well as from $\alpha=0.19$ to $\alpha=0.25$. However one can al so observe a significant difference in the character of the lowest unoccupied $\beta$-spin molecular orbital (75MO) for $Y=0.40$. In fact, $L U M O \beta$ is not involved in $d-d$ transitions predicted with the CAM-B3LYP* functional when this value of range-separation parameter and $\alpha=0.19$ are used.

\subsection{3 $\left[\mathrm{Cu}(\mathrm{IAC})_{2}\right]$ : Results and Discussion}

\subsubsection{Calculations in Vacuum}

LRC-TDDFT calculations were performed for the $\left[\mathrm{Cu}(\mathrm{IAC})_{2}\right]$ complex in vacuum to establish the effect of the range-separation parameter on the position of $d-d$ transitions in UVVis spectra and on the character of involved molecular orbitals (Table 4.16 and Table4.17). Once again the investigation is focused on calculations employing various variants of the asymptotically corrected CAM-B3LYP (a.k.aCAM-B3LYP*). Note that the fraction of SR HF exchange employed for the CAM-B3LYP* with $y=0.20,0.25,0.33$ and 0.40 is $19 \%$ 
$(\alpha=0.19)$, because it has been shown that using different amounts of $\alpha$ does not necessarily improve the performance of CAM-B3LYP* (based on results for the $\left[\mathrm{Cu}(\mathrm{IA})_{2}\right]$ complex).

For $\mathrm{d}$ - $\mathrm{d}$ transitions predicted with LRC-TDDFT in vacuum a better qual itative agrement is achieved with experimental data measured in dichlorometane ( $575 \mathrm{~nm}$ or $2.16 \mathrm{eV}$ ) than in methanol (645.1 nm or $1.92 \mathrm{eV}) .{ }^{120}$ This aspect, as mentioned before (Section 4.1.2), can be explained by the fact the $\mathrm{CH}_{3} \mathrm{OH}$ interacts with $\mathrm{d}_{z^{2}}$ orbital of the $\mathrm{Cu}(\mathrm{II})$ center, which of course are not modeled by calculations in the gas-phase.

In comparison to results obtained with standard $x c$ functionals there is an evident improvement; for instance, the best estimation for $d-d$ transition was obtai ned with B3LYP $(515 \mathrm{~nm}$ or $2.1 \mathrm{eV}$ ) with an average absolute error of $0.4 \mathrm{eV}$ (i.e., the mean error obtained with respect to experimental data measured in $\mathrm{CH}_{3} \mathrm{OH}$ and $\mathrm{CH}_{2} \mathrm{Cl}_{2}$ ), which val ue is reduced by $0.2 \mathrm{eV}$ using $\omega \mathrm{B} 97 \mathrm{X}$. Although, it is important to notice that the $\mathrm{d}-\mathrm{d}$ transition predicted with B3LYP is dominated by excitation from $\beta$-spin HOMO-14 $\left(d_{x y}\right)$ to LUMO $\left(d_{x^{2}-y^{2}}\right)$, whereas $d-d$ transition predicted with wB97X involves HOMO-11 $\left(d_{y z}\right)$. Nevertheless, it seems that result from this functional is more reliable as in the square-planar symmetry the d-manifold energy levels are splitted as: $d_{x y}, d_{x z y z}, d_{z^{2}}$, and $d_{x^{2}-y^{2}}$, where these values are ordered with respect to increasing energy. Hence the transition $d_{x z y z} \rightarrow d_{x^{2}-y^{2}}$ require less energy than $d_{x y} \rightarrow d_{x^{2}-y^{2}}$. In fact, the first $d-d$ transition predicted via wB97X actually involves $d_{z^{2}} \rightarrow d_{x^{2}-y^{2}}$, but with a lower percentage of contribution to the excited state. In other words the best agreement with experimental value of the $d-d$ absorption band is with the energetically lowest excited state dominated by MOs of d-character. However, as in the case presented above, a dominating MO pair might change due to various factors (i.e., the choice of density functional itself as well as $\alpha$, $y$ parameters). 


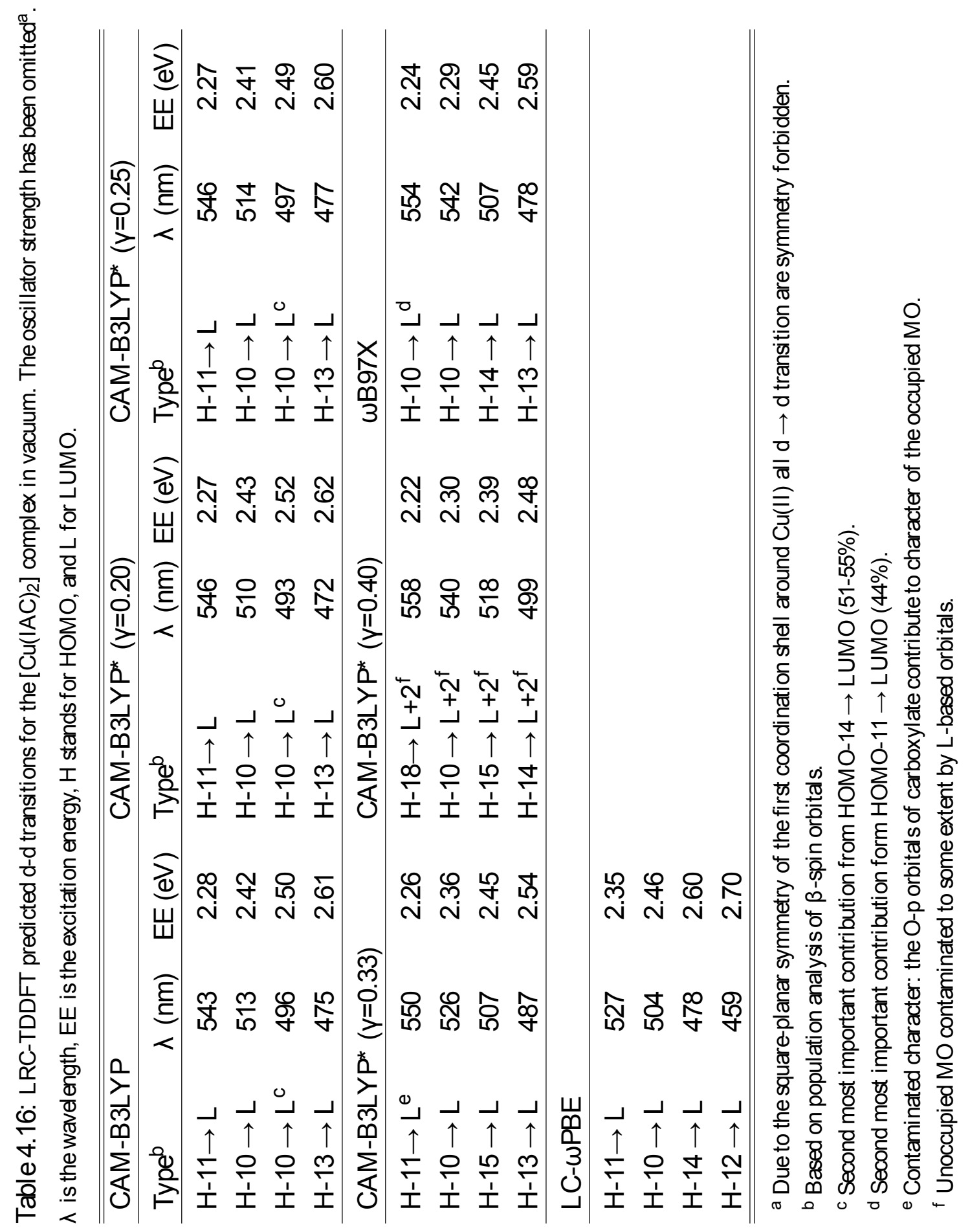


Therefore a comparison of this sort is not trivial. Moreover, although it is a common practice to examine the performance of a given theoretical methodology with respect to experimental data, such comparison may be biased if not confirmed by higher level of theory. Unfortunately, with such large systems it would be highly demanding in terms of computational effort. On the other hand even though certain assumptions made herein are based on chemical intuition and experimental data (e.g., d-splitting persists and LF transitions do not appear in the same region as LMCT transitions) others are strictly theoretical (e.g., they consider only excited states dominated by one-electron excitations, employ both longrange and asymptotic corrections, etc.). This seems to be sufficient to justify that overal LRC functionals performs better than standard xc functionals, and the good agreement with experimental data appears not to be accidental. For example, B3LYP predicts $d_{z^{2}} \rightarrow d_{x^{2}-y^{2}}$ transition at $496 \mathrm{~nm}(2.50 \mathrm{eV})$, while CAM-B3LYP* red shifts it by about $30 \mathrm{~nm}$ (i.e., to $526 \mathrm{~nm} / 2.36 \mathrm{eV}$ ); this result is in favour of the CAM-B3LYP* functional .

As shown before for the $\left[\mathrm{Cu}\left(\mathrm{IA}_{2}\right)\right]$ complex the $\mathrm{d}_{z^{2}} \rightarrow \mathrm{d}_{x^{2}-\mathrm{y}^{2}}$ transition is quite sensitive to the change of range-separation parameter. Therefore, before discussing the LMCT transitions, we shall analyze the effect of $y$ on the character and energy level of thed-manifold in its ethylated derivative. Table 4.17 summarizes the resul ts of the population anal ysis for the $\left[\mathrm{Cu}\left(\mathrm{IAC}_{2}\right)\right]$ complex while in vacuum employing variants of CAM-B3LYP* (with $\mathrm{Y}=0.20$, $0.25,0.33$ and 0.40$), \omega B 97 X$, and LC-wPBE.

Focusing on $\beta$-spin $d_{z^{2}}$ orbital (or HOMO-10) we see that for increasing value of $y$ the $\%$ contribution of $\mathrm{Cu}-\mathrm{d}$ orbital decreases and the orbital energy (OE) lowers. This behaviour is similar to that observed for $\left[\mathrm{Cu}(\mathrm{IA})_{2}\right]$. On the contrary, the LUMO $\left(\mathrm{d}_{x^{2}-y^{2}}\right.$ orbital ) gains the amount of Cu-d in the ethylated derivative of this complex, except when $y=0.40$. Energetically, the effect of range-separation on the $L U M O$ of $\left[\mathrm{Cu}(\mathrm{IAC})_{2}\right]$ is similar to that in $\left[\mathrm{Cu}(\mathrm{IA})_{2}\right]$ complex, i.e., the energy is shifted to higher val ues for increasing $\mathrm{y}$. 


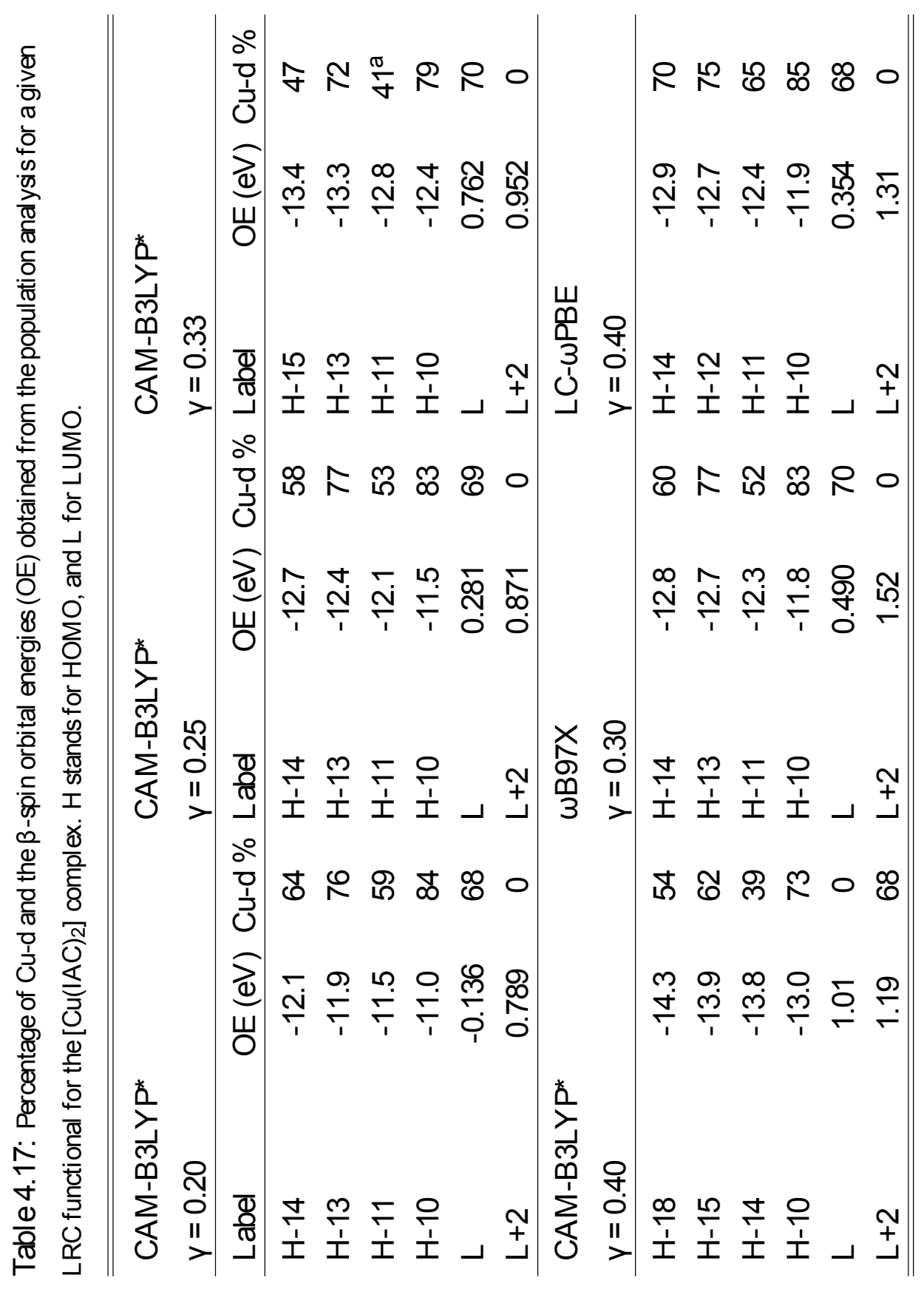


The position of the $d_{z^{2}} \rightarrow d_{x^{2}-y^{2}}$ transition in the spectra obtained with the CAM-B3LYP* variants red shifts: $493 \mathrm{~nm}(2.52 \mathrm{eV}), 514 \mathrm{~nm}(2.41 \mathrm{eV}), 526 \mathrm{~nm}(2.36 \mathrm{eV})$ and $540 \mathrm{~nm}$ $(2.30 \mathrm{eV}$ ) with $\mathrm{y}=0.20,0.25,0.33$ and 0.40 , respectively. The $\Delta \mathrm{Cu}-\mathrm{d} \%$ decreases from $16 \%$ to $5 \%(\Delta \mathrm{Cu}-\mathrm{d}=16 \%(\mathrm{y}=0.20)>\Delta \mathrm{Cu}-\mathrm{d}=14 \%(\mathrm{y}=0.25)>\Delta \mathrm{Cu}-\mathrm{d}=9 \%(\mathrm{y}=0.33)>\Delta \mathrm{Cu}-$ $d=5 \%(\gamma=0.40))$.

Again, unlike for the $\left[\mathrm{Cu}(\mathrm{IA})_{2}\right]$ complex where there was no correlation between the position of the $d_{z^{2}} \rightarrow d_{x^{2}-y^{2}}$ transition and $\Delta C u-d \%$, here the MO pair with more similar $\mathrm{Cu} \%$ is less blue shifted with respect to experimental data. ${ }^{120}$ This proves that indeed the $y$ parameter is highly system dependent ${ }^{111}$ and it affects predicted properties such as UV-Vis spectrum. Unfortunately, it is not clear if $\Delta \mathrm{Cu}-\mathrm{d} \%$ can be used as an indicator of the occurring shifts for the $d-d$ transitions. This is because this val ue appears to be unreliable, especially if the imbalanced treatment of $\sigma$-type (localized) vs. T-type (delocalized) orbitals arise (which is the case for tuned LRC hybrid functionals as shown for the $\left[\mathrm{Cu}(\mathrm{IA})_{2}\right]$ complex). Furthermore, results from cal culations preformed for $\mathrm{Cu}(\mathrm{II})$ with ethylated imidazole-4acetate complex ([Cu(IAC) $\left.)_{2}\right]$ ) employing other $\mathrm{LRC}$ functionals are not encouraging, particulary when they are compared to resultsfor $\left[\mathrm{Cu}(\mathrm{IA})_{2}\right]$. Mainly, the position of the $\mathrm{d}_{z^{2}} \rightarrow$ $d_{x^{2}-y^{2}}$ transition for both $\left[\mathrm{Cu}(I A)_{2}\right]$ and $\left[\mathrm{Cu}(\mathrm{IAC})_{2}\right]$ complexes predicted with $\omega B 97 \mathrm{X}$ is less blue shifted than the one obtained with CAM-B3LYP* (with respect to the experimental values $\left.{ }^{120,123}\right)$. However, for the $\left[\mathrm{Cu}(\mathrm{IA})_{2}\right]$ complex $\Delta \mathrm{Cu}$-d\% obtained with this functional is $13 \%$ higher than the $\Delta \mathrm{Cu}-\mathrm{d} \%$ cal culated for $\omega \mathrm{B} 97 \mathrm{X}(15 \%)$. This trend is opposite to what it was found for the $\left[\mathrm{Cu}(\mathrm{IAC})_{2}\right]$ complex, i.e., $\Delta \mathrm{Cu}-\mathrm{d}=9 \%$ and $\Delta \mathrm{Cu}-\mathrm{d}=13 \%$ for CAM-B3LYP* and $\omega B$ 97X, respectively. Furthermore, LC- $\omega P B E$ results show the position of the $d_{z^{2}} \rightarrow d_{x^{2}-y^{2}}$ transition (504 nm/2.46 eV) in the predicted spectra for the ethylated complex more blue shifted than with CAM-B3LYP* $(\gamma=0.40)(540 \mathrm{~nm} / 2.30 \mathrm{eV})$ with $\Delta \mathrm{Cu}-\mathrm{d}=5 \%$ and $\Delta \mathrm{Cu}-\mathrm{d}=17 \%$ for LC- $\omega \mathrm{PBE}$ functional. On the other hand, the position of this $d-d$ transition in the $\left[\mathrm{Cu}(\mathrm{IA})_{2}\right] \mathrm{UV}-\mathrm{Vis}$ spectraobtained with CAM-B3LYP $(\mathrm{V}=0.40)$ and LC- $\omega$ PBE is $542 \mathrm{~nm}(2.29 \mathrm{eV})$ with $\triangle \mathrm{Cu}-\mathrm{d}=20 \%$ and $505 \mathrm{~nm}(2.46 \mathrm{eV})$ with 
$\Delta \mathrm{Cu}-\mathrm{d}=18 \%$, respectively. Hence, the following conclusion can be drawn: $\Delta \mathrm{Cu}-\mathrm{d} \%$ is not the property able to reliably indicate if the position of a given $\mathrm{d}-\mathrm{d}$ transition will shift or not upon the change of $y$ and/or $\alpha$ parameter utilizing the LRC functionals.

The excitation energies with corresponding wavel engths, assignments and oscillator strengths $(f)$ of ligand-based transitions computed with CAM-B3LYP* variants are reported in Table 4.18. Note that only LMCT transitions with spin contamination less than $10 \%$, significant $f$ value (i.e., above 0.01 ) and definite character (i.e., dominating MO pair contributes at least 50\% and both donor and acceptor can be identified) are listed. Fig. 4.12 visual izes all predicated optically active excited states. Finally, results from all the other LRC functionals are reported for the LMCT transitions in Table 4.19 and Fig. 4.13.

The experimentally determined absorbance band at $260 \mathrm{~nm}(4.77 \mathrm{eV})$ in methanol and $276 \mathrm{~nm}(4.49 \mathrm{eV})$ may be assigned to a LMCT transition from $\sigma$ (O-carboxylate) to $\mathrm{d}_{\mathrm{x}^{2}-\mathrm{y}^{2}}$ orbital of $\mathrm{Cu}(\mathrm{II})$ center. ${ }^{120}$ The best agrement with values computed in vacuum with CAM-B3LYP* is for $Y=0.25$, where the transition is found at about $278 \mathrm{~nm}$ with an error of $0.02-0.3 \mathrm{eV}$. Both experimental UV-Vis spectra are measured in the presence of solvent and show only two distinct, relatively broad absorption peaks: one consistent with the value corresponding to metal-based transitions (about $2 \mathrm{eV}$ ), the other for ligand-based transitions (3.5-5.5 eV) for this type of $\mathrm{Cu}(\mathrm{II})$ complexes; ${ }^{123,125,154}$ therefore results obtained with CAM-B3LYP* $(Y=0.25)$ in vacuum agree reasonably well (the error for $d-d$ transition is in the range 0.1-0.4 eV). On the other hand, for all CAM-B3LYP* variants the maj ority of the predicted LMCT and LLCT transitions are significantly spin contaminated and/or have highly mixed character. This transitions might be dominated by double electron excitations hence cannot be correctly handled by the TDDFT which is a single electron based method (as discussed earlier). 

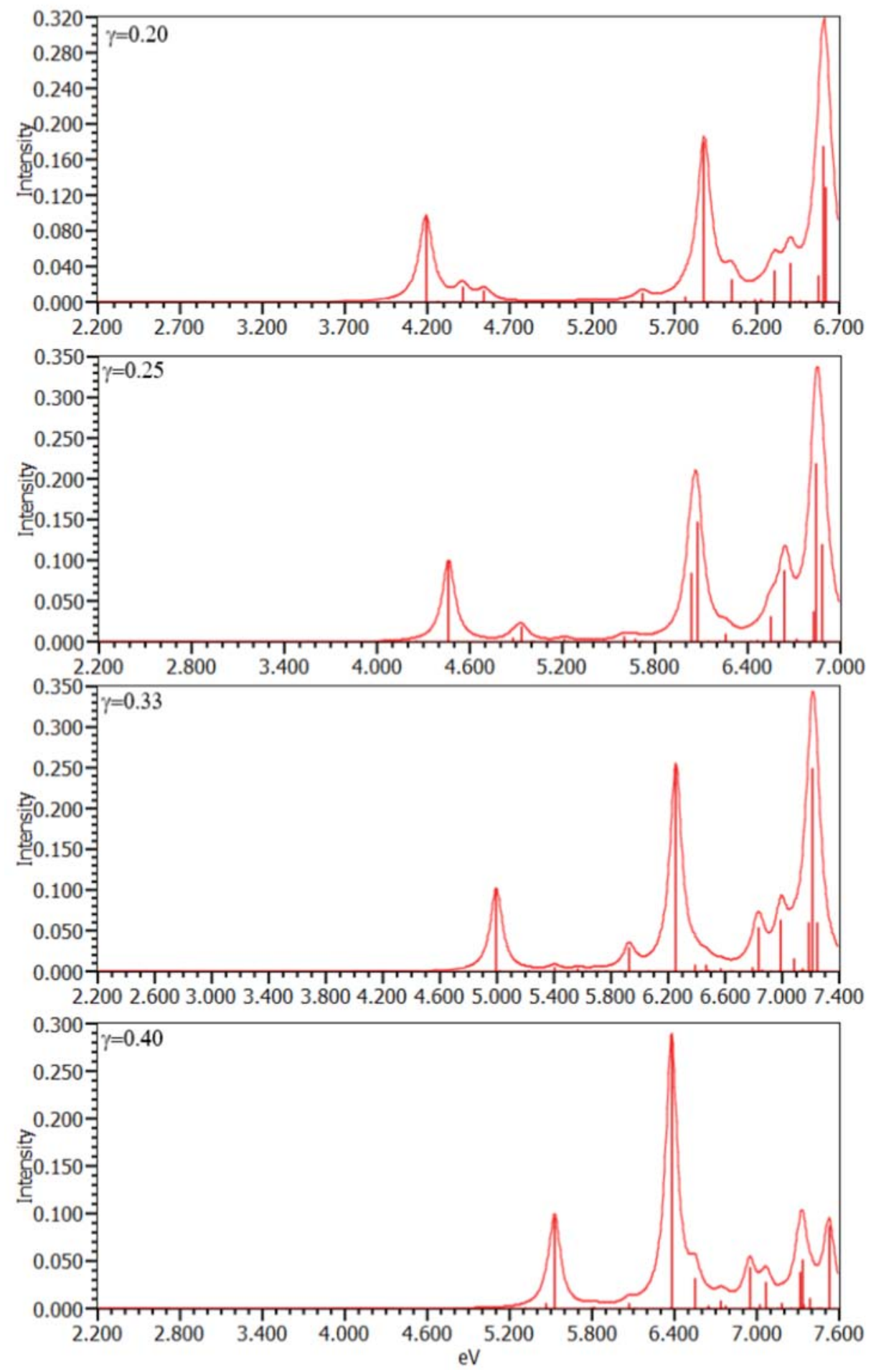

Figure 4.12: Comparison of the absorption spectra of the $\left[\mathrm{Cu}(\mathrm{IAC})_{2}\right]$ complex, cal culated with the CAMB3LYP* employing $Y=0.20,0.25,0.33$ and 0.40 
Table 4.18: CAM-B3LYP* LMCT transitions with corresponding oscillator strength (f) and $S^{2}$ for the $\left[\mathrm{Cu}(\mathrm{IAC})_{2}\right]$ complex in vacuum. $\lambda$ is the wavelength, $E E$ is the excitation energy, cx stands for carboxylate.

\begin{tabular}{|c|c|c|c|c|c|}
\hline \multicolumn{6}{|l|}{$y=0.20$} \\
\hline Type & $\lambda(\mathrm{nm})$ & EE (eV) & f & $S^{2}$ & error $^{b}(e V)$ \\
\hline$\sigma(\mathrm{O}-\mathrm{cx}) \rightarrow \mathrm{d}_{\mathrm{Cu}^{2+}}$ & 296 & 4.19 & 0.0960 & 0.790 & $0.58 / 0.30$ \\
\hline $\mathrm{n}(\mathrm{O}-\mathrm{cx}) \rightarrow \mathrm{d}_{\mathrm{Cu}^{2+}}$ & 281 & 4.38 & 0.0176 & 0.813 & - \\
\hline$\sigma / \mathrm{n}(\mathrm{O}-\mathrm{cx}) \rightarrow \mathrm{d}_{\mathrm{Cu}^{2+}}$ & 273 & 4.55 & 0.0130 & 0.787 & - \\
\hline \multicolumn{6}{|l|}{$y=0.25$} \\
\hline$\sigma(\mathrm{O}-\mathrm{cx}) \rightarrow \mathrm{d}_{\mathrm{Cu}^{2+}}$ & 278 & 4.47 & 0.0997 & 0.777 & $0.30 / 0.02$ \\
\hline \multicolumn{6}{|l|}{$y=0.33$} \\
\hline$\sigma(\mathrm{O}-\mathrm{cx}) \rightarrow \mathrm{d}_{\mathrm{Cu}^{2+}}$ & 248 & 5.00 & 0.1013 & 0.810 & $0.23 / 0.51$ \\
\hline \multicolumn{6}{|l|}{$y=0.40$} \\
\hline- & - & - & - & - & - \\
\hline $\begin{array}{l}\text { a Based on } \beta \text {-spin popul } \\
\text { involved in a given tra }\end{array}$ & $\begin{array}{l}\text { anal ysis c } \\
\text { n unless s }\end{array}$ & $\begin{array}{l}\text { the domir } \\
\text { tated differe }\end{array}$ & $\begin{array}{l}\text { nating } \mathrm{MO} \\
\text { ently. }\end{array}$ & & \\
\hline
\end{tabular}

Among the standard xc functionals employed in his work, B3LYP produced the most accurate results with respect to experimental data for the $\left[\mathrm{Cu}(\mathrm{IAC})_{2}\right]$ complex. ${ }^{120}$ However, in vacuum, the excitation energy of the first predicted d-d transition (energetically lowest) is overestimated by about $0.3-0.5 \mathrm{eV}$ (where the smal lest error is estimated by comparison with measurements in dichloromethane and the largest in methanol unless stated differently). The LMCT transition assigned as $\sigma \mathrm{O}$-carboxylate $\rightarrow \mathrm{Cu}(\mathrm{II})$ is underestimated by about $0.7-1 \mathrm{eV}$. These errors are reduced by including the LRC in this functional, i.e., for CAM-B3LYP the error for the first $d-d$ transition is $0.1-0.4 \mathrm{eV}$, whereas the LMCT transition is found with an error of 0.0-0.3 eV. The last result represents significant improvement, 
and shows that LRC is necessary to obtain a reasonable accuracy for LMCT transitions. This conclusion was not unexpected.

Table 4.19: LRC-TDDFT predicted LMCT transitions with corresponding oscillator strength (f) and $\mathrm{S}^{2}$ for the $\left[\mathrm{Cu}(\mathrm{IAC})_{2}\right]$ complex in vacuum. $\lambda$ is the wavelength, $\mathrm{EE}$ is the excitation energy, $\mathrm{cx}$ stands for carboxylate

\begin{tabular}{|c|c|c|c|c|c|}
\hline \multicolumn{6}{|l|}{ CAM-B3LYP } \\
\hline Type $^{a}$ & $\lambda(\mathrm{nm})$ & $\mathrm{EE}(\mathrm{eV})$ & $f$ & $s^{2}$ & error $^{\mathrm{b}}(\mathrm{eV})$ \\
\hline$\sigma(\mathrm{O}-\mathrm{cx}) \rightarrow \mathrm{d}_{\mathrm{Cu}^{2+}}$ & 278 & 4.46 & 0.0991 & 0.776 & $0.31 / 0.03$ \\
\hline$\sigma / \mathrm{n}(\mathrm{O}-\mathrm{cx}) \rightarrow \mathrm{d}_{\mathrm{Cu}^{2+}}$ & 259 & 4.79 & 0.0134 & 0.820 & - \\
\hline \multicolumn{6}{|l|}{$\omega B 97 X$} \\
\hline$\sigma(\mathrm{O}-\mathrm{cx}) \rightarrow \mathrm{d}_{\mathrm{Cu}^{2+}}$ & 282 & 4.55 & 0.1041 & 0.777 & $0.22 / 0.06$ \\
\hline \multicolumn{6}{|l|}{ LC- $\omega$ PBE } \\
\hline$\sigma(\mathrm{O}-\mathrm{cx}) \rightarrow \mathrm{d}_{\mathrm{Cu}^{2+}}$ & 279 & 4.40 & 0.1115 & 0.773 & $0.37 / 0.09$ \\
\hline
\end{tabular}

The asymptotically corrected CAM-B3LYP (CAM-B3LYP*) provides a slight improvement, with respect to the original version of this LRC hybrid functional, in the accuracy of the metal-based transition but it actual ly worsen the description of the ligand-based transition. Mainly, the excitation energy for the energetically lowest $d-d$ transition predicted by CAM-B3LYP* is obtained with an error of $0.1-0.3 \mathrm{eV}$ while for the LMCT ( $\sigma(\mathrm{O}-$ carboxylate) $\rightarrow$ Cu-d) transition the error is $0.2-0.5 \mathrm{eV}$ (the first error is now for comparison with $\mathrm{CH}_{3} \mathrm{OH}$ and the second with $\mathrm{CH}_{2} \mathrm{Cl}$ ). This loss of accuracy can be explained on the basis that the range-separation $(\gamma=0.33)$ as well as $\alpha=0.19$ and $\beta=0.46$ parameters (which allow to mix $B 88^{51}$ exchange and HF exchange, with $y$ dictating conversion from one to the other over the whole range) were optimized to improve excitation energies for CT tran- 

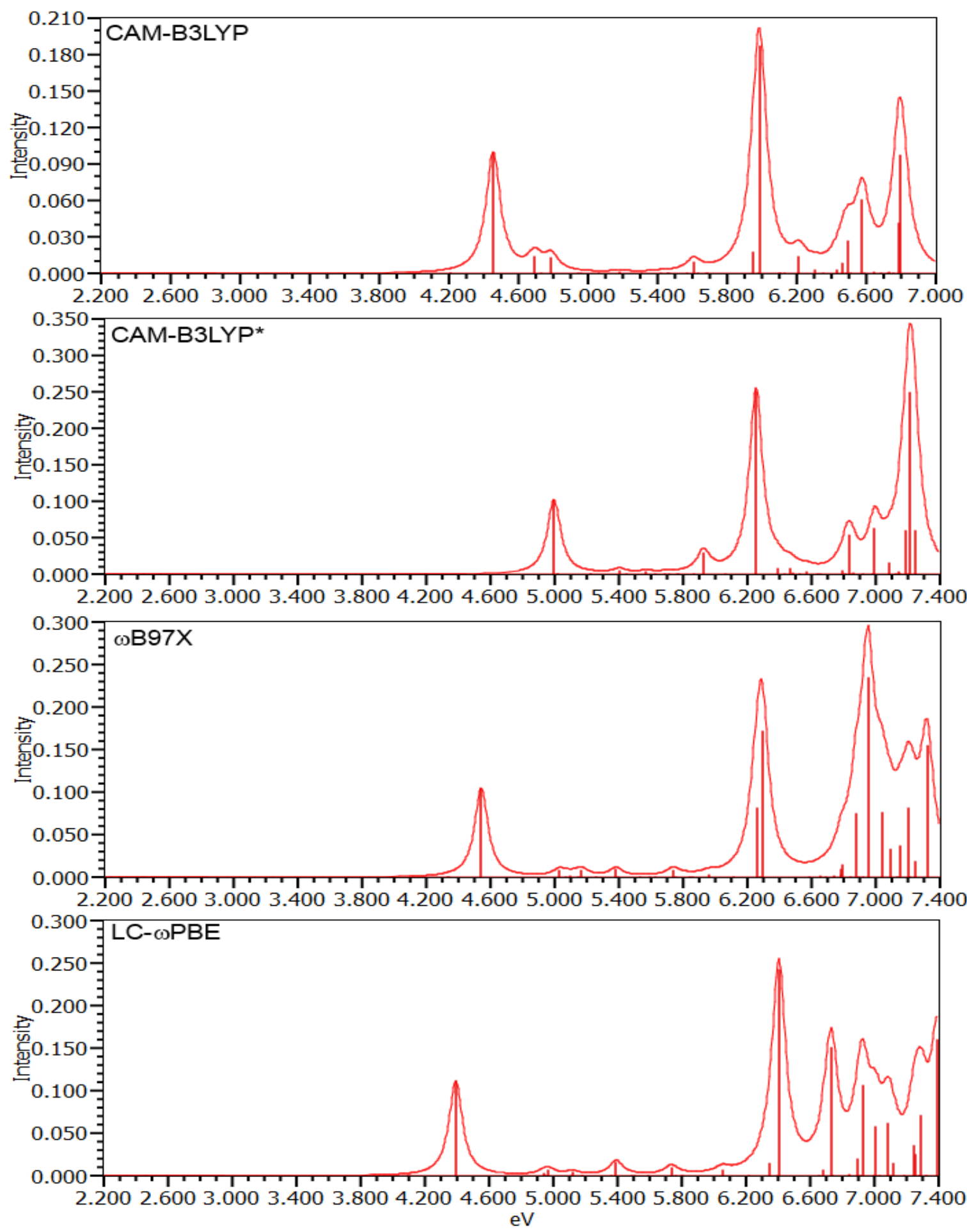

Figure 4.13: Comparison of the LRC-TDDFT absorption spectra of the $\left[\mathrm{Cu}(\mathrm{IAC})_{2}\right]$ complex in vacuum calculated with CAM-B3LYP, CAM-B3LYP* ${ }^{*} \omega B 97 X$, and LC- $\omega P B E$. Intensity is given in terms of the oscillator strength, and is dimensionless. 
sitions while keeping the accuracy of B3LYP for other energetic quantities. ${ }^{103}$ Hence, by setting $\alpha+\beta=1$ (which assures the appropriate asymptotic behaviour for the xc potential ${ }^{111}$ ) without optimizing y may lead to some undesirable effects, such as contamination of the short-range part of this potential by the extra HF exchange used to correct the longrange part. ${ }^{155}$

Considering results cal culated in vacuum with variants of CAM-B3LYP*, the best agreement with experimental spectra for the $\left[\mathrm{Cu}(\mathrm{IAC})_{2}\right]$ complex ${ }^{120}$ is achieved with $\mathrm{y}=0.25$. The excitation energy of both metal-based ligand-based transition (i.e., the first $d$ - $d$ transition and the $\sigma$ (O-carboxylate) $\rightarrow$ Cu-d transition) was found with an error of 0.1-0.3 eV. Therefore, for cal culations in vacuum this val ue of the range-separation parameter $(y=0.25)$ for CAM-B3LYP* functional is recommended. The other two LRC functionals also improved the accuracy of predicted excitation energies of these two peaks with respect to standard xc functional. In particular, a reasonably good performance for UV-Vis cal culations was given by the $\omega B 97 \mathrm{X}$ with an error of $0.1-0.3 \mathrm{eV}$ for both $\mathrm{d}-\mathrm{d}$ and LMCT transitions. LC- $\omega$ PBE performs worse for both types of transitions, increasing the error range by $0.1 \mathrm{eV}$ (i.e., $0.2-0.4 \mathrm{eV}$ ). However, even this LRC functional outperforms standard xc functional, especially for predicting position of $\sigma(\mathrm{O}$-carboxylate $) \rightarrow \mathrm{Cu}(\mathrm{II})$ transition.

\subsubsection{Calculations in Solvent}

Addition of the solvation effect of methanol and dichloromethane is expected to further improve the theoretical modeling of the UV-Vis spectra of the $\left[\mathrm{Cu}(\mathrm{IAC})_{2}\right]$ complex in LRC-TDDFT calculations. Note that we will only discuss results obtained with CAM$B 3 L Y P^{\star}(Y=0.25), C A M-B 3 L Y P^{\star}, \omega B 97 X$ and LC- $\omega P B E$ functionals. This is because CAM-B3LYP* $(Y=0.25)$ proved to provide the most accurate description of $\left[\mathrm{Cu}(\mathrm{IAC})_{2}\right]$

spectrum in vacuum and the other LRC functionals can be used to monitor effects of the 
range-separation parameter and the fraction of SR HF exchange.

Furthermore, a detailed analysis of changes in the character and the orbital energy levels of MOs upon varying $\alpha$ and $y$ in calculations with both solvents will be limited to the most important electronic transition. In particular we will refer to the $d$-d transition experimentally found around $645 \mathrm{~nm}$ (in $\mathrm{CH}_{3} \mathrm{OH}$ ) and $576 \mathrm{~nm}$ (in $\mathrm{CH}_{2} \mathrm{Cl}_{2}$ ) ${ }^{120}$ which we assigned as $d_{z^{2}} \rightarrow d_{x^{2}-y^{2}}$ based on both visual and population analysis (the first $\beta$-spin MO is labelled HOMO-10 and the second LUMO). This analysis indicate that the nature of the MOs involved in this $d-d$ transition is not affected by the solvation environment. $\beta$-spin MOs involved in the $d_{z^{2}} \rightarrow d_{x^{2}-y^{2}}$ transition in Table 4.20, which shows percentage of Cu-d and orbital energies obtained with a given LRC functional in vacuum, methanol and dichloromethane. In addition to the numerical values, Fig. 4.14 illustrates the effect of the solvated environment on $d_{z^{2}}$ and $d_{x^{2}-y^{2}}$ orbitals or rather in this case the lack of such. Results obtained with CAM-B3LYP* $(\mathrm{Y}=0.25)$ in vacuum, $\mathrm{CH}_{2} \mathrm{Cl}_{2}$, and $\mathrm{CH}_{3} \mathrm{OH}$ are showed as examples.

The HOMO-10 ( $\mathrm{d}_{z^{2}}$ orbital) is not at all affected by the solvation effect for calculations performed with all LRC functionals. The only exception is CAM-B3LYP* $(y=0.33)$, where the percentage of $\mathrm{Cu}$-d slightly decreases from $79 \%$ in vacuum to $73 \%$ in methanol. This value is only $1 \%$ higher for dichloromethane. The LUMO $\left(d_{x^{2}-y^{2}}\right)$ is even less affected as for al LRC functionals the \% of Cu-d oscillates between $68-70 \%$. This can be expected as only very small differences were found between structures obtained with the solvation environment of methanol and dichloromethane, and corresponding parameters in vacuum. On the other hand, significant shifts in the position of this transition upon change from vacuum to either solvent are observed (Table 4.21). For example, for cal culations in vacuum using CAM-B3LYP $(y=0.25)$ the $d_{z^{2}} \rightarrow d_{x^{2}-y^{2}}$ transition is found at $514 \mathrm{~nm}(2.41 \mathrm{eV})$, in $\mathrm{CH}_{2} \mathrm{Cl}_{2}$ it red shifts to $547 \mathrm{~nm}(2.27 \mathrm{eV})$, but changing from dichloromethane to methanol does not cause any significant shifts (i.e., in $\mathrm{CH}_{3} \mathrm{OH}$ it is found at $548 \mathrm{~nm} / 2.26 \mathrm{eV}$ ). 
Table 4.20: Procentage of Cu-d and $\beta$-spin orbital energies (OE) obtained from population analysis with given $\mathrm{LRC}$ functional for the $\left[\mathrm{Cu}(\mathrm{IAC})_{2}\right]$ complex in vacuum, $\mathrm{CH}_{3} \mathrm{OH}$ and $\mathrm{CH}_{2} \mathrm{Cl}_{2}$. $\mathrm{H}$ stands for $\mathrm{HOMO}$ and $\mathrm{L}$ for LUMO

\begin{tabular}{|c|c|c|c|c|c|}
\hline \multicolumn{5}{|c|}{ VACUUM } & \\
\hline \multicolumn{2}{|c|}{ CAM-B3LYP* $(Y=0.25)$} & \multicolumn{4}{|c|}{ CAM-B3LYP* $(Y=0.33)$} \\
\hline Label & $\mathrm{OE}(\mathrm{eV})$ & Cu-d \% & Label & $\mathrm{OE}(\mathrm{eV})$ & Cu-d \% \\
\hline $\mathrm{H}-10$ & -11.0 & 83 & $\mathrm{H}-10$ & -12.4 & 79 \\
\hline $\mathrm{L}$ & 0.224 & 69 & $\mathrm{~L}$ & 0.756 & 70 \\
\hline \multicolumn{2}{|l|}{$\omega B 97 X$} & & \multicolumn{3}{|c|}{ LC- $\omega$ PBE } \\
\hline $\mathrm{H}-10$ & -11.8 & 83 & $\mathrm{H}-10$ & -11.9 & 85 \\
\hline \multirow[t]{2}{*}{$\mathrm{L}$} & 0.482 & 70 & $\mathrm{~L}$ & 0.364 & 68 \\
\hline & & \multicolumn{4}{|l|}{$\mathrm{CH}_{3} \mathrm{OH}$} \\
\hline \multicolumn{2}{|c|}{ CAM-B3LYP* $(Y=0.25)$} & & \multicolumn{3}{|c|}{ CAM-B3LYP* $(Y=0.33)$} \\
\hline Label & $\mathrm{OE}(\mathrm{eV})$ & Cu-d \% & Label & $\mathrm{OE}(\mathrm{eV})$ & Cu-d \% \\
\hline $\mathrm{H}-10$ & -11.9 & 80 & $\mathrm{H}-10$ & -12.7 & 73 \\
\hline L & -0.230 & 69 & $\mathrm{~L}$ & 0.307 & 70 \\
\hline \multicolumn{2}{|l|}{$\omega B 97 X$} & & \multicolumn{3}{|c|}{ LC- $\omega P B E$} \\
\hline $\mathrm{H}-10$ & -12.1 & 80 & $\mathrm{H}-10$ & -12.2 & 82 \\
\hline \multirow{2}{*}{\multicolumn{2}{|c|}{0.026}} & 70 & $\mathrm{~L}$ & -0.113 & 69 \\
\hline & & $\mathrm{CH}_{2} \mathrm{Cl}_{2}$ & & & \\
\hline \multicolumn{3}{|c|}{ CAM-B3LYP ${ }^{*} \gamma=0.25$} & \multicolumn{3}{|c|}{ CAM-B3LYP* $(Y=0.33)$} \\
\hline Label & $\mathrm{OE}(\mathrm{eV})$ & Cu-d \% & Label & $\mathrm{OE}(\mathrm{eV})$ & Cu-d \% \\
\hline $\mathrm{H}-10$ & -11.9 & 80 & $\mathrm{H}-10$ & -12.7 & 74 \\
\hline L & -0.202 & 69 & L & 0.756 & 70 \\
\hline \multicolumn{3}{|l|}{$\omega B 97 X$} & \multicolumn{3}{|c|}{ LC- $\omega$ PBE } \\
\hline $\mathrm{H}-10$ & -12.1 & 80 & $\mathrm{H}-10$ & -12.2 & 83 \\
\hline $\mathrm{L}$ & 0.051 & 70 & $\mathrm{~L}$ & -0.0846 & 69 \\
\hline
\end{tabular}




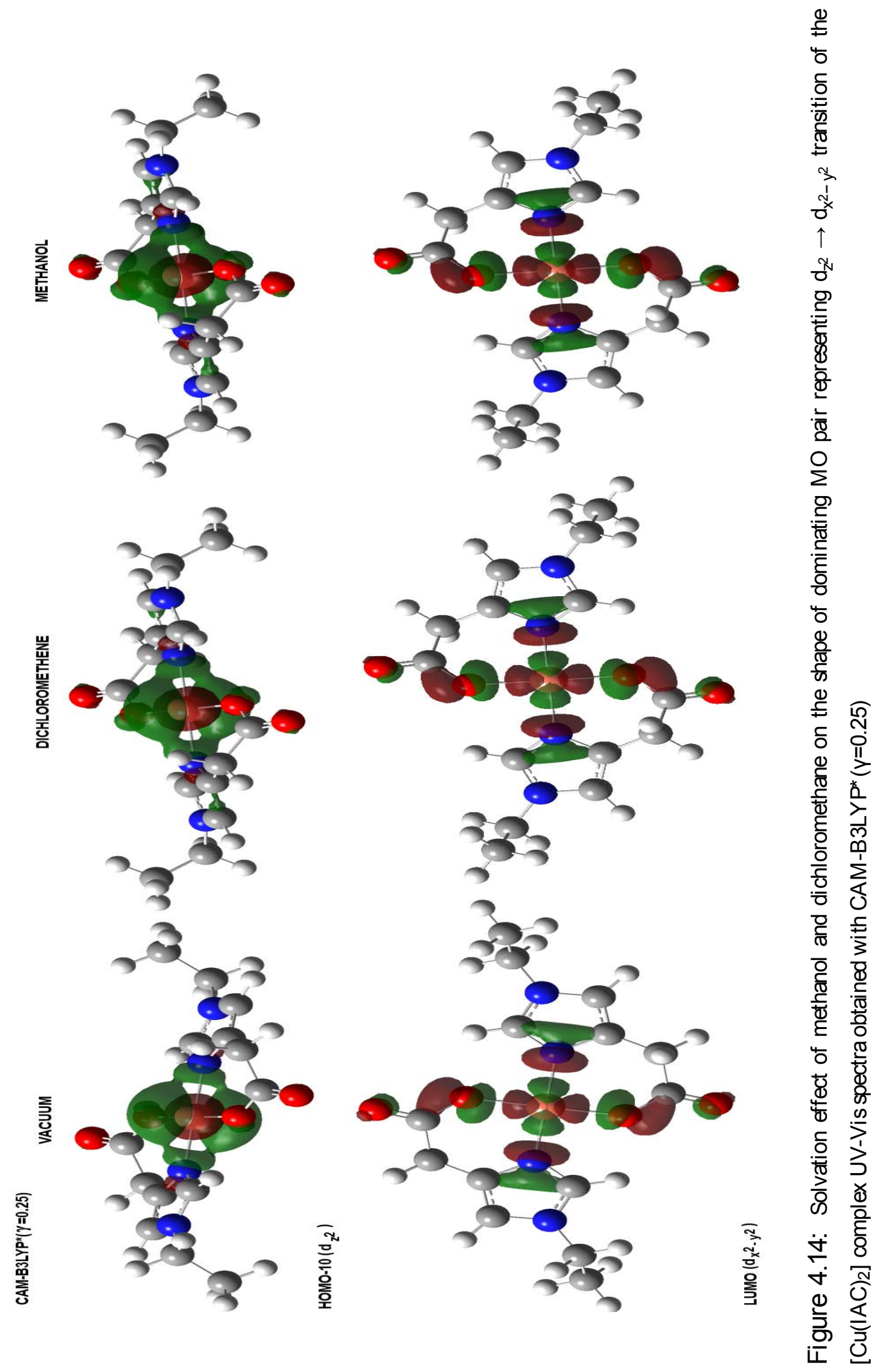


One possible explanation for this behaviour is that the experimental ly observed absorption band is formed by a doubly excited state. The rationalization behind this statement the following: two excited states involving the same dominating MO pair were found. One of them showed a weight factor slightly smaller than that of the other one. For instance, from cal culations with CAM-B3LYP* $(y=0.25)$ including the solvation effect of methanol we found that the first excited state (2.26 eV) have first dominating HOMO-10/LUMO pair (weight factor of 0.61 or $61 \%$ ) while the second most important HOMO-13/LUMO pair accounts for $41 \%$. The second excited state was predicted at $2.31 \mathrm{eV}$, for which HOMO10/LUMO pair contributes 57\% and HOMO-13/LUMO 49\%. Interestingly, the LC- $\omega P B E$ results are different. In fact, LC- $\omega P B E$ is the only LRC functional which does not produce the "twin" excited states in either environment. This aspect and the fact that the value of $S^{2}$ for each of these excited states is about 0.75 , suggest that these states are pure doublet-doublet transitions. Therefore, the above reasonings is unlikely to be the correct explanation for the actual cause of shifts occurring in the $d_{z^{2}} \rightarrow d_{x^{2}-y^{2}}$ transition. A more possible interpretation of the discrepancy between the lack of effect due to the solvation environment on the HOMO-10/LUMO pair and the predicted significant shifts lies again in the imbalanced treatment of orbitals of a different nature. Mainly, each of the "twin" excited states has a large contribution from the HOMO-10/LUMO pair, both localized ( $\sigma$ type) MOs and the HOMO-13/LUMO pair, where HOMO-13 is either one of $d_{x z, y z}$ or $d_{x y}$ which are delocalized ( $\pi$-type) MOs. Thus, the excitation energies of the excited states where dominating MO pairs composed of these two types of orbitals are mixed, might be affected by the unbal anced LR correction of $\sigma$ - vs. $\pi$-type. On the other hand similar shifts in the position of the d-d transitions from vacuum to either solvent is observed also for cal culations with standard $x c$ functionals. Hence at this point we claim that this effect is due to the change of environment even though its effect is not explicitly observable from the orbitals.

Table 4.21 presents the $d$-d transitionsfor the $\left[\mathrm{Cu}(\mathrm{IAC})_{2}\right]$ complex in methanol and dichloro methane; the LMCT transitions are reported in Table 4.22. 
Table 4.21: LRC-TDDFT predicted d-d transitions for the $\left[\mathrm{Cu}(\mathrm{IAC})_{2}\right]$ complex in methanol and dichlorometane. The oscillator strength has been omitted. ${ }^{a} \lambda$ is the wavelength, $E E$ is the excitation energy, $\mathrm{H}$ stands for HOMO, and L for LUMO.

\begin{tabular}{|c|c|c|c|c|c|}
\hline \multirow{2}{*}{\multicolumn{2}{|c|}{ CAM-B3LYP* $(\gamma=0.25)$}} & \multicolumn{4}{|c|}{$E x p=645 \mathrm{~nm}(1.92 \mathrm{eV})^{120}$} \\
\hline & & \multicolumn{4}{|c|}{ CAM-B3LYP* $(\gamma=0.33)$} \\
\hline Type ${ }^{b}$ & $\lambda(\mathrm{nm})$ & $\mathrm{EE}(\mathrm{eV})$ & Type $^{b}$ & $\lambda(\mathrm{nm})$ & $\mathrm{EE}(\mathrm{eV})$ \\
\hline $\mathrm{H}-10 \rightarrow \mathrm{L}$ & 548 & 2.26 & $\mathrm{H}-10 \rightarrow \mathrm{L}$ & 562 & 2.21 \\
\hline$H-10 \rightarrow L^{f}$ & 536 & 2.31 & $\mathrm{H}-14 \rightarrow \mathrm{L}$ & 547 & 2.27 \\
\hline $\mathrm{H}-15 \rightarrow \mathrm{L}$ & 522 & 2.37 & $\mathrm{H}-15 \rightarrow \mathrm{L}$ & 533 & 2.33 \\
\hline $\mathrm{H}-17 \rightarrow \mathrm{L}$ & 492 & 2.52 & $\mathrm{H}-15 \rightarrow \mathrm{L}^{\mathrm{C}}$ & 503 & 2.47 \\
\hline$\omega B 97 X$ & & & LC- $\omega$ PBE & & \\
\hline $\mathrm{H}-10 \rightarrow \mathrm{L}$ & 585 & 2.12 & $\mathrm{H}-10 \rightarrow \mathrm{L}$ & 538 & 2.30 \\
\hline $\mathrm{H}-13 \rightarrow \mathrm{L}$ & 537 & 2.31 & $\mathrm{H}-12 \rightarrow \mathrm{L}$ & 519 & 2.39 \\
\hline $\mathrm{H}-15 \rightarrow \mathrm{L}^{\mathrm{d}}$ & 525 & 2.36 & $\mathrm{H}-16 \rightarrow \mathrm{L}$ & 500 & 2.48 \\
\hline \multirow[t]{2}{*}{$\mathrm{H}-15 \rightarrow \mathrm{L}$} & 495 & 2.50 & $\mathrm{H}-13 \rightarrow \mathrm{L}$ & 472 & 2.63 \\
\hline & & $\mathrm{CH}_{2} \mathrm{Cl}_{2}$ & \multirow{2}{*}{\multicolumn{3}{|c|}{ CAM-B3LYP* $(\gamma=0.33)$}} \\
\hline \multicolumn{3}{|c|}{ CAM-B3LYP* $(\gamma=0.25)$} & & & \\
\hline Type & $\lambda(\mathrm{nm})$ & $\mathrm{EE}(\mathrm{eV})$ & Type $^{b}$ & $\lambda(\mathrm{nm})$ & $\mathrm{EE}(\mathrm{eV})$ \\
\hline $\mathrm{H}-10 \rightarrow \mathrm{L}$ & 547 & 2.27 & $\mathrm{H}-10 \rightarrow \mathrm{L}$ & 559 & 2.22 \\
\hline $\mathrm{H}-10 \rightarrow \mathrm{L}^{\mathrm{e}}$ & 535 & 2.32 & $\mathrm{H}-13 \rightarrow \mathrm{L}$ & 546 & 2.27 \\
\hline $\mathrm{H}-14 \rightarrow \mathrm{L}$ & 520 & 2.38 & $\mathrm{H}-15 \rightarrow \mathrm{L}^{\mathrm{c}}$ & 551 & 2.34 \\
\hline $\mathrm{H}-17 \rightarrow \mathrm{L}$ & 490 & 2.53 & $\mathrm{H}-15 \rightarrow \mathrm{L}$ & 501 & 2.47 \\
\hline$\omega B 97 X$ & & & LC- $\omega$ PBE & & \\
\hline $\mathrm{H}-10 \rightarrow \mathrm{L}$ & 581 & 2.13 & $\mathrm{H}-10 \rightarrow \mathrm{L}$ & 535 & 2.32 \\
\hline $\mathrm{H}-12 \rightarrow \mathrm{L}$ & 537 & 2.31 & $\mathrm{H}-11 \rightarrow \mathrm{L}$ & 518 & 2.39 \\
\hline $\mathrm{H}-17 \rightarrow \mathrm{L}$ & 523 & 2.37 & $\mathrm{H}-14 \rightarrow \mathrm{L}$ & 498 & 2.49 \\
\hline $\mathrm{H}-13 \rightarrow \mathrm{L}$ & 493 & 2.51 & $\mathrm{H}-13 \rightarrow \mathrm{L}$ & 470 & 2.64 \\
\hline
\end{tabular}

${ }^{a}$ Due to the square-planar symmetry of the first coordination shell around $\mathrm{Cu}(\mathrm{II})$ all $\mathrm{d} \rightarrow \mathrm{d}$ transition are symmetry forbidden.

${ }^{b}$ Based on population analysis of $\beta$-spin orbitals.

c Second most important contribution from HOMO-18 $\rightarrow$ LUMO

d Second most important contribution form HOMO-17 $\rightarrow$ LUMO

e Second most important contribution form HOMO-14 $\rightarrow$ LUMO

f Second most important contribution form HOMO-13 $\rightarrow$ LUMO 


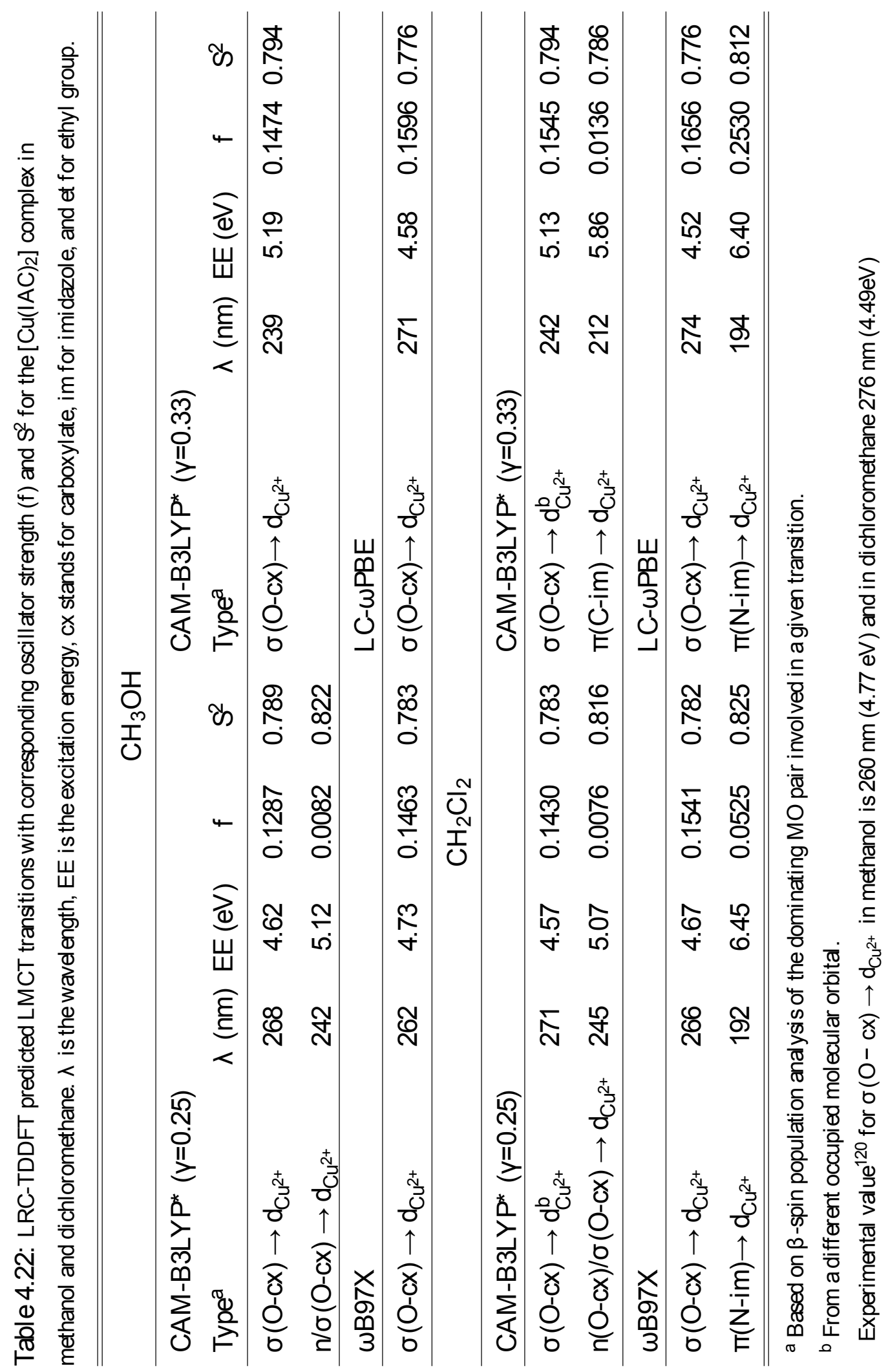


As discussed earlier for both standard and LRC xc functionals we observed a lowering of the excitation energy for $d$-d transitions upon involving either solvent in the calculations. However, when changing environment from methanol to dichloromethane there is either too small solvatochromic shift compare to experimental data ${ }^{120}$ or there is no effect at all. For example, with CAM-B3LYP* $(\gamma=0.33)$ the predicted position of the $d-d$ transition is red shifted from $526 \mathrm{~nm}(2.36 \mathrm{eV})$ in vacuum to $559 \mathrm{~nm}(2.22 \mathrm{eV})$ in dichloromethane and $562 \mathrm{~nm}(2.21 \mathrm{eV})$ in methanol. Unfortunately, once again (i.e., as it was for cal culations using standard $x c$ functionals) the expected blue shift for results including the solvation effect of $\mathrm{CH}_{2} \mathrm{Cl}_{2}$ with respect to $\mathrm{CH}_{3} \mathrm{OH}$ is correct but far too small. In fact, experimentally measured position of the absorption band assigned as $d-d$ transition in methanol is found at $645 \mathrm{~nm}(1.92 \mathrm{eV})$ and $575 \mathrm{~nm}(2.16 \mathrm{eV})$ in dichloromethane, giving a blue shift of about $70 \mathrm{~nm}(0.2 \mathrm{eV}) .{ }^{120}$ Since it was not clear if the y parameter or for that matter al so $\alpha$ influenced the accuracy of the predicted solvatochromic shifts, further analysis of this solvation effect was carried out with other LRC functionals. First, it should be pointed out that $C A M-B 3 L Y P^{\star}(\gamma=0.25)$ gives similar results as its variant with $y=0.33$, except that the $d_{z^{2}} \rightarrow d_{x^{2}-y^{2}}$ transition is blue shifted by about $12 \mathrm{~nm}$ in vacuum, $12 \mathrm{~nm}$ in $\mathrm{CH}_{2} \mathrm{Cl}_{2}$, and $14 \mathrm{~nm}$ in $\mathrm{CH}_{3} \mathrm{OH}$ with respect to val ues obtained using larger $\mathrm{y}$. Furthermore, wB97X and $L C-\omega P B E$ perform similarly to CAM-B3LYP* $(y=0.25)$, which gives only slightly worst results than CAM-B3LYP*. This suggests that if $Y$ has any influence on the solvatochromic shifts its effect is very small and can be neglected. Such conclusion seems to be reasonable but it does not fully address the complexity of the problem. For instance, the consequences of using various amounts of the exact exchange $(\alpha)$ were not discussed yet. Another issue that may be the cause of poor performance of the LRC functionals in terms of predicting solvatochromic shifts going from methanol to dichloromethane (or vice versa) is the choice of the IEF-PCM approach itself that may not be appropriate to mimic this effect. In fact this conclusion was al ready stated for standard xc functionals and the results with LRC functionals seems to confirm it. Hence this model for the $\left[\mathrm{Cu}(\mathrm{IAC})_{2}\right]$ complex should be applied with some caution, as it cannot properly account for the solvatochromic shifts 
observed experimentally. Yet, it is important to stress again that in comparison to results in vacuum the involvement of solvent via the IEF-PCM approach allows to improve the agreement between theory and experiment.

Fig 4.15 shows predicted transitions by CAM-B3LYP $(\gamma=0.25), \omega B 97 X$, and LC- $\omega P B E$ functionals in both solvents. CAM-B3LYP* is not reported because the comparison between UV-Vis spectra predicted by this variant and CAM-B3LYP* $(Y=0.25)$ will be shown separately in Fig 4.16 together with the spectrum in vacuum. This choice is adopted to clearly illustrate the solvation effect with respect to the change of the range-separation parameter.

Oliveira and coworkers ${ }^{156}$ studied the solvatochromic shift of the pyridinium- $\mathrm{N}$-phenoxide betaine dye using the long-range corrected functionals. These authors reports that CAMB3LYP gives smaller blue solvation shift than LC- $\omega P B E$ for the $\pi-\pi^{*}$ transition. Since LRC functional has larger $y$ value than CAM-B3LYP ( $y=0.40$ vs. $\gamma=0.33)$, it can be argued that a lager value of the range-separation may cause larger shift. Although, results for our systems show al most the same shift for the $d_{z^{2}} \rightarrow d_{x^{2}-y^{2}}$ transition cal culated via $C A M-B 3 L Y P^{*}(y=0.25)$ and CAM-B3LYP* with the change of the environment from vacuum to methanol, i.e., from $514 \mathrm{~nm} / 2.41 \mathrm{eV}$ to $548 \mathrm{~nm} / 2.26 \mathrm{eV}$ for $\mathrm{\gamma}=0.25$, and from $526 \mathrm{~nm} / 2.36 \mathrm{eV}$ to $562 \mathrm{~nm} / 2.21 \mathrm{eV}$ for CAM-B3LYP*. Note that LC- $\omega P B E$ and CAMB3LYP besides representing two different LRC functionals al so have a different amounts of SR HF exchange, whereas both the employed variants of CAM-B3LYP* have the same amount of SR HF. 


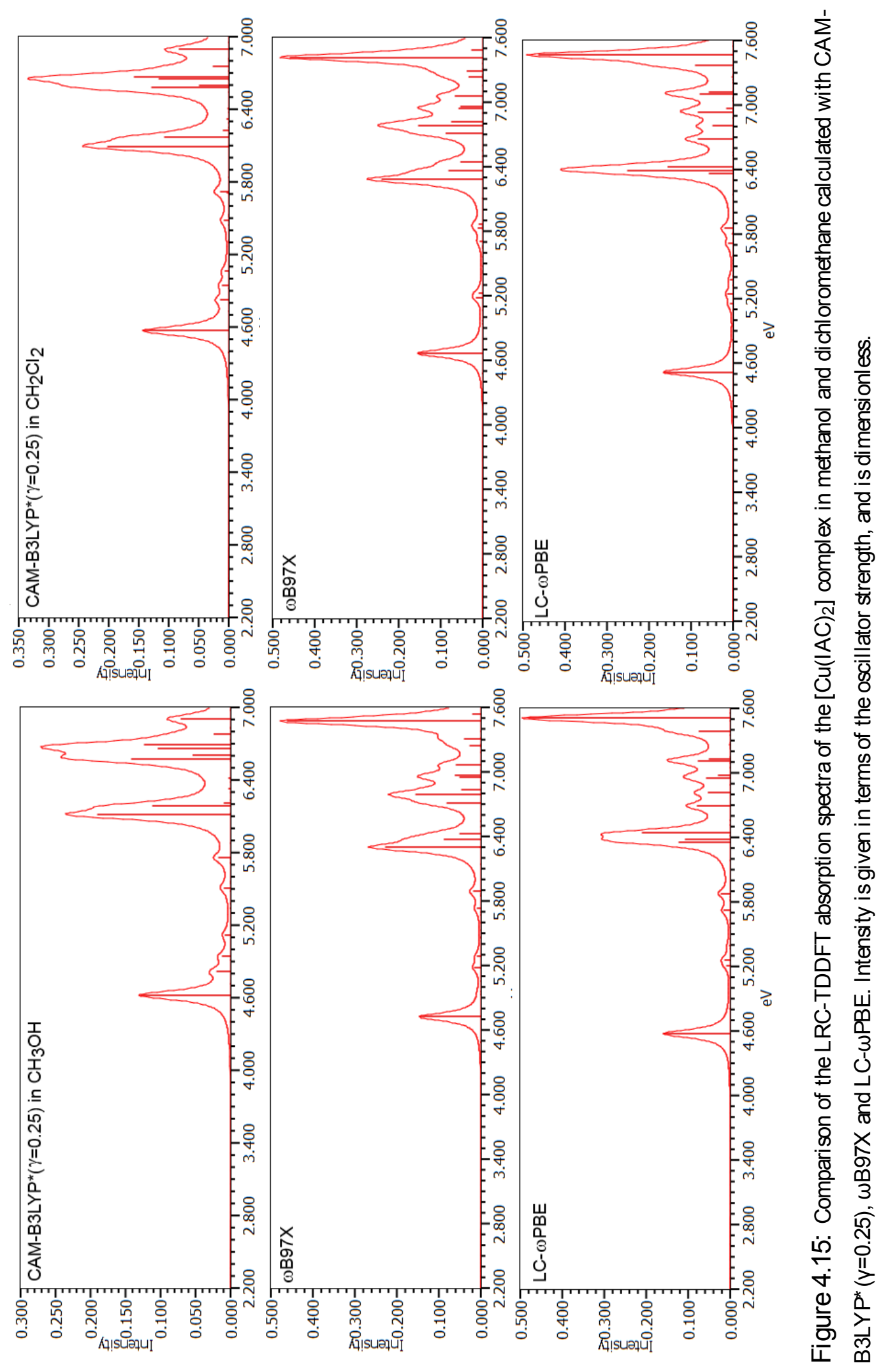




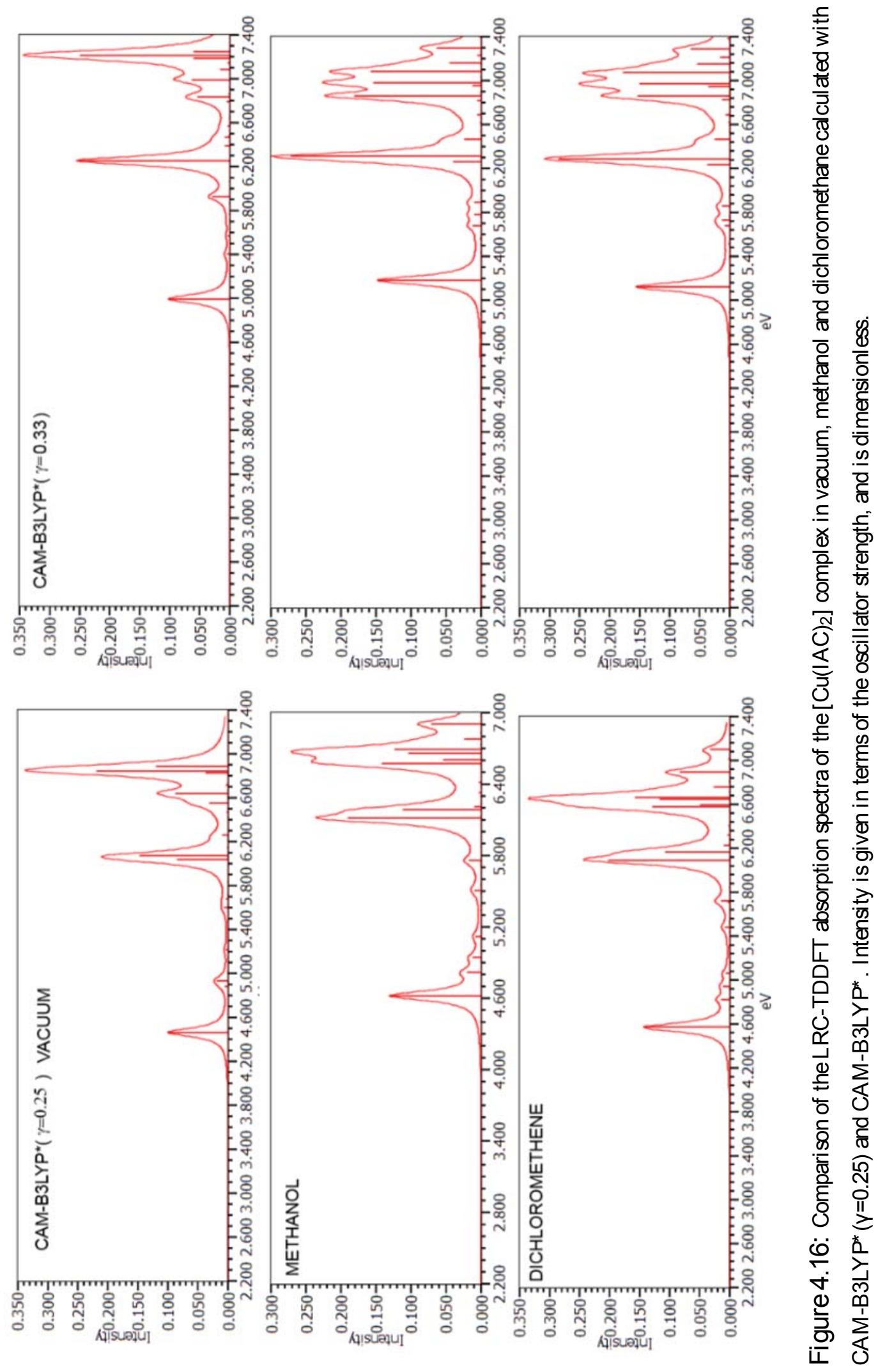


Perhaps then solvatochromic shifts are sensitive to this parameter. In fact, both $\pi-\pi^{*}$ and $n-\pi^{*}$ transitions discussed by Oliviera et al. ${ }^{156}$ shift much less with B3LYP (19\% of HF exchange) than with BHandHLYP ( $50 \%$ of HF exchange) functional. Hence it is possible that what we are claiming, i.e., $Y$ does not affect solvatochromic shifts, is correct. If that is the case we think that difference in the magnitude of solvatochromic shift between CAMB3LYP and LC- $\omega P B E$ observed by those authors ${ }^{156}$ may be due to different fraction of the SR HF exchange. Although, at the same time this assumption can be wrong as pyridinium$\mathrm{N}$-phenoxide betaine dye is very different from $\left[\mathrm{Cu}(\mathrm{IAC})_{2}\right]$, and we al ready shown that effect of y parameter on UV-Vis spectrum is highly system dependent. Unfortunately, to the best of our knowledge studies on solvatochromic shifts using LRC functionals for $\mathrm{Cu}(\mathrm{II})$ complexes ligands similar to ours are not avai lable in the literature. Further studies will be necessary to reach definite conclusions. Cal culations with LRC-TDDFT indicate that the $\left[\mathrm{Cu}(\mathrm{IAC})_{2}\right]$ complex spectrum is rich in LMCT transitions, however because of high spin contamination and highly mixed character of most of these transitions it was impossible to deduce their character. Only one of the LMCT transitions (i.e., $\sigma$ (O-carboxylate) $\rightarrow d_{x^{2}-y^{2}}$ $\mathrm{Cu}(\mathrm{II}))$ was found to be consistently pure doublet-to-doublet one electron transition; its nature was determined via TDDFT calculations. The $\sigma\left(\mathrm{O}\right.$-carboxylate) $\rightarrow d_{x^{2}-y^{2}} \mathrm{Cu}(\mathrm{II})$ transition is assigned to the experimentally determined peak with maximum absorption at $260 \mathrm{~nm}$ for measurements in methanol and $276 \mathrm{~nm}(4.77 \mathrm{eV})$ in dichloromethane. ${ }^{120}$

As mentioned before both experimental spectra show only two absorption bands; the first assigned as $\mathrm{d}-\mathrm{d}$ and second as LMCT transition. Therefore, based on our theoretical results as well as on the assignments for other similar $\mathrm{Cu}(\mathrm{II})$ complexes, ${ }^{123,124}$ the most important LMCT single electron excitation transition for the $\left[\mathrm{Cu}(\mathrm{IAC})_{2}\right]$ complex seems ti be $\sigma\left(\mathrm{O}-\right.$ carboxylate) $\rightarrow d_{x^{2}-y^{2}} \mathrm{Cu}(\mathrm{II})$. Consequently the error analysis will be discussed only for this LMCT transition. The absolute error for this transition obtained in methanol with CAM-B3LYP* $(y=0.25)$ is reduced by $0.15 \mathrm{eV}$ in comparison to theoretical data obtained in vacuum $(0.30 \mathrm{eV})$. Similarly, the accuracy of the predicted LMCT transition in 
methanol improves when both $\omega B$ 97X and LC- $\omega$ PBE functionals are utilized. In fact, results produced by $\omega B 97 X$ in $\mathrm{CH}_{3} \mathrm{OH}$ are promising because both $\mathrm{d}-\mathrm{d}$ and LMCT absorbtion peaks found experimentally are predicted with astonishing accuracy, i.e., with an error of $0.2 \mathrm{eV}$ and virtually $\mathrm{OeV}$, respectively. However, in the case of dichloromethane the performance of this functional is not as good. Mainly, al though the error for the $\mathrm{d}-\mathrm{d}$ transition is reduced in comparison to results obtained in vacuum, the inaccuracy of the predicted LMCT transition increased. For both CAM-B3LYP* $(\gamma=0.25)$ and LC- $\omega P B E$ functionals calculations that included solvation effect of $\mathrm{CH}_{2} \mathrm{Cl}_{2}$ improves the accuracy with respect to resul ts obtained in vacuum. For example, the error for predicted excitation energies of both $d-d$ and LMCT transitions ( $\sigma(\mathrm{O}-$ carboxylate $\left.) \rightarrow d_{x^{2}-y^{2}} \mathrm{Cu}(\mathrm{II})\right)$ with the CAM-B3LYP* $(Y=0.25)$ functional is about $0.1 \mathrm{eV}$; this shows the chemical accuracy of $\pm 0.1 \mathrm{eV}$ for excitation energies ${ }^{157}$ can be achieved using LRC functionals and including solvation effects. Furthermore, it is known that theoretical approaches perform better with less polar solvents. ${ }^{158}$

Somewhat surprisingly, using CAM-B3LYP* in methanol reduces the accuracy of predicted excitation energy of the $\sigma$ (O-carboxylate) $\rightarrow d_{x^{2}-y^{2}} \mathrm{Cu}(\mathrm{II})$ transition computed in vacuum. Previously this LMCT transition was found at $248 \mathrm{~nm}(5.0 \mathrm{eV})$, and in methanol at $239 \mathrm{~nm}(5.2 \mathrm{eV})$ increasing the error by $0.2 \mathrm{eV}$. Furthermore, since once the solvation effect is included the spin contamination is smaller the argument about a possible mixing of two spin states can be ruled out. Also, in dichloromethane (which is the less polar solvent among the two considered herein) CAM-B3LYP* performs worse than its variant with $Y=0.25$. Specifically, in predicting the LMCT $\left(\sigma(O-\right.$ carboxylate $\left.) \rightarrow d_{x^{2}-} y^{2} \mathrm{Cu}(I I)\right)$ transition of the $\left[\mathrm{Cu}(\mathrm{IAC})_{2}\right]$ complex. The loss in accuracy is not fully understood, although few educated guesses can be made. First of all, the val ue of the range-separation parameter in CAM-B3LYP* (i.e., $Y=0.33$ ) may be too large in the first place; in fact, with larger $Y$ the LRC is "switched on" earlier, hence introducing too much HF exchange. Another important aspect is that in real situations the metal center of the complex interacts with the 
O atom of methanol molecules trapped in the crystal. Such explicit interactions are not included in our model. Lastly, despite all the challenges that electronic structure theory still presents and that this work focused on, CAM-B3LYP* does reproduce experimental excited state properties of open-shell systems involving both transition metal and ligands with delocalized electrons ${ }^{120,123}$ quite well.

\subsection{Conclusions}

Asymptotically corrected CAM-B3LYP (noted as CAM-B3LYP*) functional has been employed to provide a consistent description of both metal- and ligand-based transitions for the $\mathrm{Cu}(\mathrm{II})$ complex with imidazole-4-acetate. The electronic spectrum of this open-shell complex is particularly challenging to study with a method based on a single-electron excitations. In fact it has been shown that the adiabatic time-dependent density functional theory (TDDFT) fails to describe excited states with significant contributions from doubleor higher electron excitations. ${ }^{132}$ Hence we focused on $\beta$-spin one-electron excitations from a doubly occupied to a singly occupied molecular orbital (so-called type 2), which can be well described with the linear response provided by TDDFT. ${ }^{145,159}$ In particular, we addressed issues regarding the appropriate choice of the range-separation parameter $(\mathrm{V})$ and fractions of exact exchange $(\alpha)$ by comparing performance of CAM-B3LYP* utilizing various values of $y$ and $\alpha$. We examined the lowest unoccupied and the highest occupied as well as few deeper molecular orbitals of different character in order to determine reasons behind occurring changes in resulting electronic spectra. Our findings suggest that: i) imbalanced treatment of orbital s of different nature takes place, ii) a more accurate agreement with experimental data ${ }^{123}$ for $\mathrm{d}-\mathrm{d}$ transitions with SOMO orbital with higher percentage of Cu-d can be expected, iii) $\Delta \mathrm{Cu}-\mathrm{d} \%$ is not reliable enough to indicate if a position of a given $d-d$ transition will shift or not upon the change of $y$ and/or a parameter utilizing the LRC hybrid functionals, iv) a more advanced method is necessary to achieve an accurate 
qual itative and quantitative description of the entire spectrum of $\left[\mathrm{Cu}(\mathrm{IA})_{2}\right]$, specifically for the peaks that possibly arose from doubly excited states, v) it is possible to achieve chemical accuracy in predicting excitation energies $(0.1 \mathrm{eV})$ of both metal- and ligand- based single-electron transitions for the UV-Vis spectrum of the $\left[\mathrm{Cu}(\mathrm{IAC})_{2}\right]$ complex. ${ }^{120}$ using $C A M-B 3 L Y P^{*}(\gamma=0.25)$ while including the environment of the non-polar solvent. 


\section{Chapter 5}

\section{Influence of Basis Set for Predicting \\ Acidities of First-Row Hydrides and their Lithiated Analogs ${ }^{1}$}

\subsection{Introduction}

One of the chal lenges in computational chemistry is the choice of basis set, that is a set of mathematical functions used to describe the wave-like behavior of electrons in atomic and molecular systems. Each basis function is usually constructed as a linear combination (contraction) of Gaussian functions, called primitives, that form Slater-like functions, HF atomic orbitals or other set of functions. ${ }^{5}$ Note that the least costly SCF HF method requires cal culations which scale as $\mathrm{N}^{4}$, where $\mathrm{N}$ is the number of basis functions. Thus, from a practical point of view one may want to employ post-HF methods within a minimal basis set (STO-3G), that is basis set which includes only functions present at a given atomic

\footnotetext{
${ }^{1}$ The material contained in this Chapter will be submitted to Int. J. Quantum Chem. (2013) ${ }^{165}$
} 
shell and/or are necessary to describe the occupied atomic orbitals of the element. For example, Li has three electrons which occupy 1s and 2s orbital s but STO-3G for this atom has 5 basis functions $1 \mathrm{~s}, 2 \mathrm{~s}$ and $2 \mathrm{p}$, al though the $\mathrm{p}$ orbitals are not occupied. On the other hand to achieve meaningful results one has to often use much greater basis set than STO-3G, this can be done by increasing the number of functions. For instance, if one doubles their number this will all ow to construct a double zeta $(\zeta)$ basis set. Other common basis sets are so-cal led split valence, where only functions that bel ong to the valance shell are doubled. The next step to improve a basis set would be to go beyond double $\zeta$, so by building triple, quadruple, etc. basis set. However, it is important to maintain the balance of the basis set, i.e., in addition to increasing the number of $s$ and $p$ basis functions one must include al so basis functions with a higher angular momentum (e.g., $d$ functions). Otherwise the luck of such basis functions may cause an artificial results.

The most accurate results can be achieved within the so-called HF limit, i.e., a basis set containing an infinite number of basis functions, but obviously such basis set is impossible to construct. Nonethel ess, in some cases it is possi ble to extrapolate results from the finite basis set to the HF limit. 4,5

Modern basis sets such as these developed by Pople (e.g., 6-31G, 6-31G* , 6-31G* , 6$311 G^{*}$, etc.) ${ }^{160,161}$ or by Dunning (cc-pVXZ and aug-cc-pVXZ) $)^{160,161}$ make a popular choice. Nevertheless even with limiting the choice of the basis sets to these two families it is crucial to select a basis set that would be the most efficient and provide the most accurate result for a considered chemical problem. In this study the dependency of the most popular computational methods on the choice of the basis set will be shown for the acidity $\left(\Delta \mathrm{H}_{\text {acid }}^{\circ}\right)$ trend of the first-row hydrides, specifically, for lithium hydroxide ( $\left.\mathrm{LiOH}\right)$ and methane $\left(\mathrm{CH}_{4}\right)$. The main goal of this study was to prove that augmented basis sets are necessary to obtain the correct order of acidities, i.e., $\Delta \mathrm{H}_{\text {acid }}^{298 \mathrm{~K}}\left(\mathrm{CH}_{4}\right)<\Delta \mathrm{H}_{\text {acid }}^{298 \mathrm{~K}}(\mathrm{LiOH})$ independently from the theory. Therefore, different types of methods were employed; a 
wave-function approach, i.e., $\operatorname{CCSD}(\mathrm{T})$ as an example of the coupled-cluster methodology and two approaches based on the electron density. The latter belong to the family of GGA functionals; the BLYP is a pure DFT functional and the B3LYP is taken as an example of a hybrid functional. Tested basis sets were Dunning's: ${ }^{162}$ cc-pVDZ, cc-pVTZ, aug-cc-pVDZ and aug-cc-pVTZ; and Pople' s: ${ }^{163,164} 6-31 G^{* *}, 6-31+G^{* *}, 6-311 G^{* *}$ and $6-311+G^{* *}$ (also known as 6-31G(d,p), 6-31+G(d,p), etc.)

This idea has been inspired by the work of Tian et al. ${ }^{166}$ who first presented a study on the acidity of lithium monoxide anion, where they were able to generate the gas phase lithium monoxide anion ( $\mathrm{LiO}^{-}$) which was found to be the strongest base. In fact, it was proved by both the high level ab initio cal culations and high quality experimental measurements that $\mathrm{LiO}^{-}$is more basic than methyl anion $\left(\mathrm{CH}_{3}^{-}\right)$, known as the most basic specie for about thirty years before this discovery. Although, Tian's group achieved very good agreement between their best theoretical result $\left(\Delta \mathrm{H}_{\text {acid }}^{298 \mathrm{~K}}(\mathrm{LiOH})=426.2 \pm 2 \mathrm{kcal} / \mathrm{mol}\right)$ and the experimental value $(425.7 \pm 6.1 \mathrm{kcal} / \mathrm{mol})$ the rol e of aug-cc-pVQZ ${ }^{162}$ basis set was not discussed. Furthermore, both computational methods used in the study, viz $\operatorname{BD}(T)^{167,168}$ and the Complete Active Space Averaged Quadratic Coupled Cluster ${ }^{169,170}$ (CAS-AQCC), are not a common a choice in computational chemistry. The first due to less popular Brueckner reference determinant, the second because of its high computational cost and expertise needed from the user to correctly conduct cal culations with this method. For this reason in this study $\operatorname{CCSD}(T)$ approach has been employed as an example of highly accurate "black box" method, that can be applied to predict the acidities $\left(\Delta \mathrm{H}_{\text {acid }}^{298 \mathrm{~K}}\right)$ of first-row hydrides by non-experts. 


\subsection{Method}

The calculations were performed using the Gaussian $03^{142}$ program, where geometries were optimized within the given method and basis set, unless stated differently. In case of open-shell systems a default set-up in this software employs the UHF reference wave function for $\operatorname{CCSD}(T)$ method and the unrestricted spin-density for DFT functional s(i.e., UBLYP, UB3LYP). Note that the unrestricted approaches tend to contaminate spin states. ${ }^{171}$ Therefore, in order to ensure that the correct acidity trend was not obtained artificially, additional calculations were done using the restricted open-shell approach (i.e., ROHF) for $\operatorname{CCSD}(T)$ method with cc-pVDZ and aug-cc-pVDZ basis sets using the NWChem $6.0^{172,173}$ program. No significant difference between using the UHF and ROHF reference was noticed, for example, the CCSD(T)/cc-pVDZ cal culations the $\Delta \mathrm{H}_{\text {acid }}\left(\mathrm{X}^{\mathrm{ROHF}}\right)-\Delta \mathrm{H}_{\text {acid }}\left(\mathrm{X}^{\mathrm{UHF}}\right)$ is only $0.3 \mathrm{kcal} / \mathrm{mol}$.

The gas-phase Proton Affinity (PA) is often used as a measure of basicity for the neutral molecule or an anion and for the schematic reaction of protonation/deprotonation such as $\mathrm{A}^{-}+\mathrm{H}^{+} \rightarrow \mathrm{HA}$ the PA can be determined as the negative of the heat of reaction at $298 \mathrm{~K}$ :

$$
P A=-\Delta H_{r x n}=-\Delta E_{r x n}+R T
$$

where the $\mathrm{R}$ is ideal-gas constant and $\mathrm{T}$ is temperate.

The total energy of the polyatomic molecule may be approximated as follows:

$$
E_{t} \equiv E_{e l}+Z P E+E_{r o t}+E_{\text {trans }}+E_{t h e r m-v i b}
$$

The first two terms are the electronic energy and thezero-point vibrational energy of agiven specie, respectively. The $E_{\text {rot }}$ is the rotational energy and $E_{\text {trans }}$ is translation energy, which from the statistical mechanics are known to be equal to $3 / 2 R T$. The next term $E_{\text {therm-vib }}$ is expressed as shown below.

$$
E_{\text {therm- vib }}=N_{A} \sum_{k=1}^{3 N-6} \frac{h v_{k}}{\left[e\left(\frac{h v_{k}}{k_{b} T}\right)-1\right]} \text { non- linear }
$$


Note that for a linear molecule the the $E_{\text {rot }}$ and $E_{\text {thermal-vib }}$ are defined differently, i.e., the contribution of the former is equal to RT and

$$
E_{\text {therm- vib }}=N_{A} \sum_{k=1}^{3 N-5} \frac{h v_{k}}{\left[e\left(\frac{h v_{k}}{k_{b} T}\right)-1\right]} \text { linear }
$$

where $\mathrm{h}$ is Plank's constant, $\mathrm{k}_{\mathrm{b}}$ is the Boltzmann's constant, $\mathrm{T}$ is temperature and $\mathrm{v}_{\mathrm{k}}$ indicates frequency of $k^{\text {th }}$ normal mode in molecule.

The energetic contributions from $E_{\text {therm- vib }}$ are usually negligible compare to ZPE, the $E_{\text {rot }}$ and $E_{\text {trans }}$ contributions either maintain constant (if geometry of the molecule would change by removing/adding proton from linear to non-linear and vice-versa) or cancel out, because we need the relative energy for obtaining values of proton affinity. Hence in order to predict the gas-phase PA at $298 \mathrm{~K}$ theoretically one has to calculate the electronic energies of the neutral and anionic forms of the considered molecule, include the difference between their ZPEs and the translational energy of the proton, which is 3/2RT (note that proton does not have rotational kinetic energy and $E_{e l e}$ contribution is zero). So the final equation used to obtain the PA at $298 \mathrm{~K}$ is given as follows:

$$
\mathrm{PA}^{298 \mathrm{~K}}=\Delta \mathrm{E}_{\mathrm{ele}}+\Delta \mathrm{ZPE}+5 / 2 \mathrm{RT}
$$

where

$$
\Delta E_{e l e}=E_{e l e}\left(A^{-}\right)-E_{e l e}(H A)
$$

and

$$
\Delta \mathrm{ZPE}=\mathrm{ZPE}\left(\mathrm{A}^{-}\right)-\mathrm{ZPE}(\mathrm{HA})
$$

Note that higher value of PA indicates a weaker acid (or a stronger base) and from now on the $\mathrm{PA}^{298 \mathrm{~K}}=\Delta \mathrm{H}_{\mathrm{acid}}^{298 \mathrm{~K}}$ and its value at $\mathrm{OK}$ is equivalent to $\Delta \mathrm{E}_{\mathrm{ele}}$, which will be noted as $\Delta \mathrm{H}_{\text {acid. }}$. The reason why we are al lowed to use Eq. (5.5) is that for all molecules except of $\mathrm{LiNH}_{2} / \mathrm{LiNH}^{-}$the geometrical arrangement of atoms is the same, i.e., either non-linear or linear (see Fig. 5.2 and Fig. 5.3). Therefore the energetic contributions from $E_{\text {rot }}$ and $E_{\text {trans }}$ of these species cancels out. The PA ${ }^{298}$ changes slightly for $\mathrm{LiNH}_{2} / \mathrm{LiNH}^{-}$, because LiNH is non-linear whereas LiNH is, so instead of 5/2RT we need to use $2 \mathrm{RT}$ due to difference between 5/ 2RT + [( $\left.\left.\mathrm{E}_{\text {rot }}\left(\mathrm{LiNH}^{-}\right)\right)-\left(\mathrm{E}_{\text {rot }}\left(\mathrm{LiNH}_{2}\right)\right)\right]$. 


\subsection{Results and Discussion}

The results presented in this work are organized as follows:

† Set 1: BLYP, B3LYP and CCSD(T) cal culations employing Dunning's basis sets (Table 5.1 and Table 5.2);

† Set 2: BLYP, B3LYP calculations employing Pople's basis sets (Table 5.3 and Table 5.4);

$\dagger$ Test 1: Acidities of first-row hydrides at CCSD(T)/aug-cc-pVDZ//MP2/aug-cc-pVTZ level versus theoretical predictions of Tian's group (Fig 5.1);

† Test 2: The CCSD method using Dunning's basis set is compared to resul ts obtained via $\operatorname{CCSD}(\mathrm{T})$ (Table 5.5);

† Test 3: The structures and energies of the lowest singlet state and the lowest triplet states of neutral and anionic molecular systems of the first-row hydrides were computed (Fig 5.2 and Fig 5.3).

For all methods values of electronic energies, presented in Table (5.1), decrease with the increase of the size of basis set, so $E_{e l}^{C C D Z}>E_{e l}^{A C C D}>E_{e l}^{C C T Z}>E_{e l}^{A C C T}$, where CCDZ, ACCD, CCTZ and ACCT standsfor cc-pVDZ, aug-cc-pVDZ, cc-pVTZ, and aug-cc-pVTZ, respectively. This is not surprising, in fact it shows a proper behavior of BLYP, B3LYP and $\operatorname{CCSD}(T)$ methods with Dunning's basis sets, but does the lowering of electronic energies necessarily mean obtaining the proper ordering of acidities? Furthermore, how does this affect anionic and neutral molecules? Is it possible to identify any trend? 
Since the last question is the easiest to be answered, we shell start from it. The following trend can be identified $\Delta \mathrm{H}_{\text {acid }}^{(\mathrm{CCDZ})}>\Delta \mathrm{H}_{\text {acid }}^{(\mathrm{ACCD})}$ and $\Delta \mathrm{H}_{\text {acid }}^{(\mathrm{CCTZ})}>\Delta \mathrm{H}_{\text {acid }}^{(\mathrm{ACCT})}$, where the acidity decreases with the improving of the basis set. The most noticeable change can be observed for acidities of methane, when switching from cc-pVDZ [H:2s1p, non- $\mathrm{H}: 3 \mathrm{~s} 2 \mathrm{p} 1 \mathrm{~d}]$ to aug-cc-pVDZ [H:3s2p, non-H:3s3p2d] basis set. In fact, the decrease of $\Delta \mathrm{H}_{\text {acid }}$ values is in the range of $24-34 \mathrm{kcal} / \mathrm{mol}$. This change is not as significant between cc-pVTZ [H:3s2p1d, non-H:4s3p2d1f] and aug-cc-pVTZ [H:4s3p2d, non-H:5s4p3d2f] basis sets, i.e., about $12-21 \mathrm{kcal} / \mathrm{mol}$. This can be explained as due to additional diffuse functions on hydrogen and on first-row el ements that have much greater effect than the addition of polarization functions on these atoms. $\Delta \mathrm{H}_{\text {acid }}$ for the $\mathrm{LiOH}$ obtained at different levels are much less influenced, which seems to be related to both previous explanations. Consequently, the decrease in $\Delta \mathrm{H}_{\text {acid }}(\mathrm{LiOH})$ is in the range of 3-5 kcal/mol for both cc-pVDZ/aug-cc-pVDZ and cc-pVTZ/aug-cc-pVTZ.

The first question is directly connected to the second one. To answer both the energy change $(\Delta \mathrm{E})$ with respect to the "transition" between cc-pVXZ and the corresponding augmented basis set, the type of molecule and the computational method were analyzed. The energy changes for all basis sets at all methods are significantly bigger for ions than for neutral molecules, this can be attributed to the fact that the electron added to induce negatively charged species is noncovalenlty bound and as such requires diffused functions (which thanks to their long tails can have electrons in farther distance form nuclei) for its proper description. ${ }^{174}$ For example, the energy difference for $\mathrm{CH}_{3}^{-}$obtained with BLYP/cc-pVDZ and BLYP/aug-cc-pVDZ equals $38 \mathrm{kcal} / \mathrm{mol}$ while for $\mathrm{CH}_{4}$ (neutral) only about $4 \mathrm{kcal} / \mathrm{mol}$. A similar pattern can be noticed for other methods as well as the cc-pVTZ/aug-cc-pVTZ pair, even though the $\Delta \mathrm{Es}$ of $\mathrm{LiO}^{-}$and $\mathrm{LiOH}$ are less influenced than those for $\mathrm{CH}_{3}^{-}$and $\mathrm{CH}_{4}$. This gives us an indication about the relationship between $\Delta \mathrm{E}$ and $\Delta \mathrm{H}_{\text {acid }}$ that is mostly ruled by the choice of the basis set. 
As one can see in Table 5.1 for cal culations at OK using BLYP and CCSD(T) methods with Dunnig's basis set the augmentation of cc-pVDZ basis set was enough to recover proper acidity trend. However, in case of B3LYP we need to augment a bigger basis set (cc-pVTZ) in order to archive the same goal. If we look more closely, the acidity of methane has been lowered by about $31 \mathrm{kcal} / \mathrm{mol}$ and the acidity of lithium hydroxide about $3 \mathrm{kcal} / \mathrm{mol}$. This results are somewhat imbalanced compared to the effects reported for other methods.

Table 5.1: Cal culated Energies and Acidities $\left(\Delta \mathrm{H}_{\text {acid }}\right)$ in $\mathrm{kcal} / \mathrm{mol}$ using Dunning's basis sets at $0 \mathrm{~K}$.

\begin{tabular}{|c|c|c|c|c|}
\hline BLYP & cc-pVDZ & aug-cc-pVDZ & cc-pVTZ & aug-cc-pVTZ \\
\hline $\mathrm{LiO}^{-}$ & -51882.4 & -51896.9 & -51902.6 & -51909.8 \\
\hline $\mathrm{LiOH}$ & -52310.5 & -52320.9 & -52333.8 & -52336.4 \\
\hline$\Delta \mathrm{H}_{\text {acid }}$ & 428.1 & 424.0 & 431.2 & 426.6 \\
\hline $\mathrm{CH}_{3}^{-}$ & -24943.9 & -24981.8 & -24971.9 & -24992.8 \\
\hline $\mathrm{CH}_{4}$ & -25399.0 & -25402.7 & -25414.6 & -25414.8 \\
\hline$\Delta \mathrm{H}_{\text {acid }}$ & 455.1 & 420.9 & 442.7 & 421.9 \\
\hline B3LYP & cc-pVDZ & aug-cc-pVDZ & cc-pVTZ & aug-cc-pVTZ \\
\hline $\mathrm{LiO}^{-}$ & -51896.6 & -51910.0 & -51915.9 & -51922.1 \\
\hline $\mathrm{LiOH}$ & -52324.5 & -52334.5 & -52346.6 & -52349.0 \\
\hline$\Delta \mathrm{H}_{\text {acid }}$ & 427.9 & 424.6 & 430.7 & 426.9 \\
\hline $\mathrm{CH}_{3}^{-}$ & -24968.1 & -25001.8 & -24993.5 & -25011.8 \\
\hline $\mathrm{CH}_{4}$ & -25424.4 & -25427.1 & -25438.1 & -25438.2 \\
\hline$\Delta \mathrm{H}_{\text {acid }}$ & 456.3 & 425.3 & 444.7 & 426.5 \\
\hline $\operatorname{CcsD}(T)$ & cc-pVDZ & aug-cc-pVDZ & cc-pVTZ & aug-cc-pVTZ \\
\hline $\mathrm{LiO}^{-}$ & -51742.9 & -51766.6 & -51795.4 & -51805.0 \\
\hline $\mathrm{LiOH}$ & -52170.3 & -52190.6 & -52227.8 & -52234.2 \\
\hline$\Delta \mathrm{H}_{\text {acid }}$ & 427.4 & 423.9 & 432.4 & 429.1 \\
\hline $\mathrm{CH}_{3}^{-}$ & -24895.7 & -24925.4 & -24936.8 & -24951.6 \\
\hline $\mathrm{CH}_{4}$ & -25343.6 & -25348.7 & -25374.9 & -25377.0 \\
\hline$\Delta \mathrm{H}_{\text {acid }}$ & 447.9 & 423.3 & 438.1 & 425.4 \\
\hline
\end{tabular}


For BLYP the difference between $\Delta \mathrm{H}_{\text {acid }}(\mathrm{LiOH}) \mathrm{s}$ is bigger and so the $\Delta \mathrm{H}_{\text {acid }}\left(\mathrm{CH}_{4}\right) \mathrm{s}$, in case of $\operatorname{CCSD}(\mathrm{T})$ a smaller difference for $\mathrm{LiOH}$ match less reduced acidities of $\mathrm{CH}_{4}$. B3LYP lowers $\Delta \mathrm{H}_{\text {acid }}(\mathrm{LiOH})$ less than $\Delta \mathrm{H}_{\text {acid }}\left(\mathrm{CH}_{4}\right)$. Thus since the $\Delta \mathrm{H}_{\text {acid }}$ values are obtained from the energy difference between $\mathrm{LiO}^{-}$and $\mathrm{LiOH}$ (see Eq. (5.6)), the cancellation of errors is not effective enough at the B3LYP/aug-cc-pVDZ level of theory. This is due to the luck of sufficient amount of corrected energy for $\mathrm{LiO}^{-} / \mathrm{LiOH}$ pair with aug-ccpVDZ basis set for this method, resulting into the improper acidity order. Results presented in Table 5.2 include the ZPE and thermal corrections by addition of $\triangle Z P E+5 / 2 R T$ or $2 R T$ (see section 5.2)to previously cal culated acidities (i.e., at OK). The vibrational zero point energies are obviously also affected by the choice of a basis set but somewhat less consistently than it is for energies or $\Delta \mathrm{H}_{\text {acid }} \mathrm{S}$.

First one can notice that ZPECCDZ $>$ ZPEACCD and ZPE ${ }^{C C T Z}>$ ZPEACCT for $\mathrm{LiO}^{-}$and $\mathrm{LiOH}$ for all methods but $\mathrm{B} 3 \mathrm{LYP}$, where $Z \mathrm{PE}{ }^{\mathrm{CCDZ}}<\mathrm{ZPE}{ }^{\mathrm{ACCD}}$. For the methane molecule $Z P^{C C D Z}>Z P E^{A C C D}$ and $Z P E^{C C T Z}>Z P E^{A C C T}$ for all methods except $C C S D(T)$, where $Z P E^{C C D Z}<Z P E^{A C C D}$ and $Z P E^{C C T Z} \cong Z P E^{A C C T}$. In the case of the methane anion $Z P^{C C D Z}<Z P E^{A C C D}$ and $Z P E^{C C T Z}<Z P^{A C C T}$ for all methods, but within TZ basis sets the last trend is reversed for $\operatorname{CCSD}(T)$. Although these differences in trends may at first seem not to play a role in the acidity pattern, now calculations at B3LYP/aug-cc-pVDZ theory level give the correct acidity order $\left(\Delta \mathrm{H}_{\text {acid }}^{298 \mathrm{~K}}(\mathrm{LiOH})>\Delta \mathrm{H}_{\text {acid }}^{298 \mathrm{~K}}\left(\mathrm{CH}_{4}\right)\right)$. The influence of $\Delta \Delta Z \mathrm{ZPE}{ }^{\mathrm{X}}$, where $\mathrm{X}=\mathrm{DZ}$ or $\mathrm{TZ}$ (i.e., $\Delta \Delta Z \mathrm{PE}^{\mathrm{DZ}}=\Delta \mathrm{ZPE} \mathrm{ACCD}^{-} \triangle \mathrm{ZPE} \mathrm{CCDZ}^{\mathrm{C}}$ or $\left.\Delta \triangle Z \mathrm{PE}^{\mathrm{TZ}}=\Delta \mathrm{ZPE} \mathrm{ACCT}^{\mathrm{A}}-\Delta \mathrm{ZPE}^{\mathrm{CCT}}\right)$, on changes of $\Delta \mathrm{H}_{\text {acid }}^{298 \mathrm{~K}}(\mathrm{LiOH})$ values employing $B L Y P$ and $C C S D(T)$ methods are indeed very small, yet for B3LYP $\triangle \triangle Z P E{ }^{D Z}$ is greater. Hence, by including $\triangle \mathrm{ZPE}$ one can change the qual itative picture of results obtained in $0 \mathrm{~K}$. 
Table 5.2: Cal culated Zero Point Energies (ZPE) and Acidities $\left(\Delta \mathrm{H}_{\text {acid }}^{298 \mathrm{~K}}\right)$ in $\mathrm{kcal} / \mathrm{mol}$ using Dunning's basis sets.

\begin{tabular}{|c|c|c|c|c|}
\hline BLYP & cc-pVDZ & aug-cc-pVDZ & cc-pVTZ & aug-cc-pVTZ \\
\hline ZPE( $\left.\mathrm{LiO}^{-}\right)$ & 1.047 & 0.937 & 0.978 & 0.962 \\
\hline ZPE( $\mathrm{LiOH})$ & 6.949 & 6.607 & 6.813 & 6.615 \\
\hline$\triangle \mathrm{ZPE}$ & -5.902 & -5.670 & -5.836 & -5.653 \\
\hline$\Delta \mathrm{H}_{\text {acid }}^{298 \mathrm{~K}}$ & 423.7 & 419.8 & 426.8 & 422.4 \\
\hline $\mathrm{ZPE}\left(\mathrm{CH}_{3}^{-}\right)$ & 16.607 & 17.071 & 16.883 & 17.172 \\
\hline $\mathrm{ZPE}\left(\mathrm{CH}_{4}\right)$ & 27.131 & 27.086 & 27.338 & 27.301 \\
\hline$\Delta \mathrm{ZPE}$ & -10.524 & -10.015 & -10.456 & -10.129 \\
\hline$\Delta \mathrm{H}_{\mathrm{acid}}^{298 \mathrm{~K}}$ & 446.0 & 412.3 & 433.7 & 413.3 \\
\hline B3LYP & cc-pVDZ & aug-cc-pVDZ & cc-pVTZ & aug-cc-pVTZ \\
\hline ZPE(LiO- ) & 1.053 & 0.975 & 1.010 & 0.997 \\
\hline ZPE( $\mathrm{LiOH})$ & 7.228 & 7.964 & 7.036 & 6.835 \\
\hline$\triangle \mathrm{ZPE}$ & -6.175 & -6.990 & -6.026 & -5.838 \\
\hline$\Delta H_{\text {acid }}^{298 K}$ & 423.3 & 419.1 & 426.1 & 422.6 \\
\hline $\mathrm{ZPE}\left(\mathrm{CH}_{3}^{-}\right)$ & 17.066 & 17.492 & 17.293 & 17.571 \\
\hline $\mathrm{ZPE}\left(\mathrm{CH}_{4}\right)$ & 27.822 & 27.753 & 27.983 & 27.956 \\
\hline$\triangle \mathrm{ZPE}$ & -10.756 & -10.260 & -10.690 & -10.385 \\
\hline$\Delta \mathrm{H}_{\text {acid }}^{298 \mathrm{~K}}$ & 447.0 & 416.5 & 435.4 & 417.6 \\
\hline $\operatorname{CCSD}(T)$ & cc-pVDZ & aug-cc-pVDZ & cc-pVTZ & aug-cc-pVTZ \\
\hline ZPE(LiO- ) & 1.043 & 0.950 & 1.002 & 0.985 \\
\hline ZPE( $\mathrm{LiOH})$ & 8.093 & 7.881 & 8.045 & 7.794 \\
\hline$\triangle \mathrm{ZPE}$ & -7.050 & -6.931 & -7.043 & -6.809 \\
\hline$\Delta \mathrm{H}_{\text {acid }}^{298 \mathrm{~K}}$ & 421.8 & 418.5 & 426.8 & 423.8 \\
\hline $\mathrm{ZPE}\left(\mathrm{CH}_{3}^{-}\right)$ & 17.417 & 17.659 & 17.654 & 17.390 \\
\hline $\mathrm{ZPE}\left(\mathrm{CH}_{4}\right)$ & 28.114 & 27.845 & 28.115 & 28.117 \\
\hline$\triangle \mathrm{ZPE}$ & -10.698 & -10.186 & -10.461 & -10.728 \\
\hline$\Delta \mathrm{H}_{\text {acid }}^{298 \mathrm{~K}}$ & 438.7 & 414.6 & 429.2 & 416.2 \\
\hline
\end{tabular}


Dunning's correlation consistent basis sets and their augmented anal ogs are sets of Gaussian functions which were optimized to work best for methods that explicitly deal with correlation effects (e.g., the coupled cluster methods). Therefore cal cul ations using Pople's basis sets for both DFT functionals were performed and the results are shown in Table 5.3 at $0 \mathrm{~K}$ and in Table 5.4 at $298 \mathrm{~K}$.

Table 5.3: Cal culated Energies and Acidities $\left(\Delta \mathrm{H}_{\text {acid }}\right)$ in $\mathrm{kcal} / \mathrm{mol}$ using Pople's basis sets at OK. No thermal or ZPE corrections have been applied.

\begin{tabular}{l|cccc}
\hline \hline $\mathrm{BLYP}$ & $6-31 \mathrm{G}^{* *}$ & $6-31+\mathrm{G}^{* *}$ & $6-311 \mathrm{G}^{* *}$ & $6-311+\mathrm{G}^{* *}$ \\
\hline $\mathrm{LiO}^{-}$ & -51873.4 & -51890.2 & -51895.6 & -51906.2 \\
$\mathrm{LiOH}$ & -52304.0 & -52313.6 & -52325.4 & -52332.5 \\
\hline$\Delta \mathrm{H}_{\text {acid }}$ & 430.7 & 423.4 & 429.9 & 426.3 \\
\hline $\mathrm{CH}_{3}^{-}$ & -24948.7 & -24984.8 & -24971.5 & -24990.7 \\
$\mathrm{CH}_{4}$ & -25404.5 & -25406.5 & -25411.8 & -25411.9 \\
\hline$\Delta \mathrm{H}_{\text {acid }}$ & 455.9 & 421.6 & 440.3 & 421.2 \\
\hline $\mathrm{B} 3 \mathrm{LYP}$ & $6-31 \mathrm{G}^{* *}$ & $6-31+\mathrm{G}^{* *}$ & $6-311 \mathrm{G}^{* *}$ & $6-311+\mathrm{G}^{* *}$ \\
\hline $\mathrm{LiO}^{-}$ & -51887.6 & -51903.1 & -51908.9 & -51918.5 \\
$\mathrm{LiOH}^{\mathrm{O} O \mathrm{H}}$ & -52318.0 & -52327.1 & -52338.4 & -52345.1 \\
\hline$\Delta \mathrm{H}_{\text {acid }}$ & 430.4 & 424.1 & 429.6 & 426.7 \\
\hline $\mathrm{CH}_{3}^{-}$ & -24972.4 & -25004.6 & -24992.9 & -25010.1 \\
$\mathrm{CH}_{4}$ & -25429.2 & -25430.5 & -25435.3 & -25435.4 \\
\hline$\Delta \mathrm{H}_{\text {acid }}$ & 456.8 & 425.9 & 442.4 & 425.3 \\
\hline \hline
\end{tabular}

Val ues of gas phase acidities at OK decrease for augmented Pople's basis sets, for example, in case of $\mathrm{LiOH} \Delta \mathrm{H}_{\text {acid }}^{\left(6-31 \mathrm{G}^{* *}\right)}>\Delta \mathrm{H}_{\text {acid }}^{\left(6-31+\mathrm{G}^{* *}\right)}$, which agrees with findings for Dunning's basis sets. Furthermore the energies lower with the increase of the size of basis set, i.e., $E_{e}^{6-31 G * *}>E_{e l}^{6-31+G^{* *}}>E_{e l}^{6-311 G^{\star *}}>E_{e l}^{6-311+G^{* *}}$, the ionic species being more affected than neutral molecules, $\Delta \mathrm{H}_{\text {acid }}^{6-31 \mathrm{G}^{* *}}>\Delta \mathrm{H}_{\text {acid }}^{6-31+\mathrm{G}^{* *}}, \Delta \mathrm{H}_{\text {acid }}^{6-311 \mathrm{G}^{* *}}>\Delta \mathrm{H}_{\text {acid }}^{6-311+\mathrm{G}^{* *}}$. In addi- 
tion, cal culations at B3LYP/6-31+G** theory level show improper acidity order.

TheZPE and thermally corrected B3LYP/6-31+ $G^{* *}$ cal culations al low to retrieve the proper acidity trend as it was in the case of B3LYP/aug-cc-pVDZ. This and other evidences discussed earlier in the text show that B3LYP functional is less reliable than BLYP and a bigger basis set has to be employed (i.e., at least triple $\zeta$ type basis set) to assure correct results using B3LYP.

Table 5.4: Cal culated Zero Point Energies (ZPE) and Acidities $\left(\Delta \mathrm{H}_{\mathrm{acid}}^{298 \mathrm{~K}}\right)$ in kcal/mol using Pople's basis sets.

\begin{tabular}{l|cccc}
\hline \hline BLYP & $6-31 G^{* *}$ & $6-31+\mathrm{G}^{* *}$ & $6-311 \mathrm{G}^{* *}$ & $6-311+\mathrm{G}^{* *}$ \\
\hline $\mathrm{ZPE}\left(\mathrm{LiO}^{-}\right)$ & 1.017 & 0.943 & 0.993 & 0.953 \\
$\mathrm{ZPE}(\mathrm{LiOH})$ & 7.231 & 7.688 & 7.935 & 7.969 \\
$\Delta \mathrm{ZPE}$ & -6.214 & -6.746 & -6.942 & -7.017 \\
\hline$\Delta \mathrm{H}_{\mathrm{acid}}^{298 \mathrm{~K}}$ & 425.9 & 418.1 & 424.4 & 420.8 \\
\hline $\mathrm{ZPE}\left(\mathrm{CH}_{3}^{-}\right)$ & 16.854 & 17.547 & 16.850 & 17.450 \\
$\mathrm{ZPE}\left(\mathrm{CH}_{4}\right)$ & 27.596 & 27.428 & 27.341 & 27.301 \\
$\Delta \mathrm{ZPE}$ & -10.742 & -9.881 & -10.491 & -9.851 \\
\hline$\Delta \mathrm{H}_{\mathrm{acid}}^{298 \mathrm{~K}}$ & 446.6 & 413.2 & 431.3 & 412.8 \\
\hline $\mathrm{B} 3 \mathrm{LYP}$ & $6-31 \mathrm{G}^{* *}$ & $6-31+\mathrm{G}^{* *}$ & $6-311 \mathrm{G}^{* *}$ & $6-311+\mathrm{G}^{* *}$ \\
\hline $\mathrm{ZPE}(\mathrm{LiO}-$ & 1.049 & 0.981 & 1.029 & 0.988 \\
$\mathrm{ZPE}(\mathrm{LiOH})$ & 7.511 & 7.980 & 8.181 & 8.224 \\
$\Delta \mathrm{ZPE}$ & -6.461 & -6.999 & -7.152 & -7.236 \\
\hline$\Delta \mathrm{H}_{\mathrm{acid}}^{298 \mathrm{~K}}$ & 425.4 & 418.6 & 423.9 & 420.9 \\
\hline $\mathrm{ZPE}\left(\mathrm{CH}_{3}^{-}\right)$ & 17.465 & 17.999 & 17.363 & 17.883 \\
$\mathrm{ZPE}\left(\mathrm{CH}_{4}\right)$ & 28.256 & 28.106 & 27.979 & 27.948 \\
$\Delta \mathrm{ZPE}$ & -10.791 & -10.107 & -10.616 & -10.065 \\
\hline$\Delta \mathrm{H}_{\text {acid }}^{298 \mathrm{~K}}$ & 447.5 & 417.3 & 433.2 & 416.8 \\
\hline \hline
\end{tabular}


Once the importance of augmentation of Dunning's and Pople's basis set in achieving the correct order of acidities for $\mathrm{LiOH}$ and $\mathrm{CH}_{4}$ was established (see discussion above and results in Tables 5.1 through 5.4), the next set of calculations was performed utilizing MP2/aug-cc-pVTZ for geometry optimization and $\operatorname{CCSD}(T) / a u g-c c-p V D Z$ for the single point energies, which will be indicated in the text below as $\operatorname{CCSD}(T) / A C C D / / M P 2 / A C C T$. Note that this scheme was used for all the molecules except $\mathrm{LiCH}_{2}^{-}$in singlet state, and $\mathrm{LiNH}^{-}$in triplet state for which geometries at the mi nimum were found with MP2 using the ACCD basis set. This was done both to compare our methodology to results obtained with $\mathrm{BD}(\mathrm{T}) /$ aug-cc-pVQZ by Tian et al. ${ }^{166}$ and to confirm that $\Delta \mathrm{H}_{\text {acid }}^{298 \mathrm{~K}}\left(\mathrm{CH}_{4}\right)<\Delta \mathrm{H}_{\text {acid }}^{298 \mathrm{~K}}(\mathrm{LiOH})$ indicates that acidities of first-row hydrides can be also correctly assigned. This test was based on a well known dependence: that more electronegative substituents $\left(H_{n} R\right.$, $n=1,2,3,4)$ stabilize the anionic species leading to greater acidity of the neutral molecule. However, exceptions are reported when one of the hydrogen atoms is exchanged with an electropositive al kali metal such as $\mathrm{Li}$ or $\mathrm{Na}^{166}$ For example, $\mathrm{LiCH}_{3}$ is more acidic than $\mathrm{CH}_{4}$, but this is not true for $\mathrm{LiOH}$ which is less acidic than $\mathrm{H}_{2} \mathrm{O}$ molecule, al though oxygen is more electronegative than the carbon atom (see plot presented in Fig. 5.1).

Overall, lithiated first-row hydrides $\left(\mathrm{LiH}_{\mathrm{n}-1} \mathrm{R}\right)$ show opposite behavior in terms of acidityelectronegativity trend when compared to $H_{n} R$ species, viz the more electronegative $R$ the less acidic (or more basic) $\mathrm{LiH}_{\mathrm{n}-1} \mathrm{R}$. The CCSD(T)/ACCD//MP2/ACCT gave virtually the same results for hydrides as $\mathrm{BD}(\mathrm{T}) /$ aug-cc-pVQZ, whereas for their lithiated anal ogs values differ by $5-13 \mathrm{kcal} / \mathrm{mol}$. This is a relatively small loss in the accuracy (1-4\%), and more importantly, the acidity trends are predicted correctly.

The next aspect to test was the level of CC theory necessary to find a proper acidity trend for the first-row hydrides. This was done by carrying out calculations at CCSD level using the same Dunning's basis sets as for the CCSD $(T)$ method. Results are shown at Table 5.5. 


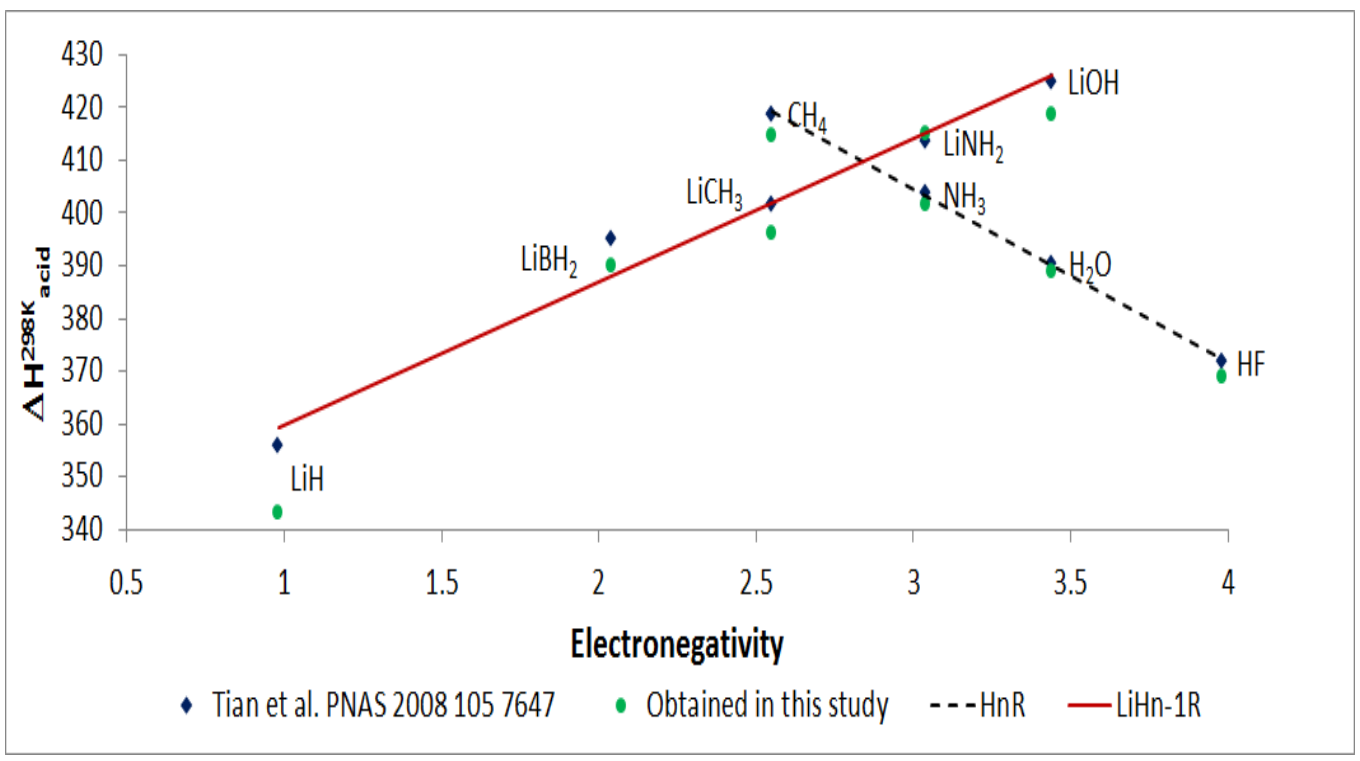

Figure 5.1: Acidity of first-row hydrides $\left(\mathrm{H}_{n} \mathrm{R}\right)$ and their lithiated analogs $\left(\mathrm{LiH}_{\mathrm{n}-1} \mathrm{R}\right)$ vs. electronegativity of $R$ at $298 \mathrm{~K}$. The blue rhombus rectangles represent acidity values obtained by Tian et al. ${ }^{166}$ and the green circles are values calculated in this work. Note that for calculations with $\operatorname{CCSD}(T) / A C C D$ geometry was optimized using MP2/ACCT.

The $\Delta \mathrm{H}_{\text {acid }}(\mathrm{LiOH}) \mathrm{s}$ are lowered by 3.3 or $3.4 \mathrm{kcal} / \mathrm{mol}$ with augmentation of cc-pVDZ or cc-pVTZ basis sets for both CC methods, whereas the decrease of the $\Delta \mathrm{H}_{\text {acid }}\left(\mathrm{CH}_{4}\right)$ for cc-pVDZ/aug-cc-pVDZ at CCSD level is about $1.6 \mathrm{kcal} / \mathrm{mol}$ smaller than for the same basis set pair at $\operatorname{CCSD}(\mathrm{T})$ level. This indicates that singly and doubly excited contributions (also-called singles and doubles) are insufficient to provide the proper trend for acidities for these systems within aug-cc-pVDZ basis set. However, when cc-pVTZ basis set is augmented the $\Delta \mathrm{H}_{\text {acid }}\left(\mathrm{CH}_{4}\right)$ is lowered by almost $12 \mathrm{kcal} / \mathrm{mol}$, which is enough to revers incorrect order $\Delta \mathrm{H}_{\text {acid }}\left(\mathrm{CH}_{4}\right)>\Delta \mathrm{H}_{\text {acid }}(\mathrm{LiOH})$. Moreover, it is encouraging that CCSD/augcc-pVTZ, which is a less demanding in terms of computational cost, can give very similar results to those produces with $\operatorname{CCSD}(T) /$ aug-cc-pVDZ. In summary, in order to obtain the proper acidity trend for $\mathrm{CC}$ methods either triples are necessary when employing aug-cc- 
Table 5.5: Comparison between CCSD and CCSD $(T)$ results in predicting acidities $\left(\Delta \mathrm{H}_{\text {acid }}\right)$ using Dunning's basis sets. Units $\mathrm{kcal} / \mathrm{mol}$ at $\mathrm{OK}$.

\begin{tabular}{|c|c|c|c|c|}
\hline CCSD & cc-pVDZ & aug-cc-pVDZ & cc-pVTZ & aug-cc-pVTZ \\
\hline $\mathrm{LiO}^{-}$ & -51741.2 & -51763.6 & -51791.2 & -51800.1 \\
\hline $\mathrm{LiOH}$ & -52167.8 & -52186.8 & -52222.2 & -52227.9 \\
\hline$\Delta \mathrm{H}_{\text {acid }}$ & 426.6 & 423.2 & 431.0 & 427.7 \\
\hline $\mathrm{CH}_{3}^{-}$ & -24893.0 & -24920.8 & -24931.8 & -24945.6 \\
\hline $\mathrm{CH}_{4}$ & -25341.2 & -25345.9 & -25371.3 & -25372.9 \\
\hline$\Delta \mathrm{H}_{\text {acid }}$ & 448.2 & 425.0 & 439.5 & 427.3 \\
\hline $\operatorname{ccsD}(T)$ & cc-pVDZ & aug-cc-pVDZ & cc-pVTZ & aug-cc-pVTZ \\
\hline $\mathrm{LiO}^{-}$ & -51742.9 & -51766.6 & -51795.4 & -51805.0 \\
\hline $\mathrm{LiOH}$ & -52170.3 & -52190.6 & -52227.8 & -52234.2 \\
\hline$\Delta \mathrm{H}_{\text {acid }}$ & 427.4 & 423.9 & 432.4 & 429.1 \\
\hline $\mathrm{CH}_{3}^{-}$ & -24895.7 & -24925.4 & -24936.8 & -24951.6 \\
\hline $\mathrm{CH}_{4}$ & -25343.6 & -25348.7 & -25374.9 & -25377.0 \\
\hline$\Delta \mathrm{H}_{\text {acid }}$ & 447.9 & 423.3 & 438.1 & 425.4 \\
\hline
\end{tabular}

pVDZ or a bigger basis set is needed at singles and doubles level.

\subsection{Triplet Ground State of $\mathrm{LiO}^{-}$}

Considering this work and the study by Tian and coworkers ${ }^{166}$ the $\mathrm{LiOH}$ has been found to be more basic than $\mathrm{CH}_{4}$. A possible explanation for the unique properties shown by the former compound is the multiplicity (defined as $M=2 S+1$, where $S$ is the total spin of molecule) of lithium monoxide anion $\left(\mathrm{LiO}^{-}\right)$produced by deportation. Mainly the most stable state (i.e., ground state) of $\mathrm{LiO}-$ specie is with $\mathrm{M}=3$ (for which $\mathrm{S}=1$ ), a.k.a. triplet ground-state. The geometrical parameters for $\mathrm{LiO}^{-}$in its lowest energy state are different than when computed as a singlet state (see Fig. 5.2). This might imply that the multiplicity 
has a significant effect on the structure and therefore on the properties of these species.

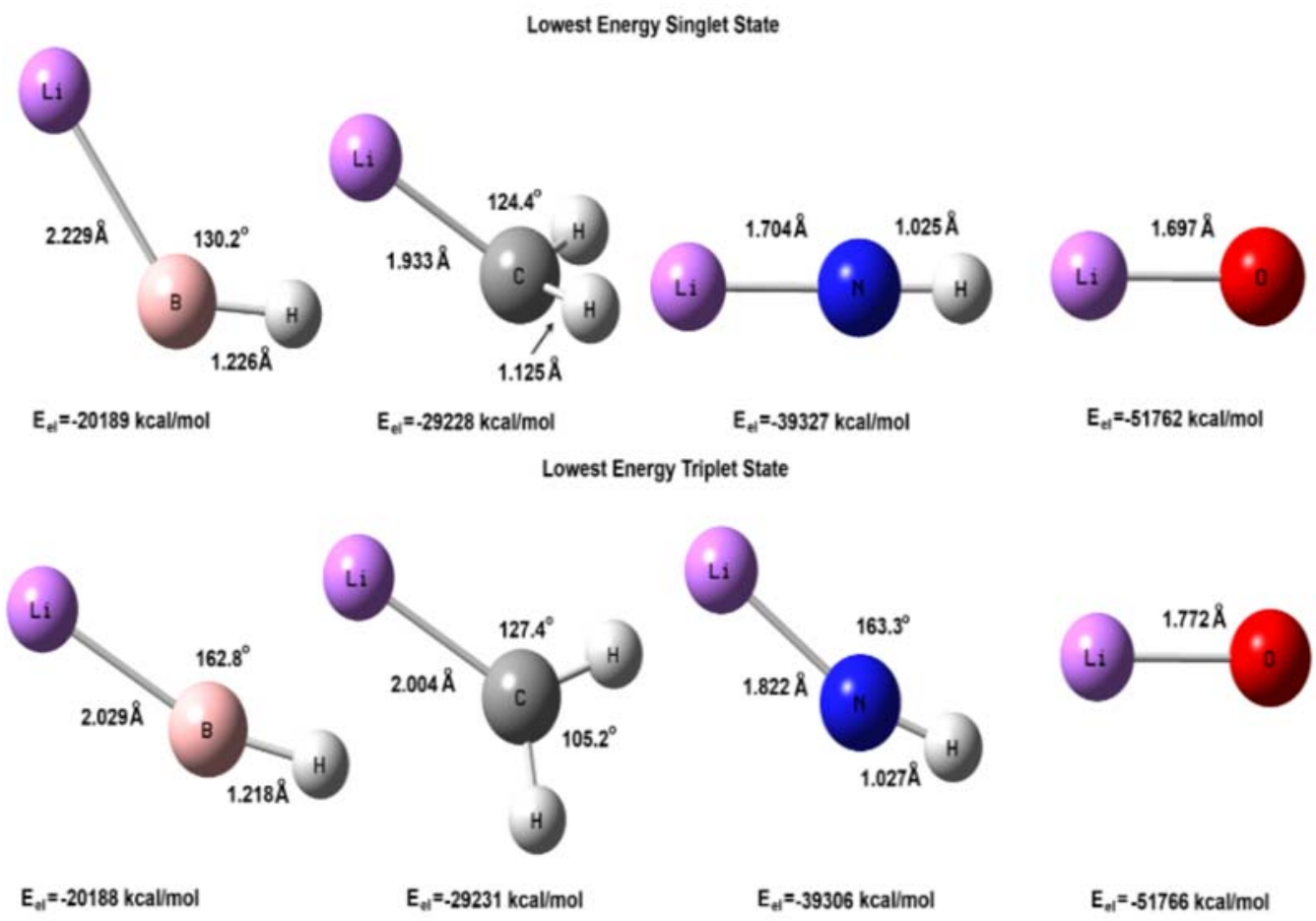

Figure 5.2: Optimized structures of $\mathrm{LiH}_{\mathrm{n}-2} \mathrm{R}^{-}$molecules via MP2/ACCT and Single Point Energies computed with $\operatorname{CCSD}(T) / A C C D$. Purple, pale pink, grey, blue, red and white spheres represent: Li, B, C, N, O and $\mathrm{H}$ atoms, respectively.

Notethat cal culations on $\mathrm{LiCH}_{3}$ with $\mathrm{M}=3$ do not reach the gl obal minima at the MP/ACCT level probably due to the highly mutireference character of this molecule.

Specifically, we showed herein that the Li-O bond of the negative ion in its singlet state $\left({ }^{1} \Sigma^{+}\right.$, with active $\mathrm{MO}$ configuration $\left.\sigma_{2}^{2}, \Pi^{4}, \sigma_{2}^{*}\right)$ is about $0.08 \AA$ shorter than in its triplet state $\left({ }^{3} \Pi\right.$ with active $M O$ configuration $\left.\sigma_{2}^{2}, \Pi^{3}, \sigma_{2}^{\star 1}\right)$. Similarly, the shortening of the bond length has been found for the negative ions of the alkali monoxides in the study of Mintz and coworkers ${ }^{175}$ and it may be caused by a lack of weak bonding between afully occupied $\sigma_{2}$ orbital and an empty $\sigma_{2}^{*}$ orbital, which in triplet state is singly occupied. 
The differences between geometrical structures of single and triplet states of neutral molecules are presented in Fig 5.3). The elongation of Li-O bond al so takes place; however unlike in the case of the anion, the ${ }^{3} \Pi$ state lies above the ${ }^{1} \Sigma^{+}$state.

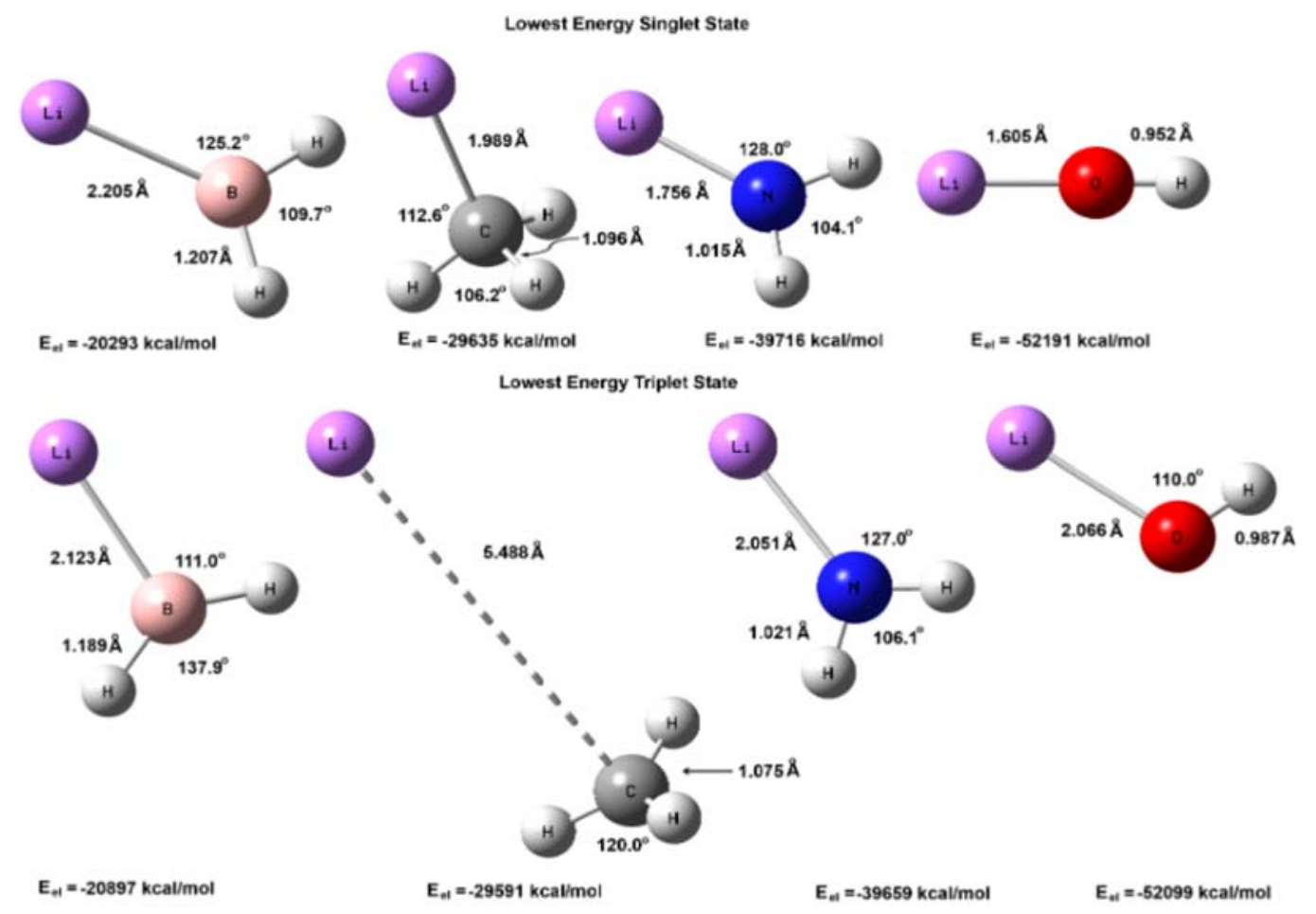

Figure 5.3: Optimized structures of $\mathrm{LiH}_{\mathrm{n}-1} \mathrm{R}$ molecules via MP2/ACCT and Single Point Energies computed with $\operatorname{CCSD}(T) / A C C D$. Purple, pink, grey, blue, red and white spheres represent: $L i, B, C, N, O$ and $H$ atoms, respectively.

At this point of the discussion it is important to stress that the singlet state of $\mathrm{LiO}^{-}$should be energetically above the triplet state. ${ }^{175}$ Hence in al I calculations described so far the multiplicity of this specie was set to $M=3$. Nevertheless, one may ask how well considered here methods would perform if that information was not available. Thus we performed additional test to find if triplet state was indeed predicted as the ground state for $\mathrm{LiO}^{-}$. To our surprise BLYP functional, which performed better than B3LYP (at OK) in finding the 
proper acidity order located the lowest singlet state below the lowest triplet state. This is incorrect and pinpoints the fact that the HF exchange hybridization in the B3LYPfunctional plays a crucial role. In other words without contribution for HF exchange the energy of ${ }^{3} \Pi$ state would be above that of the ${ }^{1} \Sigma^{+}$state. Indeed, cal culations utilizing HF method for the open-shell systems (UHF or ROHF) within aug-cc-pVDZ basis set confirmed that the triplet state is energetically lower than the singlet state. Furthermore, the $\operatorname{CCSD}(T)$ also predicted this ordering.

\subsection{Conclusions}

From results presented in this study it can be concluded that the choice of the basis set is crucial for the correct prediction of the acid-base behavior of the molecules considered herein. Moreover, calculations using chosen "black box" method like $\operatorname{CCSD}(\mathrm{T})$, proved themselves to be equally accurate. Finally, the performance of the popular B3LYP within the augmented double $\zeta$ type basis set (aug-cc-pVDZ or 6-31G**) is inconsistent, i.e., at $0 \mathrm{~K}$ acidities are reported in the incorrect order: $\Delta \mathrm{H}_{\text {acid }}\left(\mathrm{CH}_{4}\right)>\Delta \mathrm{H}_{\text {acid }}(\mathrm{LiOH})$, whereas at $298 \mathrm{~K}$ the situation is reversed. Although ZPE has rather small contribution to the total energy its contribution may change the qual itative picture of theoretical results. This was shown at B3LYP cal culation level. In terms the correctness of predicting the gas phase acidity trend with out ZPE and thermal corrections BLYPis abetter choice between the two DFT functionals. Yet, one has to keep in mind that for compounds with unknown multiplicity of the ground stateBLYP might not be the best choice after all. Since thisfunctional was unable to predict correctly the order of states for $\mathrm{LiO}^{-}$. Summarizing, for virtually all the species considered in this work there was no need to employ sophisticated and costly methods as CAS-AQCC/aug-cc-pVQZ, because the excellent agreement between theory and experiment as obtained in the study by Tian et al. ${ }^{166}$ was determined by the choice of the augmented basis set rather than by the computational method itself. Nonetheless, 
in case of molecules with highly multireference character such as $\mathrm{LiCH}_{3}$ in its triplet state increasing the size of the basis set and/or the level of theory might be necessary. For both the DFT functionals tested herein no evident advantage in employing either one over the $\operatorname{CCSD}(T)$ method was noticed. Advantages coming from the use of DFT are more evident when bigger molecular systems are investigated (for instance, in study presented in Chapter 4). 


\section{Chapter 6}

\section{Concluding Remarks}

In the presented work it has been shown that open-shell molecular species are challenging systems to model even with the state-of-the-art computational methods.

In particular the difficulty of predicting the UV-Vis spectra of $\mathrm{Cu}(\mathrm{II})$ complexes with the 4-imidazole acetate and its ethylated derivative was stressed. Long-Range Corrected functionals were employed for the demanding task of accurate describing both metal- and ligand-based transitions. This scheme is useful in improving the performance of standard hybrid functionals but it has its own limitations; for instance, the range-separation parameter ( $\mathrm{Y}$ ) is system dependent. Hence to truly take advantage of Long-Range Corrected hybrid functionals, the y parameter should be optimized for each given system in order to minimize the delocal ization error, which is related to the incomplete removing of the " many electron SIE". Unfortunately currently the range-separation parameter is optimized in such a way that only the frontier orbitals are involved in the procedure called "tuning from first principles". This leads to the issue of imbal anced treatment of local ized vs. del ocal ized orbitals, which affects predicted UV-Vis spectra. Mainly, it seems that for orbitals of different nature than $\mathrm{HOMO}$ and/or LUMO the procedure al lowing to minimize the delocalization 
error does not work properly. The evidence becomes especial ly convincing when electronic transitions from deeper laying occupied molecular orbitals are considered. Therefore results reported in this work may be helpful in answering questions on how to change the "tuning" procedure in the attempt of avoiding such issues.

Another important aspect in predicting molecular properties of open-shell systems is the proper choice of the basis set. This was illustrated by obtaining the incorrect acidity order for $\mathrm{LiOH}$ vs. $\mathrm{CH}_{4}$ with Dunning's and Pople's basis sets that were not augmented. Although it may seem obvious for a bigger basis set to be a better choice performed calculations can become far too demanding. Hence choosing a basis set that allows to obtain accurate qual itative and quantitative description of a given system with a reasonable request of computational resources may not be as trivial. In case of first-row hydrides and their lithiated analogs, reported in this work, relatively small but augmented basi s sets (augcc-pVDZ and aug-cc-pVTZ) were necessary to achieve the right bal ance between accuracy of the results and computational cost. Furthermore, as long as an aug-cc-pVXZ basis set was utilized the level of theory did not play a crucial rolein obtaining the proper qual itative description. However, such general ization should be taken with caution because open-shell systems may show a strong multireference character (e.g. $\mathrm{LiCH}_{3}$ with $\mathrm{M}=3$ ) which possibly requires higher level of theory than "black box" CCSD(T)/aug-cc-pVTZ. This is an excellent example showing that computational modeling of such species is less intuitive than in case of closed-shell systems.

The educated application of electronic structure theory is a driving force to develop new computational methods. Again this task is more demanding when it refers to open-shell systems. For instance the CR-EOMCC $(2,3)$ approach has been developed to obtain highly accurate excited states and excitation energies. In the presented work, as well as elsewhere, it has been shown that this method is especial ly applicable to describe exited states dominated by double electron excitations. This makes the CR-EOMCC $(2,3)$ very useful for modeling open-shells since doubly excited states need to be considered for an ade- 
quate characterization of the properties. Even though $\operatorname{CR}-\operatorname{EOMCC}(2,3)$ is a high-level ab initio method, robust enough to deal with the multireference character of excited states often characterizing many open-shell systems, the accuracy of cal culated properties suffer from the lack of size-extensivity. Therefore an investigation of the possible sources of the size-extensivity error of this and related Equation-of-Motion Coupled Cluster methods was performed. Elimination of this error is crucial for the application of $\operatorname{CR}-\operatorname{EOMCC}(2,3)$ approach in larger systems, where its value will increase with the size. Based on study discussed in this work it has been concluded that the left (deexcitation) operator constructed for the excited states is the source of the size-extensivity error. This provides new insight that may help to find a better way of designing the deexcitation operator.

The work, as it is, poses the basis for further investigations. In regard to the project addressing the UV-Vis spectra of $\mathrm{Cu}(\mathrm{II})$ complexes with imidazole derivatives the following aspects may be interesting to examine: i) calculations with wave function based methods such as SAC-CISD, EOM-EE-CCSD, EOM-IP-CCSD and EOM-EA-CCSD to validate UV-Vis spectra cal culated with CAM-B3LYP* variants independently from experimental data. This would help to confirm the ability of this level of theory in accurately and reliably predicting $d-d$ and LMCT transitions in open-shell metal-organic complexes such as those considered herein, ii) cal culations with other density based methods (for instance Spin-Flip TDDFT) to address double excitations that are likely to be responsible for some of the predicted absorption peaks, iii) modeling of solvent environment to predict solvatochromic effects in the UV-Vis spectra of the $\left[\mathrm{Cu}(\mathrm{IAC})_{2}\right]$ with focus on the role of the range-separation parameter and the amount of exact exchange.

In regard to the project inquiring the possible sources of the size-extensive error in CREOMCC methods the next step could be constructing the deexcitation operator for excited state to be equival ent to the ground state. This may be the remedy for developing an accurate and size-extensive method for studying excited states of both cl osed- and open-shell species. 
Lastly it could be useful to see if basis set larger than aug-cc-pVTZ employed without increasing the level of theory will be able to properly describe $\mathrm{LiCH}_{3}$ in its triplet state or if, on the contrary, the single reference methods such as $\operatorname{CCSD}(T)$ are not appropriate for this task. 


\section{References}

${ }^{1}$ W. Heinsenberg, Z. Phys., 33, 879, (1925).

2 E. Schrödinger, Ann. Phys., 79, 361, (1926).

${ }^{3}$ E. Hückel, Z. Phys., 70, 204, (1931).

${ }^{4}$ C. J. Cramer, Essentials of Computational Chemistry: Theories and Models, (John Wiley \& Sons, Ltd., England, 2004), 2nd Ed., pp. 203-243; 247-299.

${ }^{5}$ A. Szabó, and N. S. Ostlund, Modern Quantum Chemistry Introduction to Advanced Electronic Structure Theory, (Dover Publications, Inc., Mineola, NY, 1996).

${ }^{6}$ F. Jensen, Introduction to Computational Chemistry, (John Wiley \& Sons Ltd, West Sussex, England, 1999), pp. 53-80; 98-149.

7 J. S. Andrews, D. Jayatilake, R. G. A. Bone, N. C. Handy, and R. D. Amos, Chem. Phys. Lett., 183, 423, (1991).

${ }^{8}$ C. Möller, and M. S. Plesset, Phys. Rev., 46, 618, (1934).

${ }^{9}$ M. L. Leininger, W. D. Allen, H. F. Schaefer III, and C. D. Sherrill, J. Chem. Phys., 
112, 9213, (2000).

${ }^{10}$ W. Kutzelnigg, and W. Klopper, J. Chem. Phys., 94, 1985, (1991).

${ }^{11}$ D. P. Tew, and W. Klopper, J. Chem. Phys., 123, 074101, (2005).

${ }^{12}$ I. N. Levine,Quantum Chemistry, 6th Ed., (Pearson Education, Inc., Upper Saddle River, NJ, 2009), pp. 440-450; 566-618.

${ }^{13}$ H. Partridge, J. Chem. Phys., 90, 1043, (1989).

${ }^{14}$ S. R. Langoff, and E. R. Davidson, Int. J. Quantum Chem., 8, 61, (1974).

${ }^{15}$ A. I. Krylov, Chem. Phys. Lett., 350, 522, (2001)

${ }^{16}$ A. I. Krylov, and C. D. Sherrill, J. Chem. Phys., 116, 3194, (2002).

${ }^{17}$ F. Coester, Nucl. Phys., 7, 421, (1958).

${ }^{18}$ F. Coester, and H. Kümmel, Nucl. Phys., 17, 477, (1960).

19 J. Čížek, J. Chem. Phys., 45, 4256, (1966).

20 J. Čižek, Adv. Chem. Phys., 14, 35, (1969).

${ }^{21}$ J. Paldus, J. Čížek, and I. Shavitt, Phys. Rev. A, 5, 50, (1972).

${ }^{22}$ R. J. Bartlett, and G. D. Purvis III, Int. J. Quantum Chem., 14, 561, (1978).

${ }^{23}$ R. F. Bishop, and K. H. Lührmann, Phys. Rev. B, 17, 3757, (1978) 
${ }^{24}$ R. J. Bartlett, and G. D. Purvis III, Phys. Scr., 21, 225, (1980).

${ }^{25}$ G. D. Purvis III, and R. J. Bartlett, J. Chem. Phys., 76, 1910, (1982).

26 J. Noga and R. J. Bartlett, J. Chem. Phys., 86, 7041, (1987).

27 J. Noga and R. J. Bartlett, J. Chem. Phys., 89, 3401, (1988) (Erratum).

${ }^{28}$ K. Raghavachari, G. W. Trucks, J. A. Pople, and M. Head-Gordon, Chem. Phys. Lett., 157, 479, (1989).

${ }^{29}$ M. Urban, J. Noga, S. J. Cole, and R. J. Bartlett, J. Chem. Phys., 83, 4041, (1985).

${ }^{30}$ R. J. Bartlett, and M. Musial, Rev. Mod. Phys., 79, 291, (2007).

${ }^{31}$ K. Emrich, Nucl. Phys. A, 351, 379, (1981).

${ }^{32}$ K. Emrich, Nucl. Phys. A, 351, 397, (1981).

${ }^{33}$ D. C. Comeau, and R. J. Bartlett, Chem. Phys. Lett., 207, 414, (1993).

${ }^{34}$ P. Čársky, J. Paldus, and J. Pittner (Eds.), Recent Progress in Coupled Cluster Methods: Theory and Applications, (Springer Science, Berlin, 2010), Vol. 11, pp. 455-489.

35 J. Noga, R. J. Bartlett, and M. Urban, Chem. Phys. Lett., 134, 126, (1987).

${ }^{36}$ Y. J. Bomble, J. F. Stanton, M. Kállay, and J. Gauss, J. Chem. Phys., 123, 054101, (2005).

${ }^{37}$ W. Kohn, and L. J. Sham, Phys. Rev., 140, A1133, (1965). 
${ }^{38}$ P. O. Löwdin, Phys. Rev., 97, 1474, (1955).

${ }^{39}$ K. A. Brueckner, Phys. Rev., 97, 1353, (1955).

${ }^{40}$ K. Jankowski, and K. Rubinic, Mol. Phys., 100, 1741,(2002).

${ }^{41}$ G. E. Scuseria, and V. N. Staroverov, Theory and Applications of Computational Chemistry: The First Forty Years, C. Dykstra, G. Frenking, K. S. Kim, G. E. Scuseria (Eds.), (Elsevier, Amsterdam 2005), pp. 669-724.

${ }^{42}$ A. J. Cohen, P. Mori-Sánchez, and W. Yang, Chem. Rev., 112, 289, (2012).

${ }^{43}$ R. Peverati, and D. G. Truhlar, in press, arXiv:1212.0944.

${ }^{44}$ W. Kohn, and L. J. Sham, Phys. Rev, B136, 864, (1964).

${ }^{45}$ S. F. Sousa, P. A. Fernandes, and M. João Ramos, J. Phys. Chem. A, 111, 10439, (2007)

${ }^{46}$ N. Argaman, G. Makov, Am. J. Phys. 68, 69, (2000).

${ }^{47}$ D. S. Sholl, and J. A. Steckel, Density Functional Theory: A practical intoduction, (J. Wiley \& Sons, Inc., New Jersey, 2009), pp. 10-16.

${ }^{48}$ W. Kohn, and L. J. Sham, Phys. Rev., 140, A1133, (1965).

${ }^{49}$ A. R. Leach, Molecular Modeling Principles and Aplications, (Prentice Hall, England 2001) 2nd Ed., pp. 126-138.

${ }^{50}$ S. H. Vosko, L. Wilk, M. Nusair, Can. J. Phys., 58, 1200, (1980). 
${ }^{51}$ A. D. Becke, Phys. Rev. A, 39, 3761, (1988).

52 J. P. Perdew, J. A. Chevary, S. H. Vosko, K. A. Jackson, M. R. Pederson, D. J. Singh, and C. Fiol hais, Phys. Rev. B, 46, 6671, (1992)

53 J. M. Tao, J. P. Perdew, V. N. Staroverov, and G. E. Scuseria, Phys. Rev. Lett., 91, 146401, (2003).

${ }^{54}$ A. D. Becke, J. Chem. Phys., 98, 5648, (1993).

55 J. P. Perdew, J. A. Chevary, S. H. Vosko, K. A. Jackson, M. R. Pederson, D. J. Singh, and C. Fiol hais, Phys. Rev. B, 48, 497 (1993).

${ }^{56}$ C. Le, W. Yang, and R. G. Parr, Phys. Rev. B, 37, 785, (1988).

57 J. P. Perdew, and Y. Wang, Phys. Rev. B., 45, 13244, (1992).

${ }^{58}$ P. J. Stephens, F. J. Devlin, C. F. Chabalowski, and M. J. Frisch, J. Phys. Chem., 98, 11623, (1994).

${ }^{59}$ S. Grimme, L. Goerigk, and R. F. Fink, MREs, Comput. Mol. Sci., 2, 886, (2012).

${ }^{60}$ A. Dreuw, and M. Head-Gordon Chem. Rev., 105, 4009, (2005).

${ }^{61}$ S. R. Gwal tney, and M. Head-Gordon, Chem. Phys. Lett., 323, 21, (2000).

62 S. Hirata, P. -D. Fan, A. A. Auer, M. Nooijen, and P. Piecuch, J. Chem. Phys., 121, 12197, (2004).

${ }^{63}$ K. Kowal ski, and P. Piecuch, J. Chem. Phys, 113, 18, (2000). 
${ }^{64}$ K. Kowalski, and P. Piecuch, J. Chem. Phys, 113, 5644, (2000).

${ }^{65}$ P. Piecuch, K. Kowal ski, and I. S. O. Pimienta, Int. J. Mol. Sci., 3, 475, (2002).

${ }^{66}$ P. Piecuch, K. Kowal ski, I. S. O. Pimienta, P.-D. Fan, M. Lodriguito, M. J. McGuire, S. A. Kucharski, T. Kuś, and M. Musial, Theor. Chem. Acc.,112, 349, (2004).

${ }^{67}$ P. Piecuch, and M. Włoch, J. Chem. Phys., 123, 224105, (2005).

${ }^{68}$ K. Kowalski, and P. Piecuch, Chem. Phys. Lett., 344, 165, (2001).

${ }^{69}$ M. J. McGuire, and P. Piecuch, J. Am. Chem. Soc., 127, 2608, (2005).

${ }^{70}$ M. Wloch, J. R. Gour, K. Kowalski and P. Piecuch, J. Chem. Phys, 122, 214107, (2005).

${ }^{71}$ P. Piecuch, M. Włoch, J. R. Gour, and A. Kinal, Chem. Phys. Lett., 418, 467, (2006).

${ }^{72}$ E. A. Salter, G. W. Trucks, and R. J. Bartlett, J. Chem. Phys., 90, 1752, (1989).

${ }^{73}$ M. Włoch, J. R. Gour, and P. Piecuch, J. Phys. Chem. A,111, 11359, (2007).

${ }^{74}$ D. J. Rowe, Rev. Mod. Phys., 40, 153, (1968).

${ }^{75}$ T. H. Jr. Dunning, and V. McKoy, J. Chem. Phys., 47, 1735, (1967).

${ }^{76}$ J. Simons, and W. D. Smith, J. Chem. Phys., 58, 4899, (1973).

77 J. Geertsen, M. Rittby, and R. J. Bartlett, Chem. Phys. Lett., 164, 57, (1989).

${ }^{78}$ H. Sekino, and R. J. Bartlett, Adv. Quantum Chem., 35, 149, (1999). 
${ }^{79}$ K. Kowalski, and P. Piecuch, J. Chem. Phys., 115, 2966, (2001).

${ }^{80}$ K. Kowalski, and P. Piecuch, J. Chem. Phys., 116, 7411, (2002).

${ }^{81}$ P. Piecuch, K. Kowalski, I. S. O. Pimienta, and M. J. McGuire, Int. Rev. Phys. Chem., 21, 527, (2002).

${ }^{82}$ P. Piecuch, J. R. Gour, and M. Włoch, Int. J. Quantum Chem., 109, 3268, (2009).

${ }^{83}$ M. Włoch, M. D., Lodriguito, P. Piecuch, and J. R. Gour, Mol. Phys., 104, 2149, (2006).

${ }^{84}$ K. Kowalski, and P. Piecuch, J. Chem. Phys., 120, 1715, (2004).

${ }^{85}$ H. J. Monkhorst, Int. J. Quantum Chem. Quantum Chem. Symp., 11, 421, (1977).

${ }^{86}$ E. Dal gaard, and H. J. Monkhorst, Phys. Rev. A, 28, 1217, (1983).

${ }^{87}$ D. Mukherjee, and P. K. Mukherjee, Chem. Phys., 39, 325, (1979).

${ }^{88}$ M. Ehara, and H. Nakatsuji, Recent Progress in Coupled Cluster Methods: Theory and Applications, P. Č́rsky et al. (Eds.), pp. 79112.

${ }^{89}$ H. Nakatsuji, Chem. Phys. Lett., 177, 331, (1991).

${ }^{90}$ M. Ehara, and H. Nakatsuji, Chem. Phys. Lett., 282, 347, (1998).

${ }^{91}$ A. I. Krylov, Chem. Phys. Lett., 338, 375, (2001).

${ }^{92}$ L. V. Slipchenko, and A. I. Krylov, J. Chem. Phys., 117, 4694, (2002).

${ }^{93}$ P. U. Manohar, and A. I. Krylov, J. Chem. Phys., 129, 194105, (2008). 
${ }^{94}$ Y. Zhao, D. G. Truhlar, Theor. Chem. Acc., 120, 215, (2008).

${ }^{95}$ D. C. Langreth, and J. P. Perdew, Phys. Rev. B, 15, 2884, (1977).

${ }^{96}$ A. Savin, F. Colonna, and R. Pollet, Int. J. Quantum Chem., 93, 166, (2003).

${ }^{97}$ S. Grimme, and F. Neese, J. Chem. Phys., 127, 154116, (2007).

${ }^{98}$ F. Furche, and T. Van Voorhis, J. Chem. Phys., 122, 164106, (2005).

${ }^{99}$ A. W. Lange, M. A. Rohrdanz, and J. M. Herbert, J. Phys. Chem. B, 112, 6304 (2008).

${ }^{100}$ D. J. Tozer, J. Chem. Phys., 119, 12697 (2003).

${ }^{101}$ R. D. Adamson, J. P. Dombroski, and P. M. W. Gill, Chem. Phys. Lett., 254, 329, (1996).

102 T. Leininger, H. Stoll, H-J. Werner, and A. Savin, Chem. Phys. Lett., 275, 151, (1997).

${ }^{103}$ T. Yanai, D. P. Tew, and N. C. Handy, Chem. Phys. Lett., 393, 51, (2004).

${ }^{104}$ M. A. Rohrdanz, K. M. Martins, and J. M. Herbert, J. Chem. Phys., 130, 054112, (2009).

${ }^{105}$ Y. Zhang, and W. Yang, Theor. Chem. Acc., 103, 346, (2000).

106 O. A. Vydrov, and G. E. Scuseria, J. Chem. Phys., 126, 154109 (2007).

107 J. P. Perdew, and A. Zunger, Phys. Rev. B, 23, 5048, (1981).

${ }^{108}$ A. J. Cohen, P. Mori-Sánchez, W. Yang, Science, 321, 792, (2008).

109 J. P. Perdew, R. G. Parr, M. Levy, and J. L. Bal duz Jr., Phys. Rev. Lett., 49, 1691, (1982). 
110 J.-D. Chai, and P.-T. Chen, Phys. Rev. Lett., 110, 033002, (2013).

${ }^{111}$ M. Srebro, and J. Autschbach, J. Phys. Chem. Lett., 3, 576, (2012).

${ }^{112}$ T. Körzdörfer, R. M. Parrish, N. Marom, J. S. Sears, C. D. Sherrill, and J-L. Brédas, Phys. Rev. B., 86, 205110, (2012).

${ }^{113}$ N. Bauman, privte communication.

${ }^{114}$ C. E. Dykstra, G. Frenking, K. S. Kim, G. E. Scuseria (Eds.), Theory and Applications of Computational Chemistry: The First Forty Years, (Elsevier, Amsterdam, 2005), pp. 472-476.

${ }^{115}$ T. Shiozaki, K. Hirao, S. Hirata, J. Chem. Phys., 126, 244106, (2007).

${ }^{116}$ O. Christiansen, H. Koch, P. Jørgensen, J. Olsen, Chem. Phys. Lett., 256, 185, (1996).

${ }^{117}$ Basis sets were obtained from the Extensible Computational Chemistry Environment Basis Set Database, Version 02/25/04, as developed and distributed by the Molecular Science Computing Facility, Environmental and Molecular Sciences Laboratory which is part of the Pacific Northwest Laboratory, P. O. Box 999, Richland, Washington 99352, USA, and funded by the US Department of Energy.

${ }^{118}$ M. W. Schmidt, K. K. Baldridge, J. A. Boatz, S. T. Elbert,M. S. Gordon, J. H. Jensen, S. Koseki, N. Matsunaga, K. A. Nguyen, S. J. Su, T. L. Windus, M. Dupuis, J. A. Montgomery,J. Comput. Chem., 14, 1347, (1993).

${ }^{119}$ M. S. Gordon, M. W. Schmidt, Theory and Applications of Computational Chemistry: The First Forty Years, C. E. Dykstra, G. Frenking, K. S. Kim, G. E. Scuseria (Eds.), (Elsevier, Amsterdam, 2005), pp. 1167-1189. 
${ }^{120}$ A. Banerje, J. Li, J. B. Fuggit, D. J. Averill, N. Tyminska, W. W. Brennessel, M. D. Sevilla, and F. A. Chavez, Inorg. Chim. Acta, in press, doi: 10.1016/j.ica.2013.06.020., (2013).

${ }^{121}$ N. Tyminska, J. V. Koppen, and M. M. Szczęśniak, "Assessment of the nonempirically tuned CAM-B3LYP* in predicting UV-Vis spectrum of the $\mathrm{Cu}(\mathrm{II})$ complex with imidazole-4 acetate", in preparation.

${ }^{122}$ C. Madsen, A. A. Jensen, T. Liljefors, U. Kristiansen, B. Nielsen, C. P. Hansen, M. Larsen, B. Ebert, B. Bang-Andersen, P. Krogsgaard-Larsen, and B. Frølund, J. Med. Chem., 50, 4147, (2007).

${ }^{123}$ P. Drożdżewski, B. Pawlak, and T. Głowiak, Polish J. Chem., 75, 1711, (2001).

${ }^{124}$ P. Drożdżewski, B. Pawlak, and T. Głowiak, J. Coord. Chem., 55, 735, (2002).

${ }^{125}$ P. Drożdżewski, and B. Pawlak, Specrochim. Acta A, 60, 1527, (2004).

${ }^{126}$ P. Romaniello, D. Sangalli, J. A. Berger, F. Sottile, L. G. Molinari, L. Reining, and G. Onida, J. Chem. Phys., 130, 044108, (2009).

${ }^{127}$ X. Peng, G.-H. Cui, D.-J. Li, S.-Z. Wu, and Y.-M Yu, J. Mol. Struct., 971, 47, (2010).

${ }^{128}$ S. Sinnecker, and F. Neese, J. Comput. Chem., 27, 1463, (2006).

${ }^{129}$ M. Atanasov, P. Comba, B. Martin, V. Müller, G. Rajaraman, H. Rohwer, and S. Wunderlich, J. Comp. Chem., 27, 1263, (2006).

130 G. F. Caramori, R. L. T. Parreira, and M. Da Costa Ferreira, Int. J. Quantum Chem., 112, 625, (2012). 
${ }^{131}$ F. Wang, and T. Ziegler, Mol. Phys., 102, 2585, (2004).

${ }^{132}$ G. Wu, H. Stewart, F. D. Lemon, H. Cox, and A. J. Stace, Mol. Phys., 108, 1199, (2010).

${ }^{133}$ P. M. W. Gill, R. D. Adamson, and J. A. Pople, Mol. Phys., 88, 1005, (1996).

${ }^{134}$ S. Grimme, J. Chem. Phys., 118, 9095, (2003).

${ }^{135}$ F.-Q. Bai, J. Wang, B.-H. Xia, Q.-J. Pan, and H.-X. Zhang, Dalton Trans., 41, 8441, (2012).

${ }^{136}$ D. M. Chipman, J. Chem. Phys., 112, 5558, (2000).

${ }^{137}$ M. E. Casida, Recent Advances in Density Functional Methods, Part 1, D. P. Chong (Eds.), (World Scientific, Singapore, 1995), pp. 155-192.

138 J. D. Chai , and M. Head-Gordon, J. Chem. Phys., 128, 084106, (2008).

139 T. M. Henderson, B. G. Janesko, and G. E. Scuseria, J. Chem. Phys., 128, 194105, (2008)

${ }^{140}$ M. Ernzerhof, and J. P. Perdew, J. Chem. Phys, 109, 3313, (1998).

${ }^{141}$ D. Jacquemin, V. Wathelet, E. A. Perpète, and C. Adamo, J. Chem. Theory Comput., 5, 2420, (2009).

142 Gaussian 03, Revision E.01, M. J. Frisch, G. W. Trucks, H. B. Schlegel, G. E. Scuseria, M. A. Robb, J. R. Cheeseman, J. A. Montgomery, Jr., T. Vreven, K. N. Kudin, J. C. Burant, J. M. Millam, S. S. Iyengar, J. Tomasi,V. Barone, B. Mennucci, M. Cossi, G. Scalmani, N. Rega, G. A. Petersson, H. Nakatsuji, M. Hada, M. Ehara, K. Toyota, 
R. Fukuda, J. Hasegawa, M. Ishida, T. Nakajima, Y. Honda, O. Kitao, H. Nakai, M. Klene, X. Li, J. E. Knox, H. P. Hratchian, J. B. Cross, C. Adamo, J. Jaramillo, R. Gomperts, R. E. Stratmann, O. Yazyev, A. J. Austin, R. Cammi, C. Pomelli, J. W. Ochterski, P. Y. Ayala, K. Morokuma, G. A. Voth, P. Salvador, J. J. Dannenberg, V. G. Zakrzewski, S. Dapprich, A. D. Daniels, M. C. Strain, O. Farkas, D. K. Malick, A. D. Rabuck, K. Raghavachari, J. B. Foresman, J. V. Ortiz, Q. Cui, A. G. Baboul, S. Clifford, J. Cioslowski, B. B. Stefanov, G. Liu, A. Liashenko, P. Piskorz, I. Komaromi, R. L. Martin, D. J. Fox, T. Keith, M. A. Al-Laham, C. Y. Peng, A. Nanayakkara, M. Chall lacombe, P. M. W. Gill, B. Johnson, W. Chen, M. W. Wong, C. Gonzal ez, and J. A. Pople, Gaussian, Inc., Wall lingford CT, (2004).

${ }^{143}$ GAUSSIAN 09, Revision B.01, M. J. Frisch, G. W. Trucks, H. B. Schlegel, G. E. Scuseria, M. A. Robb, J. R. Cheeseman, G. Scal mani, V. Barone, B. Mennucci, G. A. Petersson, H. Nakatsuji, M. Caricato, X. Li, H. P. Hratchian, A. F. Izmaylov, J. Bloino, G. Zheng, J. L. Sonnenberg, M. Hada, M. Ehara, K. Toyota, R. Fukuda, J. Hasegawa, M. Ishida, T. Nakajima, Y. Honda, O. Kitao, H. Nakai, T. Vreven, J. A. Montgomery, J., J. E. Peralta, F. Ogliaro, M. Bearpark, J. J. Heyd, E. Brothers, K. N. Kudin, V. N. Staroverov, T. Keith, R. Kobayashi, J. Normand, K. Raghavachari, A. Rendell, J. C. Burant, S. S. Iyengar, J. Tomasi, M. Cossi, N. Rega, J. M. Millam, M. Klene, J. E. Knox, J. B. Cross, V. Bakken, C. Adamo, J. Jaramillo, R. Gomperts, R. E. Stratmann, O. Yazyev, A. J. Austin, R. Cammi, C. Pomelli, J. W. Ochterski, R. L. Martin, K. Morokuma, V. G. Zakrzewski, G. A. Voth, P. Salvador, J. J. Dannenberg, S. Dapprich, A. D. Daniels, O. Farkas, J. B. Foresman, J. V. Ortiz, J. Cioslowski, and D. J. Fox, Gaussian, Inc., Wall lingford CT, 2010.

${ }^{144}$ M. Schreiber, M. R. Silva-Junior, S. P. A. Sauer, and W. Thiel, J. Chem. Phys., 128, 134110, (2008).

${ }^{145}$ A. Ipatov, F. Cordova, L. J. Doriol and M. E. Casida, J. Mol. Struct.: THEOCHEM, 
914, 60, (2009).

${ }^{146}$ M. E. Casida, and D. R. Sal ahub, J. Chem. Phys., 113, 8918, (2000).

${ }^{147}$ Y. Tawada, T. Tsuneda, S. Yanagisawa, T. Yanai, and K. Hirao, J. Chem. Phys., 120, 8425, (2004).

${ }^{148}$ R. K. Szilagyi, M. Metz, and E. I. Solomon, J. Phys. Chem. A, 106, 2994, (2002).

${ }^{149}$ R. Janes, and E. Moore Metal-Ligand Bonding, E. W. Abel (Eds.), (The Open University, 2004), pp. 57-65.

${ }^{150}$ R. Baer, E. Livshits, and U. Salzner, Annu. Rev. Phys. Chem., 61, 85, (2010).

151 J. F. Janak, Phys. Rev. B, 18, 7165, (1978).

${ }^{152}$ T. Stein, L. Kronik, and R. Baer, J. Chem. Phys., 131,244119, (2009).

${ }^{153}$ T. Stein, H. Eisenberg, L. Kronik, and R. Baer, Phys. Rev. Lett., 105, 266802, (2010).

${ }^{154}$ A. B. P. Lever, Inorganic electronic spectroscopy, Studies in Physical and Theoretical Chemistry Series 33, (Elsevier, New York, 1984), 2nd Ed., pp. 680-710.

${ }^{155}$ M. A. Rohrdanz, and J. M. Herbert, J. Chem. Phys. 129, 034107, (2008).

${ }^{156}$ L. B. A. Oliveira, T. L. Fonseca, K. Coutinho, and S. Canuto, Chem. Phys. Lett. 514, 251 (2011).

${ }^{157}$ L. Goergik, and S. Grimme, J. Chem. Phys. 132, 184103, (2010).

${ }^{158}$ M. Cossi, and V. Barone, J. Chem. Phys. 112, 2427, (2000). 
${ }^{159}$ D. Maurice, and M. Head-Gordon, J. Phys. Chem., 100, 6131, (1996).

${ }^{160}$ D. Feller, J. Comp. Chem., 17, 1571, (1996).

${ }^{161}$ K. L. Schuchardt, B.T. Didier, T. Elsethagen, L. Sun, V. Gurumoorthi, J. Chase, J. Li, and T.L. Windus, J. Chem. Inf. Model., 47, 1045, (2007).

${ }^{162}$ T. H. Dunning, Jr., J. Chem. Phys., 90, 1007 (1989).

163 W. J. Hehre, R. Ditchfield and J. A. Pople, J. Chem. Phys., 56, 2257 (1972)

164 J. D. Dill and J. A. Pople, J. Chem. Phys., 62, 2921 (1975).

${ }^{165} \mathrm{~N}$. Tyminska, M. Wloch and T. Royappa, "Importance of basis set in predicting gasphase acidity: A study of the first-row hydrides and their lithiated analogs", to be submitted to Int. J. Quantum Chem., (2013).

${ }^{166}$ Z. Tian, B. Chan, M. B. Sullivan, L. Random, and S. R. Kass, PNAS, 105, 7647, (2008).

${ }^{167}$ C. E. Dykstra, Chem. Phys. Lett., 45, 466, (1977).

${ }^{168}$ N. C. Handy, J. A. Pople, M. Head-Gordon, K. Raghavachari, and G. W. Trucks, Chem. Phys. Lett, 164, 185, (1989).

${ }^{169}$ P. G. Szaley, and R. J. Bartlett, J. Chem. Phys., 103, 3600, (1995).

170 P. G. Szalay, and R. J. Bartlett, Chem. Phys. Lett., 214, 481, (1993).

${ }^{171}$ M. W. Wong, and L. Random, J. Phys. Chem., 99, 8582, (1995). 
${ }^{172}$ E. J. Bylaska, W. A. de Jong, N. Govind, K. Kowalski, T. P. Straatsma, M. Valiev, H. J. J. van Dam, D. Wang, E. Apra, T. L. Windus, J. Hammond, J. Autschbach, P. Nichols, S. Hirata, M. T. Hackler, Y. Zhao, P.-D. Fan, R. J. Harrison, M. Dupuis, D. M. A. Smith, K. Glaesemann, J. Nieplocha, V. Tipparaju, M. Krishnan, A. VazquezMayagoitia, L. Jensen, M. Swart, Q. Wu, T. Van Voorhis, A. A. Auer, M. Nooijen, L. D. Crosby, E. Brown, G. Cisneros, G. I. Fann, H. Fruchtl, J. Garza, K. Hirao, R. Kendall, J. A. Nichols, K. Tsemekhman, K. Wolinski, J. Anchell, D. Bernholdt, P. Borowski, T. Clark, D. Clerc, H. Dachsel, M. Deegan, K. Dyall, D. Elwood, E. Glendening, M. Gutowski, A. Hess, J. Jaffe, B. Johnson, J. Ju, R. Kobayashi, R. Kutteh, Z. Lin, R. Littlefield, X. Long, B. Meng, T. Nakajima, S. Niu, L. Pollack, M. Rosing, G. Sandrone, M. Stave, H. Taylor, G. Thomas, J. H. van Lenthe, A. Wong, Z. Zhang "NWChem, A Computational Chemistry Package for Paral lel Computers, Version 4.6" (2004), Pacific Northwest National Laboratory, Richland, Washington 99352-0999, USA.

${ }^{173}$ R. A. Kendall, E. Aprà, D. E. Bernholdt, E. J. Bylaska, M. Dupuis, G. I. Fann, R. J. Harrison, J. Ju, J. A. Nichols, J. Nieplocha, T. P. Straatsma, T. L. Windus, A. T. Wong, Computer Phys. Comm., 128, 260, (2000).

${ }^{174}$ E. Papajak, and D. G. Truhlar, J. Chem. Theory Comput., 6, 597, (2010).

${ }^{175}$ B. Mintz, B. Chan, M. B. Sullivan, T. Buesgen, A. P. Scott, S. R. Kass, L. Random, and A. K. Wilson, J. Phys. Chem. A, 113, 9501, (2009). 